Participatory Policy Analysis for Climate Change Adaptation

From Individual Perceptions to Collective Behaviour 



\title{
PARTICIPATORY POLICY ANALYSIS FOR CLIMATE CHANGE ADAPTATION
}

\author{
FROM INDIVIDUAL PERCEPTIONS TO COLLECTIVE \\ BEHAVIOUR
}

DISSERTATION

to obtain

the degree of doctor at the University of Twente, on the authority of the rector magnificus, prof.dr. T.T.M. Palstra,

on account of the decision of the Doctorate Board, to be publicly defended

on Wednesday 13 February 2019 at 14.45 hrs

by

Sara Mehryar

born on 21 March 1985

in Rasht, Iran 
This thesis has been approved by

Prof.dr.ir. M.F.A.M. van Maarseveen, supervisor

Prof.dr. R.V. Sliuzas, supervisor

ITC dissertation number 343

ITC, P.O. Box 217, 7500 AE Enschede, The Netherlands

ISBN $978-90-365-4726-0$

DOI $10.3990 / 1.9789036547260$

http://doi.org/10.3990/1.9789036547260

Cover designed by Vahid Yazdanpanah and Benno Masselink Painting 'The Harvesters' (1565) by Pieter Bruegel the Elder Printed by ITC Printing Department Copyright (c) 2019 by Sara Mehryar

U GACULTY OF GEO-INFORMATION SCIENCE AND EARTH OBSERVATION 
Graduation committee:

Chairman/secretary

Prof.dr.ir. A. Veldkamp University of Twente

\section{Supervisors}

Prof.dr.ir. M.F.A.M. van Maarseveen University of Twente

Prof.dr. R.V. Sliuzas

University of Twente

\section{Members}

Prof.dr. K. Pfeffer

University of Twente

Dr. D. Reckien

University of Twente

Prof.dr.ir.G.J. Hofstede

Wageningen University

Prof.dr. T. Schroder

University of Potsdam

Dr. N. Schwarz

University of Twente 
To my lovely parents for their never ending

Support. Orust and QLave 


\section{Acknowledgements}

Doing this PhD would not have been possible without the help and support of so many special people. I would like to express my deepest gratitude to some of them who helped me the most during the last years.

First and foremost, I would like to thank my promoter Prof. dr. ir. Martin van Maarseveen for giving me the opportunity of doing this research in ITC, the freedom to find my own research path and his prompt guidance and supports along the way. Thank you for your invaluable scientific contribution to many ideas of this thesis, and the confidence that you placed in my work over the five years of my PhD. It has been an incredible honour and privilege to have you as my promoter, and for that, I am forever thankful.

Secondly, I express my gratitude to my supervisor (co-promoter) Prof. dr. Richard Sliuzas for his kind support and guidance, especially, at the times that I was stuck in my research. This thesis would not be possible without your insightful guidance, friendly advices, and critical comments. The lessons that I learned from your scientific vision and working manners will undoubtedly be invaluable in my future personal and professional life.

I would also like to acknowledge the invaluable role of Ali Sharifi, Diana Reckien, and Nina Schwarz in writing the chapters of this thesis. I have benefited from the unconditional support, constructive feedback and kind encouragements of Dr. Sharifi from the beginning of my PhD. I was fortunate to meet Diana Reckien in 2015 in ITC, start working with her on developing FCM models (which became a crucial part of this thesis) and benefit from all her tender guidance and insightful feedbacks on my research. I also had the great chance of meeting Nina Schwarz and start working with her on developing $A B M$ in the last year of my PhD. Many thanks, Nina, for always being available for my questions, being extremely helpful in solving the problems and challenges that I faced in developing my model, and, of course, revising my last minute papers!

I extend my deep appreciation to the 60 farmers and 40 policy makers who patiently and eagerly participated in my long (up to 3 hours) interviews. I highly appreciate the time and energy that each of them generously put in my research as well as other researches related to water scarcity in Rafsanjan. Specifically, I would like to give my special thanks to Mr. Ahmad Alavi, chair of the Water Policy Research Institute. World would have been a much better place to live, if every policy makers were as passionate, concerned and truehearted as you are in solving the environmental problems. 
Writing this thesis would not have been such an enjoyable experience without my friends and colleagues in Netherlands. My special thanks go to Razieh, Flavia, and Divyani for accepting me in the "JOONS" group! I will never forget the funny, relaxing and joyful time that we had together. Many thanks to Edwardo and Shima for providing such great company in ITC lunch and coffee breaks and supporting me in all good and not-very-good days of my PhD. My thanks also to Nasrin, Mahshid, Soudi, Parisa, Nazanin and Pargol for all their warm-hearted care, support and true friendship over the 8 years of my life in NL. You girls have been my second family and made me feel home far from home. Additionally, I give my sincerest thanks to Sara.S for our amazing 14 years old friendship, and all the joyful chats and laughs that we shared over these years, no matter how far we have been from each other.

My sincerest thanks go to the PhD students and staff in ITC. It is hard to mention all of them, however, among them: Abhishek, Kwabena, Arun, Adish, Hamed, Soudabeh, Lidya, Rehana, Reshma, Sajel, Azar, Caroline, Yolla, Tina, Alby, Sheila, Gustavo, Tatjana, Parya, Andres, Alice, Simba, Hamideh, Siavash, Elham, Valentina, Shayan, Ieva, Sophie, Claudia, Samer, Fenna, Luc, Frans, Anna, Liza, Monika, Mila, Mafalda, Cheryl, Sherif, Johannes, Javier, Frank, Yola, Jaap, Karin, Chrit, Andre, Wilma, Monica, Christin, Dimo, Rohan, Roelof, Roshanak, Tony, Petra and Loes for being such amazing colleagues and friends in ITC.

My heartfelt thanks to Vahidoo, for being such a great inspiration in my life. I learned from you how to be happy and positive, believe in myself, follow my dreams, and never give up. Thank you for being by my side through all the moments of happiness and achievement as well as the moments of frustration and personal hardship. Nothing more than your support, presence and sincere believe in my potentials can give me the courage of doing what I like to do. Thank you for everything.

Last, but certainly not least, I thank my beloved family for always being supportive of all decisions that I made in my life and understanding me the best in the world. My lovely parents who sacrificed many things in their lives for me and my siblings, and made us who we are now. My love and respect for you two, Maman va Baba, are beyond the words. My wonderful brother, Amir, who never let me feel alone and always makes me laugh even in the toughest situations. My dear sister, Matin, for her kindness, compassion and warmhearted guidance over all the stages of my life. My thanks also to my brotherin-law, Morteza, and sister-in-law, Farinaz, for their support and kindness, my adorable niece, Nikoo, for bringing joy to our family, and my little nephew, Mani, who is supposed to be born on the same date of my PhD defence! I am forever thankful for being in this family. To you, I owe everything. 


\section{Table of Contents}

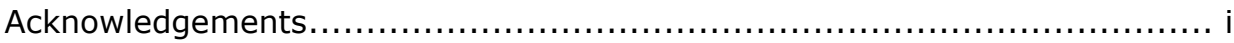

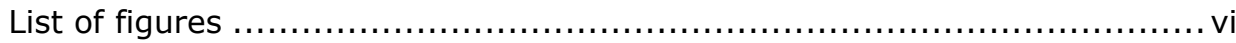

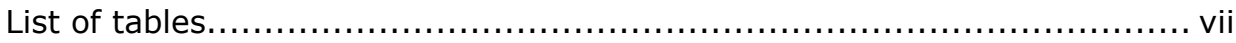

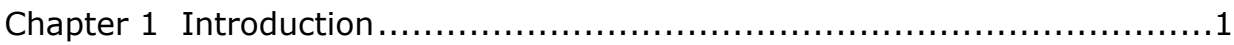

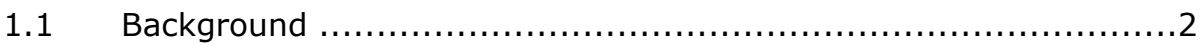

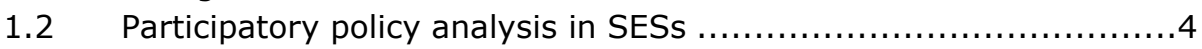

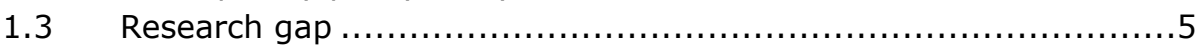

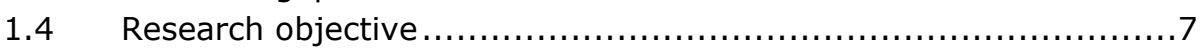

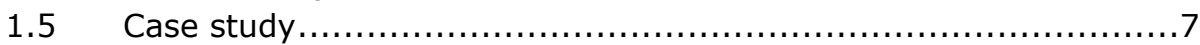

1.6 Outline of the research design and the thesis .......................8

Chapter 2 The Social-Ecological Analytical Framework of Water Scarcity in

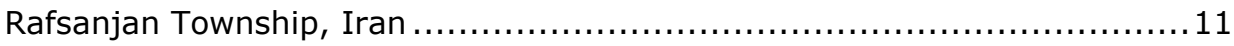

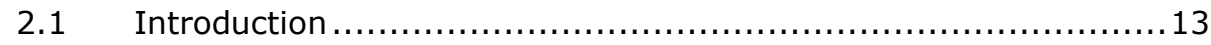

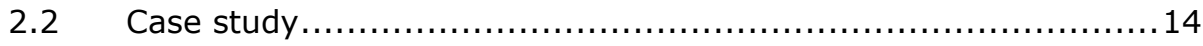

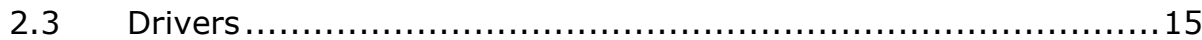

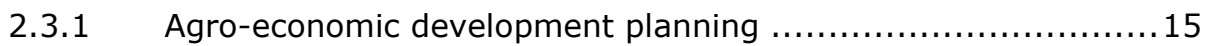

2.3.2 Population growth and urban expansion.......................... 16

2.3.3 Changes of water rights and their implementation $\ldots \ldots \ldots \ldots \ldots \ldots \ldots$

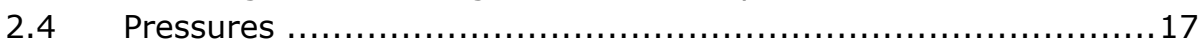

2.4.1 Agricultural land expansion (pressure on ecology) $\ldots \ldots \ldots \ldots \ldots \ldots 17$

2.4.2 Unregulated groundwater exploitation and well construction

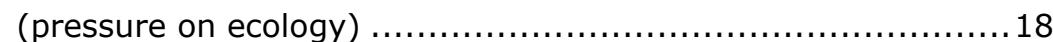

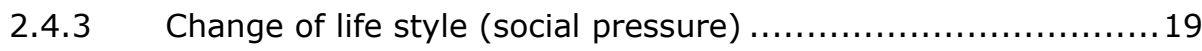

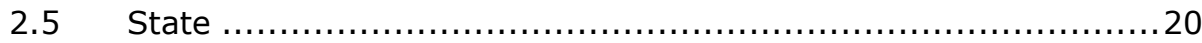

2.5.1 Decline of groundwater level (ecological state) $\ldots \ldots \ldots \ldots \ldots \ldots \ldots . . .20$

2.5.2 Land use change (vegetation area decrease) $\ldots \ldots \ldots \ldots \ldots \ldots \ldots \ldots .20$

2.5.3 Agricultural production and export decrease (economic state) .. 21

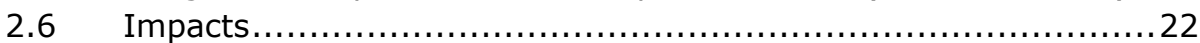

2.6.1 Critical ecology of Rafsanjan plain (ecological impact) ............22

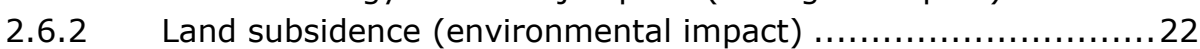

2.6.3 Vulnerable farmers and economy of the region (socio-economic

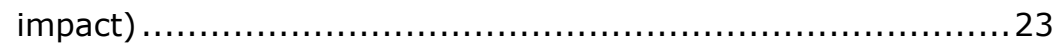

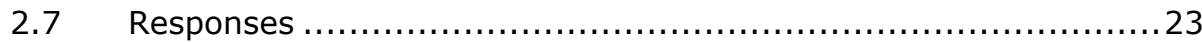

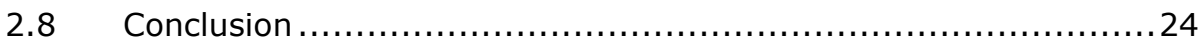

Chapter 3 A Structured Participatory Method to Support Policy Option Analysis

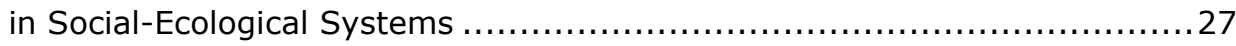

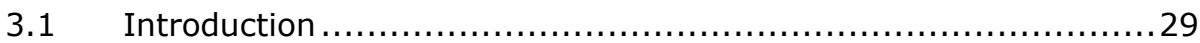

3.1.1 The Driver-Pressure-State-Impact-Response (DPSIR) ........... 30

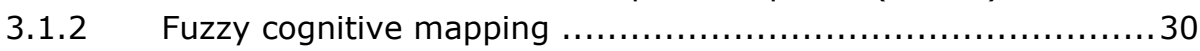

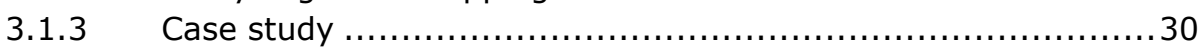

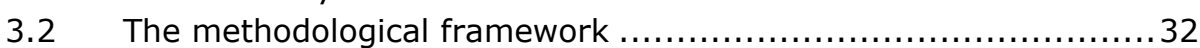

3.2.1 Step 1: Project objectives and system boundaries via DPSIR ... 32 
3.2.2 Step 2: Stakeholders knowledge modelling via FCM .............. 33

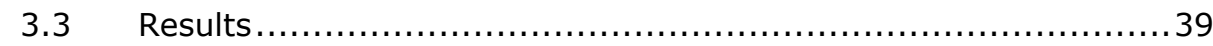

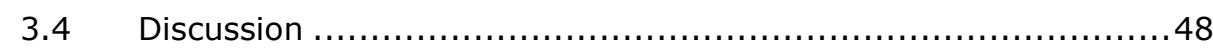

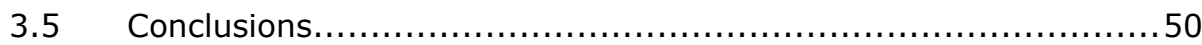

Chapter 4 Integrating Perception-Based and Data-Driven Knowledge to

Support Policy-Making in Social-Ecological Systems: A Fuzzy Cognitive

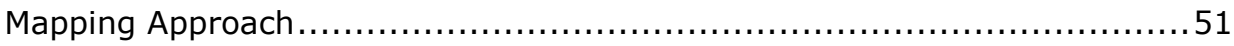

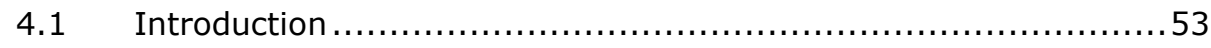

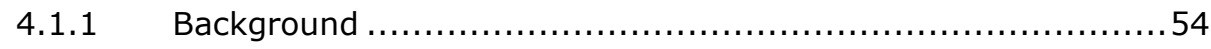

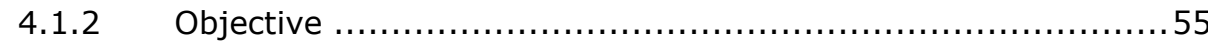

4.1.3 Technical background: Fuzzy Cognitive Map .................... 55

4.2 The Methodological Framework ................................... 56

4.2.1 Step 1: Define FCM structure via perceived knowledge...........57

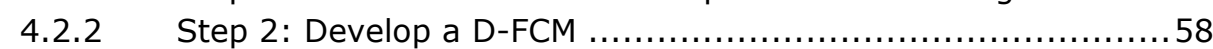

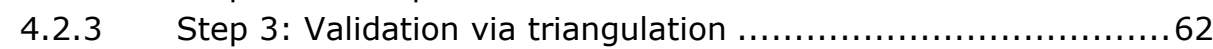

4.2.4 Step 4: Combine the D-FCM and P-FCM .......................62

4.2.5 Step 5: Simulate with the M-FCM model .......................... 62

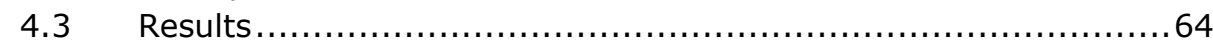

4.3.1 D-FCM structure, validation, and M-FCM structure ..............64

4.3.2 Policy option simulation with M-FCM model.....................69

4.3.3 Comparison of M-FCM and CP-FCM simulations ................ 71

4.4 Discussion and conclusion ...................................... 74

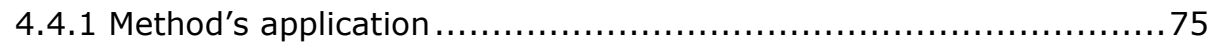

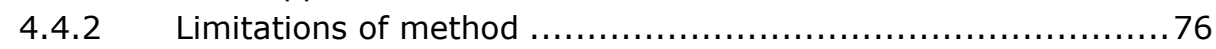

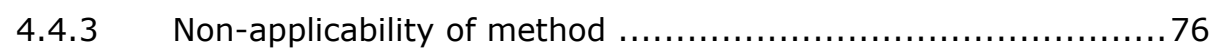

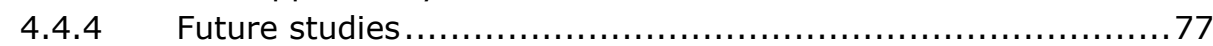

Chapter 5 From Individual Fuzzy Cognitive Maps to Agent Based Models:

Modelling Multi-Factorial and Multi-stakeholder Decision-Making for Water

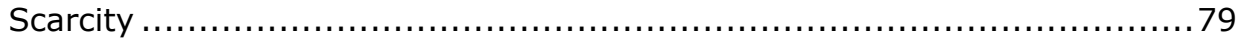

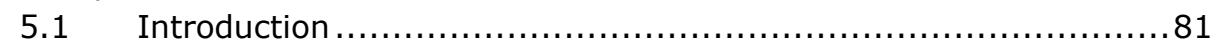

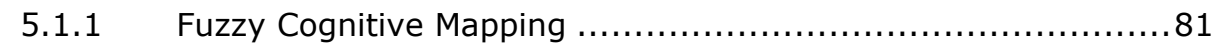

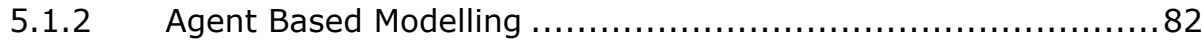

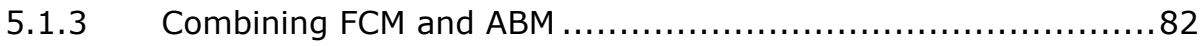

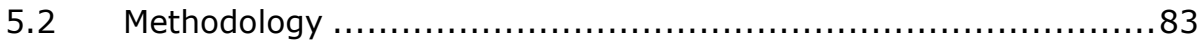

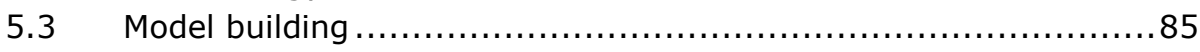

5.3.1 Case study: water scarcity and farming community adaptation in Rafsanjan, Iran ............................................... 85

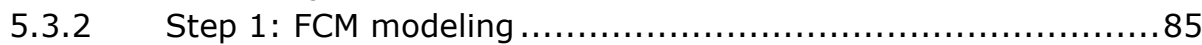

5.3.3 Step 2: Translating FCM to ABM .............................. 89

5.3.4 Step 3: ABM implementation and assessment.................. 96

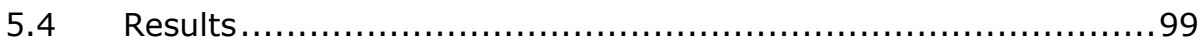

5.4.1 Baseline scenario.............................................. 99

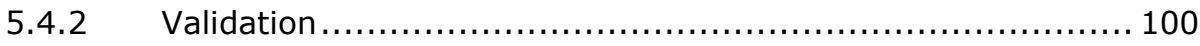

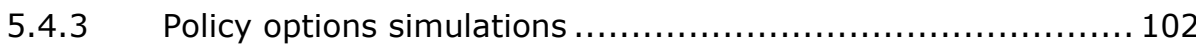




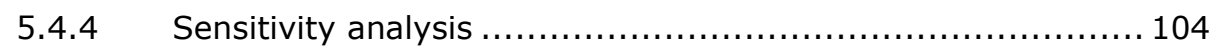

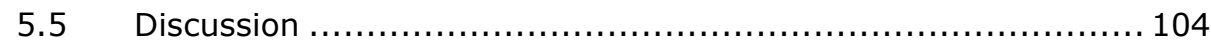

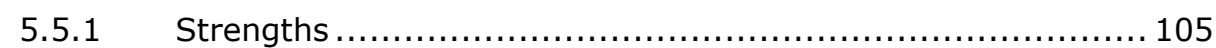

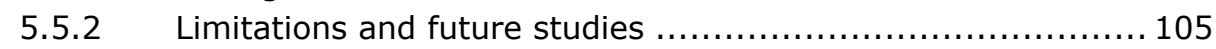

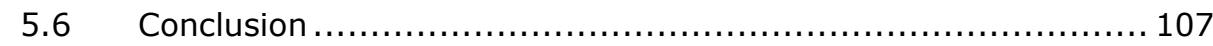

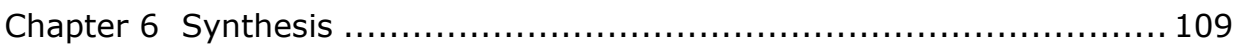

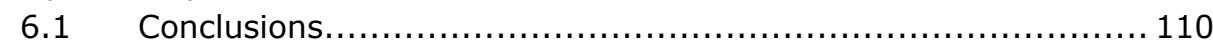

6.1.1 Objective 1: Developing an analytical framework to understand the social-ecological drivers and impacts of water

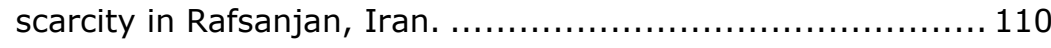

6.1.2 Objective 2: Integrating stakeholders' knowledge and perception in modelling SESs and evaluating acceptance of policies by stakeholders.

6.1.3 Objective 3: Integrating perception-based knowledge from stakeholders with data-driven knowledge from empirical studies in policy option analysis....

6.1.4 Objective 4: Simulating impacts of policy options by integrating multi-variables' behaviour and human interactions in SESs' modelling ........................................... 112

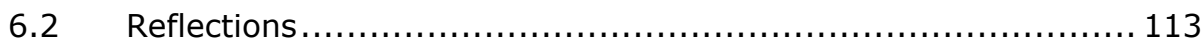

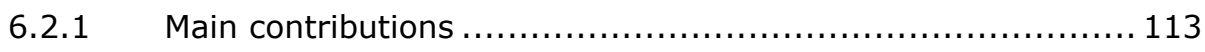

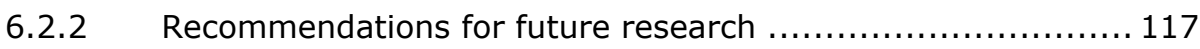

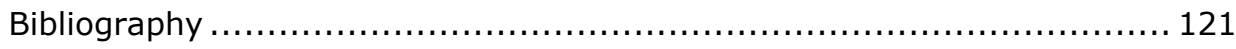

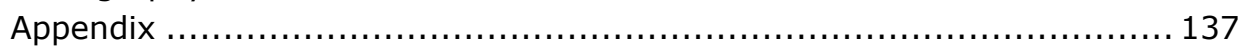

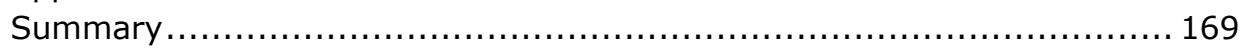

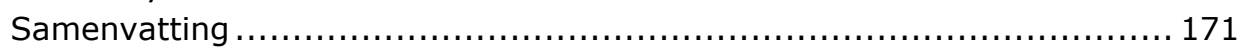




\section{List of figures}

Figure 1.1: Relations between human elements involved in participatory policy

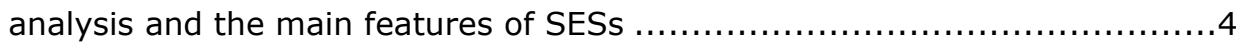

Figure 1.2: Four steps of the project and the connections between the steps..8

Figure 2.1: Location map of Rafsanjan Township........................... 14

Figure 2.2: Vegetation increase in Rafsanjan Township ..................... 17

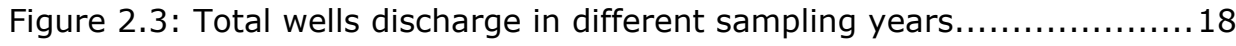

Figure 2.4: Ground water hydrograph of Rafsanjan Plain ...................... 20

Figure 2.5: Vegetation change detection in Rafsanjan Township ............. 21

Figure 2.6: Wells depth and ground displacement in areas of major land

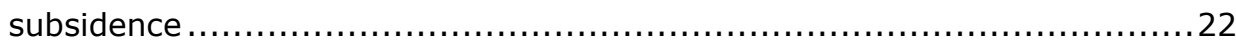

Figure 3.1: Rafsanjan Township in Kerman Province, Iran .................. 31

Figure 3.2: Preliminary DPSIR framework of water scarcity in Rafsanjan .... 38

Figure 3.3: Farmers' fuzzy cognitive map in DPSIR framework .............40

Figure 3.4: Policy makers' fuzzy cognitive map in DPSIR framework .........41

Figure 3.5: In-degree, Out-degree and Centrality of variables in farmers and

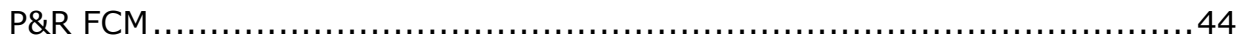

Figure 3.6: Simulation results of four policy options and their impact on each

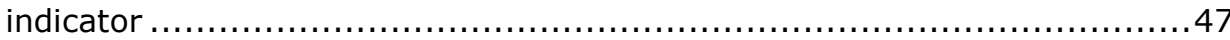

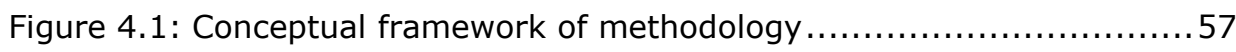

Figure 4.2: The CP-FCM of all stakeholders ............................... 59

Figure 4.3: Graphical representation of the D-FCM ........................65

Figure 4.4: Graphical representation of D-FCM and PP-FCM $\ldots \ldots \ldots \ldots \ldots \ldots .67$

Figure 4.5: Values of connections in three different FCMs: D-FCM, PP-FCM, FP-

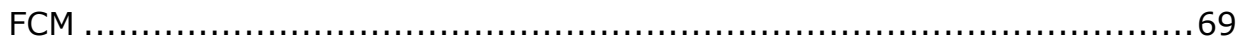

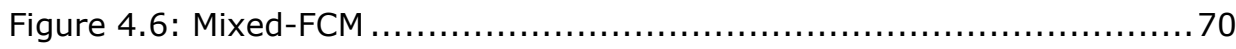

Figure 4.7: Results of four policy options simulation in M-FCM model and their impact on each indicator compared to the baseline steady state............ 72

Figure 4.8: Result of policy option simulation in A) M-FCM and B) CP-FCM

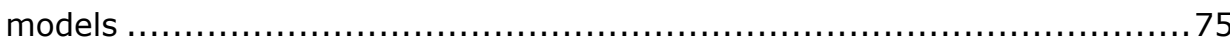

Figure 5.1: Main steps and sub-steps of methodology .................... 84

Figure 5.2: Group specific of large-farmers' FCM combined with objective data

Figure 5.3: Set-up and allocation of farmers and farms in Netlogo $\ldots \ldots \ldots \ldots . \ldots 9$

Figure 5.4: Condition-Action-Impact diagram of large farmers ................ 93

Figure 5.5: Comparing groundwater use in simulation and reality .............99

Figure 5.6: Groundwater use per region and overall groundwater use in three policy options scenarios compared to the baseline. 101

Figure 5.7: Number of large, medium and small farmers in three policy

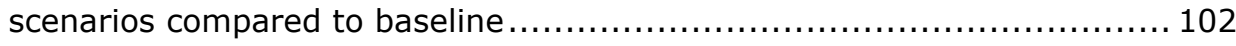

Figure 6.1: Four policy support methods, their required resources, and ability to capture complexity 


\section{List of tables}

Table 3.1: Interview questions and related DPSIR categories...................31

Table 3.2: Policy options, the clamped concepts and their values in each policy

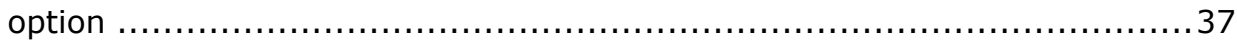

Table 3.3: FCM graph theory analysis per stakeholder group ..................45

Table 4.1: Description of variables with available time-series data.............60

Table 4.2: Policy options, the clamped concepts and their values in each policy

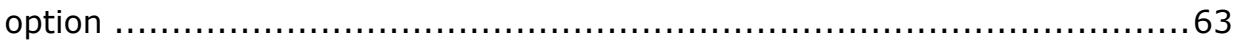

Table 4.3: Connections' weights in D-FCM, PP-FCM, and FP-FCM .............67

Table 5.1: Set of actions that can be taken by large, medium and small farmers 
Chapter 1

Introduction 


\subsection{Background}

Global warming and climate change are reflected in a variety of global environmental changes. The damage and loss of natural resources, including fish stocks, lakes, water resources, forests, and farms are noticeably increasing. Climate change adaptation is a response to the environmental consequences of climate change by attempting to reduce the vulnerability of social and ecological sub-systems to changes of temperature, rainfall, sea level, etc. (IPCC, 2007, UNFCCC, 2010). However, policy-making for climate change adaptation does not lead to useful results by focusing on either the social or the ecological systems separately. Instead, an integrated SocialEcological System (SES) perspective is required to provide insights on the interactions between human behaviour and environmental changes (i.e. social and ecological sub-systems) in climate policy analysis. SESs are known as complex, dynamic and uncertain systems (Berkes et al., 2000, Folke, 2006, Ostrom, 2007, Ostrom, 2009, Schlueter et al., 2012). Thus, a holistic climate policy analysis should deal with and address such integral features of the SESs (Walker et al., 2002, Levin et al., 2013). Out of several drivers discussed in literature, we point out three main social drivers of complexity, dynamics and uncertainty in the SESs:

The first drivers are the short- and long-term feedbacks between resources and stakeholders (users, policymakers, and managers) across multiple scales (Olsson et al., 2004, Cumming et al., 2006, Liu et al., 2007). For example, farmers of arable land learn in practice how to manage their groundwater use and deal with scarce water resources over a long period. Sound management may allow farmers to expand their farms. Government programmes may also support such expansion as it leads to the economic development of the region which, eventually, results in the massive growth of agricultural landscapes in the short-term. However, long-term impacts of climate change, i.e. increases in average temperature and reduction of rainfall, combined with short-term groundwater overexploitation from agricultural expansion may radically disrupt the agriculture production and economic situation of the region. Therefore, human decisions and actions to change one economic/environmental factor e.g. crop/animal farming may influence other environmental factors i.e. natural resources, which in turn encourage new human adaptive actions in the longer term. Such short-term and long-term feedbacks between human interventions and environmental changes accelerate the complexity and dynamics of SESs. Moreover, human adaptive decisions and actions in response to the environmental changes are usually not purely rational, can be very innovative, and are often more complex than being assumed or predicted (Johnson et al., 2017). Such unpredictable human responses give rise to the uncertainty of the SESs (Biggs et al., 2015). Therefore, climate adaptation policy analysis should consider the processes by which individuals or communities select and 
implement adaptive responses, and also how those choices affect or are affected by the environment (Adger et al., 2005, Adger et al., 2009). In other words, decisions and actions of stakeholders toward environmental changes should be taken into account for policy analysis in SESs.

The second source of complexity, dynamics and uncertainty in SESs exists in human interactions, mainly due to conflicts of interests and preferences between stakeholders. SESs often involve many stakeholders - natural resource users, policymakers, managers, etc.- with different and often conflicting interests and preferences which may lead to conflicts over the nature of the problem and potential solutions (Van den Hove, 2000, Hermans and Thissen, 2009). For instance, in a farming community, farmers' economic interests might be in conflict with the managers' environmental interests. Facing water scarcity, farmers might be in favour of increasing water accessibility solutions e.g. well deepening, water transfer, desalination, etc. whereas, managers might be interested in reducing water consumption solutions e.g. reducing farmed areas, water pricing policies, changing crops, etc. Moreover, stakeholders' values, interests and preferences may change over time based on their accessibility to information, interactions with other stakeholders and environmental changes. Therefore, heterogeneity in stakeholders' interests and preferences increase the dynamics and the uncertainty (Biggs et al., 2015) as well as the complexity in SESs (Van den Hove, 2000). In general it is agreed that in a multi-stakeholder SES better decisions are implemented with less conflict and more success when various actors' interests and preferences are involved in the process of policy analysis (Voinov and Bousquet, 2010).

Finally, complexity and uncertainty in SESs arise because the key drivers of SESs' changes such as climate and technological changes are fast and unpredictable phenomena for which we have imperfect and insufficient scientific knowledge (Adger et al., 2009). Even if the information exists, it is unlikely in a multidisciplinary SES that one actor covers all information. Rather, we require knowledge from various stakeholders who are in constant interaction with their environment and experience and observe changes in their environment. Therefore, SESs' policy analysis methods should be able to benefit from involving the knowledge and perceptions of local stakeholders about their environmental changes.

Figure 1.1 shows the lack of knowledge, stakeholders' conflicting interests and human-environment feedback as the main social sources of complexity, dynamics and uncertainty in the SESs. 


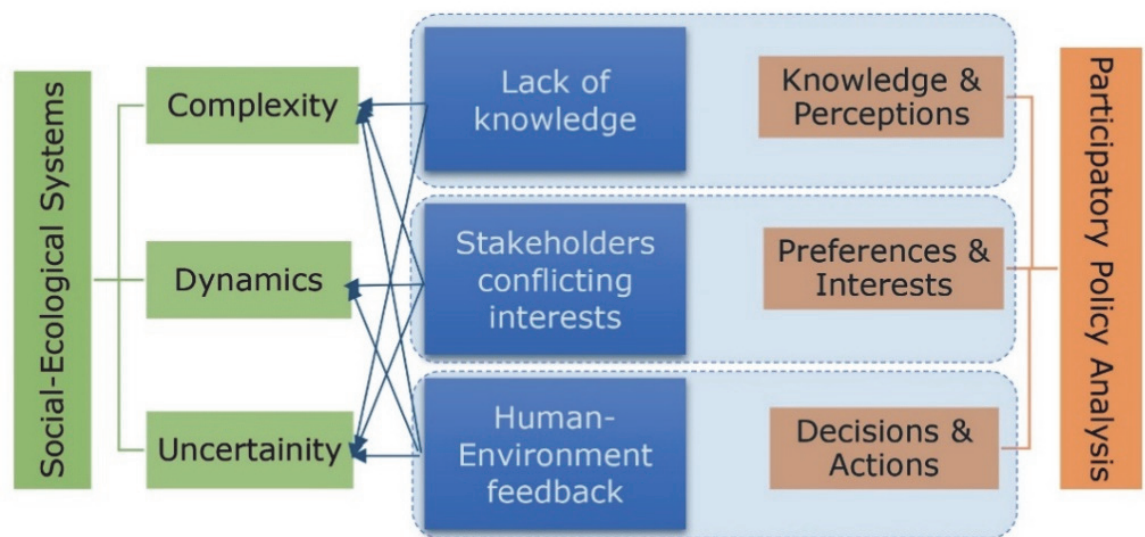

Figure 1.1: Relations between human elements involved in participatory policy analysis and the main features of SESs. Green box: SES features, Blue box: social drivers of each feature, Blue arrows: driver connections Orange box: human elements involved in participatory policy analysis, Dashed boxes: elements of participatory policy analysis that can address drivers of SES main features.

\subsection{Participatory policy analysis in SESs}

Participatory policy analysis methods have emerged to capture important features of a SES for policy-making (Durning, 1993, Holland et al., 1998, Durning, 1999, Geurts and Joldersma, 2001). These involve stakeholders' knowledge, perceptions, preferences, and decision-making in the process of policy option simulations. Primarily, participatory policy analysis has evolved in response to the failures of traditional policy analysis approaches that place more emphasis on formal (quantitative) modelling, rational planning and cost-benefit analysis to find optimal solutions for all sorts of complex problems (Geurts and Joldersma, 2001). Such approaches follow a conventional "positivist" and "rational" view on policy-making, which has two main assumptions: 1) knowledge about the reality should be separated from subjective human judgments; therefore, policy analysis should rely on objective knowledge and data based on scientific research, and 2) policy analysis should support rational policy-making, in which actors choose the optimal means to achieve given objectives. These assumptions suggest it is possible to determine the "best means" or "optimal solution" per case (Monnikhof, 2006). However, the solutions of such approaches are frequently criticized as being anti-democratic, too general, and too narrowly focused on a means-end rationality (Hermans and Thissen, 2009).

By contrast, participatory policy analysis emphasizes the involvement of a diversity of knowledge, beliefs, preferences, and values of the people managing or depending on a SES in identifying the problem and developing the solution (Lynam et al., 2007). Several overlapping ideas have been mentioned in literature on how participatory policy analysis can help effective 
policy-making in SESs. First of all, incorporating local stakeholders' perspectives allows pooling of information and helps to solve the problem of information and knowledge scarcity, which goes hand in hand with uncertainty in SES policy analysis (Adger et al., 2009). Secondly, participation of different stakeholders in policy analysis can potentially lend a higher degree of legitimacy to the decisions taken since a wider range of stakeholders' interests has influenced the policy choice process. Moreover, a policy that is well grounded in stakeholders' perceptions, preferences and interests might be implemented in a smoother and less costly manner because stakeholders are expected to be more cooperative in the implementation of policies they have helped to create (Irvin and Stansbury, 2004, Nuno et al., 2014). Thirdly, participatory policy analysis helps policymakers to understand the degree of acceptability of the policies by stakeholders or their possible responses to the policies, before their implementation.

Involving different stakeholders' knowledge, perceptions, preferences, and decisions can address the most important features of an SES i.e., complexity, dynamics and uncertainty, in policy-making by taking into account the main social drivers of such features (figure 1.1).

\subsection{Research gap}

Despite increasing attention for the theories and application of participatory policy analysis in the management of SESs some research gaps can still be identified.

1. Lack of systematic methods to involve qualitative knowledge in policy analysis:

Getting qualitative evidence into policy-making processes and communicating qualitative findings to policymakers is a key challenge worldwide (Davies and Nutley, 2000). Qualitative evidence has often been seen as too subjective, biased, unreliable, unscientific and context-specific to be used for policy analysis (Yang and Gilbert, 2008, Edmonds, 2010). This assumption is because qualitative data can have many different interpretations, which may result into many different solutions. Researchers working with qualitative data usually apply their own interpretive judgments to draw general conclusions based on collective qualitative data (Gilson, 2014). Various methods have been developed to collect and represent the qualitative knowledge of stakeholders for the policy analysis e.g. role-playing games (Pak and Brieva, 2010), focused group discussion (Kahan, 2001), and in-depth semi-structured interviews (Van Audenhove, 2007). Yet, how to systematically structure and formalize the informal and subjective stakeholders' voices for use in policy-making processes remains a key challenge (Sun and Müller, 2013). 
2. Lack of methods to integrate qualitative and quantitative evidence:

The literature recognizes that both qualitative and quantitative evidences are required for modelling SESs (Ostrom, 2009), but integrating qualitative and quantitative evidences in SES modelling remains challenging, as both have their characteristics, strengths, and weaknesses. Mathematical and equationbased models-, which have been used more often for ecological, and natural resource modelling-fit with quantitative data naturally. Qualitative data, on the other hand, fit with non-numerically based models (Edmonds, 2010). As a result, qualitative and quantitative methods and tools are often used separately depending on the objectives of the research (López-Ridaura et al., 2002, Poteete and Ostrom, 2005, Arcidiacono et al., 2009). Many ecological studies focus more on quantitative methods and social studies are more likely to include qualitative methods. Therefore, the lack of methods that integrate both types of qualitative and quantitative evidence in modelling leads to difficulties in implementing interdisciplinary SES studies.

3. Lack of methods to represent perception, preferences, behaviour and feedback of stakeholders in one model.

Some participatory modelling methods focus on collecting human knowledge and perceptions and use them as input data for developing the SES models e.g. participatory Fuzzy Cognitive Mapping (FCM) (Özesmi and Özesmi, 2003), Participatory System Dynamic Modelling (PSD) (Stave, 2010, Zimmerman et al., 2016) and participatory Bayesian Belief Network (BBN) (Salliou et al., 2017). Some others focus on simulating human decision-making and their feedbacks to the system over time by using rational choice theory or survey data to predict human behaviour e.g. Agent-Based Modelling (ABM) (Gilbert, 2008). However, there is a need to understand and incorporate all human elements-e.g. perceptions, preferences, decisions and actions-into SES modelling for policy-making (Elsawah et al., 2015). This need has been motivated in literature by the argument that human perceptions, decisions and actions (altogether) influence and are influenced by changes in the environment and natural resources directly and indirectly. Managing SESs, therefore, is more about managing human behaviour rather than managing the environment (Ludwig et al., 1993). Consequently, policymakers need to understand and change factors that influence how people perceive problems and the solution space, how they make decisions, and how they implement their decisions. A key challenge to address this need is bridging the gap between knowledge co-production methods and actors' behaviour modelling methods.

This research addresses these gaps. 


\subsection{Research objective}

The main objective of this research is to develop and demonstrate participatory policy analysis methods to support policy-making in SESs' environmental challenges. The research is built upon a case study of a farming community facing water scarcity in Rafsanjan, Iran (see the following section for a brief introduction to the case study area, and chapter 2 for a detailed description). To achieve this objective, the following specific objectives have been defined:

1) To develop an analytical framework to understand the social-ecological drivers and impacts of water scarcity in Rafsanjan, Iran.

2) To integrate stakeholders' knowledge and perceptions in modelling the SESs and evaluating stakeholders' policy acceptance.

3) To integrate perception-based knowledge from stakeholders with datadriven knowledge from empirical studies in policy option analysis

4) To simulate impacts of policy options by integrating multi-variables' behaviour and human interactions in SESs' modelling

\subsection{Case study}

Rafsanjan Township is the biggest producer of pistachio in Iran, one of the two top producers and exporters of pistachio in world, the other being the USA. Due to their high value, pistachio exports have a significant share in non-oil revenues of Iran, so that it is sometimes termed the "green gold" of the country. However, pistachio production is currently threatened by growing concerns about water scarcity.

Water scarcity in Rafsanjan is a social-ecological problem. Both climatic change (i.e. the lack of precipitation and increasing heat), and human interventions (i.e. decades of water and environmental mismanagement, agricultural expansion, and ground-water overexploitation) play substantial roles in the water scarcity. Moreover, both social and ecological variables are influenced by water scarcity in Rafsanjan (e.g. land cover, aquifer sustainability, land subsidence, pistachio production, the economy of the region, farmers' vulnerability, emigration, etc.). To combat water scarcity in Rafsanjan, the Iranian Government has suggested some policy options (e.g. shrinking the farms' area, irrigation/agriculture system modification, economic change, government control and monitoring, increasing people participation, etc.). However, each policy may have uneven impacts socially, spatially and temporally. The possible impacts of such policies on different stakeholders, various locations and over the short and long period should be simulated, before their implementation. Creating a methodology to address these issues is the subject of this research. Further details of the case study are provided in chapter 2 of the thesis. 


\subsection{Outline of the research design and the thesis}

This research was conceived as a series of four steps, from problem structuring, to knowledge acquisition, integration of qualitative and quantitative evidences and finally modelling the SES for policy impact evaluation. The participatory methods used and their sequence were designed to integrate stakeholders' knowledge, perception, preferences and decisionmaking in the process of policy analysis in SESs.

For this purpose, a combination of DPSIR framework (Drivers, Pressures, State, Impacts, and Responses), Fuzzy Cognitive Mapping (FCM), and AgentBased Modelling (ABM) methods are developed and applied in the case of policy analysis for water scarcity in Rafsanjan, Iran. DPSIR is a problem structuring method (Kristensen, 2004) used in step 1 for categorizing and structuring the complex SES problem of water scarcity in the case study. In step 2, the DPSIR framework is combined with a participatory FCM, i.e. a knowledge coproduction method (Özesmi and Özesmi, 2004), to systematically collect the knowledge and perceptions of stakeholders on the data-scarce part of the system and to represent these in a semi-quantitative model. In step 3, the qualitative knowledge produced by participatory FCM is combined with quantitative data to develop a mixed-FCM representing complex SESs. In step 4 , the outcome of the mixed FCM is used as an input to develop an ABM (Gilbert, 2008) to model the decisions and actions of stakeholders and simulate the macro-level patterns of the system that emerge from individual behaviour (figure 1.2). Finally the impact of government policy options are simulated by integrating knowledge, perceptions and preferences of stakeholders represented in FCM models, and their decisions and actions represented by ABM. 


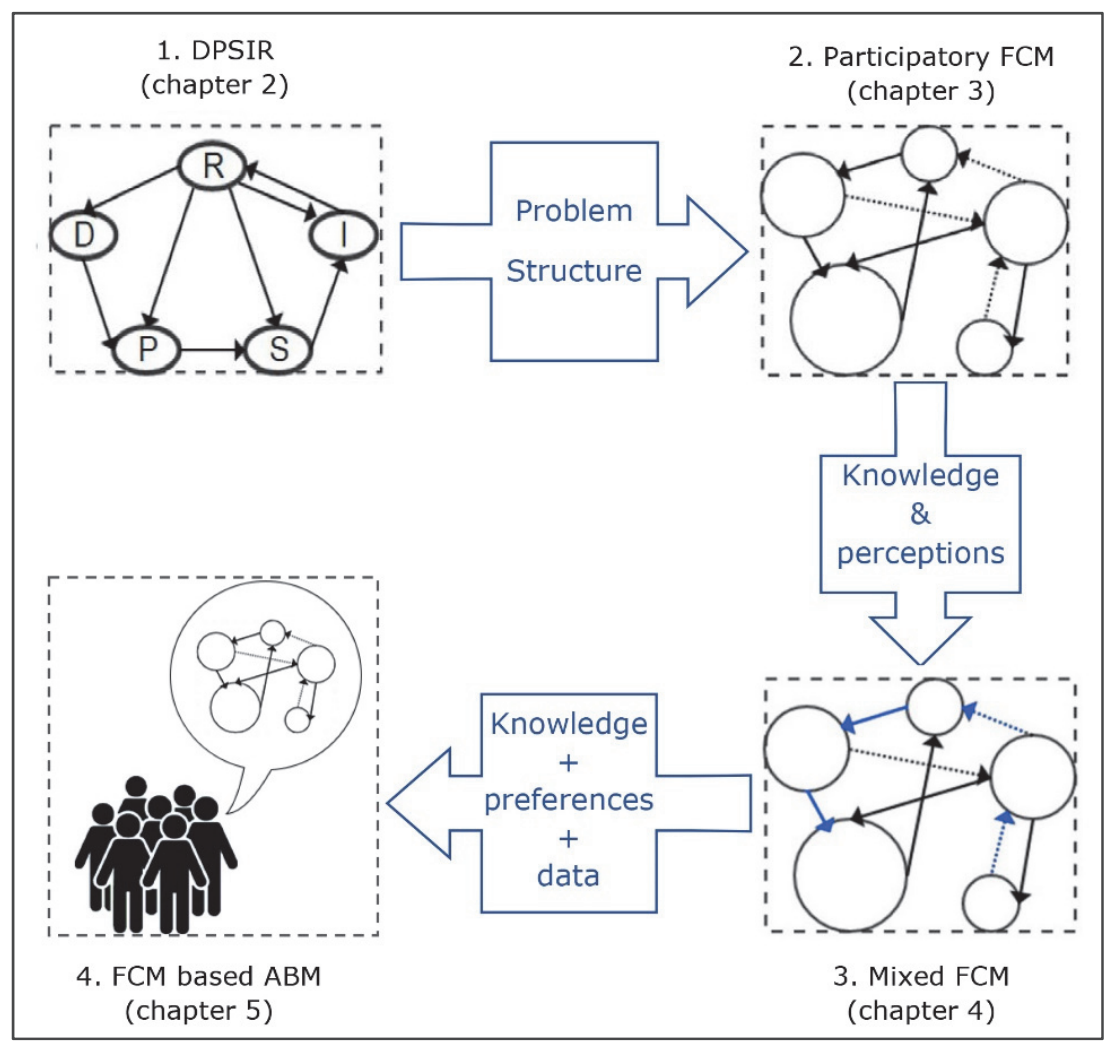

Figure 1.2: Four steps of the project and the connections between the steps

This thesis consists of six chapters. The four core chapters (2-5) correspond to the research findings related to each of the objectives mentioned above. Details of the methods for each objective, and their connections and combinations are also described in the chapters 2-5 that represent articles published in or submitted to international journals.

Chapter 1 presents a brief research background, the rationales for the selection of research objectives and outline of the thesis.

Chapter 2 addresses the first objective of the thesis. This chapter identifies, categorizes and presents the leading social and ecological causes and effects of water scarcity in Rafsanjan, Iran by using DPSIR framework. Inputs are generated through a time series analysis of Landsat images, extracted NDVI images, secondary literature, government reports and census data. This chapter provides an analytical social-ecological framework that will be used as input for the next chapter.

Chapter 3 deals with the second objective of this thesis. This chapter sets the ground for involving different stakeholders' knowledge and perception in 
modelling SESs. FCM is used to collect and present the perceptions of 60 farmers and 40 policymakers over causes and impacts of water scarcity in Rafsanjan, Iran. The DPSIR framework developed in chapter 2 is used to structure and categorize complex FCMs to be used for policy analysis processes. The outcome of this chapter is a participatory mixed-method that is used for comparing perceptions of farmers and policymakers over the problem and possible solutions, and simulate impacts of policies based on stakeholders' knowledge-i.e. acceptance of policies by different stakeholders.

Chapter 4 addresses the third objective of this thesis. This chapter presents a methodological approach to develop FCM by combining qualitative and quantitative evidence. The stakeholders' perception provides qualitative data for social aspects-e.g. farmers' vulnerability, adaptation strategies, participation in groundwater use management, etc.-and empirical studies provide data for ecological aspects-e.g. precipitation, groundwater level, land subsidence, etc. The introduced methodology combines these two types of knowledge in one FCM model. The outcome of this chapter is a qualitativequantitative modelling method useful for analysing policy options in SESs in which both kinds of knowledge are important and can be complementary.

Chapter 5 presents the research related to the fourth objective. This chapter presents a methodology to 1) present stakeholders' knowledge, perceptions, decisions, and actions facing a social-ecological problem, and 2) simulate impacts of policies considering all these human elements. FCM is used to translate qualitative evidence to be used as behavioural rules in $A B M$ development. Therefore, FCM represents the stakeholders' knowledge, perceptions, and preferences, and ABM simulates farmers' decision-making and impacts of their actions on the overall groundwater use in Rafsanjan, Iran. The outcome of this chapter is a participatory modelling method that covers the main features of a SES i.e. complexity, dynamics, and uncertainty for policy options analysis.

Chapter 6 represents the main research findings and conclusions corresponding to each research objective. The main contributions and novelty of scientific output of this thesis are discussed followed by recommendations for future research to improve participatory modelling further to support policymaking in SESs. 


\section{Chapter 2*}

\section{The Social-Ecological Analytical Framework of Water Scarcity in Rafsanjan Township, Iran}

\footnotetext{
* This Chapter is based on:

Mehryar, S., Sliuzas, R., Sharifi, A. and van Maarseveen, M.F.A.M., 2016. The socioecological analytical framework of water scarcity in Rafsanjan Township,

Iran. International journal of safety and security engineering, 6(4), pp.764-776.

Mehryar, S., Sliuzas, R., Sharifi, A. and van Maarseveen, M.F.A.M., 2015. The water crisis and socio-ecological development profile of Rafsanjan Township, Iran. WIT Transactions on Ecology and the Environment, 199, pp.271-285
} 


\begin{abstract}
Ground water scarcity is a main socio-ecological challenge in the Middle East. While ground water reserves seem vast, the impacts of over-exploitation and inadequate control over water consumption may threaten the sustainability of aquifers. The signs of aquifer depletion and its influence on water accessibility have become apparent in recent years. Using the case of Rafsanjan Township, Iran, this study aims to understand the socio-ecological factors and their interrelationships in driving and exacerbating the water crisis situation, the ongoing policy responses and the possible consequences of current trends. The DPSIR framework (Drivers, Pressures, State, Impacts and Responses), developed by the European Environmental Agency in 1999, is used to analyze the components of the socio-ecological system. Inputs are generated through a time series analysis of Landsat images, extracted NDVI images, secondary literature, government reports and personal interviews with local experts.

This study illustrates the conflict between rapid economic development policies that have simulated the expansion of pistachio orchards on the one hand and sustainable water resource management on the other. Some responses based on a long-term socio-ecological resilient planning approach may provide a more sustainable perspective, but will require a substantial rethinking of current policies, improved water management practices, and additional research.
\end{abstract}

Keywords: water scarcity, socio-ecological system, DPSIR, water resource management. 


\subsection{Introduction}

Rafsanjan Township, with a population of more than 280,000 people can be seen as a highly stressed socio-ecological system in which diminishing water resources is a decisive factor. This paper examines the socio-ecological variables of environmental changes due to the water crisis in Rafsanjan, and possible policy consequences. The current water scarcity in Rafsanjan is derived from long term ecological and social challenges. Rafsanjan is located in one of the world's drought-prone areas, with an average annual precipitation of $137 \mathrm{~mm}$, equivalent to one-sixth of the world average precipitation (IWPRI, 2014). Besides the periodic droughts and effects of climate change, Rafsanjan, like many other parts of Iran, has been under the pressure of socio-economic shocks after the oil industry growth, white revolution and 1979 revolution of the country (Alizadeh and Keshavarz, 2005, Madani, 2014). Rapid population growth and economic instability after the revolution and oil price change persuaded the Iranian government to implement economic development plans, supporting the industrial and agricultural sectors, to satisfy growing demand in the country and to make the country more self-sufficient (Gilanpour, 2006, Khorami and Pierof, 2013, Metz, 2013). However, the thirst for rapid economic development has largely ignored the negative impacts on natural resources by excessive concentrations of population and land uses throughout the potentially agricultural and industrial areas.

Rafsanjan as the main center of pistachio production and export in Iran has witnessed the accelerating expansion of agriculture derived by the economic development strategies. However, the long-term negative impacts of former development plans and their incompatibility with the available water resources are now being revealed. To illustrate the scale of the problem and the importance of a better integration between development policies and environmental resource management, this paper presents a detailed descriptive analysis of the changing socio-ecological system in Rafsanjan Township and particularly the increasing water scarcity.

The European Environment Agency's Driver-Pressure-State-Impact-Response (DPSIR) framework (Kristensen, 2004) is used to review the complex and causal relationships between key factors that contribute to water scarcity in Rafsanjan Township. Driving forces here are the socio-economic development and changes in society resultant changes in governing system and also peoples' lifestyles. Agro-economic development, oil-reliant economic development, population growth and change of water laws and legislations are among the main drivers examined in this paper. These driving forces lead to direct or indirect pressures that disturb the normal state of the environment. Agricultural expansion, unregulated groundwater exploitation and well construction, and change of lifestyle are described as the main pressures. 
These pressures in turn affect the state of the environment that is described by trends and the current situation. The state variables in this paper are declining groundwater levels, agricultural production reduction, land use change, and land subsidence. Changes in a state may generate social and ecological vulnerabilities, which are defined as impacts. The impacts of water crisis in Rafsanjan is explained in three aspects of critical ecology of Rafsanjan Plain, land subsidence and vulnerable farmers' community. Finally, responses are the actions taken by society or policy makers to improve the situation. The responses might be toward any part of the chain between driving forces and impact. The responses to combat the water crisis in Rafsanjan are described from water supply and demand control perspectives. Secondary data used in the analysis were collected from literature, government reports and official data. Primary data were derived by using remote sensing image analysis (Landsat images of 28 years in time series of 1986, 1998, 2009 and 2014) as well as several informal interviews in October 2014 with key informants from the private sector, NGOs and the governmental water authority.

\subsection{Case study}

Rafsanjan Township consists of four districts, five urban areas, 14 dehestan (above village level) and 305 villages occupying $8288 \mathrm{~km} 2$ or $4.5 \%$ of the Kerman Province (Figure 1). The city of Rafsanjan, located at $30^{\circ} 30 \mathrm{~N}$ and $55^{\circ} 40 \mathrm{E}$, with a population of 151,000 (Census 2011), is the township's largest city. Rafsanjan Township and Kerman Province are the main producers and exporters of pistachio in the world. Pistachio, termed the green gold of the country, is the main identity of this region. Almost $96 \%$ of agricultural lands in the township is allocated to the pistachio crop (Jamab, 2011). However, the growing concern with water scarcity is threatening the economic base of the region, its identity and its sustainability. 


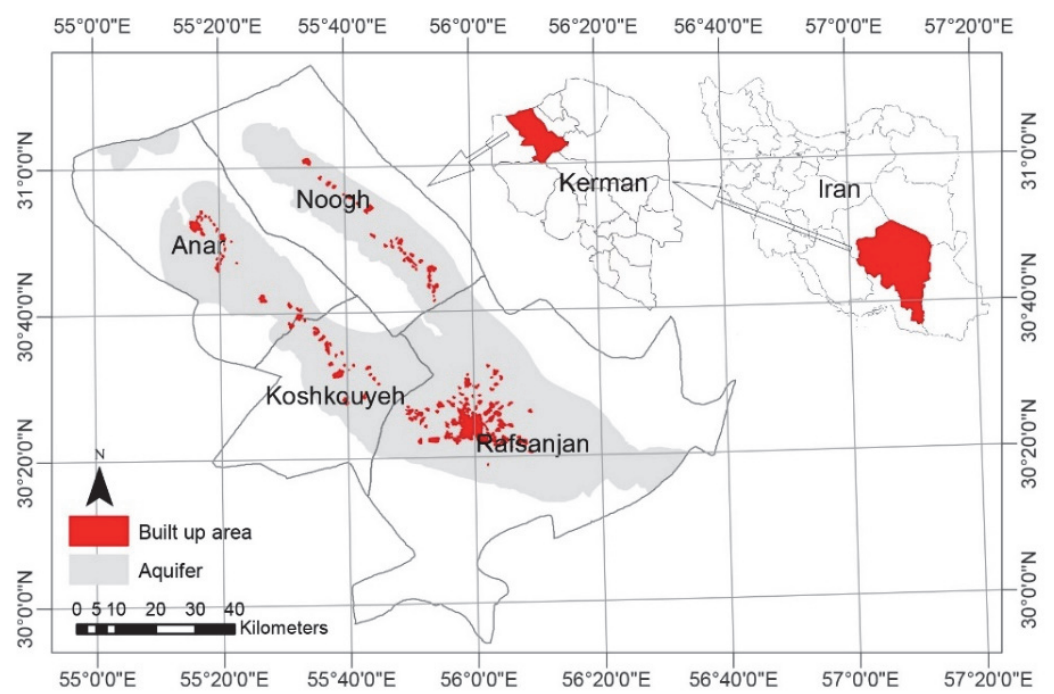

Figure 2.1: location map of Rafsanjan Township in province and country, derived from Landsat 8

\subsection{Drivers}

\subsubsection{Agro-economic development planning}

Agriculture has always been prioritized by the Iranian government as the main engine of national economic development (Shakoori, 2006). After the 1978 Revolution, the new Islamic Republic government used the term "Agriculture Jihad" (holy war), to satisfy the dramatically increasing food demand and also to improve the self-reliance of country (Kalantari, 2015). Subsidizing of water and energy for the agricultural sector and importing higher technology of pumping wells were among the supportive actions of government to encourage agricultural expansion without considering the available natural resources of the country (Shakoori, 2006, Madani, 2014).

Another decisive factor in agro-economic change in Iran was the rapid increase of oil revenue which is known as the "Dutch disease". This term is used when a country discovers a valuable natural resource deposit and begins large-scale exploitation of it and its revenues. As a result, the country's currency gains value (David, 1996), making the import of foreigner production more profitable. Over-dependency on a single export product makes the economy of a country highly vulnerable to that product's price fluctuations. In Iran, to overcome the economic shocks of oil price changes, the government adapted the dual exchange rate and put subsidies on water and energy for farmers (Farzanegan and Markwardt, 2009). Therefore, the large exchange rate difference in conjunction with cheap energy encouraged the farmers of Rafsanjan to import more pumping wells in order to expand pistachio 
production. The pump technology that was formerly unavailable and unaffordable became more beneficial and gradually replaced the traditional Qanat system for exploiting groundwater for irrigation (Mehryar et al., 2015). Moreover, together with water and energy subsidies the high exchange rate for exporting pistachio made the pistachio production highly lucrative for the farmers in Rafsanjan (Agah and Hassani, 2014).

\subsubsection{Population growth and urban expansion}

In the 20 years after the 1978 Islamic Revolution, due to the government's policy to stimulate Islamic population growth and to ban birth control, the country's population increased from 34 million to 63 million (Roudi-Fahimi, 2002). This "baby boom" contributed to a dramatic increase in water demand and so a reduced supply of renewable fresh water per capita (Madani, 2014). Increased food demands and employment stimulated the government to further develop the agricultural sector.

The population data from the Statistical Centre of Iran (SCI, 2014) show that Rafsanjan Township experienced a high population growth after the Islamic Revolution too, with an annual growth rate of $2.5 \%$ between 1986 and 2006 . This process is ongoing and based on 2009 census data it is estimated that by 2024 the urban population of Rafsanjan will have increased with 38\% (RDPI, 2009).

\subsubsection{Changes of water rights and their implementation}

Water laws and their enforcement have also played an important role in excessive agricultural water use in Rafsanjan. Traditionally, the farmers were used to invest in drilling wells for the operation and management of ground water. The utilization of water resources were governed by shari'a law (complex body of Islamic rules) and customs (Alasti, 2013). By the enactment of the Nationalization of Water Resources Act (NWRA) the underground water resources were considered as national property and their utilization and management were charged to the Ministry of Energy. In spite of many potential advantages of changing water governance from private to public goods, the water nationalization laws reduced the water users' participation. Since the rights of water users were not clearly defined by the NRWA, farmers perceived the new centralized water governance as an ownership loss and gradually lost their incentives to consider the aftereffects of their water over-extraction (Agah and Hassani, 2014).

Another important water law that came into effect after the revolution was the Equitable Water Distribution Act (EWDA). As part of this law the definition of "prohibited plain" was identified based on judgments of two experts of the water authority (Agah and Hassani, 2014). Earlier, the prohibited plains were defined according to the water balance of aquifers, meaning the aquifers with 
negative balance of ground water inflow and outflow would be called "prohibited plain" where well drilling and well discharge were forbidden. However, after EWDA many plains could get the discharge licence despite their negative aquifer imbalance based on these experts' judgements. In the rather chaotic situation after the revolution, populistic action of the policy makers and the corruption in governmental sectors exacerbated the over exploitation of groundwater in Rafsanjan. Apparently both small-holder famers and large landlords could highly benefit from this situation but only by depreciating critical communal ground water resources.

Moreover the misinterpretation of Islamic jurisprudence caused easier justification of excessive ground water exploitation. As an example, according to the Islamic water law the digger of a well, whether on his land or on unoccupied land, becomes the owner of the well water as soon as digging is completed (Caponera and Nanni, 1992). After the revolution many wells' discharges were licenced based on Islamic water laws and populistic actions of revolutionary politicians (Agah and Hassani, 2014).

However, the vague water laws and legislations and their weak implementation and supervision, facilitated the violations, law breaking and water theft in Rafsanjan. According to the FAO Water description (Water, 2007), for most local water users, water scarcity is not only about drought or wells drying up but above all it is about their fair and safe access to water. It is about how governments guarantee the equity, transparency and monitoring of law enforcement which has already been ignored through weak ground water governance in Rafsanjan.

\subsection{Pressures}

\subsubsection{Agricultural land expansion (pressure on ecology)}

Excessive agricultural area expansion is a direct pressure inflicted by the socioeconomic drivers of development planning and population growth. To explore agricultural land expansion within the Rafsanjan Township, we prepared the NDVI images derived from Landsat satellite images over a 28 years period between 1998 and 2014 (Mehryar et al., 2015). NDVI images are extensively used in determining water stress levels on vegetation and assessing drought impact (Peters et al., 2002, Al-doski et al., 2013, Gopinath et al., 2015, Himanshu et al., 2015). Higher values of NDVI indicate healthier vegetation while unhealthy or sparse vegetation shows a lower value. These data show that the pistachio land area within the Rafsanjan Township has grown dramatically over recent decades. Around 35,000 ha were added to the vegetated area over this period, which is almost one third of current planted area. Most agricultural expansion (52\%) occurred between 1998 and 2009, despite the drought that had started from 1999. Most of the agricultural 
expansion is located around Rafsanjan City in the south and the Noogh region in the east (Figure 2).
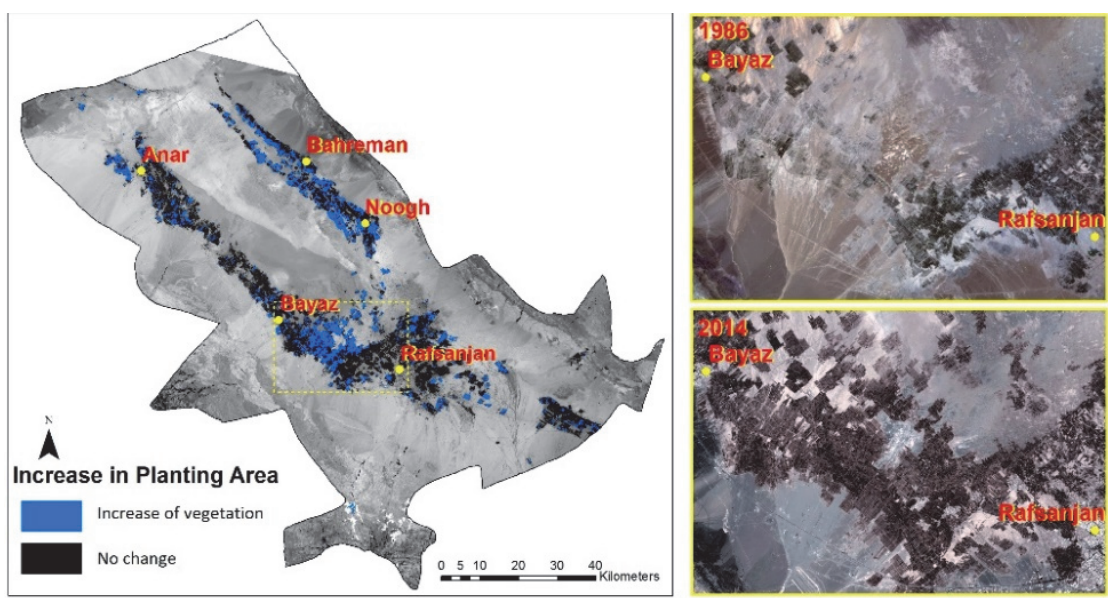

Figure 2.2: Vegetation increase in Rafsanjan Township between 1986 and 2014 (Mehryar et al., 2015)

\subsubsection{Unregulated groundwater exploitation and well construction (pressure on ecology)}

From 1970 till 2006, the number of wells in Rafsanjan township increased from 590 to 1392 (IWPRI, 2014). Data indicate a striking increase of well drilling between 1971 and 1983 which is after the water nationalization, land reform and boom of oil trade in Iran. However, these data refer to legal wells while, according to local knowledge, the number of illegal wells drilled over the last 30 years even exceeds the number of legal wells.

The rapid increase of deep wells further reinforced the high pressure on groundwater exploitation creating a water imbalance in the aquifer. In 1974 Rafsanjan Plain, for the first time, was declared as "prohibited plain" due to a $20 \%$ overexploitation of groundwater (RDPI, 2009) with an annual well discharge of 334 million $\mathrm{m}^{3}$. Nevertheless, the statistics of groundwater discharge in Rafsanjan plain (Figure 3) show that water extraction reached 780 million $\mathrm{m}^{3}$ in 1993, more than twice the amount of 1974. According to the most recent hydro-geological report (MPO, 2003), Rafsanjan's aquifer had 37\% overexploitation of the groundwater balance and $22 \%$ overexploitation of its permissible level. The government has set the permissible annual extraction of 559 million $\mathrm{m}^{3}$ for this aquifer, while its actual annual extraction is recorded as 685 million $\mathrm{m}^{3}$ (Jamab, 2011). In 2009 the groundwater balance of Rafsanjan was -215 million $\mathrm{m}^{3}$ (RDPI, 2009). It is estimated that Rafsanjan has already used most of its groundwater resources and its aquifer has been classified as a "critical prohibited plain" by the government since 2006; implying that further well drilling in the region is not allowed and that groundwater 
withdrawal from licensed wells should be strictly controlled within specific limits set by the government.

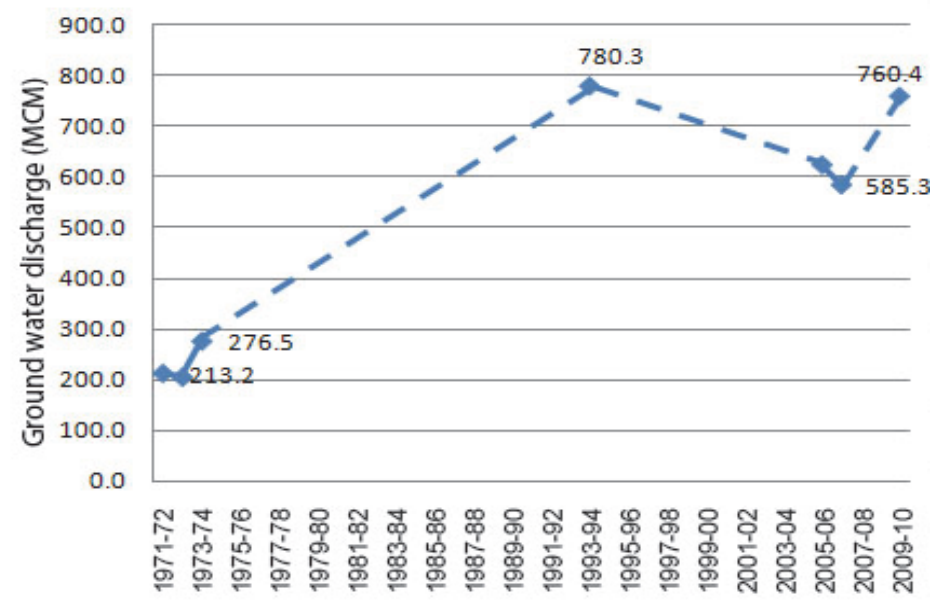

Figure 2.3: Total wells discharge in different sampling years (IWPRI, 2014)

\subsubsection{Change of life style (social pressure)}

Economic development has caused lifestyle changes accompanied with a growing water consumption culture. Although the urban water use accounts for only $3.5 \%$ of water consumption of Rafsanjan region, still the growth of population and water consumption per capita have created problems for a sustainable urban water balance in this region. The average domestic water consumption of Rafsanjan basin in 1994 was 155 litres per day per capita, while by 2007 this number increased to 175 litres per day per capita (RDPI, 2009). Currently the city of Rafsanjan's available urban water is $14 \%$ less than the actual consumption, with a shortfall of -2.2 million m3 (RDPI, 2009). It is one of the highest urban water shortages in the province and it is expected to worsen.

Moreover, the boom of precious pistachio production gave rise to a socioeconomic pressure on the farmers' life styles. The high value of pistachio attracted people and economic activity to the centers of pistachio production, and thereby created a mono-cultural regional economy. Nowadays, in spite of the existence of copper mines and a cotton production tradition in this region, pistachio production has become the first economy and expertise of Rafsanjan. Currently, the majority of Rafsanjan's inhabitants are active in the production, trade and distribution of pistachios (SCI, 2014). Therefore, the mono-cultural regional economy with its high dependency on pistachio production has made this region severely vulnerable to water scarcity. 


\subsection{State}

\subsubsection{Decline of groundwater level (ecological state)}

The rapid rise in the number of pumped wells has led to large scale groundwater withdrawal and consequently depletion of the groundwater level in Rafsanjan. The hydrograph for the Rafsanjan plain in Figure 4 shows a dramatic and continuous drop in the groundwater level, in particular after 1992. During the period 1983-2013, the depth of Rafsanjan plain aquifer has fallen by $20 \mathrm{~m}$. The sharp drop of the groundwater table $(0.75 \mathrm{~m}$ per year) demonstrates vividly the extent of over-use of non-renewable portion of groundwater.

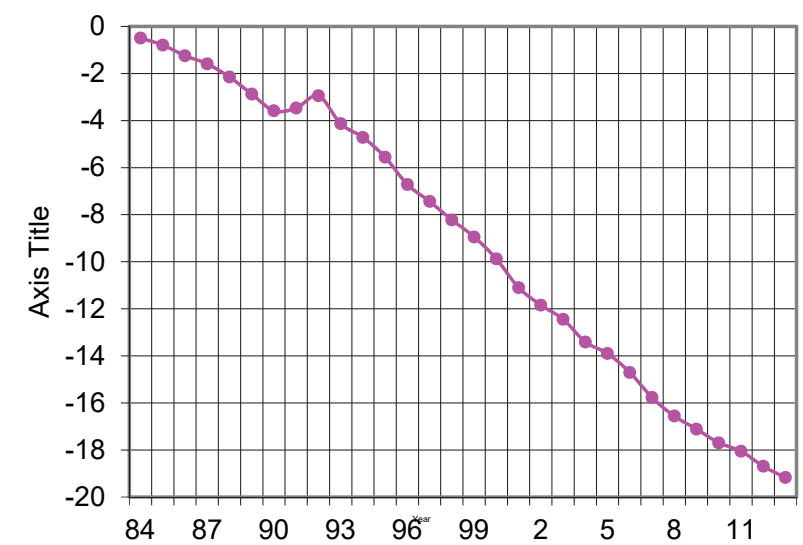

Figure 2.4: Ground water Hydrograph of Rafsanjan Plain from 1983 to 2013. Source: wrs.arm.ir

\subsubsection{Land use change (vegetation area decrease)}

The spatial impact of drought and water scarcity in Rafsanjan can be observed by evaluating the vegetation changes in time series. Here again, the NDVI time series data detect the changes of vegetation caused by drought. The vegetation transformation of the Rafsanjan Township shows major changes in the 28 years between 1986 and 2014 (Figure 5). Although there was widespread expansion of the planted area between 1986 and 2009, around $40 \%$ of these planted areas lost their vegetation after 1998. The level of vegetation in around $30 \%$ of Rafsanjan planted area has decreased. Reduction of vegetation value is because of either change of land use around the four cities (specifically around Rafsanjan city), or drought related decrease of green vegetation. From 2009 to 2014 , there has been only a decrease of vegetation value without any gain, unlike the previous period when both increases and decreases could be observed. 
The change detection map shows that, most of the loss of vegetation occurred around Rafsanjan City. The previous studies of wells' depths and groundwater levels in Rafsanjan Township [13] indicate that the city of Rafsanjan has the lowest groundwater level in the aquifer. Most of the dry wells, with zero or very low discharge rate, are also concentrated around Rafsanjan City. While water accessibility is a critical issue throughout Rafsanjan Township, the impact of agricultural lands drying up seems different in various parts of this region. Some regions like north and east of Rafsanjan city and also the areas around Anar has witnessed more orchards drying up while the orchards between Rafsanjan and Bayaz and also the ones in east of Township appear to be in better condition.

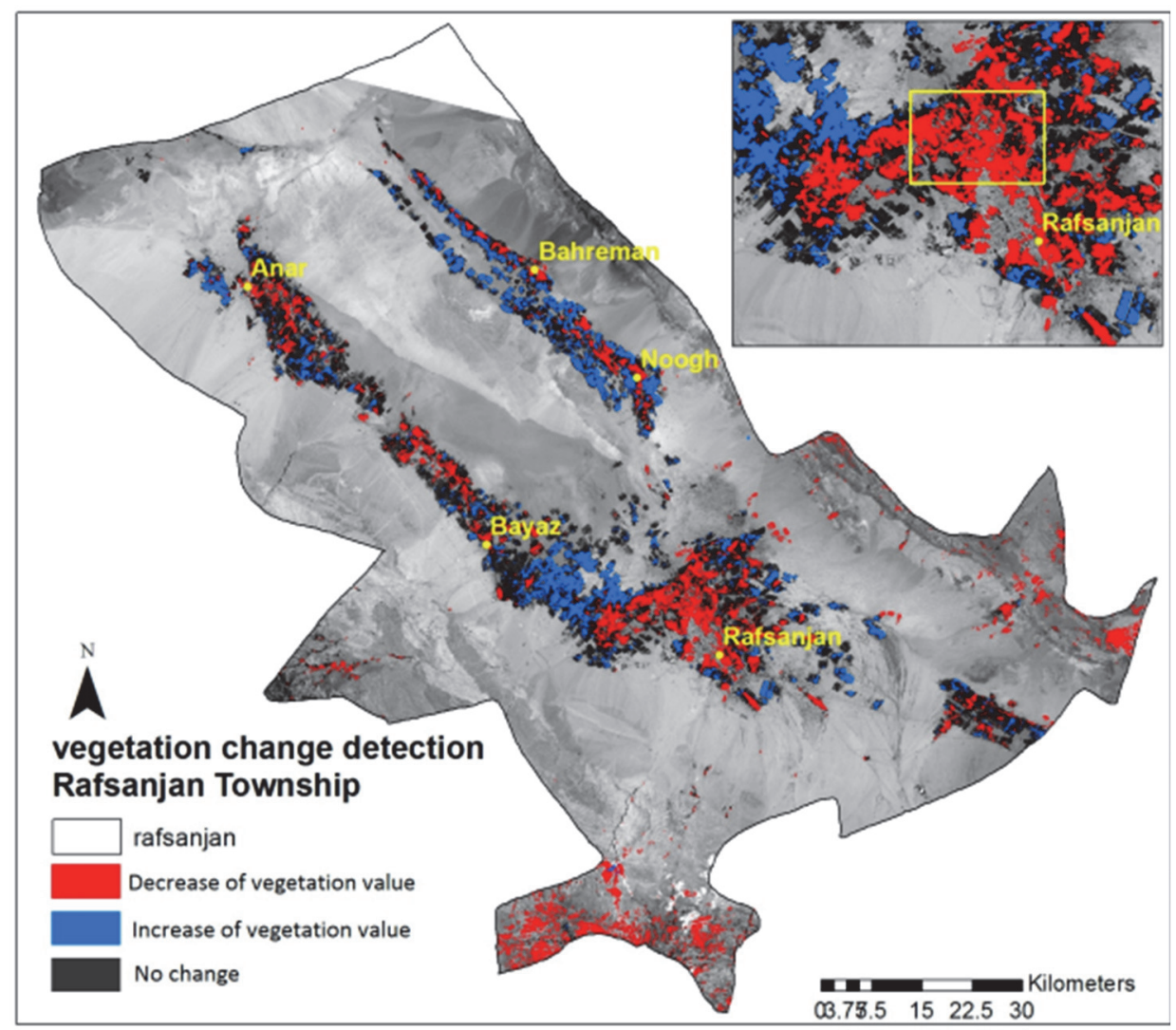

Figure 2.5: Vegetation Change Detection in Rafsanjan Township between 1986 and 2014 (Mehryar et al., 2015)

\subsubsection{Agricultural production and export decrease (economic state)}

Pistachios are Iran's biggest non-petroleum export item and, until 2007, the Islamic Republic was the world's top exporter of this crop. After saffron, 
pistachio is the most expensive agricultural crop in Iran. In $2004,44 \%$ of world pistachio production and $60 \%$ of global exports were from Iran and $16.6 \%$ of the world production belongs to Rafsanjan. In the same year, USA was the second with a production and export share of $13 \%$ and $8.9 \%$ respectively (Razavi, 2005). While the production and export of pistachio has long been a matter of national pride, in 2012 and 2013 Iran has been overtaken as the top pistachio exporter by the United States of America (FAOSTAT, 2015). In the year 2012 the pistachio production of Iran decreased from an annual average of 280 thousand to 150 thousand ton (R.P.P.C, 2014).

\subsection{Impacts}

\subsubsection{Critical ecology of Rafsanjan plain (ecological impact)}

Severe ground water depletion in Rafsanjan has caused malfunction of the wells. Most well throughputs are less than their licence limits which implies that the permissible discharge level of the Rafsanjan plain exceeds the availability of ground water. In some areas, the deepest wells with $400 \mathrm{~m}$ depth do not have access to ground water as mentioned in reports (IWPRI, 2014). Moreover, the large scale ground water depletion resulted in increased salinity and a mix of fresh water with saline water. If the current trend of ground water depletion in Rafsanjan continues further conversion of the pistachio orchards into desert can be expected. Desertification is the consequent impact in case of a business as usual scenario which is considered an irreversible situation socially and economically (RDPI, 2009).

\subsubsection{Land subsidence (environmental impact)}

Excessive groundwater withdrawal in Rafsanjan has also led to new hydrostress of land subsidence in the region (Dehghani et al., 2014, Rahnama and Moafi, 2009, Motagh et al., 2008, Solaimani and Mortazavi, 2008, Gohari et al., 2013). The first acute land subsidence in Iran was observed in Rafsanjan since 1980. The 20 meter depletion in ground water level in the past 20 years is associated with a rate of subsidence of about $5-15 \mathrm{~cm}$ each year (Dehghani et al., 2014). Land subsidence leads to fissures, increase of groundwater salinity, well damages (e.g. well casings rise above the surface) and cracks in buildings and roads (Rahnama and Moafi, 2009). The depth of measured fissures in Rafsanjan plain is up to six meters. Comparing InSAR-derived subsidence maps (Motagh et al., 2008) and satellite images reveals that many of the agricultural and settlement areas are adjacent to dramatically shrinking ground water resources, where land subsidence is very probable. Most of land subsidence of $6-11 \mathrm{~m}$, likewise, occurs in areas with wells of $145-380 \mathrm{~m}$ depth (Figure 6). 

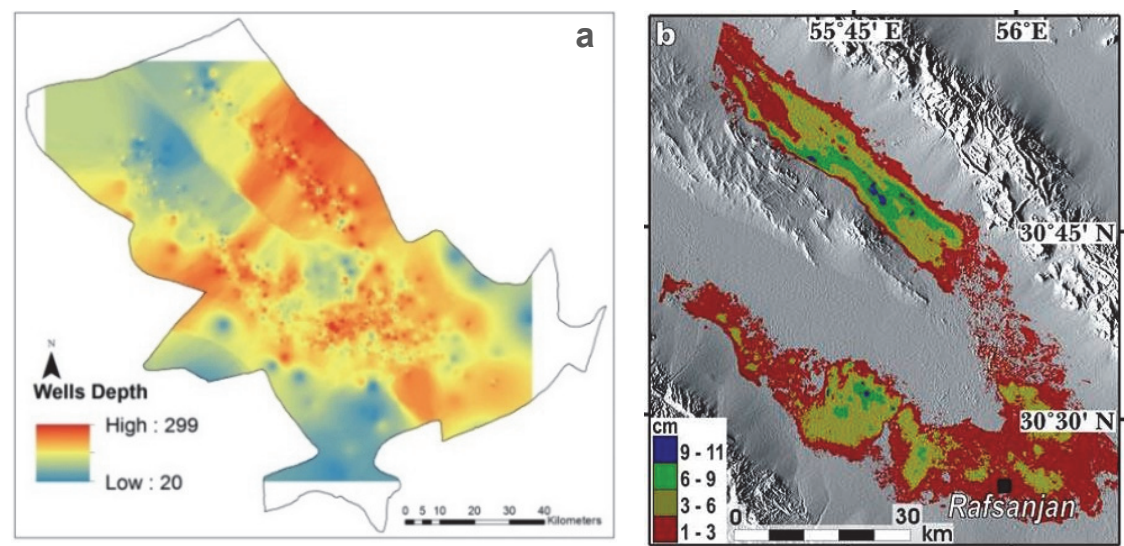

Figure 2.6: a. Wells Depth b. Ground displacement in areas of major land subsidence (Motagh et al., 2008)

\subsubsection{Vulnerable farmers and economy of the region (socio- economic impact)}

The mono-culture economy of Rafsanjan has made the individual and regional economy of this township highly vulnerable to water scarcity. Water crises have inflicted costly adaptive activities of farmers like well deepening, expensive wells repairing, relocation of wells and purchasing of water from other wells. In some parts of Rafsanjan a new unofficial water market is emerging in which well water is traded amongst farmers at very expensive rates (IWPRI, 2014). Adding to the diminishing production returns, farming activity in Rafsanjan is losing its economic profitability. Many farmers, especially the smallholders, have found their well water trade more beneficial than using water for agricultural production. Poverty and immigration are the social and economic impacts of water scarcity which is severely threatening Rafsanjan region (IWPRI, 2014).

\subsection{Responses}

The main responses of government to water scarcity in Rafsanjan can be explained in two categories:

\subsubsection{Supply-oriented management}

Large scale water transfer projects are a supply-oriented management strategy prescribed by Iranian government in Rafsanjan and other basins confronting water scarcity. Currently the inter-basin water transfer projects from adjacent basins and also from the Persian Gulf are among the main responsive policies to protect agriculture of the region against acute water scarcity. Aside inter-basin conflicts over water and the high cost of water transfer projects, previous experiences have shown that these projects actually further stimulate demand and water shortages in the region (Madani, 2014). 
Gohari et al (2013) identifies the water transfer projects as "a fix that backfire", in which side effects of further development and population growth result in higher water consumption, although they solve the problem temporarily. Therefore, supplying more water to the basin without considering the main driving forces of water shortage would only temporarily address the symptoms of the problem and not deal with the fundamentally structural issues that should be addressed.

\subsubsection{Demand-oriented management}

Groundwater pricing policies, well exploitation control, irrigation systems modification, and decreasing the agriculture lands based on the water availability of the regions are among the main responses of the government to lessen the water demand in Rafsanjan. Due to the free access to groundwater the major costs in well water withdrawal are the energy costs to pump water which is reasonably cheap in Iran. Hence, there is no incentive for the farmers to reduce their agricultural water use or optimize their irrigation system. As an example only less than $5 \%$ of the farmed area in Rafsanjan is under pressurised irrigation (IWPRI, 2014), despite the efficiency gains it offers in reduced water consumption per hectare.

Water pricing in Rafsanjan, and generally in Iran, has been suggested and is under development but not yet implemented. In recent years, government has slightly raised energy prices (Madani, 2014) and has also begun to install smart groundwater monitoring devices (Moazedi et al., 2011) to control energy and water use. Nevertheless the effectiveness of these actions is not yet determined. To implement water and energy pricing, all the wells need to be registered and metered to record water use. To be effective and equitable, such measures need to be applied to all wells, both legal and illegal and the latter should also be either registered or shutdown. Water and energy pricing is a general and responsive alternative to the driving force of weak groundwater management, and is aiming to provide incentives to save water consumption although it might also impose a certain extend of income losses to farmers (Varela-Ortega et al., 1998) which may create public dissatisfaction and anger. Overall, more efficient irrigation systems, controlling the mechanisms of groundwater withdrawal and projects to transfer water seem effective solutions for short term, but their long term social and ecological impacts are unknown for policymakers.

\subsection{Conclusion}

The above historical analysis of water-related challenges in Rafsanjan reveals that the seven-year drought considered and perceived by experts and local farmers, is not the only reason of water crisis in this region. A rapid but perhaps ill-conceived economic development accelerated by the weak groundwater 
governance has also had a most destructive impact on water resources. Iran's development plans for this region have mostly focused on economic development, self-sufficiency and self-reliance regardless the potential longterm environmental costs. Besides, the historical analysis of water management and governance in Rafsanjan reveals an increasing exclusion of water users from water governance. This has been driven by nationalization of water and later reinforced by inadequate laws and their unequal enforcement. Unlike the successful collective water management of the traditional Qanat system, the new "Water as a common pool" strategy has reduced peoples' participation and their role in water resource management, which has led to the current groundwater over-exploitation.

While until very recently the government ignored the water crisis the current undesirable socio-ecological state of Rafsanjan shows the symptoms of former approaches resulting in severe water scarcity. Studying the current state of groundwater balance indicates that Rafsanjan has already consumed most of its groundwater supplies. Due to the recent aquifer damage through subsidence, even if there would be a return to the past precipitation levels, the negative impact is irreversible. Satellite image analysis indicates that around 30 percent of the approximately 110,000 ha of pistachio lands in Rafsanjan has lost their vegetation. Moreover, local farmers' perceptions are that 50 percent of pistachio lands has lost its productivity.

Migration and desertification are the current and future impacts of the water crisis. Based on Iranian standards, Rafsanjan plain is earmarked as a "critical prohibited plain" meaning that stabilization of the aquifer's decline is the main goal. Moreover, local farmers are losing their incentive of keeping their pistachio lands. Massive transfers of pistachio lands to other plains is now occurring but this will likely export the water crisis to other regions too.

The inter-basin water transfer projects to pistachio farms is one government policy to respond to water crisis and pistachio production problem. Aside the high costs of water transfer, this action needs careful consideration given the threat of inter basin conflicts as well as an expected further expansion of agricultural land in the region. The other water use control policies and actions from government like water pricing, wells control and irrigation system modification may not effectively respond to the problem if they are not considering effective participation of all stakeholders in water conservation practices. Moreover, a "one size fits all" solution to the water crisis is not possible. The spatial differences of water accessibility and quality, and their various socio-ecological impacts in Rafsanjan requires different but interrelated approaches in decision-making. 
The current analysis based on the DPSIR framework provides a holistic environmental overview over Rafsanjan's water crisis based on literature, government reports and quantitative analysis. However, further studies are required to explore the different stakeholder's perceptions and experiences of the water crisis in various parts of Rafsanjan and the impact of current policies on water use and accessibility. Future scenarios and decision making in Rafsanjan need to consider the involvement of both water users and policy makers at different levels and the socio-economic and ecological differences in various parts of Rafsanjan to enable a sustainable perspective for this very complex socio-ecological problem. 
Chapter 3*

\section{A Structured Participatory Method to Support Policy Option Analysis in Social-Ecological Systems}

* This Chapter is based on:

Mehryar, S., Sliuzas, R., Sharifi, A., Reckien, D. and van Maarseveen, M., 2017. A

structured participatory method to support policy option analysis in a social-ecological system. Journal of environmental management, 197, pp.360-372. 


\begin{abstract}
In this study we demonstrate how to support policy option analysis for a problematic Social-Ecological System (SES) with the help of stakeholder participation. SES sustainability problems 1 ) are highly complex, 2) may lack reliable data, 3) encompass conflicting interests and 4) may require contradictory management interventions. Our approach uses a structured participatory method combining the Driver-Pressure-State-Impact-Response (DPSIR) model together with Fuzzy Cognitive Mapping (FCM) to capture the complexity of the system and simplify its representation for simulation and policy option analysis. Using this novel mixed-method was useful in dealing with above-mentioned characteristics of the complex SES problems. The method was applied in a case study of water scarcity in Rafsanjan, Iran. FCMs were produced for 60 individual farmers and 40 individual researchers and policy makers. Our mixed-method analysis reveals similarities and differences of stakeholder knowledge and problem perception, and simulates the impacts of alternative policy options according to each group's perception. The final result of our case study indicates that farmers in Rafsanjan strongly believe in the impact of economic diversification on reducing water shortage. Yet, they have a low level of trust in the ability of the government to regulate and control water usage, whereas the policy makers and researchers still believe in the role of government control and monitoring policies to deal with water scarcity in Rafsanjan.
\end{abstract}

Keywords: Fuzzy Cognitive Mapping, Driver-Pressure-State-Impact-Response, Social-ecological system, Rafsanjan, water scarcity. 


\subsection{Introduction}

There is a general insight that decision making in complex environmental problems requires an integrated consideration of both social and ecological systems and their interactions: the Social-Ecological Systems (SESs) (Berkes et al., 2000, Folke, 2006, Ostrom, 2007, Ostrom, 2009, Binder et al., 2013). SESs are dynamic systems for which states are constantly changing through the interactions among social and ecological factors (Berkes et al., 2000, Holling and Gunderson, 2002). Due to the dynamic and complex nature of such systems, the environmental management of SES has to deal with four main characteristics: 1) complexity, multi-variability and multi-disciplinarily, 2) illformulation of problems because of lack of or conflicting knowledge, 3) the large number of stakeholders with conflicting values and different views about problems and solutions, and 4) large number of management options and unanticipated consequences of each intervention for the whole system (Xiang, 2013, Olazabal and Reckien, 2015, Vasslides and Jensen, 2016). These four characteristics of SES problems make it difficult for policy makers to use standard dynamic modelling methods to represent and analyse such problems.

The aim of this study is to introduce a structured participatory method to support policy option analysis for a complex SES problem with the help of stakeholder perception. For this objective, we use a combination of a Problem Structuring Method, i.e. Driver-Pressure-State-Impact-Response (DPSIR), with a participatory modelling method, Fuzzy Cognitive Mapping (FCM). The advantages of mixing these two methods is two-fold: methodological and structural. In methodology, DPSIR provides a structuring framework for a complex, multi-variable problem and FCM aids to 1) collect data via experts in data-scarce or poor data environments, 2) involve different interests of stakeholders and 3) simulate the impact of different interventions to the entire system (Reckien et al., 2013, Reckien, 2014, Singh and Chudasama, 2017). Therefore, the mix-method of DPSIR and FCM provides a modelling platform covering all the characteristics of SES problems mentioned earlier: complexity, multi-stakeholder and conflicting interests, data scarcity and unintended consequences of interventions. The structural advantage of DPSIR-FCM method is the use of DPSIR framework for condensation and simplification of multi FCMs outcomes, which will be explained further in section 0 . The final point of our objective is using the stakeholders' perception for modelling the complex SES problems. In our vision, successful policy-making should consider multiple stakeholder views and their perceptions and reactions to policies. Therefore, policy makers need to be able to assess the local knowledge and perceptions of different stakeholders, which might be missed, or be in conflict with official data in a SES problem. 


\subsubsection{The Driver-Pressure-State-Impact-Response (DPSIR)}

DPSIR, adopted by the European Environment Agency (EEA, 1999), is widely used as a problem structuring method to capture and structure the complex causal interactions of human-environmental systems (Bell, 2012, Gregory et al., 2013). This framework categorizes the complex indicators of an environmental problem into social-ecological driver indicators that exert pressures on the system and consequently affect the state of the environmental problem indicators, leading to impacts on the system and triggering societal responses that may in turn feed back to the drivers, pressures, state, or impact variables. Although DPSIR can help to structure and analyse complex SESs problems, it cannot capture trends of change in a dynamic system, as it builds a snapshot of the current situation of a system in the form of causal chains but not its causal networks (Svarstad et al., 2008, Maxim et al., 2009, Atkins et al., 2011, Gregory et al., 2013, Gari et al., 2015). Thus, DPSIR itself does not have the capacity of modelling a complex SES, while its combination with other tools such as FCM can create more valuable outcomes to overcome DPSIR's limitations (Bell, 2012, Lewison et al., 2016).

\subsubsection{Fuzzy cognitive mapping}

FCM is a participatory modelling method recently used in very different disciplines including SES modelling (Fairweather, 2010, Wildenberg et al., 2014, Gray et al., 2015). Structurally, it is a directed graph with feedback, consisting of nodes and weighted interconnections. It is a useful method in eliciting data from experts and stakeholders in data-scarce or poor data cases (Reckien, 2014, Reckien, 2016). It helps to capture stakeholders' perceptions and communicate their knowledge in decision making processes (Papageorgiou and Kontogianni, 2012), and, moreover, by representing the semi-quantitative cause-effect relationships of a system, it can simulate the unanticipated impact of an intervention on different components of a complex SES. However, combining a large number of cognitive maps can result in very complicated maps with too many nodes and connections, making the understanding and analysing of the maps very difficult. According to graph theory, an effective way to better understand the structure of complex FCMs is to condense them. However, the process of condensation is not well-documented nor standardized (Gray et al., 2014). By combining the structuring capabilities of DPSIR with the analysis and simulation capacity of FCMs we demonstrate a potentially fruitful method for FCM condensation.

\subsubsection{Case study}

We used our methodology in the case of water scarcity in Rafsanjan, Iran; a major producer and exporter of pistachios in Iran. Being in an arid and semiarid region, pistachio farmers in Rafsanjan are dependent on ground water for orchard irrigation. Rapid agro-economic development of the land and 
unsustainable water management have led to a high water demand of pistachio lands and overexploitation of groundwater. Frequent droughts and climate change are also contributing to the depletion of the area's aquifer.

Water scarcity in Rafsanjan is a tightly intertwined social-ecological problem. Water is almost free of charge, the energy for pumping groundwater is subsidised and the government has shown little control on the growing number of wells and their extraction limits. While pistachio farmers' associations have co-operated in the management of shared wells, they are not generally involved in policy and decision making about water management. Pistachio associations also have little contribution in water demand reduction activities since their main concern is the increase of production and export capacity of pistachio. This situation indicates a complex SES where various variables from social, economic, ecological and political disciplines are interdependently affecting the state of ground water in Rafsanjan.

Lack of data and their unreliability are challenges too. Besides conflicting data from different reports, government data are inaccessible due to the high sensitivity of the water crisis in Iran and its political and societal implications. Currently, there is an "every man for himself " situation in Rafsanjan: farmers maximize water extraction for their remaining lands or move the pistachio orchards to other regions once they can no longer make the desired profit in Rafsanjan. Attempts to manage groundwater reserves at a sustainable level are not adopted. There are many different actors and institutions with conflicting interests that complicate the decision making process related to groundwater and pistachio production in Rafsanjan.

There are alternative strategies for monitoring and limiting water use: applying advanced irrigation technologies, regulating water supply and demand provisions, and educating farmers, but their effectiveness in this complex SES of Rafsanjan is unclear. Most of these strategies are either new or have not yet been applied. Even when some water is saved, it is often used for the expansion of pistachio cultivation (Mehryar et al., 2015, Mehryar et al., 2016). Considering the sustainability challenges of water scarcity in Rafsanjan, a tool to help policy makers in assessing the impact of their policy options is potentially useful. The tool should be able to deal with complexity of various changing factors, conflicting interests of stakeholders, the lack of reliable data and the unanticipated consequences of the policy interventions in the whole system. 


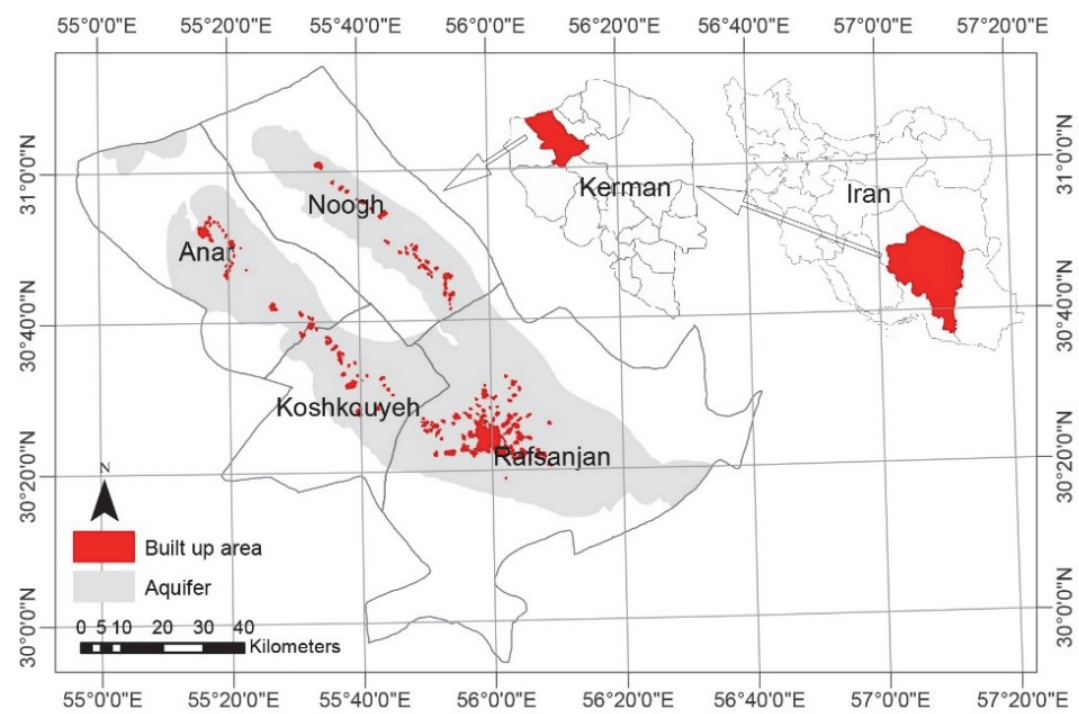

Figure 3.1: Rafsanjan Township in Kerman province and in Iran

\subsection{The methodological framework}

The methodology is designed to support policy options analysis in a multiinterest system with poor data or conflicting knowledge about system behaviour among the stakeholders and scientific studies. This methodology comprises four steps.

Step 1: Provide a preliminary problem structuring DPSIR framework of the SES problem indicators based on literature and statistical data to set objectives and system boundaries.

Step 2: Create different stakeholders' FCMs, which together present the collective knowledge of participants helping to define indicators of the system and their relations when there are not enough reliable objective data.

Step 3: Use the DPSIR framework (step 1) to condense and structure the set of FCMs (step 2) through an iterative adjustment process producing a perception model for each stakeholder group.

Step 4: Simulate with the perception models the impact of different policy alternatives and assess their effectiveness on the state of system.

Each step is described in more detail below.

\subsubsection{Step 1: Project objectives and system boundaries via DPSIR}

To model the most important indicators of a complex SES it is important to define the exact problem and the related project objectives (Jetter and Kok, 2014). Otherwise, there is a danger that the model will be too general to be analysed (Mourhir et al., 2016). To do this, we created an initial DPSIR framework to structure the problem components based on scientific studies, 
reports and statistical data which are mostly used in environmental decision making. The advantage of using an initial DPSIR model is threefold: 1) it helps to focus on particular information and data needed to be derived from participants later and also identifies useful questions for appropriate knowledge elicitation; 2) the modeller's initial understanding of the problem domain can serve as a stimulus in respondent surveys in a later stage (Jetter and Kok, 2014), although it should not be imposed in the process of stakeholder knowledge elicitation (step 2);3) the content of this step is useful to check stakeholder inputs versus accepted scientific facts (Jetter and Kok, 2014).

\subsubsection{Step 2: Stakeholders knowledge modelling via FCM}

In this step FCM was applied to model the perceptions and knowledge of stakeholders about problem indicators and their relationships. A participatory elicitation process ensured that all important variables of a system, in spite of lack of measurable quantitative data, were captured, as well as the different and sometimes conflicting interests of stakeholders to bring about a joint decision. (Malena, 2004, Buruzs et al., 2015, McKenzie, 2005).

FCM, by its structure, is a fusion of a neural network and fuzzy logic (Carvalho, 2013). It contains a signed and directed graph with feedback, consisting of nodes and weighted interconnections (Kosko, 1993). Each node represents a concept of the system which can be either a tangible object such as 'resource quantity' and 'quality', or an abstract object like 'public trust' (Vasslides and Jensen, 2016). The weighted connections represent the causal relationships between concepts. The individual or group participants identify the important concepts and then link them with weighted and directed arrows.

To develop the FCMs, we used the individual face-to-face interview technique (Gray et al., 2014). FCM data collection was done by adapting the suggestions of (Özesmi and Özesmi, 2004), Jetter and Kok (2014) and Gray et al. (2014), as explained in following sections.

\section{Stakeholder groups}

The first step was to determine the right groups of stakeholders. The main aim of involving different stakeholders in this methodology was to gather various kinds of knowledge and views about the problem domain. Different stakeholders were categorized based on their knowledge and experience of the system. For example, there are many stakeholders in Rafsanjan involved in causes and impacts of water scarcity, including policy makers for water, agriculture and environmental sectors, NGOs, researchers, and farmers. While doing the interviews we learnt that both the policy makers and researchers in Rafsanjan have similar perception of the system problem, probably because both of them have their knowledge based on official studies and scientific data while the farmers have the local knowledge based on their own experience. 
Consequently, we formed two groups: policy makers and researchers (P\&R), and farmers to address the most conflicting perceptions. More information about the difference of group and individual interviews in this case study as well as defining the sample size is explained in appendix $1 \& 2$.

\section{Interviews and questions}

In order to keep the variety of voices and reduce "elite bias", all the mind map production during the interviews were done without any predetermination of concepts from preliminary step of research. Rather, the interviews were conducted with open-ended and free style questions where the interviewees were free to choose their own concepts. However, the questions were following the predetermined categories of DPSIR framework (table 3.1).

Table 3.1: Interview questions and DPSIR categories that each question aims to find concepts related to them.

\begin{tabular}{|l|c|}
\multicolumn{1}{|c|}{ Interview questions } & $\begin{array}{c}\text { Related DPSIR } \\
\text { category }\end{array}$ \\
\hline $\begin{array}{l}\text { 1. How is the current water situation in Rafsanjan (your } \\
\text { specific region for farmers)? }\end{array}$ & Sate \\
\hline $\begin{array}{l}\text { 2. What do you think has influenced/caused the current } \\
\text { water situation in the region? }\end{array}$ & Driver \& Pressure \\
\hline $\begin{array}{l}\text { 3. What have been the impacts of the new water situation } \\
\text { in the region? }\end{array}$ & \multicolumn{1}{|c|}{ Impact } \\
\hline $\begin{array}{l}\text { 4. What have been the adaptive actions to this new } \\
\text { situation? }\end{array}$ & Response \\
\hline
\end{tabular}

Defining the concepts by stakeholders helped us to capture most possible aspects of the SES problem. This method of collecting data is useful specifically in such cases where the problem itself is ill-formulated. Yet, having a preliminary understanding of the research area through DPSIR framework increased the theoretical sensitivity of the interviewer and supported the focus and scope of our interviews.

Talking about the questions required long discussions lasting from 30 minutes to three hours per interview. During the discussions, the networks were drawn on paper by the interviewer who regularly validated these with interviewees. The concepts and the directed links between these were drawn at the same time to establish causal connections. On several occasions during the interviews, we needed to specify the exact time scale for the questions. It has to be noted here that FCM is suitable for short-term time series analysis and prediction (Buruzs et al., 2015). FCM by its nature is not capturing the episodic events or temporal sequence of a system. It only represents the recently experienced events that have caused the current state. Therefore, the exact period for each question should be identified for the variables with different sequences of state. For example, when we are talking about the variable "agriculture land area", there is a period of expansion because of agroeconomic growth in Rafsanjan followed by a period of shrinking because of 
water scarcity. Thus, we asked participants about their latest experiences of agriculture land area change.

\section{Weighing the connection linguistically and numerically}

After defining the whole network, the interviewees were asked to weigh the connections to show the degree of influence of concepts on each other. It was important to explain that the connections reflect causal relations among variables meaning the increase or decrease of one variable causes the increase or decrease of another variable (Carvalho, 2013).

Causal weight can be either a number in the interval $[-1,1]$, or a linguistic value such as "very low", "low", "average", "high" and "very high". In a later stage, these linguistic values can be translated into numerical values in the range of $[-1 ; 1]$. During the interviews we noted that some people, especially farmers, provide a linguistic judgment (Abdullah and Khadiah, 2011), while policy makers and researchers tend to give a scale number. Therefore, a five-point numerical of linguistic scale was shown to the respondents and during the weighing, they used the one that was most understandable to them.

\subsubsection{Step 3: FCM post-processing using DPSIR framework}

After creating all individual FCMs, each map was transformed into an adjacency matrix. For each map all the concepts were listed in the horizontal and vertical axis of a square matrix and the weights of connections are placed in the intersecting cell of the respective concept, which is a value between -1 and 1 (Carvalho, 2013, Olazabal and Reckien, 2015). Before transferring maps into the matrices, the different wording concepts in individual maps should be converted into a common terminology or code (Reckien, 2014). It means the variables with similar meaning are coded with one concept name, e.g. 'dry climate' and 'lack of precipitation'. To avoid a biased terminology coding, we went back to the respondents to check the validity of the standardized concept names.

\section{Network aggregation}

All individual cognitive maps can be mathematically augmented using matrix addition to create a social (i.e. multi-stakeholder) cognitive map (Özesmi and Özesmi, 2004). The entries of the new augmented matrix are the average of the connection weights assigned by individuals. Aggregating the individual maps was done very carefully. In addition to using standardized terms, sometimes concepts and dis-concepts were used in different maps (e.g. optimal farming and non-optimal farming). In such cases one of them was modified and the sign of any relationships was also reversed to maintain effect consistency. 


\section{Quantitative and qualitative condensation}

Aggregated maps contain more concepts and connections than any of the individual maps. Since maps with more than 20-30 variables look overcomplicated for gaining insights (Özesmi and Özesmi, 2004), condensation is used to simplify the complex cognitive maps. Two types of condensation were used in our case: quantitative and qualitative condensation. In quantitative condensation the concepts with the least influence in the system, i.e. the nodes with a centrality number less than 1 , have been eliminated. In qualitative condensation, the variables were combined and nested into the upper level encompassing categories. Each new larger subgroup consists of a group of variables connected to other variables outside the subgroup. The new connections' weights of the subgroup is the average of all connection weights of variables inside the subgroup. But the connections inside the subgroup have not been kept, following the accepted argument that causality is not selfreflexive, i.e. a concept cannot cause itself (Carvalho, 2013).

In FCM technical studies, the process of defining the main subgraph themes is not yet clear. In some applications, subgroup themes emerge with the help of participants, which is very difficult and confusing with individual-FCMs. In other studies, the subgroups emerge from the relevant larger theoretical framework of the studied issue (Nakamura et al., 1982, Özesmi and Özesmi, 2004, Kontogianni et al., 2012). Here we used the principles of "directed content analysis" method in which the analysis of collected data starts by using prior research findings as guidance for identifying key concepts as initial coding categories (Hsieh and Shannon, 2005). Considering the setting in Rafsanjan the preliminary concepts of DPSIR conceptual model helped us to define the different subgroups of the system. Any concepts of FCM findings that could not be categorized within DPSIR subgroups were given a new subgroup. Moreover, data analysis with prior research guides the discussion of findings which might be contradictory with such preliminary researches or might extent and enrich that (Hsieh and Shannon, 2005). In our case, having the DPSIR model allowed comparison of the literature data captured by the DPSIR model with knowledge-data captured by the FCM model in later steps.

\section{Calibration of the model}

The FCM model approach does not represent the absolute truth of a system, rather it models the stakeholders' perceptions of a system. Therefore, it cannot be verified using quantified or historic data that are accepted as truths (Penn et al., 2013, Jetter and Kok, 2014). Moreover, the summation of all individual perceptions may eventually show different dynamic properties from what an individual may infer from his own knowledge. Therefore, the aggregated social FCM may not necessarily represent the perception of each individual and thus cannot be validated by individual stakeholder members. Following the suggestion of Jetter and Kok (2014) as applied by Olazabal and Pascual (2015), 
we used a simple bivalent nodes cognitive map that expresses the very wellknown dynamics captured from respondents during the interviews. For example, based on farmers' perceptions it was very well understood that an increase in "irrigation system modification" has caused an increase in "pistachio production" and an increase in "groundwater depletion", or an increase in "small holding" has caused an increase in "unregulated ground water use". These three examples were checked and were matched with the basic behaviour of the modelled system.

\subsubsection{Step 4: Assess the impact of different policy options}

Having the current state model of the system perceptions, one could also ask "what-if" questions to understand the impact of different policy options. This simulation shows us 1) the effectiveness of each policy option based on the stakeholders' knowledge of the system and 2) the acceptance of each policy options by two selected groups of stakeholders, farmers and policy makers/researchers.

For running the policy options simulations, the steady state of the model was first calculated. The steady state shows where the system would go if nothing changed based on the stakeholders' view of the system. For calculating the steady state, all the initial values of the concepts were set to 1 , assuming they are all equally active. Then, matrix multiplication yields new values for each concept. These new values are then normalized, using a so-called squashing function, to return to values between 0 and 1 (Reckien et al., 2013). This procedure is repeated until all concepts reach a steady state representing the baseline. Theoretically, resulting values can go into a limited cycle or into a chaotic pattern (Dickerson and Kosko, 1994). In our analysis, all the concepts reached a steady state within 30 iterations.

In the second step the results of a manipulated system, i.e. representing different policy options through the "what-if" analysis, are calculated. It explains where the system (perceived by stakeholders) would go if one or more policy options were to be implemented (Kontogianni et al., 2012). In our study, we separately simulated impacts of four policy options for the case of water scarcity in Rafsanjan. Among many possible policy options we chose the ones that significantly change the influence of one or more of the main drivers in our model, i.e., mono-economy, lack of government control, traditional agriculture/irrigation system and small-holding, using the FCMapper program. The four policy options are explained in Table 3.2. To simulate the implementation of policy options, in each policy option run one or more concepts are clamped as continually high or low (Kosko, 1986b, Özesmi and Özesmi, 2004, Gray et al., 2015) to a value of 0 or 1 (Singh and Chudasama, 2017). If the policy option increases a concept, it is clamped to 1 and if it permanently decreases a concept, it can be clamped to 0 (Reckien, 2014). For 
example in the economic change policy option the two concepts of Monoeconomy and Agriculture area expansion are clamped as permanently low (Table 3.2). The value of clamped concepts remains fixed in each time step of matrix multiplication and the change of other concepts was calculated under the new policy implementation. This was repeated until the unclamped concepts reach a steady state. The simulation process is done by using the FCMapper program.

Table 3.2: Policy options, the clamped concepts and their values in each policy option

\begin{tabular}{|c|c|c|}
\hline Policy option & What-if question & $\begin{array}{c}\text { Clamped concepts } \\
\text { and their clamped } \\
\text { values }\end{array}$ \\
\hline 1) Economic change & $\begin{array}{l}\text { What if the economy of } \\
\text { the region changes from } \\
\text { agriculture to other } \\
\text { sources of economy like } \\
\text { industry and service } \\
\text { production? }\end{array}$ & $\begin{array}{l}\text { Agriculture } \\
\text { expansion }=0 \\
\text { Mono-economy }=0\end{array}$ \\
\hline $\begin{array}{l}\text { 2) } \\
\text { Irrigation/Agriculture } \\
\text { system change }\end{array}$ & $\begin{array}{l}\text { What if the decision } \\
\text { making focuses on } \\
\text { modification of the } \\
\text { irrigation/agriculture } \\
\text { system by using } \\
\text { advanced technology to } \\
\text { improve production? }\end{array}$ & 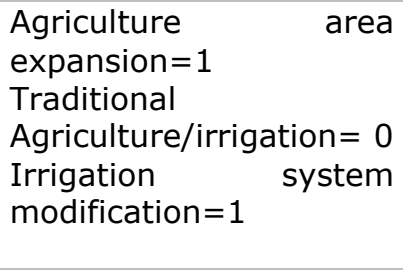 \\
\hline $\begin{array}{l}\text { 3) People } \\
\text { participation and } \\
\text { integrated } \\
\text { landowning }\end{array}$ & $\begin{array}{l}\text { What if the decision } \\
\text { making focuses more on } \\
\text { participatory } \\
\text { management methods? } \\
\text { Integrated landowning } \\
\text { for optimized irrigation } \\
\text { and agriculture is one of } \\
\text { the main solutions that } \\
\text { needs farmers' high } \\
\text { involvement. }\end{array}$ & $\begin{array}{lr}\text { Lack of } & \text { people } \\
\text { participation }=0 & \\
\text { Small holding }=0 & \\
\text { Community } & \text { based } \\
\text { organization }=1 & \end{array}$ \\
\hline $\begin{array}{l}\text { 4) Government } \\
\text { control, monitoring } \\
\text { and limitation }\end{array}$ & $\begin{array}{l}\text { What if the government } \\
\text { implements appropriate } \\
\text { controlling and } \\
\text { monitoring policies? }\end{array}$ & $\begin{array}{l}\text { Subsidy }=0 \\
\text { Lack of government } \\
\text { ctrl=0 }\end{array}$ \\
\hline
\end{tabular}

Lastly, the final values of the concepts in the non-manipulated system (baseline steady state) are compared to the final values of concepts in the adjusted policy scenario. Comparing the results of different policy options to the baseline scenario determines the desirability level of each scenario by two stakeholder groups of farmers and P\&R (section 3.3.4). 


\subsection{Results}

The main research question deals with the likely impact of alternative policy options on water scarcity in the complex, dynamic social-ecological system of Rafsanjan. To answer this question, we developed and applied our structured participatory method to simulate SES behaviour without and with new policy regimes.

\subsubsection{Analytical DPSIR based on literature study and field data}

The output of step 1 of the methodology, i.e. the analytical DPSIR framework of water scarcity in Rafsanjan, has been developed and described in an earlier paper (Mehryar et al., 2016). This framework input is based on spatial datasets, statistical data, scientific studies on quantitative-data and governmental reports which are basically the policy makers' input data. Figure 3.2 represents the causal network generated by the DPSIR framework. More information about each category of this framework can be found in appendix 3 .

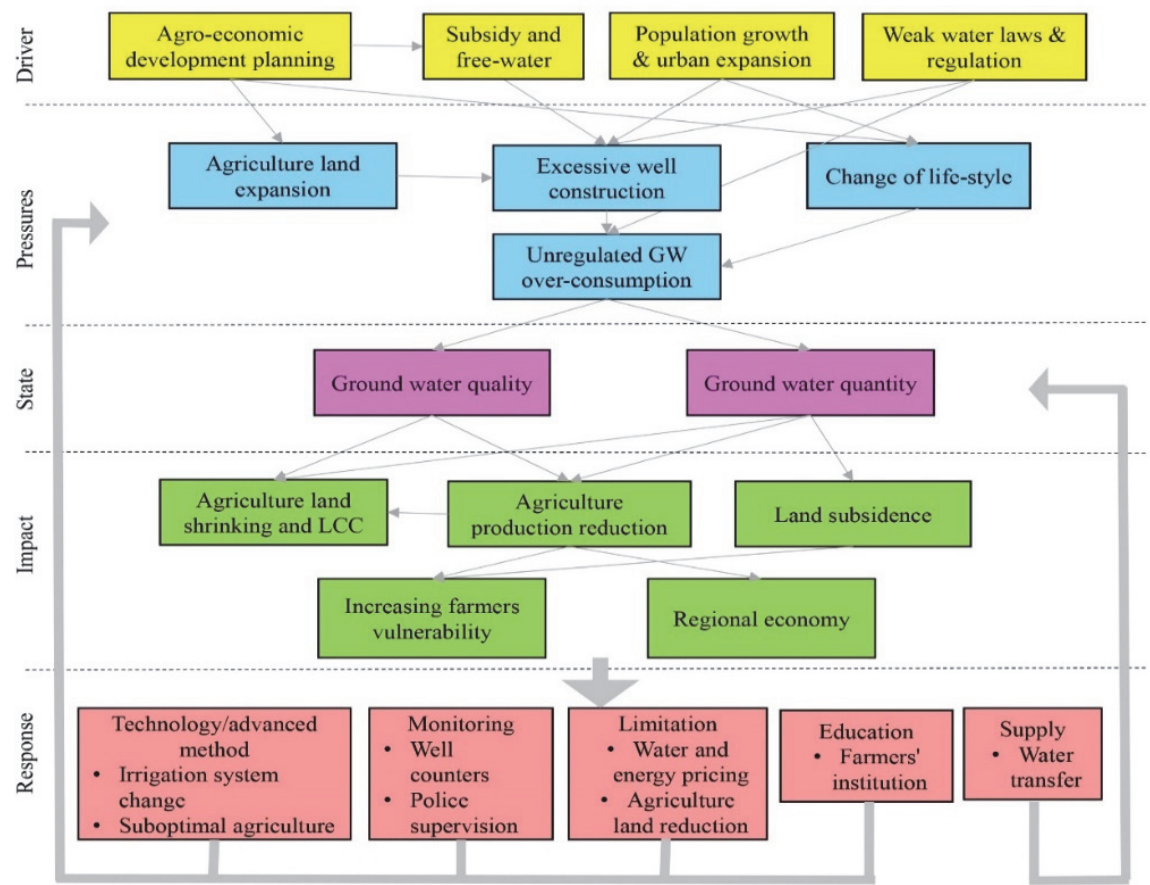

Figure 3.2: Preliminary DPSIR framework of water scarcity in Rafsanjan, based on literature and statistic data. Indicators are categorized in five different layers (driver, pressure, state, impact and response) and the causal relations are shown with directed arrows. The concept and connections have no value or degree. 


\subsubsection{FCM model structured by DPSIR framework}

After generating and aggregating all individual FCMs, the two stakeholders' maps, so-called social FCMs, were composed and condensed through applying the preliminary DPSIR framework (Figure 3.3 and Figure 3.4).

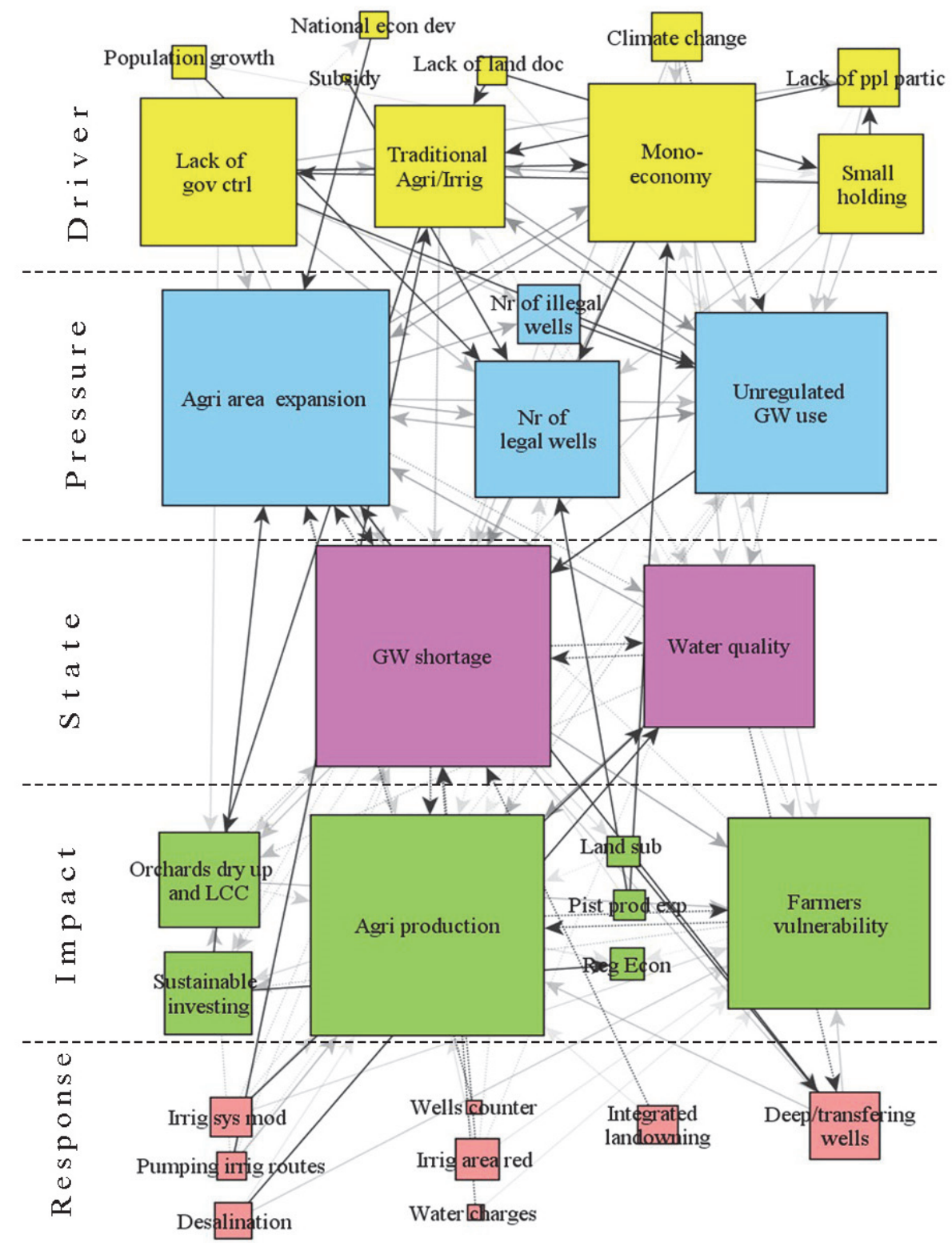

Figure 3.3: Farmers' Social Fuzzy Cognitive Map of current state in DPSIR framework. The size of the nodes refers to the centrality of impact, i.e. the weighted sum of input and output connections. The thickness of the connections shows their weight value. Connections with positive weights are straight; and with negative weights are shown in dashed line. 


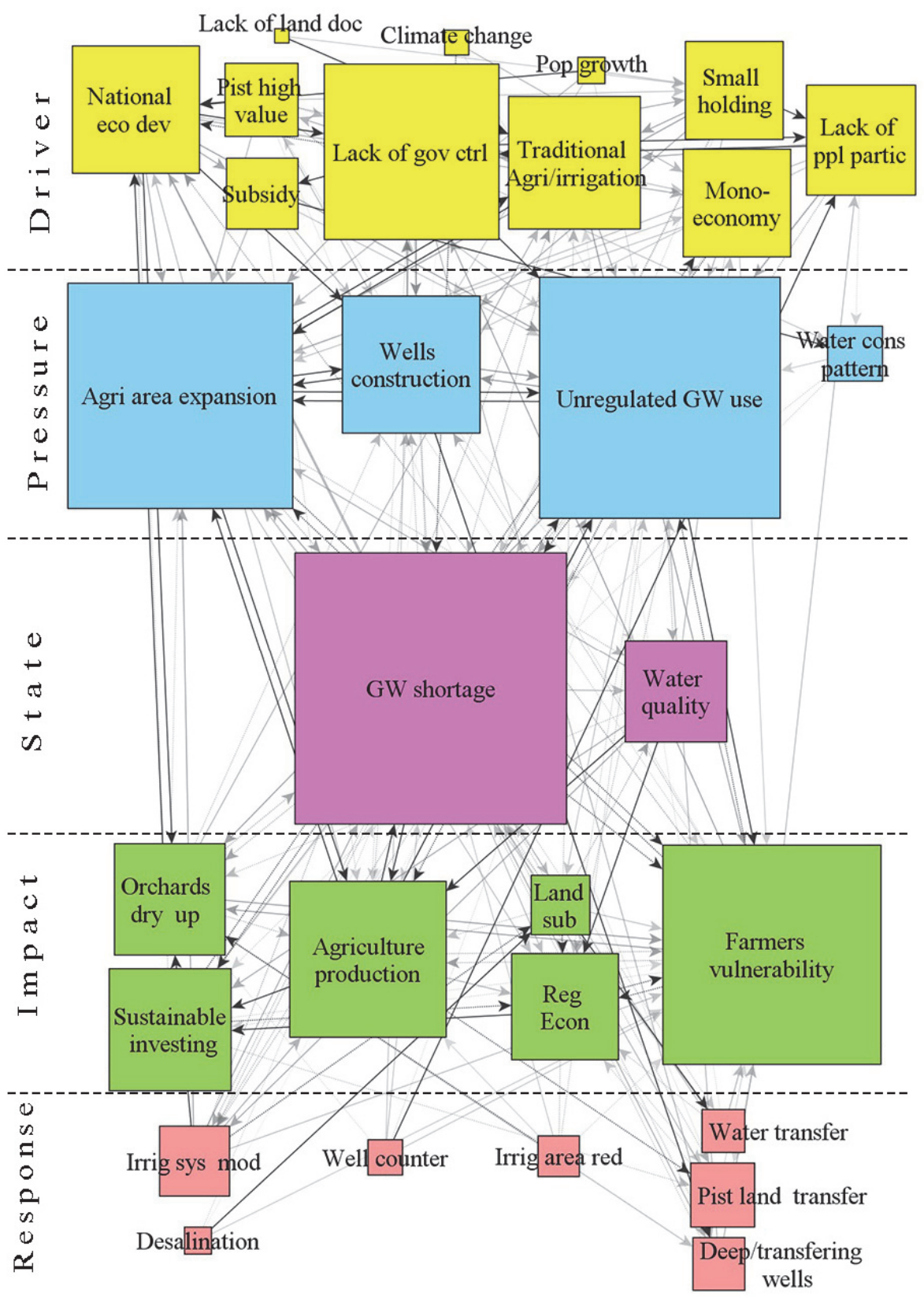

Figure 3.4: Policy makers' and researchers' Social Fuzzy Cognitive Map of current state in DPSIR framework 


\subsubsection{DPSIR-FCM analysis}

Having two different DPSIR-FCM models gives us the possibility to have threefold comparisons: 1) comparing knowledge data versus available literature/statistical data, 2) comparing different stakeholders' perceptions, 3) comparing inter-layers DPSIR categories.

\section{Knowledge data versus literature data}

There are many concepts in stakeholders' FCMs that are not considered by research studies and government reports that are based on quantitative and statistical data mostly. These missing concepts in scientific studies indicate that the problem itself may not be well formulated for policy makers due to a lack of knowledge of the system's complex structure and behavior.

Among the main drivers of water scarcity, smallholding and mono-economy are new concepts mentioned by stakeholders but less studied in researches and reports. Small land holding emerged after the 1963 land reform in Iran. The consequences of reform not only replaced the Qanat collective management system with individual pumping well systems (Mehryar et al., 2015), but also resulted in suboptimal irrigation and wells over-exploitation comparing to the large size lands. A costly new irrigation system for small lands is an obstacle for changing the traditional irrigation system of small holders. On the contrary, climate change and population growth have been mentioned among the less important driving forces in both P\&R and farmers' FCMs whereas these are usually mentioned as the main causes of water scarcity in reports and studies.

In the part of pressures, often the wells construction and growing number of wells are mentioned as the main causes of ground water over-exploitation (Mehryar et al., 2015). Nevertheless, from the stakeholders' perception, the "unregulated wells water consumption" puts a much higher pressure on the system than the "number of wells". According to the farmers' knowledge, the growing number of legal wells was more relevant than the number of illegal wells. Therefore, the problem is not excessive well construction without the governmental permission, but rather the weakness of water authority control and supervision that leads to excessive groundwater exploitation via legal wells. In the relatively chaotic situation after the Islamic Revolution in 1979, corruption and populistic water governance were considered as the main components leading to the weak wells control and supervision.

Although official reports and researches emphasize land subsidence in Rafsanjan Township (Dehghani et al., 2014, Rahnama and Moafi, 2009, Motagh et al., 2008, Solaimani and Mortazavi, 2008), this issue is not perceived as an important impact of water scarcity by farmers. Some farmers have experienced fractures in their buildings and infrastructure (as the symptoms of land 
subsidence) and P\&R know somehow about it from scientific studies but because land subsidence is hidden and slow it is almost ignored, though in the long run the loss of aquifer capacity due to subsidence may reduce the likelihood of aquifer recharge even if precipitation increases.

And finally among the responses derived from the scientific studies and reports, the informal adaptive responses from lay people are not addressed. Here pistachio land transfer, desalination and integrated land owning are among the farming community's responsive actions that are not yet considered. Most of the large land owners are planting pistachio in other regions with better access to water and gradually abandoning their lands in Rafsanjan. Farmers believe this trend is resulting into "water scarcity transfer" to those places where the pistachio land is intensified.

\section{Different stakeholders' perceptions}

An interesting difference between the two stakeholders is that the P\&R group perceives lack of government control as the main driver of water scarcity whereas farmers see the pistachio dependent economy or mono-economy as a more important driver (Figure 3.3 and Figure 3.4). Traditional agriculture/irrigation system is among the most important drivers for both groups. Yet, there are driver variables mentioned by one group but not the other, e.g. the lack of land document recognized by farmers as the main cause of traditional irrigation system. For switching from flood to drip irrigation farmers can get government loans if they have a land title document. However, after the 1963 land reform and the 1979 revolution, many farmers do not have such a document and therefore have less interest in changing their traditional irrigation system. This is an example of a driver that is not perceived as important by the P\&R group. Moreover, the subsidy and free water are drivers only noted by the P\&R group and not by farmers.

Another noteworthy difference is that the P\&R group perceives farmers' vulnerability as the most important (highest centrality) impact of water scarcity, whilst the farmers themselves perceive the decrease in agriculture production as the most important impact, higher than their vulnerability. The much higher in-degree value of the agriculture production concept than its outdegree in both groups (Figure 3.5) indicates that the decrease in pistachio production has not yet influenced other concepts like poverty and migration much. However, both groups mentioned that the visible social-ecological impacts of agriculture production change like immigration, poverty and desertification will be revealed in the very near future.

Considering the response category of variables, the most important responses for P\&R are irrigation system modification and pistachio land transfer, whereas deepening and transferring wells is the most important response from farmers. 


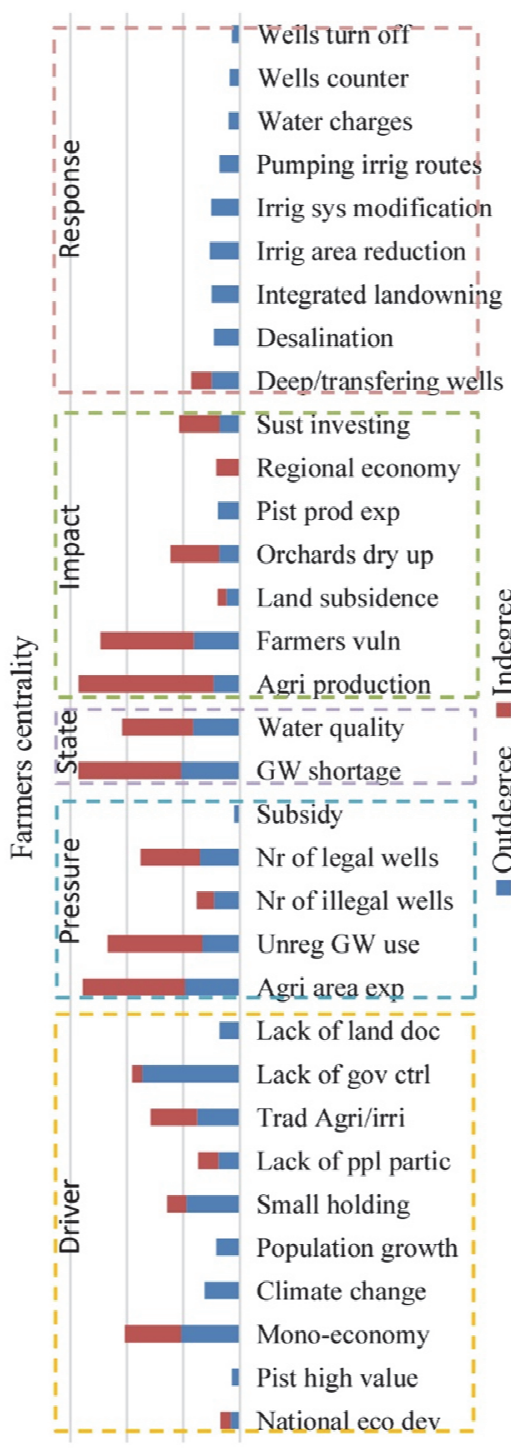

$15 \quad 10 \quad 5 \quad 0$

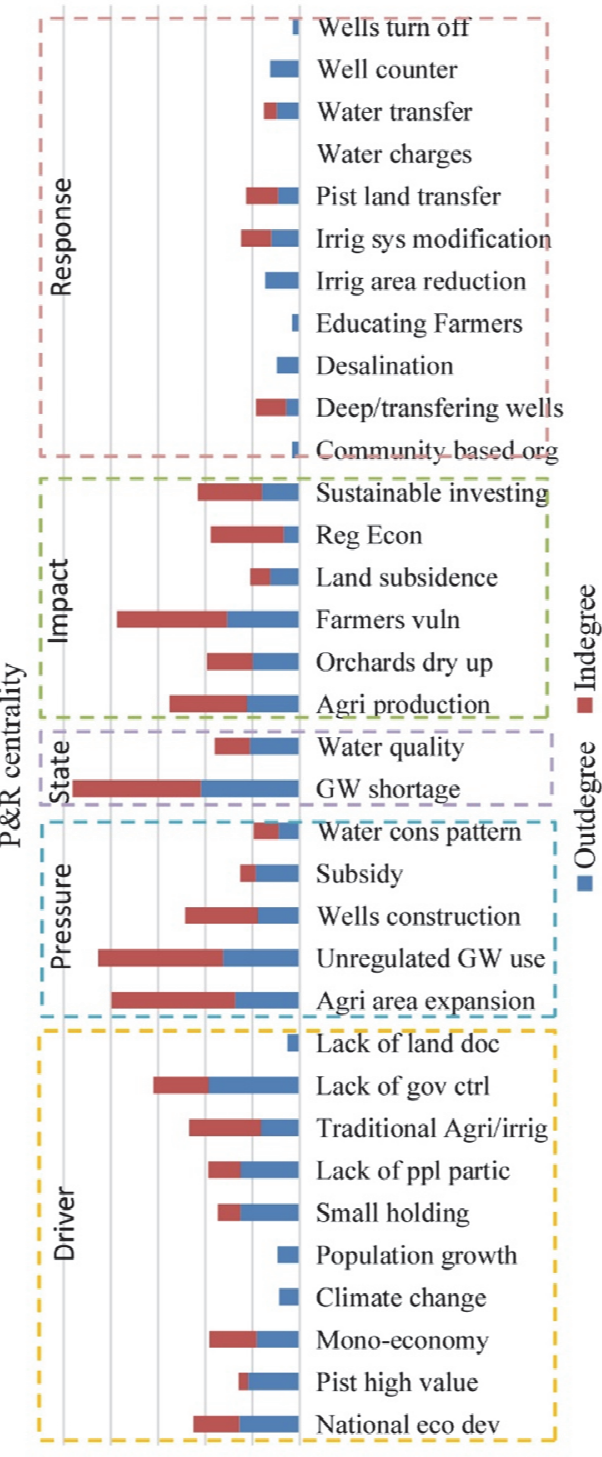

$\begin{array}{llllll}25 & 20 & 15 & 10 & 5 & 0\end{array}$

Figure 3.5: In-degree, Out-degree and Centrality of variables in Farmers and P\&R social map. In-degree and out-degree of each variable indicate the sum of all connections' values entering and exiting that variable respectively. Centrality is the summation of the in-degree and out-degree of a variable. 


\section{Inter-layers DPSIR categories}

We can also compare the structure of FCMs in DPSIR categories via the graph theory to define the category of variables influencing the system more. Based on the graph theory, the variables with higher density are the more important ones, but the variables with higher out-degree than in-degree are the ones that can better initiate a change to the system than receive the change from system dynamics (appendix 5). In both FCMs the important impact and pressure categories have a higher in-degree than out-degree (Table 3.3, Figure 3.5), meaning these are more influenced by system dynamics. So a change in their behaviour is highly related to a change of input they receive from other categories, i.e. driver, state and response categories, whereas all driver and response concepts have a substantial higher out-degree than in-degree, meaning a change in their value may easily and more effectively change the behaviour of the system than other categories. However all the response concepts have very low centrality, which means that they have a relatively low impact on the system as a whole. This situation indicates the low effectiveness of adaptations and solutions made so far.

On the basis of the graph theory analysis of the two FCMs, the higher density of the P\&R social FCM (Table 3.3) indicates that they perceive a higher level of complexity within the system than farmers do. Generally speaking, the $P \& R$ group has a more diverse and broader view about various aspects of the system than farmers, while the farmers' knowledge of the system is limited to fewer variables.

Table 3.3: FCM graph theory analysis per stakeholder group

\begin{tabular}{lllllllllll}
\hline & $\mathbf{N}^{\mathbf{a}}$ & $\mathbf{C}^{\mathbf{b}}$ & $\mathbf{T}^{\mathbf{c}}$ & $\mathbf{R}^{\mathbf{d}}$ & $\mathbf{D}^{\mathbf{e}}$ & $\mathbf{I D}^{\mathbf{f}}$ & $\mathbf{O D}^{\mathbf{g}}$ & $\mathbf{I P}^{\mathbf{h}}$ & $\mathbf{O P}^{\mathbf{i}}$ & $\mathbf{I S}^{\mathbf{j}}$ \\
\hline Farmers & 33 & 124 & 14 & 1 & 0.11 & 1.44 & 3.28 & 4.85 & 2.89 & 7.67 \\
P\&R & 34 & 209 & 10 & 0 & 0.18 & 3.1 & 4.85 & 7.66 & 5.26 & 8.67 \\
\hline & $\mathbf{O S}^{\mathbf{k}}$ & $\mathbf{I I}^{\mathbf{1}}$ & $\mathbf{O I}^{\mathbf{m}}$ & $\mathbf{I R}^{\mathbf{n}}$ & $\mathbf{O R}^{\mathbf{0}}$ & & & & & \\
\hline Farmers & 4.67 & 4.42 & 1.89 & 0.20 & 1.88 & & & & & \\
P\&R & 7.87 & 5.91 & 3.98 & 1.12 & 1.99 & & & & & \\
\hline
\end{tabular}

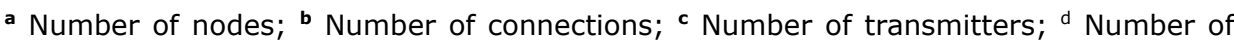
receiver; ${ }^{\mathbf{e}}$ Map density; ${ }^{\mathbf{f}}$ Mean in-degree of Driver; ${ }^{\mathbf{g}}$ mean out-degree of Driver; ${ }^{\mathbf{h}}$ mean In-degree of pressure; ${ }^{\mathbf{I}}$ mean Out-degree of pressure, ${ }^{\mathbf{j}}$ mean in-degree of State; ${ }^{\mathbf{k}}$ mean out-degree of State, ' mean in-degree of Impact, $\mathbf{m}$ mean out-degree of Impact, $\mathbf{n}$ mean in-degree of Response; ${ }^{\circ}$ mean out-degree of Response.

\subsubsection{Simulation and what-if analysis results}

Currently there are different policy alternatives being considered by policy makers in Rafsanjan. As an example, we simulate the impact of the four different policy options that have been explained in section 3.2.4. After running each policy option, the end values of each option were compared with the end 
value of the baseline steady state. Figure 3.6 indicates the positive and negative changes of various concepts for each policy option. Concepts without change are not shown in Figure 3.6.

Policy option 1: The economic change policy has the largest influence on decreasing water shortage in the farmers' model. Although this policy decreases agriculture production as well, eventually it results into sustainable economic development in the region. This interesting result indicates that farmers have a high acceptance in a change of regional economy from agriculture to other source of economies.

Policy option 2: The irrigation and agriculture system modification policy has a large influence on a pistachio production increase according to the farmers' model, but it does not help the water scarcity problem that much. It explains that the current agriculture/irrigation system is one of the main drivers of water shortage, but the policy for its modification only works as a short-term solution to palliate the symptoms of the problem, i.e. pistachio production, without removing the main cause. This policy does not have any significant influence on the P\&R model except increasing the drying up of orchards.

Policy option 3: The people participation and integrated land owning also has a large influence on pistachio production growth in the farmers' model, but it has the lowest positive impact on water shortage in both models. From the farmers' point of view the policy of people participation and integrated land owning can highly improve government control.

Policy option 4: Although the farmers perceive lack of government control as one of the main drivers of water shortage, they do not believe that control and monitoring policies decrease the water shortage of the region as much as economic change and irrigation modification policies. It does not have any influence on impact concepts as well. On the contrary, the government control and monitoring is the most effective policy option in P\&R perceptions. It has a much larger positive impact on water shortage than other policy options, as well as on unregulated ground water use and orchards dry-up. Additionally, this policy option dramatically increases sustainable investment of the region according to the perception of the P\&R group.

To conclude, the economic change and government control and monitoring policies are considered to be the most effective policy options fighting water scarcity. However, farmers do not perceive current government policies to control, monitor and constraint water use as very effective. 


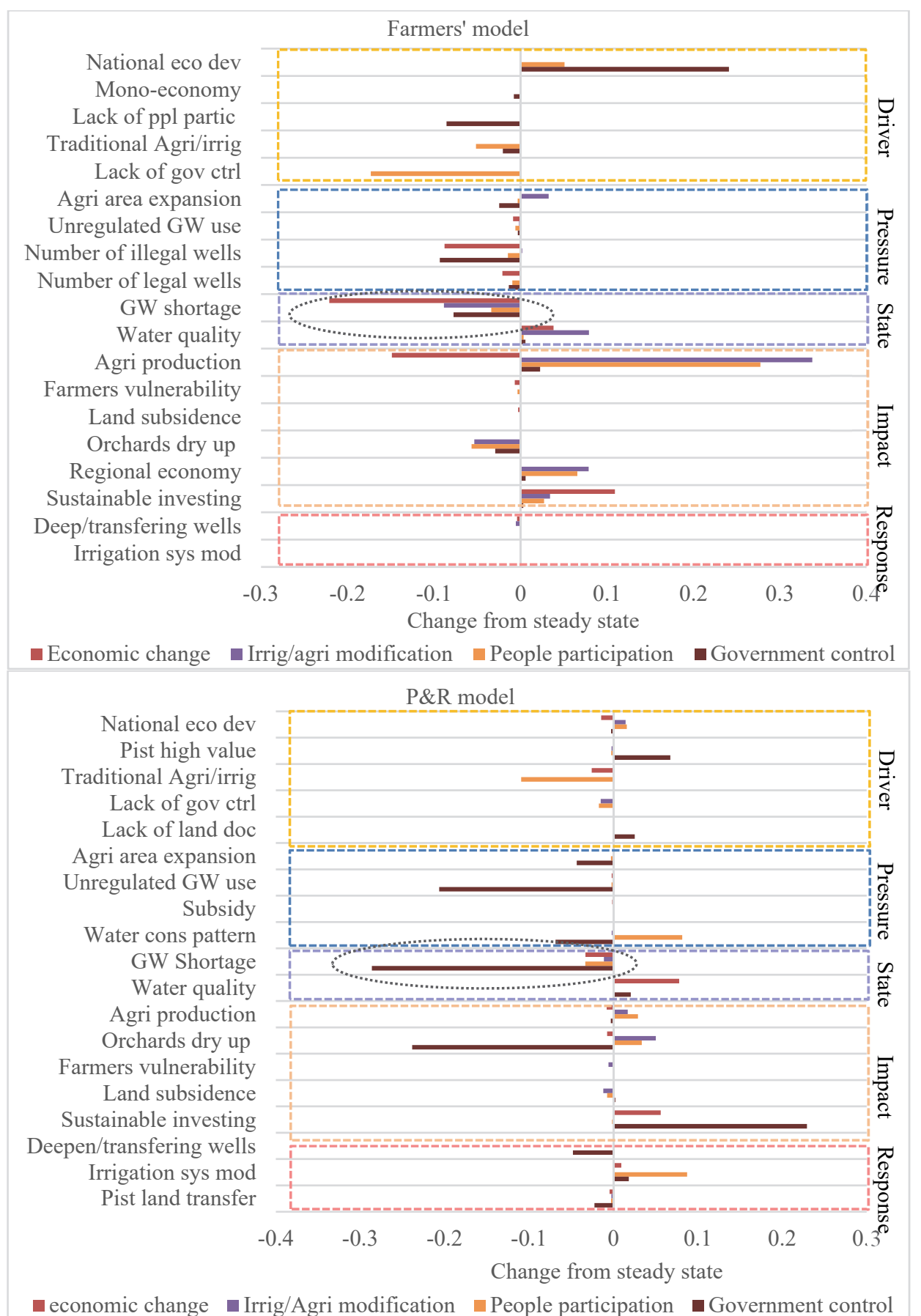

Figure 3.6: Simulation results of four policy options and their impact on each indicator compared to the baseline steady state for P\&R and farmers' models. The horizontal values represent the change in value of each variable for each policy scenario. Negative value. 


\subsection{Discussion}

Related work: Some studies use causal networks rather than causal chains in an Enhanced-DPSIR to represent inter-relations of complex systems and to indicate the importance or priority level of concepts based on stakeholder opinions, statistical data and literature (Niemeijer and de Groot, 2008, Namaalwa et al., 2013). Yet, as the degree of causal links is not described in this model, it cannot capture trends but only provide a snapshot picture of a constantly changing system. Other studies use a Bayesian Belief Network (BBN), a probabilistic graphical modelling technique, in DPSIR to quantify causal relationships (Langmead et al., 2009, Lowe et al., 2014). While BBN is a directed acyclic graph it does not incorporate feedback cycles, which are a crucial part of SES (Carvalho, 2013). In our methodology, we proposed to use FCM in combination with the DPSIR framework to overcome these limitations. Mourhir et al. (2016) also introduced an environmental assessment mixmethodology using DPSIR and FCM. Our approach differs from theirs in several ways. Mourhir et al. (2016) used DPSIR to predefine and frame concepts via a stakeholders' workshop and data analysis, and then used the standardized concepts for FCM generation via individual experts. For a SES setting like Rafsanjan, we found it difficult and confusing to structure the problem through defining the variables of DPSIR with stakeholders. Rather, we used a free-style interview for FCM to capture as diverse a range of concepts as possible and then used DPSIR to reduce and structure the complexity of the resulting FCMs. Further, unlike Mourhir et al. (2016), we used the DPSIR framework in later steps of our methodology, for condensation, and as a means to structure the analysis and simulation.

Applicability of method: The aim of this research was to incorporate stakeholder perception in policy option analysis in the context of a SES problem. The proposed mix-method was easy to use for the acquisition of knowledge from a large group of stakeholders and understanding and analysing the impact of different policy alternatives. For future studies, our structured participatory method is applicable in cases where there is 1) a heterogeneous group of stakeholders with strong conflicting interests, and 2) divergent knowledge of problem indicators among stakeholders and scientific studies. In this respect, mere reliance of policy makers on available scientific objective data may result into non-effective policy options for stakeholders. Therefore, our structured participatory method is applicable in SES problem settings having the two earlier mentioned characteristics. Application of our method requires the following conditions: 1) ability to identify and categorize groups of stakeholders with different interests, 2) willingness of stakeholders to provide their perceptions in a comprehensive survey, and 3) availability of literature data to categorize each of the DPSIR indicators. If that is the case, the proposed mix-method allows for simulation of different policy options. 
The inherent limitations and constraints of each method define the context and extent of its application. An important applicability feature of our method is related to the kind of participatory data collection technique that is used in FCM and makes it different from the knowledge engineering methods (Jetter and Kok, 2014). Unlike the latter, which focuses on capturing the knowledge of recognized experts and presenting it to other people (Jetter and Kok, 2014), the former tries to extract the knowledge of as many stakeholders as possible in an equivalent manner (Papageorgiou and Kontogianni, 2012). The outlier concepts, though these might be important variables but just not perceived by many respondents, are eliminated in condensation or given little weight in social maps. Although there are methods for scaling respondents' contributions or assigning credibility weights to experts (Kosko, 1987), in our view, participatory FCM does not aim to represent reality itself but the most dominant perception of reality. In this way, it is a good tool to simulate the general acceptance and suitability of policy options based on stakeholders' perceptions and experiences. It might happen that policy concepts with strong engineering and technical support of success do not receive positive perception from some of the important stakeholders. In such cases, our method will identify stakeholders' perceptions and recognize discrepancies in perceived policy impacts that might be valuable in reconsidering policy options in order to achieve more support from stakeholders.

Non-applicability: Nevertheless, there are three main shortcomings in the participatory FCM method that need future study:

1) In the case study we noted that some part of the system cannot be observed/perceived by people but there might be accurate scientific data about them, e.g. land subsidence or ground water level which are known as hidden problems. This deficiency of participatory FCM may require a mixed-method that combines local-knowledge-based and scientific-knowledge-based FCMs.

2) The method relies on realities that have been experienced and perceived by respondents. Therefore, only impacts of policy options that have already been experienced or have been well explained can be assessed. The impact of a "water transfer project" is an example of a policy option that cannot be assessed with this method, if the consequence is not well presented and made known, because there is no experience yet with importing (highly priced) water to the region. For using FCM as a policy making tool more modifications and enhancements are required.

3) Since the perception of the different stakeholders is taken, mapped and aggregated by the mediator/interviewer, his/her skill on this also plays a very important role to the final results. It may cause the biased and subjective results especially in the cases that interviewer is not involved enough in the stakeholders' communities. 


\subsection{Conclusions}

This study introduces a method to model a complex SES problem and to assess impacts of different policy options. It has been applied on a SES setting related to water scarcity in Rafsanjan, Iran. Combining the problem structuring method of DPSIR with a participatory modelling method of FCM gives us the opportunity 1) to structure the system problem and avoid over-complexity of the participants cognitive maps, 2) to elicit data in cases with lack of reliable data, 3) to involve different stakeholders with conflicting interests and develop perception models, and 4) to simulate impacts of policy interventions on different components of the SES.

The case of Rafsanjan clearly needs two types of strategies to increase its resilience in dealing with water scarcity. First, the quick and short-term actions to fix the most urgent problems, like losing pistachio lands, and to keep the region a place for farmers to stay and invest. Second, the longer-term solutions to secure the sustainable groundwater management in Rafsanjan. The results of a policy option impact analysis indicate that change of economy and government control and monitoring are perceived as being the most effective water scarcity policy options by farmers and P\&R respectively. However, the modelling clearly showed that the farmers have a low level of trust in the ability of the government to regulate and control water usage. The current water crisis appears to justify such a view. A crucial issue for effective water management in Rafsanjan would therefore be an equitable engagement of all major stakeholders so that a multi-stakeholder approach to sustainable groundwater management can be pursued.

Finally, the outcome model of this study can only be used for deliberating and comparing the acceptability of different policy options' impacts, and is not meant to give definitive answers to the problem nor accurate forecasts of policy impact. 


\section{Chapter 4*}

\section{Integrating Perception-Based and Data-Driven Knowledge to Support Policy-Making in Social-Ecological Systems: A Fuzzy Cognitive Mapping Approach}

* This Chapter is based on:

Mehryar, S., Sliuzas, R.V., Reckien, D., Sharifi, A. and van Maarseveen, M.F.A.M., 2018. Integrating Perception-Based and Data-Driven Knowledge to Support PolicyMaking in Social-Ecological Systems: A Fuzzy Cognitive Mapping Approach. Regional environmental change (manuscript under review). 


\begin{abstract}
In modelling Social-Ecological Systems (SESs) social and ecological variables interact, implying that both subjective and objective data are crucial, complementary and need to be integrated to enable a full understanding of the system for policy making purposes. Fuzzy Cognitive Mapping (FCM) is a wellknown participatory modelling method that uses stakeholders' perceptions to build semi-quantified models. We develop an FCM model for policy option analysis in a SES by combining two types of knowledge from formal objective data and stakeholders' perceptions. This model is focussed on the issue of water scarcity in Rafsanjan, Iran. It includes many social and ecological variables, and allows the impact of different policy options on the system to be simulated. The simulation results of the mixed-FCM have been compared with those of a standard, perceived FCM (P-FCM), of the same SES. The results show that when simulating policies with direct impact on data-driven concepts, the mixed-FCM produces substantially different results from those of a P-FCM, thereby showing the benefit of this approach in such settings. Yet, for policies with direct impact on perceived concepts both mixed-FCM and P-FCM produce similar simulation results, which support continued use of P-FCM for these type of policies. Therefore, a mixed-FCM is useful for the study of SESs in which part of environmental changes are gradual and invisible to stakeholders' direct observation e.g. groundwater level change. Whereas, for the study of environmental changes that can be perceived immediately by stakeholders over relatively short time frames, a P-FCM would suffice.
\end{abstract}

Keywords: Participatory modelling, Social-ecological systems, Policy making, Fuzzy Cognitive Mapping, Water Scarcity 


\subsection{Introduction}

Environmental management and policy-making require new methods that allow an integrated understanding of Social-Ecological Systems (SESs) (Walker et al., 2004). In such systems, traditional environmental management methods which rely on formal objective data often fail to consider the behaviour and interaction of all variables, especially the social variables for which formal objective data may be lacking (Ascough et al., 2008, Papageorgiou et al., 2016). In response, participatory approaches in management of SESs have emerged (Virapongse et al., 2016), such as participatory modelling that uses stakeholders' knowledge and perceptions in SES scenario modelling (Voinov and Bousquet, 2010, Basco-Carrera et al., 2017). Examples of such participatory modelling approaches are: Bayesian belief networks (Castelletti and Soncini-Sessa, 2007, Henriksen et al., 2007), Fuzzy Cognitive Mapping (Özesmi and Özesmi, 2003, Papageorgiou and Kontogianni, 2012), and Agent-Based Modelling (Campo et al., 2010, Ansell and Gash, 2008). According to these studies, integration of diverse local knowledge in modelling is helpful to 1 ) represent multiple interests of various stakeholders; 2) identify the likely impact of policy solutions by stakeholders; 3) foster individual and group learning to improve communication, trust building, and collective action (Voinov and Bousquet, 2010, Gray et al., 2012). In spite of these advantages, sole reliance on stakeholders' knowledge in SES modelling also has shortcomings. For example: 1) participatory modelling may be highly subjective and lack empirical evidence to support the claims; 2) the public may be ill-equipped to understand and represent the complexity of SESs; and 3) people's perceptions are based on their particular experience at a particular space and time (Gray et al., 2012, Elsawah et al., 2015). These pros and cons indicate that in modelling a SES we have to deal with two types of variables;

Perception-based variables: variables that can be better perceived by stakeholders, due either to a lack of formal scientific data or to the relative importance of stakeholders' observation and local knowledge for specific variables given the research problem;

Data-driven variables: Variables that can be better measured by formal scientific data, due to their availability and the requirement for standardised, accurate measurements e.g. precipitation and groundwater level.

This means that the subjective knowledge derived from stakeholders and the objective knowledge derived from formal scientific studies are complementary in modelling of SESs. 


\subsubsection{Background}

In this study, we investigate the SES of pistachio production in Rafsanjan, Iran. It suffers from serious water scarcity, due to a multitude of poorly understood, but strongly interacting social and ecological variables as drivers and impacts (Mehryar et al., 2016). Policy-making, in this case, should consider socioeconomic variables such as pistachio production, farmers' vulnerabilities, immigration, and regional economy as well as technical and ecological variables such as climate change, groundwater exploitation, land subsidence, and land cover change. It should, therefore, deal with a complex set of changing variables, variable knowledge about all aspects of the system and uncertain consequences of conflicting interventions e.g. to maintain the production of region, or to protect the aquifer, or to maintain public satisfaction levels.

(Mehryar et al., 2017) built a participatory FCM, using two groups of stakeholders, i.e. policy makers and farmers, to elicit unavailable or intangible data from local stakeholders and enable the comparison of farmers' and policy makers' perception models. They interviewed 60 farmers, and 40 policy makers (from water, agriculture, and environment authorities as well as the research institutes responsible for providing scientific reports and studies for policy makers) about: 1) the current state of the water situation in Rafsanjan; 2 ) the social-ecological causes and impacts of this water situation; and 3) their adaptive responses to the situation. Results were mapped individually during the interviews by a local facilitator and later combined into two FCMs, one for each group of stakeholders. These two FCM's were developed and analysed separately for simulating the impact of different policy options (Mehryar et al., 2017).

Despite the usefulness of the FCM method in addressing data scarcity by integrating stakeholders' knowledge, some of the available scientific knowledge was missing in the final model, due to the methodological limitation of FCM in integrative use of subjective and objective knowledge. There were some parts of the SES for which scientific data could more accurately reveal changes of variables than is possible through stakeholders' observations. For example, land subsidence and groundwater level change are relatively slow changing phenomena that may not be easily detected in short-term and without sophisticated instruments. Therefore, the influence of these changes on the system may not be perceived by people whereas scientific data can detect or predict them. In line with the authors' suggestion for future studies, decision making in such a SES may benefit from a mixed approach that combines local knowledge with formal knowledge in one model to simulate the possible impact of future policy options. 


\subsubsection{Objective}

Our objective is to develop and demonstrate an approach of using both formal knowledge i.e. knowledge derived from scientific objective data and perception-based knowledge i.e. local knowledge from stakeholders in a single SES model. We combine these two knowledge forms in one FCM model and then compare the results with those of a standard FCM method that use only perception-based knowledge.

\subsubsection{Technical background: Fuzzy Cognitive Map}

The FCM method, introduced by (Kosko, 1986a) is a powerful tool to investigate the behaviour of SESs by simulating the dynamics of causal relations among complex variables. An FCM is a signed directed graph with feedback, consisting of nodes and directed edges with weights (Carvalho, 2013). Nodes (concepts) represent the variables of the system, which can be social, economic, ecological, or abstract concepts. Directed edges (connections) connect nodes and represent causal relations between concepts. Each connection has a direction and is associated with a weight ranging from - 1 to 1 ; a positive weight represents a causal increase and a negative weight represents a causal decrease between concepts (Carvalho, 2013). FCM's graph structure facilitates building simulations using forward inference algorithms. By FCM simulation with an initial state, it models the evolution of system over time, letting concepts interact with each other, and produce projections by evolving forward (Özesmi and Özesmi, 2004). FCM simulations can be used for "what-if" analysis in different possible scenarios of a given system (Mourhir et al., 2017).

The FCM method was primarily developed for perception-based approaches where a researcher elicits mental models of stakeholders via individual or group interviews or -less often- via questionnaires (Gray et al., 2014, Matmir et al., 2017). FCM has been proven an especially useful approach in cases for which data is scarce or fragmented, and stakeholders have a profound knowledge of the system behaviour e.g. in SES and environmental modelling (Olazabal et al., 2018). Due to the high dependency of this approach on stakeholders' beliefs and judgments that can be rather subjective or include potential bias of the human experts (Papageorgiou and Salmeron, 2013), in recent years new FCM algorithms have been developed to support learning of FCMs from time-series data. In such algorithms the model mimics historical time series and defines FCM structure i.e. main concepts, causal relationships (edges), and their weights solely based on historical data and without any or with relatively limited human intervention (Stach et al., 2005, Papageorgiou and Salmeron, 2013, Papageorgiou, 2012). Yet, such data-driven FCMs have been mainly used in technical, industrial, or medical domains like water demand forecasting (Papageorgiou et al., 2015, Salmeron et al., 2016), 
industrial process control (Baykasoglu et al., 2011), and genetic (Liu et al., 2017), where large time-series data are available for modelling.

In SES and environmental studies, where empirical data are less available but still crucial for modelling the system, few studies have made use of formal knowledge as input to an FCM. Kok (2009) used input-data from literature to introduce an FCM for a simulation of Brazilian Amazon deforestation. He applied a mix between classifying and ranking methods to assign the values of relationships from input-data. Soler et al. (2012) used spatial data sets to build an FCM for developing land cover scenarios of the Brazilian Amazon. They used the correlation matrix to estimate the strength of relationships and then incorporated expert-FCM to validate their results. Van Vliet et al. (2017) used mathematical models to create a formal FCM that was compared with a stakeholder-based FCM of the same system. In this study we follow the Soler et al. (2012) method in assigning weights to the connections between datadriven concepts, although our general approach in building a mixed-FCM differs from theirs. The novelty of our approach lies in how to integrate and represent both stakeholders' knowledge and data-driven knowledge in a single SES model. This is particularly useful in cases where both qualitative and quantitative data are important and complementary in representing a SES's complexity.

\subsection{The Methodological Framework}

The five-step methodology of this study (see Fig1) extends our earlier study in (Mehryar et al., 2017). This methodology demonstrates how to simulate the possible impacts of policy options by combining data-driven FCM (D-FCM) and Perceived FCM (P-FCM).The sequence of methodology steps are shown in fig1. The meaning of the acronyms used in the methodological framework as well as the remaining of the paper are as follows:

P-FCM: Perceived FCMFP-FCM: Farmers' Perceived FCM

PP-FCM: Policy makers' Perceived FCM

CP-FCM: Combined Perceived FCM (farmers and policy makers)

D-FCM: Data-driven FCM

M-FCM: Mixed-FCM (Perceived-Driven FCM) 


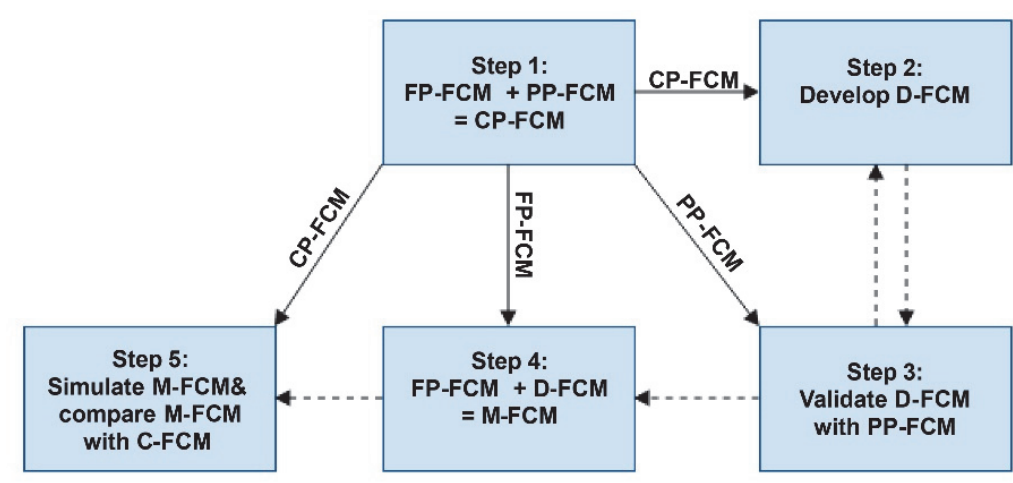

Figure 4.1: Conceptual framework of methodology.

Each step is described in more detail below.

\subsubsection{Step 1: Define FCM structure via perceived knowledge}

The purpose of this step is to define the main structure of FCM i.e. important concepts, their causal connections and weights with participatory-based approach and develop a P-FCM. Semi-structured interviews with open-ended and free-style questions are the most useful technique for this methodology. In this technique, the interviewees choose their own concepts without any predefined structure, and the questions cover the whole aspects of the problem including the parts that can be modelled via empirical data e.g. meteorological and hydrological changes. For instance, guiding questions can be: what are the causes and impacts of the social-ecological problem (natural resource problems, climate change, etc) in your region, and how much have been the impacts of these variables on each other. In this step, the most prominent causal relationships between concepts can be collected and triangulated via crowdsourcing. Such relationships might be difficult to recognize only via empirical data (Step 2).

Two points should be noted in developing a P-FCM for this methodology:

First, P-FCMs should be developed from two different sources of knowledge 1) knowledge obtained by local experience in the system e.g. knowledge of inhabitants, farmers or local natural consumers, and 2) knowledge obtained by secondary literature, government reports, and scientific studies e.g. knowledge of researchers, scientists, and policy makers. Former is useful in covering the data-poor part of the model i.e. perception-based variables, and the latter can fill the more data-abundant parts of the model, including evaluating results of the D-FCM developed in step 3. 
Second, in order to combine P-FCMs with D-FCM it is needed to specify the exact time scale for each concept and relationship. For instance when the interviewer asks about the relationships between precipitation and agriculture area expansion, it should be explicit whether the question is for short-time changes e.g. 10 years, or long-time changes e.g. 50 years.

In this study we combined the P-FCM model of farmers i.e. FP-FCM with that of policy makers i.e. PP-FCM (Mehryar et al., 2017) to create one CP-FCM that represents the entire SES (Figure 2). For this combination, all farmers' and policy makers' individual matrices were combined through matrix algebra, whereby each entry of the combined model is the average of all the weights for that entry provided by all interviewees. Then we identified the exact temporal scale of each variable based on interview questions. For instance impacts of climate change, population growth and pistachio land expansion on other variables were asked for the last thirty or forty years, since their impacts are revealed over a long period. Whereas, impacts of groundwater level and groundwater quality were asked only for the last ten years since they have shown significant changes over shorter periods.

\subsubsection{Step 2: Develop a D-FCM}

The purpose of this step is to extract the data-driven concepts and connections from CP-FCM, calculate their weights with objective data, and build D-FCM.

\section{Define data-driven concepts}

First, all the concepts with available time-series data should be identified and extracted from CP-FCM. To be consistent with the CP-FCM, similar temporal scales are applied in collecting time-series data for same concepts i.e. the last ten years for short-term variables and thirty to forty years for long-term variables depending on the nature of each variable. In this step, a researcher should have a proper understanding of the systems' variables to identify which variables can be better perceived by stakeholders or measured by objective data. However, definition of nature and behaviour of variables is a case specific and problem specific issue. For this case study, we provided a typology of all CP-FCM variables i.e. specifying perceptible and data-driven variables before building the D-FCM (appendix 6 ).

The data-driven variables with their available time-series data and resources are shown in figure 2 and table 2. 


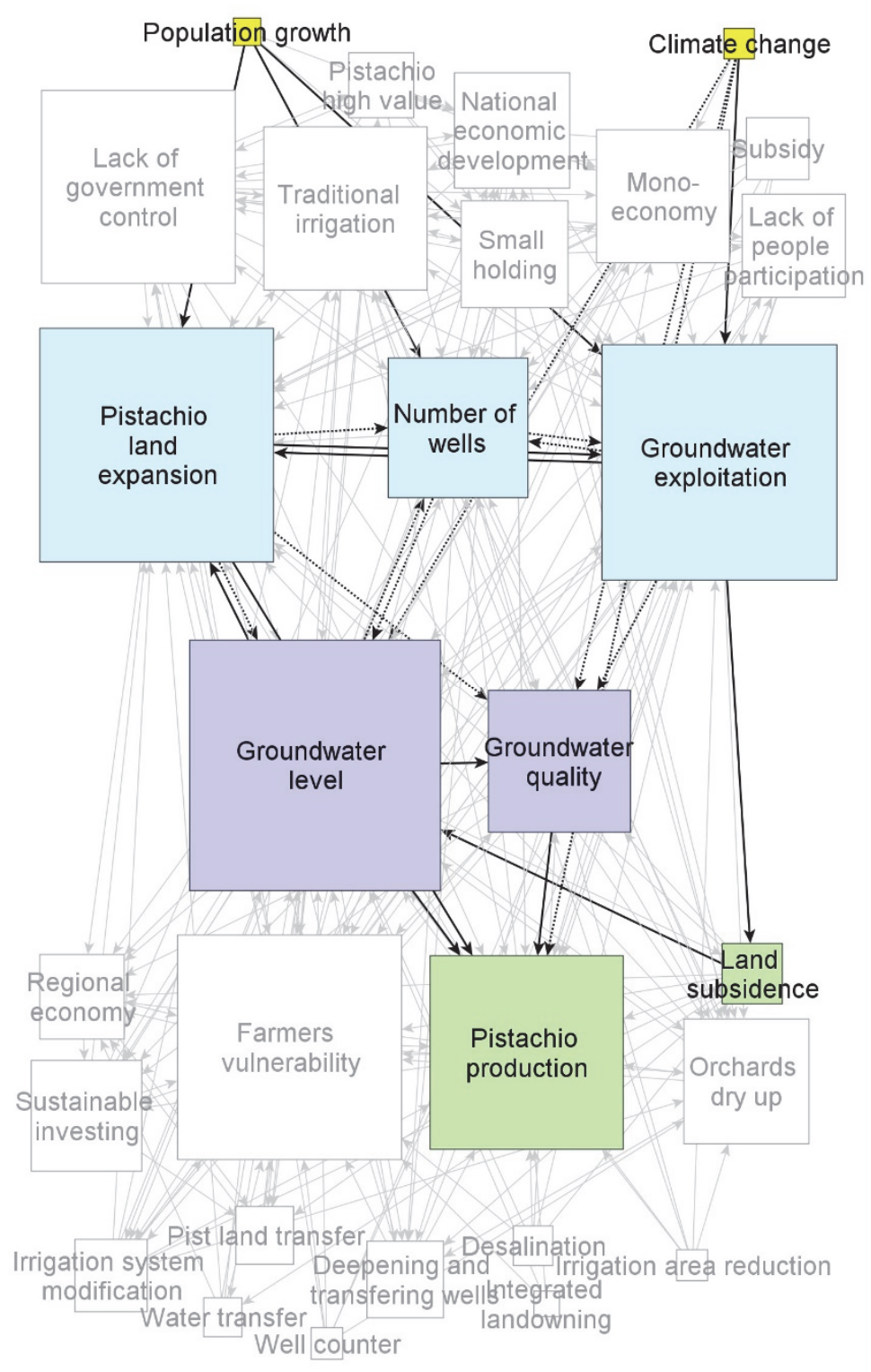

Figure 4.2: The CP-FCM of all stakeholders: Coloured concepts and black edges represent data-driven variables and data-driven connections respectively 
Table 4.1: Description of variables with available time-series data

\begin{tabular}{|c|c|c|c|}
\hline $\begin{array}{l}\text { Variable } \\
\text { name }\end{array}$ & Description & Years of data & Source \\
\hline $\begin{array}{l}\text { Climate } \\
\text { change } \\
\text { (precipitation } \\
\text { and heat) }\end{array}$ & $\begin{array}{l}\text { Annual precipitation } \\
\text { (mm) and average } \\
\text { monthly maximum } \\
\text { temperature }\end{array}$ & $\begin{array}{l}1982 \text { to } 2016 \\
\text { annually }\end{array}$ & $\begin{array}{l}\text { Iran Meteorological } \\
\text { Organization (IRIMO) }\end{array}$ \\
\hline $\begin{array}{l}\text { Population } \\
\text { growth }\end{array}$ & & $\begin{array}{l}\text { 1966- 1986- 1996- } \\
\text { 2006- 2011- } 2016\end{array}$ & $\begin{array}{l}\text { Iran National Census } \\
\text { https://www.amar.org } \\
\text {.ir/english/Iran-at-a- } \\
\text { glance/Kerman }\end{array}$ \\
\hline $\begin{array}{l}\text { Pistachio land } \\
\text { expansion }\end{array}$ & $\begin{array}{l}\text { Area of total pistachio } \\
\text { lands (ha) }\end{array}$ & $\begin{array}{l}\text { 1976- 1986- 1998- } \\
2006-2014\end{array}$ & $\begin{array}{l}\text { Landsat } 8 \text { images - } \\
\text { NDVI (Mehryar et al., } \\
\text { 2015) }\end{array}$ \\
\hline $\begin{array}{l}\text { Number of } \\
\text { legal wells }\end{array}$ & & $\begin{array}{l}\text { 1971- 1973- 1974- } \\
\text { 1975- 1976- 1981- } \\
\text { 1983- 1986- 1989- } \\
1993-1997-2005- \\
2006\end{array}$ & $\begin{array}{l}\text { Official reports, Water } \\
\text { research institute, } \\
\text { Ministry of Energy }\end{array}$ \\
\hline $\begin{array}{l}\text { Groundwater } \\
\text { exploitation }\end{array}$ & $\begin{array}{l}\text { Sum of the annual } \\
\text { groundwater } \\
\text { exploitation by total } \\
\text { wells ( } \mathrm{mm} 3 \text { ) }\end{array}$ & $\begin{array}{l}\text { 1971- 1973- } 1974- \\
\text { 1975- 1976- } 1981- \\
1983-1986-1989- \\
1993-1997-2005- \\
2006\end{array}$ & $\begin{array}{l}\text { Official reports, Water } \\
\text { research institute, } \\
\text { Ministry of Energy }\end{array}$ \\
\hline $\begin{array}{l}\text { Groundwater } \\
\text { level }\end{array}$ & $\begin{array}{l}\text { Average of the annual } \\
\text { groundwater level of } \\
\text { the whole Rafsanjan }\end{array}$ & $\begin{array}{l}1983 \text { to } 2013 \\
\text { annually }\end{array}$ & $\begin{array}{l}\text { Official reports, Iran } \\
\text { water resources } \\
\text { Management Company } \\
\text { http://wrbs.wrm.ir/ }\end{array}$ \\
\hline $\begin{array}{l}\text { Groundwater } \\
\text { quality }\end{array}$ & $\begin{array}{l}\text { Average of the annual } \\
\text { Electrical Conductivity } \\
\text { (EC) of groundwater in } \\
\text { Rafsanjan }\end{array}$ & $\begin{array}{l}1998 \text { to } 2015 \\
\text { annually }\end{array}$ & $\begin{array}{l}\text { Official reports, Iran } \\
\text { water resources } \\
\text { Management Company } \\
\text { http://wrbs.wrm.ir/ }\end{array}$ \\
\hline $\begin{array}{l}\text { Pistachio } \\
\text { production }\end{array}$ & $\begin{array}{l}\text { Sum of production in } \\
\text { the whole Rafsanjan }\end{array}$ & $\begin{array}{l}1982 \text { to } 2015 \\
\text { annually }\end{array}$ & $\begin{array}{l}\text { Iran Pistachio } \\
\text { Association } \\
\text { iranpistachio.org/fa/sa } \\
\text { mple/before }\end{array}$ \\
\hline $\begin{array}{l}\text { Land } \\
\text { subsidence }\end{array}$ & & $\begin{array}{l}2004 \text { to } 2016 \\
\text { annually }\end{array}$ & $\begin{array}{l}\text { Scientific studies } \\
\text { (Motagh et al., 2017) }\end{array}$ \\
\hline
\end{tabular}

\section{Define data-driven connections}

Among all possible connections between data-driven concepts those should be selected that fulfil either of two conditions: 1) being already in the CP-FCM, which means existence of a causal relation is already verified through crowdsourcing, or 2) being mentioned in formal studies (government and organizations' reports, research centres' studies, scientific publications) which means their causal relationship has been noticed in (scientific) literature.

In our study, most of the connections corresponded to the former condition. The causal relation between land subsidence and groundwater level was the only relation that fits the second condition, i.e. it was observed in studies but was not taken into account by stakeholders. This relationship will be discussed more in section 3.1. 


\section{Add weights to data-driven connections}

To estimate the strength of relationships between concepts a correlation coefficient matrix was used following the approach of Soler et al. (2012). For each selected connection, the Pearson Correlation between the time series of two variables has been calculated. Then only the connections with a significant Pearson correlation ( $p>0.05$ at two-tailed test) have been selected and all others have been excluded from the map.

To be consistent with the former CP-FCM as reported in Mehryar et al. (2017) we ranked the correlation values in a scale between 0.1 and 0.9 to represent the connections' weights. Thus, the highest correlation value received value of 0.9 assuming that a perfect link between two variables (value of 1.0 ) does not exist in SESs due to the complex cause-effect relationship of the whole system (Kok, 2009, Soler et al., 2012). The lowest correlation values received value of 0.1 indicating that relationships $<0.1$ are assumed to be almost non-existent if stakeholders and studies have noticed a relationship.

We used linear interpolation for missing data in those time series having regular (linear long-term) trends of change e.g. population growth and pistachio land expansion that have continuous increase or decrease. Yet, for the concepts with irregular trends of change (high frequency fluctuations) e.g. groundwater exploitation and pistachio production, we have only used available time-series data. A list of calculated measured-weights is shown in table 4 (section 3.1).

\section{Build the D-FCM}

The final step in building the D-FCM is to bring together all selected concepts and their weighted causality into an adjacency matrix. We used FCMapper (http://www.fcmappers.net/joomla/) to run the model and calculate a steady state situation. All initial values of D-FCM concepts were set to 1 , assuming they are all equally active or important. Then the evolution of an FCM over time is modelled iteratively. In each iteration (time step), the value of each concept is multiplied by the respective weight through matrix multiplication; then the results are passed through a non-linear, so called "squashing" function to limit the range of possible output values yielded as weight for the next iteration (Reckien et al., 2013, Mehryar et al., 2017). This procedure in FCM models is repeated in iterations until the resulting value reaches equilibrium i.e. converges to a fixed-point (Özesmi and Özesmi, 2004), but theoretically they can also settle into a finite cycle of states, or chaotic attractor (Dickerson and Kosko, 1994). 


\subsubsection{Step 3: Validation via triangulation}

Although the causal connections of the D-FCM have been developed and verified by participatory approach, still an inherent uncertainty in the link between weights and Pearson correlation might be present e.g. due to inaccuracy of data, or correlations that might not be because of causation. To evaluate such uncertainties, we used the PP-FCMs for validation of the D-FCM via triangulation. In a simple sense, triangulation is the use of multiple methods and observers -quantitative and/or qualitative- to study a specific problem. It is a powerful technique to validate results of one and the same subject through cross verification from two or more data sources and/or methods (O'Donoghue and Punch, 2003).

Policy makers' knowledge - in the case of Rafsanjan - is predominantly based on formal reports and scientific studies, including scientific observations or measurements (Mehryar et al., 2017). Therefore, their FCM i.e. PP-FCM is compared with the D-FCM to define similarities and significant differences between the two sources. The result of this comparison is discussed in section 3.1.

\subsubsection{Step 4: Combine the D-FCM and P-FCM}

To build a M-FCM, we combined the FP-FCM - as perceived part of the model - with the D-FCM. The FP-FCM was chosen because most concepts identified as perception-based concepts (appendix 6) are inherently experienced and observed in practice by the farmers who are the local experts on the system e.g. impact of subsidy, small holding, farmers' vulnerability, irrigation system modifications etc.

The FP-FCM was used as base, then the connections validated as data-driven connections (step 3) received data-driven values, yet all other connections (with either one or both side perception-based concepts) remained with their perception-based values. The new data-driven connections were simply added to the matrix. Resulting M-FCM is represented in section 3.1.

\subsubsection{Step 5: Simulate with the M-FCM model}

With the M-FCM model it is possible to simulate different policy options for which both data-driven and perception-based variables can be modified. MFCM simulation is done by feeding the model with stimulus inputs, analysing the evolution of a system over time through the interactions of all concepts. For each policy option, the relevant concepts are modified, or sometimes concepts are added or removed. This approach allows asking "what-if" questions for each change in concepts that represent new policy option interventions. 
In this paper we simulated the impacts of four policy options that have been used in the former study (Mehryar et al., 2017) and are shown in table 3. These options reflect the available solutions that were under discussion by the government to solve the water scarcity in Rafsanjan. In each policy option run two or three concepts related to that policy option were clamped to a value between 0 and 1 . If a policy option increases the influence of a concept, it is clamped to 1 and if it permanently decreases the influence of a concept, it is clamped to 0 . The result of this simulation is presented in section 3.2.

Table 4.2: Policy options, the clamped concepts and their values in each policy option.

$\begin{array}{lll}\text { Policy option } & \text { What-if question } & \begin{array}{l}\text { Clamped concepts and } \\ \text { their clamped values }\end{array}\end{array}$

1) Economic change What if the economy of the Pistachio land expansion region changes from (data-driven concept) $=0$ agriculture to other branches Pistachio production (datalike industry and service driven concept) $=0.5$ production? Mono-economy (perceived Mono-economy (perceived
concept) $=0$

2) What if the decision making Irrigation/Agriculture focuses on modification of system change the irrigation/agriculture Agriculture area expansion (data-driven concept) $=1$ the irrigation/agriculture Traditional system by using advanced Agriculture/irrigation technology to improve (perceived concept) $=0$ production? Pistachio production (datadriven concept) $=1$

3) People participation and integrated landownership

What if the decision-making

Lack of people participation focuses more on participatory management methods? Integrated (perceived concept) $=0$ landowning for optimized irrigation and agriculture is one of the main solutions that needs farmers' high Small holding (perceived concept) $=0$

Community based organization (perceived concept) $=1$ involvement.

4) Government
control, monitoring
and limitation

What if the government implements appropriate controlling and monitoring policies?

Subsidy (perceived concept)
$=0$
Lack of government ctrl
(perceived concept) $=0$

\section{Compare the M-FCM and the CP-FCM}

Having the M-FCM and CP-FCM we can compare the result of their simulations as well as analyse the benefit of M-FCM method with respect to the standard P-FCM. Comparing these simulation results is presented in section 3.3. 


\subsection{Results}

\subsubsection{D-FCM structure, validation, and M-FCM structure}

By running D-FCM model in step 2.2.4, all concepts of the D-FCM reached a steady state within 40 iterations. The resulting D-FCM is visualised in a graphical form as illustrated in figure 3.

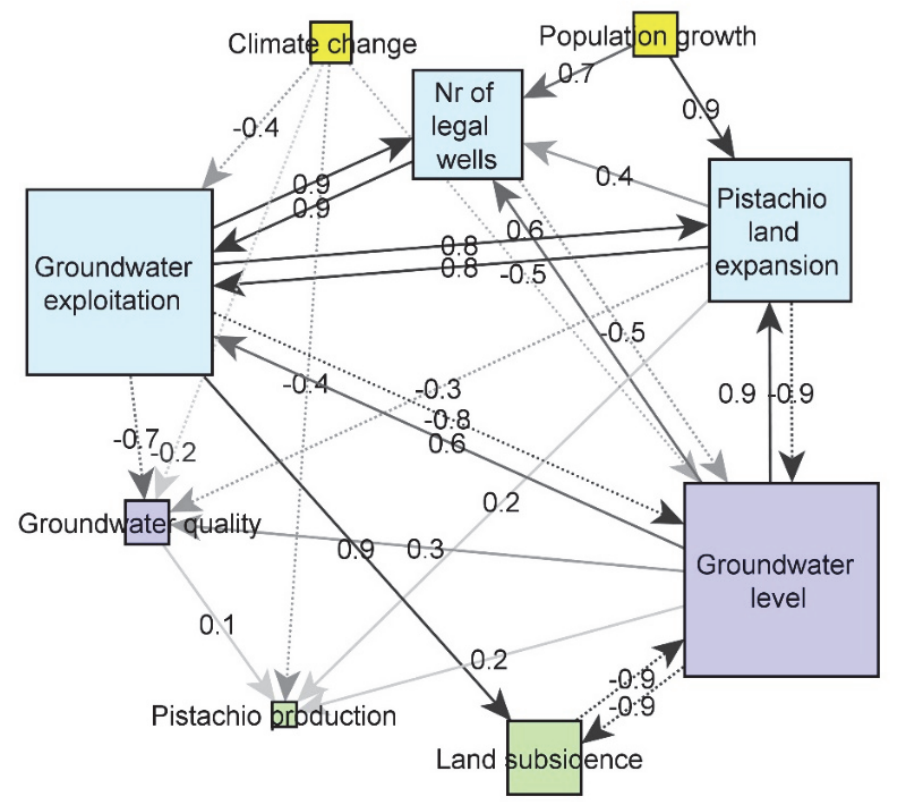

Figure 4.3: Graphical representation of the D-FCM. The size of the nodes indicates centrality (importance). Dotted and solid lines represent the negative and positive connection values respectively. Darker lines represent a higher connection value.

For comparing and validating the D-FCM result with PP-FCM, we focused on comparing concepts and connections that are shared among maps (figure 4). By comparing their connection values, six relationships showed significant value differences. Here value differences of $\geq 0.3$ are considered as significant. Considering the experts' discussions and literature studies these differences can be discussed as follows:

Groundwater overexploitation $\rightarrow$ Land subsidence \& Land subsidence $\rightarrow$ Groundwater level: Both of these relations have been underestimated by policy makers, whereas measured data reveal a strong positive and negative relationship for them respectively (connections $N \& Q$ in table 4 ). As explained earlier, overexploitation of groundwater reveals itself via land subsidence, which tends to occur slowly over long periods. It is relatively hidden from citizens' observations and thus often ignored, whereas fast-changing, easily observable phenomena (e.g. pistachio production) are perceived and 
considered to be more important (Chapin III et al., 2006). According to studies, groundwater extraction leads to land subsidence, which in turn leads to land deformation and the reduction of the groundwater reservoir volume. Consequently, the groundwater reservoir is no longer able to recharge water leading to a decline of groundwater level. In studies of Motagh et al. (2008) and Motagh et al. (2017) the strong relationships between land subsidence, groundwater level and groundwater use have been detected via InSAR timeseries data analysis. Therefore, for these two connections we relied on objective data and used the data-driven values for both of these connections in building DP-FCM.

Groundwater level $\rightarrow$ Groundwater quality: Policy makers believe a higher impact of groundwater level on Ground water quality than what their timeseries correlations show (connection S in table 4). The salinity of Rafsanjan's groundwater has always been high. In areas with lowest quality of groundwater i.e. eastern, southern and northern parts of Rafsanjan Township, pistachio yields are considerably effected by water salinity (Salehi and Hosseinifard, 2012). Yet the overall change of water salinity in Rafsanjan Township is not correlated with the change of ground water levels, contrary to policy makers' perceptions. The difference is probably due to the spatially specific nature of groundwater quality changes in Rafsanjan that effect some parts of Rafsanjan much more than on average. Since the aim of this study is to compare the impact of policies on the whole region rather than specific regions, we used the D-FCM values for this relationship to represent the general impact of groundwater level on groundwater quality for the whole Rafsanjan.

Pistachio land expansion $\rightarrow$ Pistachio production, Groundwater level $\rightarrow$ Pistachio production \& Groundwater quality $\rightarrow$ Pistachio production: Policy makers mentioned a relatively high influence of pistachio land expansion, groundwater level and groundwater quality on pistachio production, whereas in D-FCM Pistachio production does not show a significant change caused by changes of these three variables (connections $U, X, Z$ in table 4). That is because pistachio production in Rafsanjan is dependent on a lot of variables many of which are not measurable like sanctions, wet years and dry years, level of government control over wells' exploitation and water trade-off, the use of different kinds of fertilizer and pesticides combined with different farmers' agriculture/irrigation adaptation strategies to keep their annual production (Mehryar et al., 2015, Mehryar et al., 2016). So in this case the overall change of annual pistachio production does not correlate with a single variable change and cannot be presented by correlations between time-series. Rather, a network of perceived interactions explains the change of the pistachio production better. Therefore we used the P-FCM values for these three connection values in building DP-FCM. 
Besides, the FP-FCM connections' weights have also been compared with DFCM to investigate similarities and differences of farmers' perceptions with real data comparing to policy makers' perception (appendix $7 \&$ table 4). Overall, out of total 26 connections, 13 connections $(50 \%)$ of all connections are similar (with difference of $<0.3$ ); comparing data and policy makers 6 connections $(20 \%)$ are different (with difference of $\geq 0.3$ ), while comparing data and farmers 13 connections (50\%) are different. All connections which are similar comparing farmers and policy makers' perceptions are also similar to the available data. This means that policy makers' perceptions and knowledge of the SES reflect more closely the objectively measured-data than does the farmers' perceptions P-FCM (figure 5).
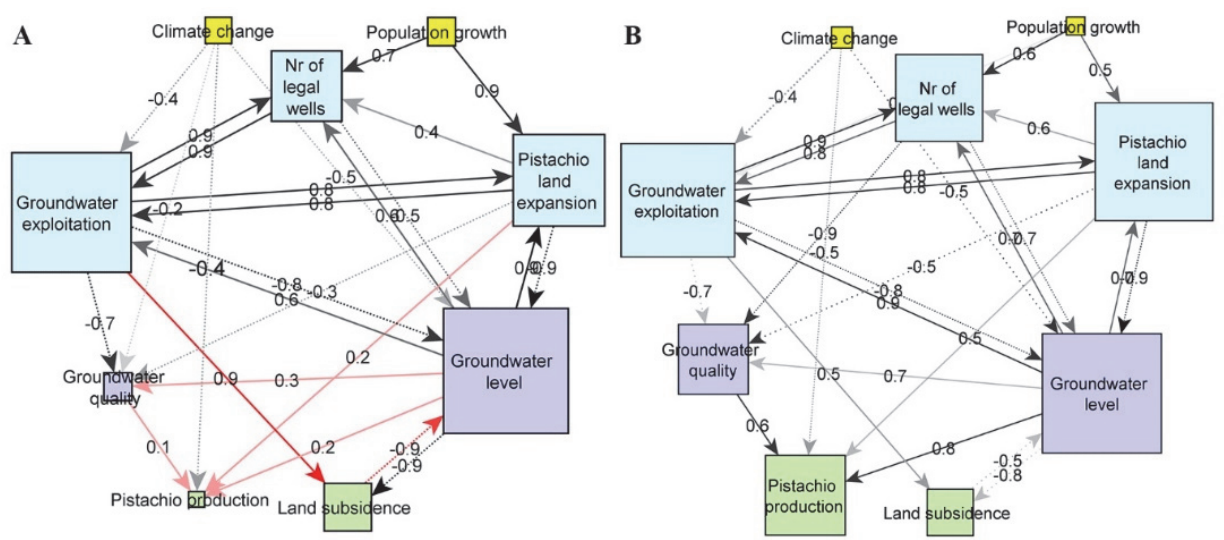

Figure 4.4: Graphical representation of (A) D-FCM (as in Fig. 3) and (B) PP-FCM with only common concepts and connections with D-FCM. The red lines in D-FCM represent the connections with significant different values from PP-FCMs. 
Table 4.3: Connections' weights in D-FCM, PP-FCM, and FP-FCM. Connections with similar values in the D-FCM, PP-FCM, and FP-FCM are shown in black; connections with different value between the D-FCM and PP-FCM are shown in red; connections with different value between the D-FCMs and FP-FCM are shown in blue.

\begin{tabular}{|c|c|c|c|c|}
\hline & Connections & $\begin{array}{l}\text { Measured } \\
\text { data } \\
\text { correlation }\end{array}$ & $\begin{array}{l}\text { Perceived- } \\
\text { Policy } \\
\text { makers }\end{array}$ & $\begin{array}{l}\text { Perceived- } \\
\text { farmers }\end{array}$ \\
\hline A & Population growth $\rightarrow \mathrm{Nr}$ of legal wells & 0.7 & 0.6 & 0.9 \\
\hline B & $\begin{array}{l}\text { Climate change } \rightarrow \text { Groundwater } \\
\text { exploitation }\end{array}$ & -0.4 & -0.4 & -0.1 \\
\hline C & Climate change $\rightarrow$ Pistachio production & -0.4 & -0.5 & -0.8 \\
\hline D & Climate change $\rightarrow$ Groundwater level & -0.5 & -0.5 & -0.4 \\
\hline $\mathbf{E}$ & Climate change $\rightarrow$ Groundwater quality & -0.2 & 0 & -0.9 \\
\hline $\mathbf{F}$ & $\begin{array}{l}\text { Pistachio land expansion } \rightarrow \\
\text { Groundwater overexploitation }\end{array}$ & 0.8 & 0.8 & 0.7 \\
\hline G & $\begin{array}{l}\text { Pistachio land expansion } \rightarrow \\
\text { Groundwater level }\end{array}$ & -0.9 & -0.9 & -0.6 \\
\hline $\mathbf{H}$ & $\begin{array}{l}\text { Pistachio land expansion } \rightarrow \\
\text { Groundwater quality }\end{array}$ & -0.3 & -0.5 & -0.9 \\
\hline $\mathbf{I}$ & $\mathrm{Nr}$ of legal wells $\rightarrow$ Groundwater level & -0.5 & -0.7 & -0.9 \\
\hline J & $\begin{array}{l}\text { Groundwater exploitation } \rightarrow \mathrm{Nr} \text { of legal } \\
\text { wells }\end{array}$ & 0.9 & 0.9 & 0 \\
\hline $\mathbf{K}$ & $\begin{array}{l}\text { Groundwater exploitation } \rightarrow \text { Pistachio } \\
\text { land expansion }\end{array}$ & 0.8 & 0.8 & 0.7 \\
\hline $\mathbf{L}$ & $\begin{array}{l}\text { Groundwater exploitation } \rightarrow \\
\text { Groundwater level }\end{array}$ & -0.8 & -0.8 & -0.9 \\
\hline $\mathbf{M}$ & $\begin{array}{l}\text { Groundwater exploitation } \rightarrow \\
\text { Groundwater quality }\end{array}$ & -0.7 & -0.7 & -0.8 \\
\hline $\mathbf{N}$ & $\begin{array}{l}\text { Groundwater exploitation } \rightarrow \text { Land } \\
\text { subsidence }\end{array}$ & 0.9 & 0.5 & 0 \\
\hline $\mathbf{0}$ & Groundwater level $\rightarrow \mathrm{Nr}$ of legal wells & 0.6 & 0.7 & 0 \\
\hline $\mathbf{P}$ & $\begin{array}{l}\text { Groundwater level } \rightarrow \text { Groundwater } \\
\text { exploitation }\end{array}$ & 0.6 & 0.9 & 0 \\
\hline $\mathbf{Q}$ & Land subsidence $\rightarrow$ Groundwater level & -0.9 & -0.5 & 0 \\
\hline $\mathbf{R}$ & $\begin{array}{l}\mathrm{Nr} \text { of legal wells } \rightarrow \text { Groundwater } \\
\text { overexploitation }\end{array}$ & 0.9 & 0.8 & 0.9 \\
\hline $\mathbf{S}$ & $\begin{array}{l}\text { Groundwater level } \rightarrow \text { Groundwater } \\
\text { quality }\end{array}$ & 0.3 & 0.7 & 0.6 \\
\hline $\mathbf{T}$ & $\begin{array}{l}\text { Pistachio land expansion } \rightarrow \mathrm{Nr} \text { of legal } \\
\text { wells }\end{array}$ & 0.4 & 0.6 & 0.8 \\
\hline $\mathbf{U}$ & $\begin{array}{l}\text { Pistachio land expansion } \rightarrow \text { Pistachio } \\
\text { production }\end{array}$ & 0.2 & 0.5 & 0.7 \\
\hline $\mathbf{v}$ & $\begin{array}{l}\text { Groundwater level } \rightarrow \text { Pistachio land } \\
\text { expansion }\end{array}$ & 0.9 & 0.7 & 0.6 \\
\hline $\mathbf{W}$ & Groundwater level $\rightarrow$ Land subsidence & -0.9 & -0.8 & -0.8 \\
\hline $\mathbf{x}$ & $\begin{array}{l}\text { Groundwater level } \rightarrow \text { Pistachio } \\
\text { production }\end{array}$ & 0.2 & 0.8 & 0.6 \\
\hline $\mathbf{Y}$ & $\begin{array}{l}\text { Groundwater quality } \rightarrow \text { Pistachio } \\
\text { production }\end{array}$ & 0.1 & 0.6 & 0.6 \\
\hline $\mathbf{Z}$ & $\begin{array}{l}\text { Population growth } \rightarrow \text { Pistachio land } \\
\text { expansion }\end{array}$ & 0.4 & 0.5 & 0.3 \\
\hline
\end{tabular}




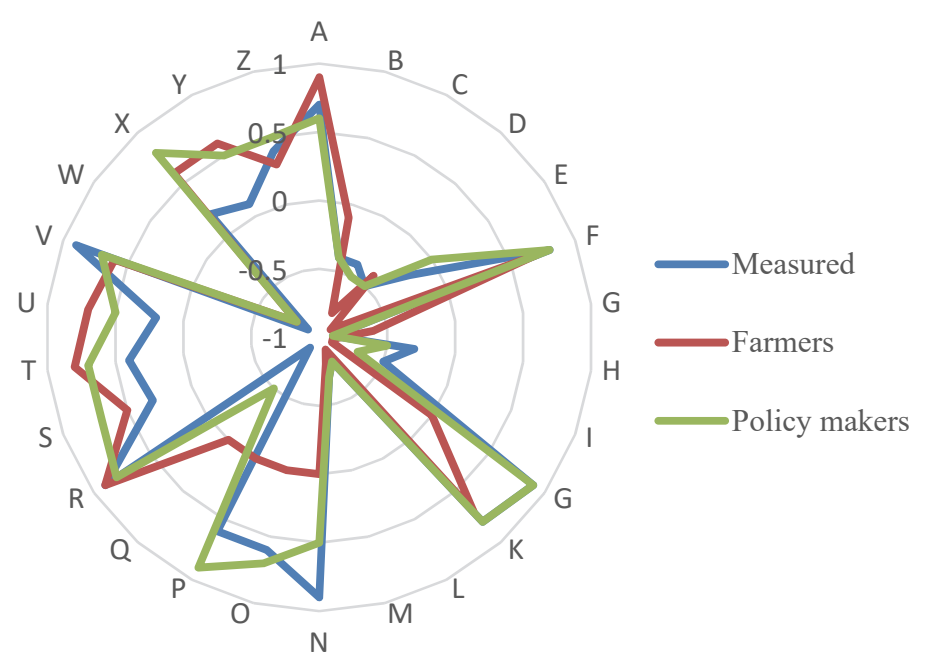

Figure 4.5: Values of connections in three different FCMs: D-FCM, PP-FCM, FP-FCM.

Finally, the validated connection values are added to the M-FCM i.e. being replaced by perceived connection values. Visual representation of the resulting $\mathrm{M}-\mathrm{FCM}$ is shown in figure 6. It comprises nine measured-concepts, and twentyfour perceived-concepts, twenty-three measured connection weights and ninety-eight perceived connection weights. The M-FCM is more influenced by farmers' perceptions than by objective data. Consequently, the differences between the nodes' sizes of M-FCM and the P-FCM may not be easily recognized but by running both FCM models and showing the models' evolution over time, the effect of the differences on system behaviour can be observed and analysed (section 3.2). 


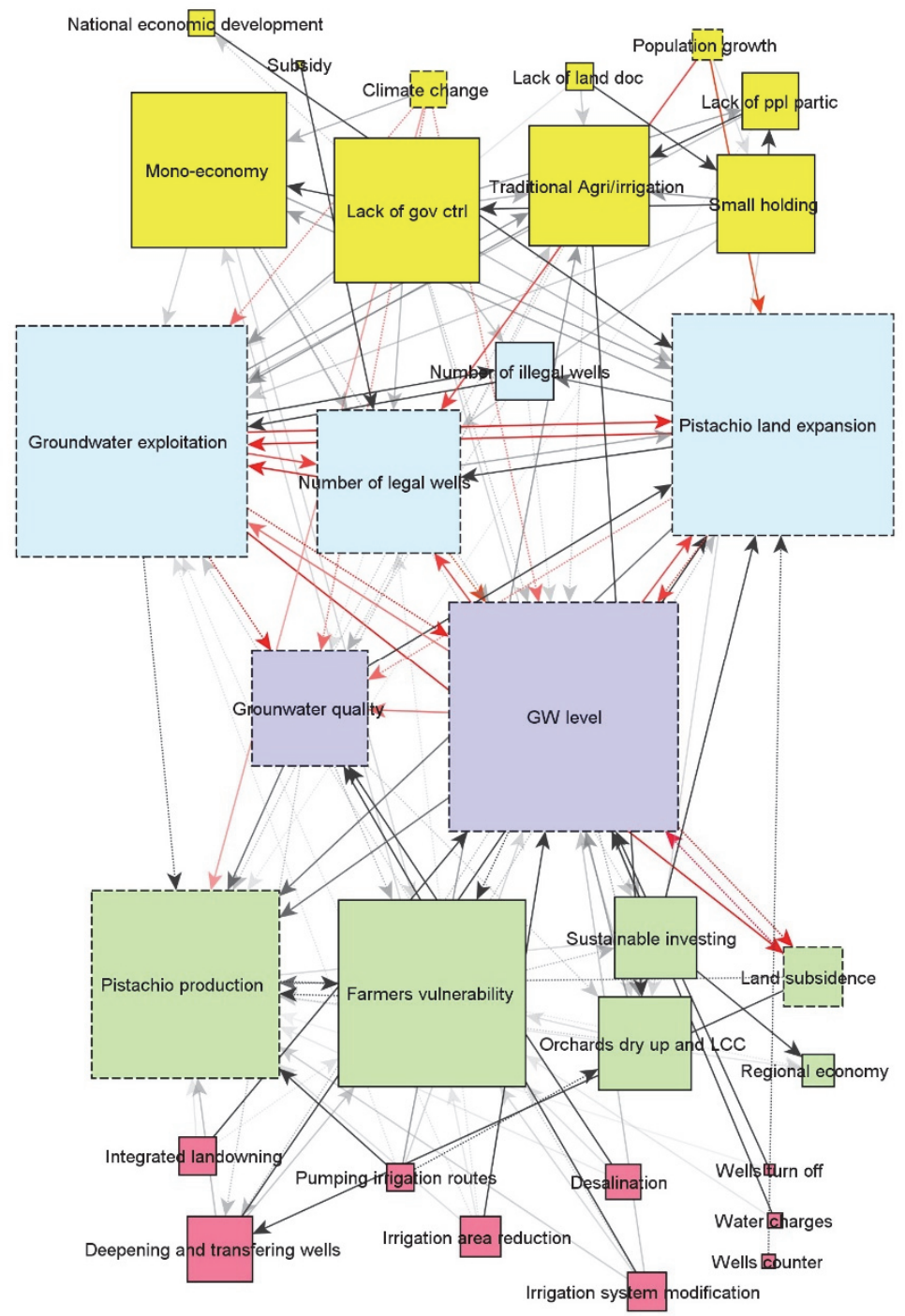

Figure 4.6: M-FCM, Size of the node indicates the centrality (importance) of variables. Red connections represent the data-driven connection value; black connections represent perceived connection value. Solid boxes represent concepts influencing and being influenced by perceived connections and dashed boxes represent concepts influencing and being influenced by both data-driven and perceived connections. There are no concepts that are only influenced by data-driven connections.

\subsubsection{Policy option simulation with M-FCM model}

With the M-FCM model, each of the four policy options have been run and the steady state of each option was compared with the baseline steady state. Figure 7 indicates the positive and negative changes of various concepts for each policy option. Concepts without change are not shown. 
Policy option 1: By far the economic change policy, which aims at reinforcing economic diversification instead of agriculture-dependent economy, has the largest influence on increasing groundwater level. It also has the largest impact on decreasing groundwater exploitation, number of illegal wells, farmers' vulnerability, land subsidence, and increasing regional economy and sustainable investment of the region. This policy option appears to have the most recovering impact on the system.

Policy option 2: The irrigation and agriculture change policy has a significantly larger influence on increasing pistachio production and pistachio land expansion and decreasing pistachio land transfer, and a slightly larger influence on increasing groundwater quality and regional economy compared to the other policies.

Policy option 3: Empowering peoples' participation in water resource management has the largest impact on decreasing the usage of traditional and inefficient irrigation and agriculture systems and overcomes the lack of government control and monitoring of groundwater in the region. Nevertheless, it has the lowest impact on increasing groundwater level among all options.

Policy option 4: Government control and monitoring policy has a significant influence on increasing national economic development and decreasing the number of illegal wells, but it has a much larger influence on declining peoples' participation too.

All 4 policy options have equally little impact on groundwater overexploitation, number of legal wells, land subsidence, and deepening wells, which means these variables have been ignored or paid minimal attention by all the policy options. Whereas, pistachio production, orchards dry up, regional economy and sustainable investment are the concepts on which two or more policies have large impact, which means these concepts receive the most attention from multiple policies. There is no concept on which all 4 policy options have equally high impact. Moreover, two concepts i.e. lack of people participation and pistachio land expansion show a different direction between policy options' impacts. Policy of government control increases lack of people participation in contrast to other policies that either decrease or have no impact on lack of people participation. Likewise, policy of irrigation system change increases pistachio land expansion, versus other policy options that either decrease or have no specific impact on pistachio land expansion. 


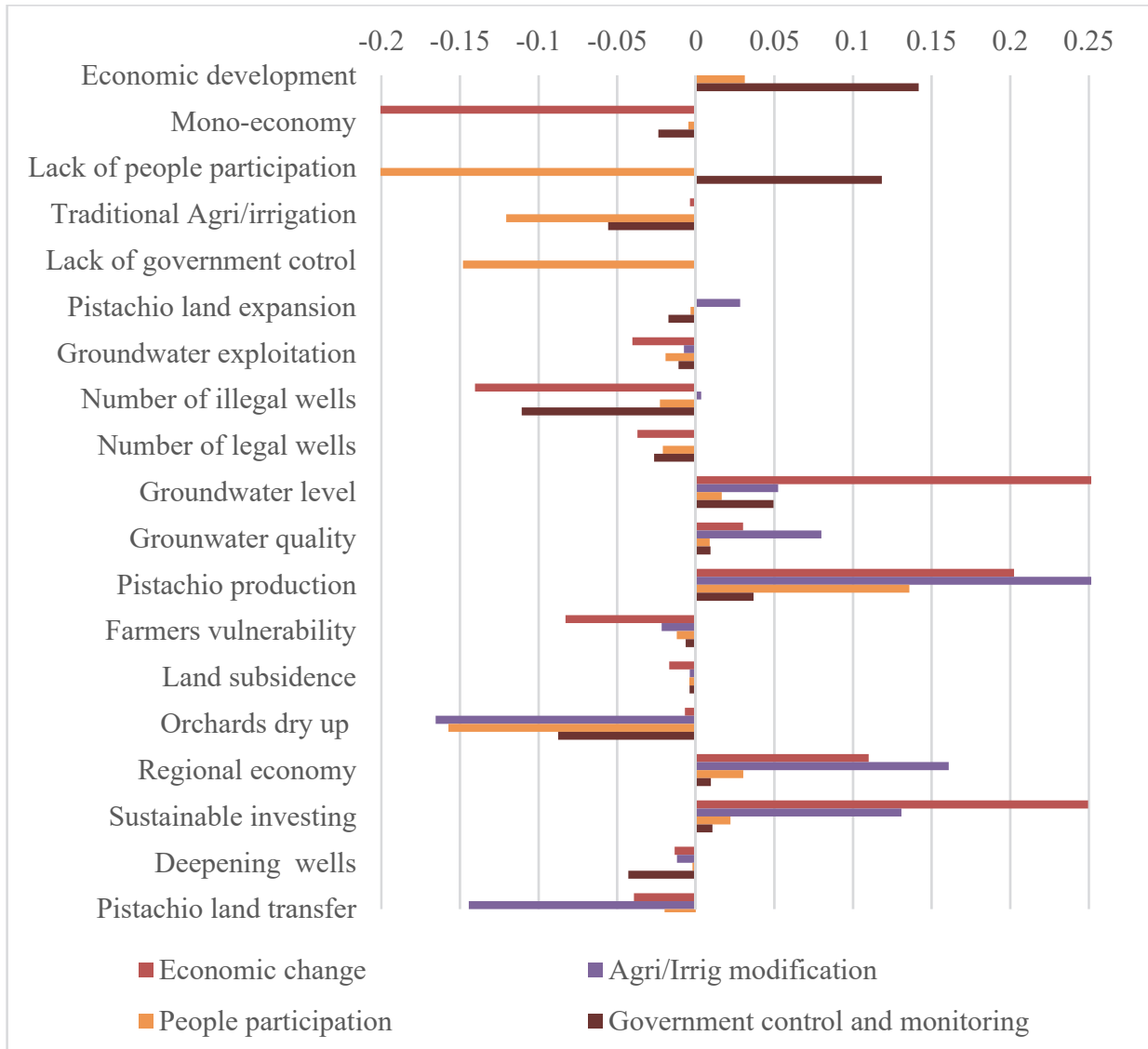

Figure 4.7: Results of four policy options simulation in M-FCM model and their impact on each indicator compared to the baseline steady state.

\subsubsection{Comparison of M-FCM and CP-FCM simulations}

Finally, we compare the simulation results of the M-FCM with the CP-FCM. The main question here is: to what extent does the new M-FCM model produce different simulation results from the standard P-FCM model?

Comparing the simulations shows that only for the first two policy options, i.e. economic growth and irrigation/agriculture system modification, the results for the M-FCM and the CP-FCM are substantially different. Yet, the last two policy options i.e. people participations and government control produce very similar simulation result for both M-FCM and CP-FCM (figure 8). This is because the first two policy options manipulate both data-driven and perceived concepts whereas the last two manipulate only perceived concepts. For example, in policy option 1) economic change, the two data-driven concepts of pistachio land expansion and pistachio production and one perceived concepts of monoeconomy were clamped. Yet, in policy option 3) peoples' participation and 
integrated landownership, all clamped concepts are perceived-concepts i.e. lack of peoples' participation, small holding, community-based organization (table 3 ). More details of the policy simulation comparisons are as follows:

Policy option 1 (clamping data-driven and perceived concepts): In both M-FCM and CP-FCM the influence of economic change on increasing groundwater level was the largest among all policies. However, overall the CP-FCM showed lower impact than the M-FCM, and for some variables with significant changes in the $M-F C M$ simulation, the changes in CP-FCM were not significant (compare variables with no-significant value of CP-FCM with the same variables value of M-FCM in figure 8).

Policy option 2 (clamping data-driven and perceived concepts): the M-FCM simulation showed overall a more positive impact of the agriculture and irrigation system change option than the CP-FCM. In the CP-FCM this policy has the largest impact on increasing pistachio production and decreasing pistachio land transfer but its positive impact on groundwater level is the least among all policies. Whereas in the M-FCM this policy has the largest impact on increasing pistachio land expansion, pistachio production, groundwater quality and regional economy and decreasing orchards dry up and pistachio land transfer and the second largest impact on increasing groundwater level.

Policy option 3 (clamping perceived-concepts): In both the M-FCM and the CPFCM models, peoples' participation policy option has the largest impact on reducing usage of traditional irrigation or agriculture systems as well as lack of government control. The impact of this policy option on other concepts is similar for both models.

Policy option 4 (clamping perceived-concepts): In both the M-FCM and the CPFCM models the government control and monitoring policy option results in a significant, large increase in national economic development, as well as decreasing the mono-economy of the region, peoples' participation and deepening wells. The other concepts show similar impacts for both M-FCM and CP-FCM simulations.

Therefore, M-FCM and CP-FCM in our sample produced different results in those simulations that have a direct impact on data-driven concepts i.e. concepts influenced by both data-driven and perceived connections. The two policy options with direct impact on perceived concepts -i.e. only influenced by perceived connections - have very similar results. Though this sample for making a general result is small, but the result validates the consolidation of perceived concepts' behaviour in the model while adding new data-driven concepts to the model. In other words, by adding objective causal relations: 1) the stakeholders' perception and knowledge are maintained for the 
subjective part of the model, and 2) the model behaviour is expected to be closer to reality because of the inclusion of objective, measured data next to the subjective data.

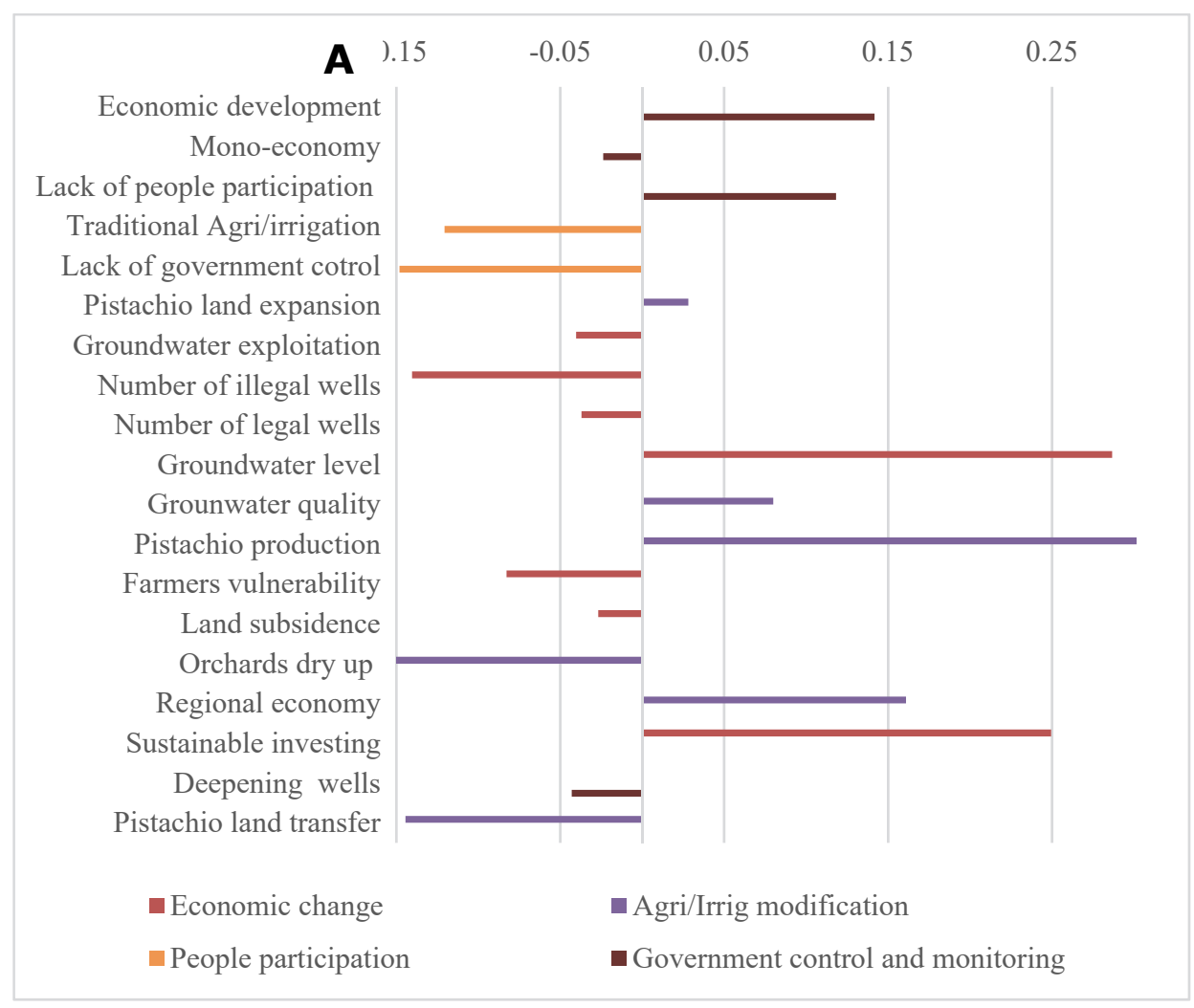




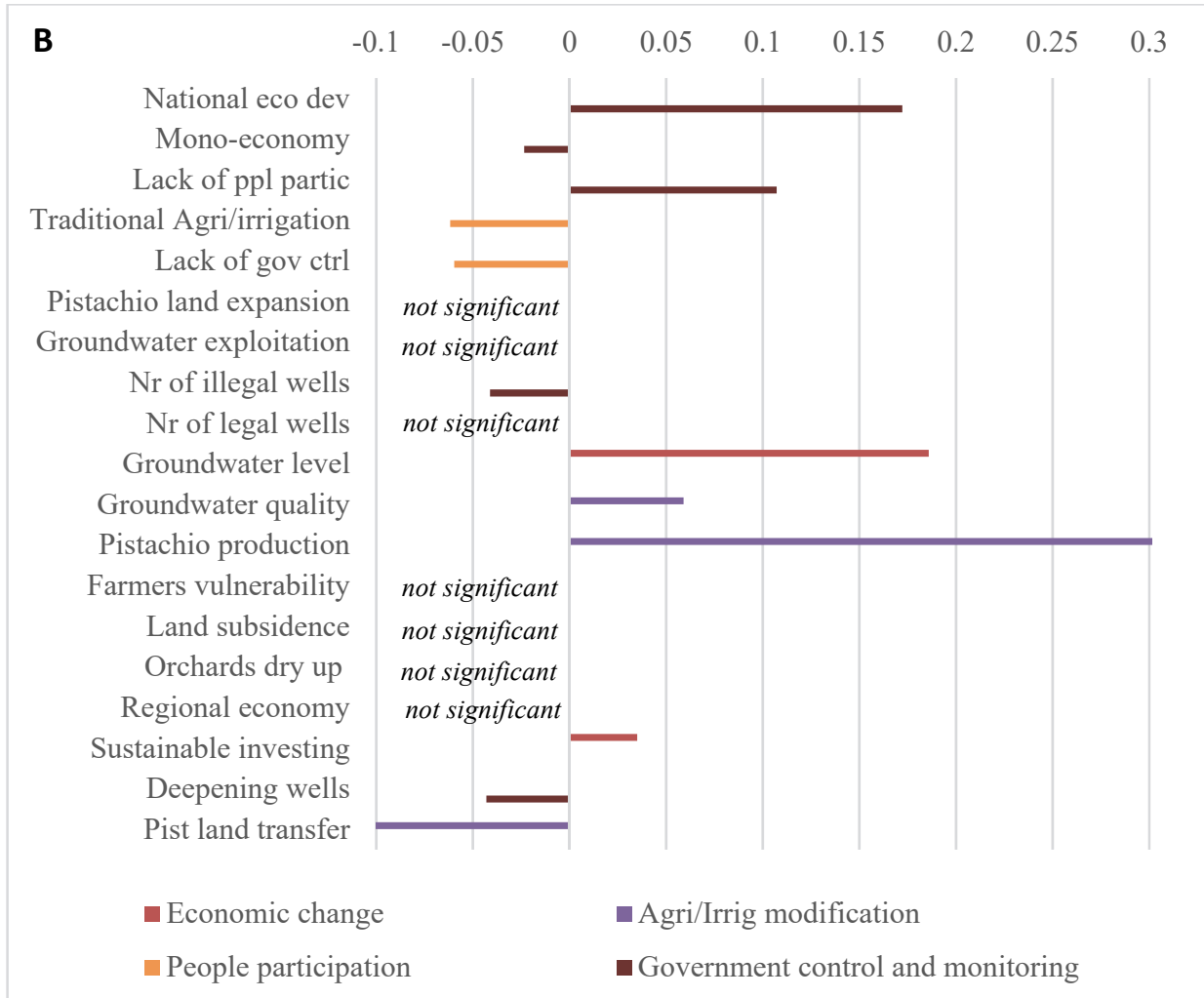

Figure 4.8: Result of policy option simulation in A) M-FCM and B) CP-FCM models. For each variable, the policy option with largest impact is shown. Impacts $<0.02$ are considered as not significant.

\subsection{Discussion and conclusion}

Policy making in SES sustainability challenges requires a deeper understanding of complex, multi-variables and multi-behaviour systems. Lack of empirical data about social variables, on one hand, and a potential lack of accuracy in public perception about hidden and slow-changing variables, on another hand, call the necessity for integrating both subjective and objective knowledge in modelling SESs for policy-making purposes. This paper intended to introduce and analyse the potential of a new approach in FCM method to incorporate both stakeholders' perceptions and objective data into a SES model and to investigate to what extent the combination of these two data sources improves the quality of a model using only perception data.

The results of this study showed that combining farmers' perceptions and objective data in the case of water scarcity in Rafsanjan could model and represent the complex SES of the farming community best. Both subjective and objective knowledge could overcome limitations caused by scarcity of 
formal knowledge to model the socio-economic part of the system - e.g. impact of subsidy and small holding on groundwater use and farmers' vulnerabilityand the inability of perceived knowledge to model some ecological parts of the system - e.g. lands subsidence, groundwater level, and groundwater quality-.

The comparison between M-FCM and CP-FCM simulations indicated that simulation of policy options that have direct influence on data-driven variables have substantially different results in M-FCM from those of a CP-FCM. In this type of policy options, the behaviour of the M-FCM is expected to be closer to reality than the $\mathrm{CP}-\mathrm{FCM}$ due to the integration of accurate, quantitative data in the model. However, this result requires further studies on application of $M$ FCM method for different kind of policy options.

The comparison between the connection weights of D-FCM, PP-FCM and FPFCM showed that policy makers' perceptions are generally closer to measureddata than farmers' perceptions and in many cases, farmers' perceptions have large difference with both data and policy makers' perceptions. Among the common connections between these three maps, $20 \%$ have significant value difference between D-FCM and PP-FCM, 50\% have significant value difference between FP-FCM and D-FCM, and $40 \%$ have significant value difference between FP-FCM and PP-FCM. This difference represents the big knowledge gap between farmers and policy makers/ researchers whose communication and collaboration is complementary for a proper understanding of the system.

Using two source of quantitative and qualitative knowledge to validate the results of model is a strong point of the proposed method. During our analysis, we found the experts knowledge is a necessary element in validating the FCM built on time-series data. Yet, this can be also done through a workshop meeting to discuss the results after building D-FCM. However, in this case the conflicts of perceptions and knowledge among experts from different departments should be managed.

\subsubsection{Method's application}

To apply the method properly, it is suggested to use the three steps in this study in the following order: 1) collecting stakeholders' perceptions and building the whole system with P-FCM; 2) defining data-driven concepts and connections; 3 ) replacing concepts and weights in the P-FCM for which datadriven information is available, thereby building the D-FCM into the P-FCM. This approach has three advantages:

1) An FCM made by a large number of stakeholders has already (subjectively) verified causal relations. The causality of these relationships cannot be verified by correlations among concepts. Thereby, the already verified causal relationships by stakeholders can later be used in D-FCM. 
2) In a M-FCM, even the concepts recognized to represent data-driven variables can be influenced by both data-driven and perceived-connections. Yet, by creating the D-FCM and P-FCM separately and combining them together, one might miss the interlinked relations between measured and perceived concepts. Having the P-FCM of the whole system first and then extracting D-FCM from it helps to keep such interconnections.

3) Knowing stakeholders' perceptions about the system might help to find out the reliable source of data in case of confusing or conflicting data. For instance, in our study, we realized that farmers debate the accuracy of pistachio production figures reported by the government or international organizations like FAO, so we used data from a pistachio production report of the Iranian Pistachio Association, a cooperative non-profit organization that is in close cooperation with local farmers.

\subsubsection{Limitations of method}

The arguably nature of some concepts whether they can be better presented by stakeholders' perceptions or empirical data might be a limitation of this method. However, the assumption of this paper is that for developing M-FCM a former understanding and recognition of the perception-based and datadriven variables of the system are available. Different variables may have different nature and behaviour in different contexts. Therefore, the question that which kind of data is more appropriate for each variable is a case-specific issue and require a typology for the measurable variables and perceptible variables in each specific case study.

Another weak point of this method has been the common limitation of FCM applications to be spatially explicit. In our case study the groundwater quality variable, for instance, has particularly shown different impacts on other variables in different locations of Rafsanjan. However, this differences are not represented in our FCM models, rather an average of impacts are used. Thus, in the case studies, that spatial heterogeneity is important to be considered in policy option analysis (which was not the case of this study), different FCMs should be developed for different locations.

\subsubsection{Non-applicability of method}

It is important to note that the M-FCM is not the preferred model in all cases and that a standard FCM with solely reliance on stakeholders' perception might be sufficient for some SES modelling. These cases can be categorized as: 1) studies that aim to capture only different stakeholder perceptions of a problem (Giordano et al., 2007, Matmir et al., 2017), or to compare these (Gray et al., 2012) or to show to policy makers how local stakeholders perceive a problem (Reckien et al., 2011, Reckien et al., 2013), 2) studies addressing more social 
or perception or belief related issues, like social vulnerability to climate change (Reckien, 2014) or farmers' adaptation strategies (Kulsum et al., 2017) where stakeholders have the best knowledge of the system, 3) studies where reliable measured-data are lacking and stakeholders' perceptions are the only data source.

Building FCM with sole reliance on time-series data, e.g. FCM learning methods, can also be sufficient in the cases where rich data-sets for all variables of the system is available, which is not the case of social-ecological and socialtechnical systems where peoples' behaviour has an important role.

\subsubsection{Future studies}

The aim of this study was to demonstrate the construction of a M-FCM in a SES for policy option analysis. We could validate the D-FCM by comparing it with policy makers' knowledge via triangulation. Yet, the results of this study i.e. the impacts of different policy options over a long-time horizon, cannot be validated with current data and knowledge. Validation can only be done after implementation of one of the policies and by comparing the impacts of the chosen policy with those of the no-policy situation.

Besides, future studies are needed to strengthen the link between the D-FCM and P-FCM. Although this study shows how to develop and combine two FCMs that are built using data and perceptions, a methodology that directly provides input from both measured, time-series data and perceived data in FCM construction can be even more valuable. 


\section{Chapter 5*}

\section{From Individual Fuzzy Cognitive Maps to Agent Based Models: Modelling Multi- Factorial and Multi-stakeholder Decision- Making for Water Scarcity}

\footnotetext{
* This Chapter is based on:

Mehryar, S., Sliuzas, R.V., Schwarz, N., Sharifi, A. and van Maarseveen, M.F.A.M.,

2018. From Individual Fuzzy Cognitive Maps to Agent Based Models: Modelling MultiFactorial and Multi-stakeholders for Decision-Making in Water Scarcity. Journal of Environmental Management (manuscript under review).
} 


\begin{abstract}
Policy making for complex Social-Ecological Systems (SESs) is a multi-factorial and multi-stakeholder decision making process. Therefore, proper policy simulation in a SES should consider both the complex behaviour of the system and the multi-stakeholders' interventions into the system, which requires integrated methodological approaches. In this study, we present an integrated modelling methodology combining an Agent Based Model (ABM) with Fuzzy Cognitive Mapping (FCM) to simulate impacts of policy options in a severe water scarcity situation. First, the relations among environmental variables and behavioural rules of stakeholders are captured with FCMs that are developed with both qualitative and quantitative data, i.e. stakeholders' knowledge and empirical data from studies. Then, an ABM is developed to simulate the interactions of stakeholders and their impacts on environmental variables in a case study of a farming community facing water scarcity in Rafsanjan, Iran. Our approach covers four main aspects that are crucial for policy simulation purposes: 1) causal relationships, 2) feedback mechanisms, 3) social-spatial heterogeneity and 4) temporal dynamics. Therefore, this approach is particularly useful for ex-ante policy options analysis. We simulate the impacts of different policy options compared to a baseline scenario. The results suggest that a policy of facilitating farmers' participation in management and control of their groundwater use leads to the highest reduction of groundwater use and would help to secure farmers' activities in Rafsanjan.
\end{abstract}

Keywords: Social-ecological systems; Fuzzy cognitive mapping; Agent-based modelling; Policy option analysis; Water scarcity. 


\subsection{Introduction}

Environmental management and policy making for complex Social-Ecological Systems (SESs) are multi-factorial and multi-stakeholder decision-making processes. This has two important implications. First, SESs include multiple, interacting social and ecological factors (variables), e.g. natural resources, climate change, human interventions, emigration and social vulnerability. Interactions between these factors influence the behavior of the whole system. Therefore, policy analysis methods for SESs should be able to simulate the exante impact of policies by considering the dynamic behavior and interactions of all important factors. Second, SESs involve many different stakeholders, from resource consumers to policy makers and managers, all of whom have different interests, which sometimes leads to conflicting decisions and actions. This heterogeneity may change the impact of policy options in different contexts (Levin et al., 2013, Mease et al., 2018). To consider these two aspects of multi-factorial and multi-stakeholder decision-making, two approaches for simulating the impacts of policy options in SES have been developed. A factorbased approach that represents changes in factors (variables) of a system and their interactions (Macy and Willer, 2002), e.g., Fuzzy Cognitive Mapping (FCM) (Kosko, 1986a). An actor-based approach that represents decisions, behaviors and interactions of stakeholders, e.g., Agent-Based Modelling (ABM) (Gilbert, 2008).

\subsubsection{Fuzzy Cognitive Mapping}

FCM, a combination of fuzzy logic and cognitive mapping, is widely used in environmental management and SES studies to represent knowledge of systems under conditions of data scarcity and data uncertainty (Özesmi and Özesmi, 2004, Papageorgiou and Kontogianni, 2012, Reckien, 2014). Structurally, it consists of a set of nodes (concepts) and fuzzy signed directed edges (connections) representing the strength of the causal relationships between concepts (Kosko, 1986a). Thus, it encodes multiple causal relationships between variables of a system. FCM models are usually developed with a participatory approach. Stakeholders who are familiar with the operation and behavior of a system or specific problem of a system are asked to mention the most important concepts (environmental, social, ecological or economic variables), their causal relations, and the weights of the connections (i.e. how much a change of one concept causes a change in another concept) (Özesmi and Özesmi, 2004). A range of individual mental models of stakeholders is developed and aggregated into a semi-quantitative and standardized FCM model for simulation (Mehryar et al., 2017, Vasslides and Jensen, 2016). Thus, the connections in participatory FCMs represent perceived causality determined via crowdsourcing. 
FCM uses individuals as the units of data collection and analysis but aggregates their knowledge to provide a macro-level view of an entire system's behavior. Thus, FCM does not represent individuals' dynamic interactions with their environment. Besides, FCM provides semi-quantitative output data from qualitative stakeholders' knowledge, which may be used in combination with mathematical models. Therefore, FCMs are potentially useful in modelling aggregate human behavior and decisions (An, 2012). However, their lack of stakeholders' interactions, as well as temporal and spatial explicitness are their main limitations.

\subsubsection{Agent Based Modelling}

$A B M$ provides a micro-level view of a system since each agent is explicitly represented and interacts with other agents as well as with the environment (Giabbanelli et al., 2017). Typically, ABMs are spatially explicit and simulate dynamics over time, which makes them appealing to model SESs. However, ABMs face the challenge of acquiring data for describing: 1) agents' behavioral options, 2) decision-making processes (the way an agent makes decisions), and 3) decision outcomes (impacts of their actions on others and on the environment). Due to the complexity of human decisions and actions, ABM studies regularly rely on rational choice theory to describe agents' behavior (Schlüter et al., 2017, Groeneveld et al., 2017). However, actual human behavior is subjective and has bounded rationality due to limitations of information access, time, personal beliefs and perceptions (Elsawah et al., 2015). This is particularly important in models for policy support (Schlüter et al., 2017). As a result, many modelers using ABMs try to replicate actual human behaviors and decision-making as closely as possible (Filatova et al., 2013) via participatory methods (An, 2012) such as role-playing games (Bousquet et al., 2002, Castella et al., 2005), Bayesian belief networks (Sun and Müller, 2013), cognitive mapping (Elsawah et al., 2015) or ethnographic methods (Ghorbani et al., 2015). Yet, the formulation and parametrization of qualitative knowledge gained through such approaches, their combination with quantitative data, and the identification and calibration of causal feedback mechanisms of a SES remain key challenges (Robinson et al., 2007, Sun and Müller, 2013, Ghorbani et al., 2015, Venkatramanan et al., 2017).

\subsubsection{Combining FCM and ABM}

FCM and ABM are complementary in supporting SES policy making. Surprisingly, there have been only a few attempts to combine these two methods for SES modelling. Two studies have suggested distinct approaches to combine FCM and ABM. Elsawah et al. (2015) proposed a methodology that developed cognitive maps for use in ABM development. More specifically, they used cognitive maps to translate the subjective qualitative description of decision-making into formal rules in the ABM. In contrast, Giabbanelli et al. 
(2017) proposed two options for creating hybrid models, in which FCM and $A B M$ are coupled and co-exist over a model run. In one option, an $A B M$ represents the mental model of each agent as an FCM that can change through interactions with other agents. In another option, selected parts of an FCM are informed by an ABM. To our knowledge, no study has yet reported on implementing a combination of an FCM and an ABM such that the FCM informs both the agents' behavioral rules at the micro-level and the humanenvironment interaction rules at the macro-level. This is where our study steps in. Using the example of water management in Rafsanjan Township, Iran, we explain how we used FCMs to conceptualize an actor-based ABM. This ABM allows for testing the effects of different policy options and thus enables us to investigate dynamic processes and interactions among agents; a process which an FCM alone cannot do.

Similar to Elsawah et al. (2015), our focus is on structuring and using the collected qualitative data from a set of FCMs to develop an ABM. Yet, our approach significantly differs in two ways from theirs. First, we use FCMs instead of cognitive maps. Second, we use FCMs to model the whole system, including and not limited to stakeholders' actions. Thus, the FCM provides a macro-level view of the system i.e., the perceived interactions between social, ecological, environmental and economic variables, and also provides information for micro-level decision-making of agents i.e., type of actions and impacts of actions on the environment. The outcome of our proposed modelling framework is useful for ex-ante policy options analysis.

This paper is organized as follows. Section 2 introduces our step-wise methodology and presents a case study. Section 3 describes the implementation of the methodology in the case study, and then in section 4, the results of the policy simulation in the case study are presented and discussed. Sections 5 and 6 reflect on the final results and the methodology and conclude.

\subsection{Methodology}

Our methodology consists of three main steps (Figure 1): 1. FCM modelling, 2. Translating FCM to ABM, and 3. ABM implementation and assessment. In step 1 , the individual maps are first collected by interviewing stakeholders (step 1.1). Then, the individual maps are merged to create one FCM for each specific group of stakeholders (step 1.2). Finally, the time-series data is added to these subjective group FCMs to create the subjective-objective FCMs (step 1.3). In step 2, first the Overview, Design concepts, and Details (ODD) protocol is used to define the main elements required for ABM development in this study. Then, a Condition-Action-Impact (CAI) diagram is introduced and developed to translate and categorize the FCMs' concepts into the set of available actions, 
and conditions-impacts for each action. Finally, a UML activity diagram is used to represent the sequential steps of actions and spatial-temporal aspects of decision-making processes by using the outcome of the CAI diagrams. In step 3 , the ABM model is simulated and the results are validated with the historical data. The validated $A B M$ is used to simulate the possible impacts of policy options via "what-if" analysis and compare their results with those of the baseline scenario. Finally, a sensitivity analysis is applied to the parameters of the model.

In the following sub-sections, each of these steps is discussed in more detail.

\section{STEP 1: FCM Modeling}

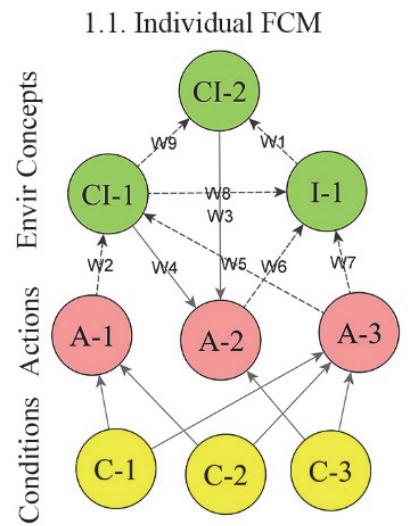

1.2. Group specific FCMs

1.3. Subjective-Objective FCMs
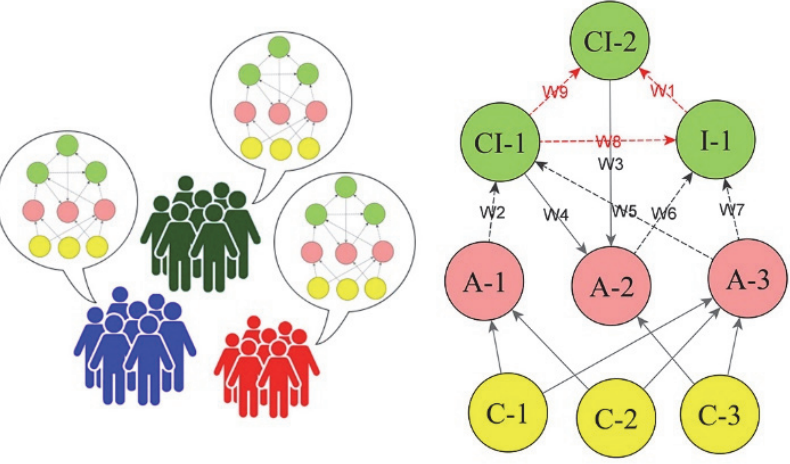

STEP 2: Translating FCM to ABM

2.1. ODD protocol

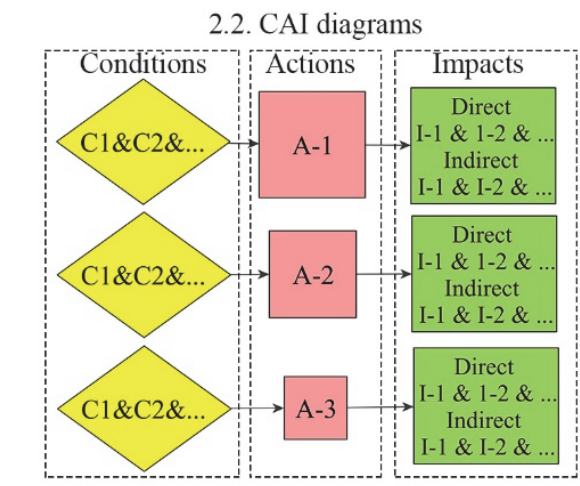

2.3. UML diagrams

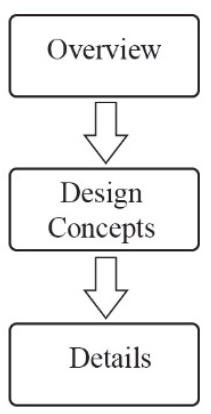

STEP 3: ABM implementation and assessment

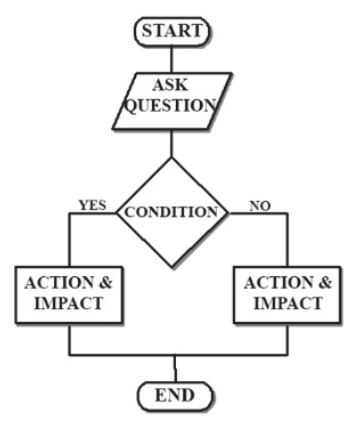
3.1. Coding
3.2. Validation
3.3. Policy simulation
3.4. Sensitivity analysis

Figure 5.1: Main steps and sub-steps of methodology. Coding scheme - A: Action, C: Condition, I: Impact, CAI: Condition-Action-Impact, UML: Unified Modeling Language. In FCMs: red connections: weighted based on objective data, black connections: weighted based on subjective data, dashed lines: impact connections, solid lines: driving connections. 


\subsection{Model building}

\subsubsection{Case study: water scarcity and farming community adaptation in Rafsanjan, Iran}

This study is a part of a research project that aims to support policy making in an SES of a farming community in Rafsanjan, Iran, which is facing severe water scarcity. This project uses participatory methods and techniques to involve and integrate stakeholders' knowledge, perceptions, decisions and actions in policy making under water scarcity conditions. Rafsanjan is among the top producers and exporters of pistachios in the world. Being in an arid and semi-arid region, pistachio farmers in Rafsanjan depend entirely on groundwater to irrigate their orchards, however, their production has been severely threatened by water scarcity in recent years (Mehryar et al., 2015, Mehryar et al., 2016).

Water scarcity in Rafsanjan is a tightly intertwined social-ecological problem. Many social and ecological variables are influencing or being influenced by water scarcity e.g. precipitation, groundwater use, pistachio production, land cover change, farmers' social-economic vulnerability, land subsidence, etc. Also, different groups of farmers with different interests and access to resources have various and sometimes conflicting reactions to water scarcity. The buying-out of small farmers by large-farmers, water marketing between small and large farmers, integrated farming and reducing orchard extents are among the famers' adaptive reactions to water scarcity. In policy option simulation for such an SES, the interactions between multiple variables and multiple stakeholders should be considered.

\subsubsection{Step 1: FCM modeling}

\section{Collecting individual maps}

There are different methods for individual FCMs' data collection e.g. extracting data from transcripts of interviews, remotely online mapping with stakeholders, and face-to-face semi-structured interviews that can be done via either individual or group discussions with stakeholders (Özesmi and Özesmi, 2004, Gray et al., 2014, Jetter and Kok, 2014). While all of these methods can be valid, different contexts may require specific methods. In this case study, due to the complexity of the water scarcity problem and the farmers' mistrust to share their information and perceptions, we chose to collect data with faceto-face interviews. These were useful in building a trustful relationship with interviewees, making the interview purpose explicit, and repeatedly offering explanations to the interviewees (Rahimi et al., 2018). Furthermore, due to the diversity of farmers in the area, and the complexities of their individual water problems, we chose individual interviews. In this way, we could capture the diverse, individual perceptions and local knowledge of farmers without them being influenced by larger, more powerful farmers (which could be the 
case in focused group discussion). Thus, we conducted individual interviews with 60 farmers in August-September 2015. All the interviews were done with in-depth, open-ended questions. Interviewees were selected to represent different farm sizes (large, medium and small), from different sub-regions of Rafsanjan (a sample of the oral consent script alongside the interview questions can be seen in appendix 10).

The interviews were led by two main questions and two sub-questions:

1. What have been the main causes and impacts of water scarcity in your region/farm?

1.1. How much has each of these variables caused an increase or decrease of other variables?

2. What have been your adaptive actions to combat water scarcity in your farm, and what have been the conditions to implement each action?

2.1. How much has each action impacted other variables mentioned earlier?

The interviewees were free to mention any concepts related to the questions 1 and 2: causes and impacts of water scarcity (e.g. precipitation, irrigation efficiency, agricultural productivity, economic situation, etc.), their adaptive actions (irrigation system change, deepening wells, integrated farming, etc.), and conditions of actions which could be a word or a phrase (e.g. having government loan for irrigation change, having permission for well's deepening, willingness of neighbor farmers for integrated farming, etc.). The concepts related to question 1 and 2 provided environmental concepts, and condition/action concepts, respectively (figure 1 , step 1.1).

The interviewees were also asked about the degree of influence of each variable (i.e. actions or environmental variables) on other variables (questions 1.1 and 1.2). They were asked to identify causal weights of relations based on the linguistic values of "very low", "low", "average", "high" and "very high". Such values were shown in the maps via a five points numerical scale $(0.1$, $0.3,0.5,0.7,0.9)$, corresponding to the five linguistic values. A positive value indicated that an increase in one concept caused an increase in another. A negative value indicated that an increase in one concept caused a decrease in another concept (Mehryar et al., 2017).

Regarding the second question, farmers were also asked to specify the frequency of each action, i.e., if the action is repeated every month, every year, etc. or taken only once (e.g. desalination). Moreover, farmers were asked about the situation that leads them to take each specific action, which could be not-changing variables. Therefore, the interviewer wrote down the fixed, i.e. true/false, conditions as input variables into the actions e.g. having documents or legal permission. For such variables, we used the structure of 
cognitive maps, i.e. including connections without weights where connection arrows represent implication and are interpreted as "may lead to" (Elsawah et al., 2015).

Important concepts and causal connections were drawn on the paper during the interviews by the researcher who constantly validated these with interviewees (an example from one of the interview maps can be seen in the appendix 12). The result of this step is many individual maps including the environmental network and actions of stakeholders. Each map is then stored as an adjacency matrix.

\section{Generating group specific FCMs}

To develop an FCM model, all of the individual maps are aggregated to a single unified model that encompasses all of the individual's knowledge. The individual maps are merged through matrix algebra, whereby each entry of the merged model is the average of the connection weights assigned by individuals (Vasslides and Jensen, 2017)-other approaches for group-level aggregation of FCMs are proposed in Gray et al. (2014) and Lavin et al. (2018). However, stakeholders may differ in their preferences, decisions and rules of behavior. By aggregating all individual maps, the heterogeneity of stakeholders is lost. To preserve the diversity of decision makers' mental models, the individual cognitive maps can be aggregated into different groups of FCMs. Categorizing FCMs can be based on the main drivers of differing stakeholders' decisions and actions. Researchers' insights, gained during the interviews and data analysis, provide a useful input for spotting the drivers of heterogeneous actions by stakeholders. For example, a researcher may notice that a specific socioeconomic or spatial characteristic like economic situation, location or resource access is influential. Then, the FCMs can be categorized and aggregated in different groups based on such a particular characteristic.

During the interviews with farmers, we realized that there are significant differences in decision making and actions of small, medium and large farmers due to the size of their lands and their economic situation. For instance, large farmers ( $>80$ ha) can buy-out the small and medium farms that have little access to irrigation water, or set up a water desalination system which is a very expensive option for providing good quality irrigation water, or purchase surplus water from small and medium farmers who are no longer harvesting their orchards. Whereas medium farmers (15 to 80 ha), tend to integrate their farms and irrigation systems amongst themselves to increase the efficiency of their lands' irrigation water use and productivity, or modify their irrigation systems from flood irrigation into drip irrigation, something that most large farmers have already done. Small farmers ( $<15$ ha) have fewer options to adapt to water scarcity: these are basically changing the irrigation system or turning off their well pumps during the night or over the winter. There are also 
some common adaptive actions among all groups of farmers e.g. deepening wells or shrinking the orchard size. The extent of shrinking differs based on the location and size of the farms. Because of such differences in behavior, we aggregated the individual maps in three groups of large, medium and small farmers (figure 2 and appendix 8$)^{1}$.

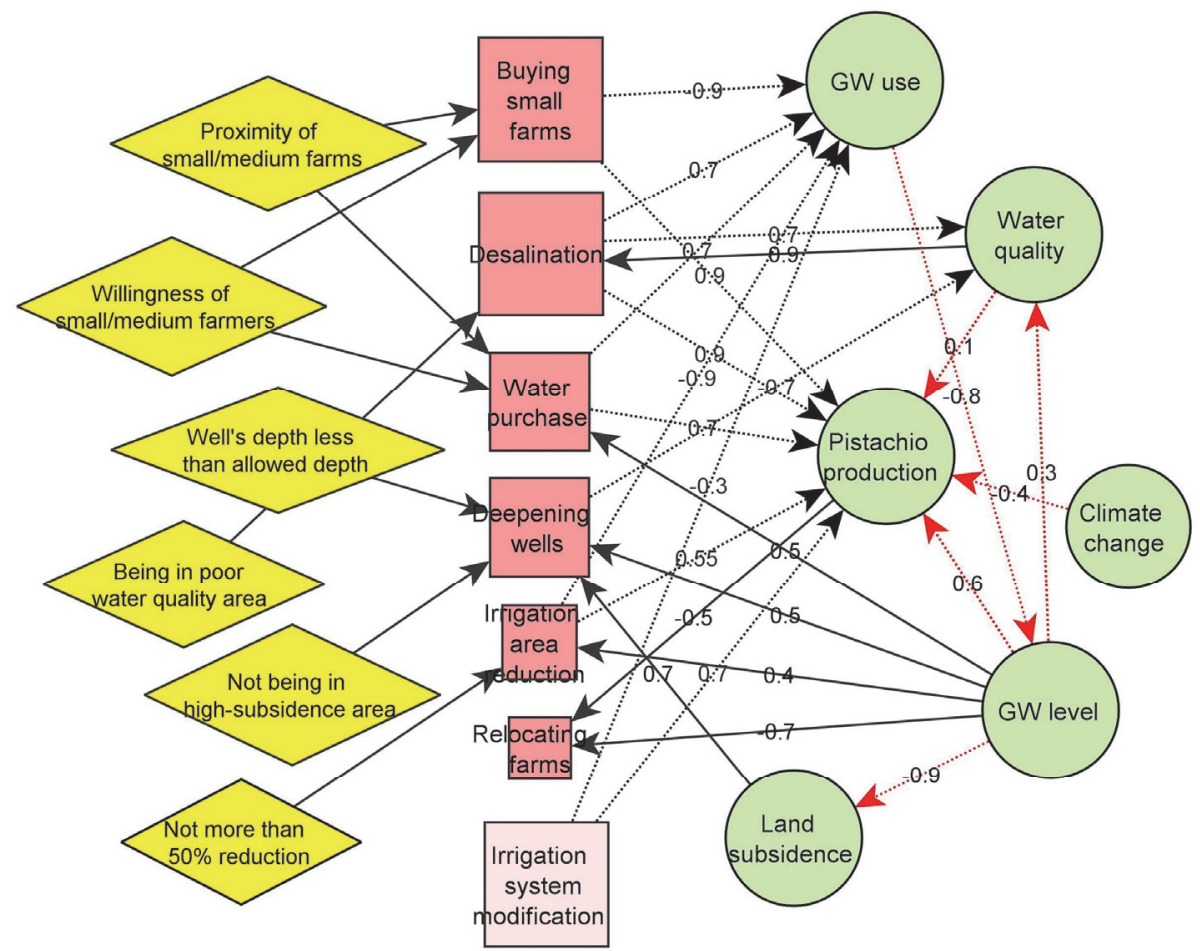

Figure 5.2: Large-farmers' FCM combined with objective data. The red squares show farmers' actions and their size shows the number of farmers who took this action i.e. level of preference or priority of actions. Yellow diamonds are conditions and green circles are either impacts or condition for some concepts and impacts for other. Dashed and solid lines represent impact and driving connections, respectively. Black and red lines represent perceived connections and data-driven connections, respectively. FCMs of medium and small farmers are given in the appendix 8 .

\section{Combining subjective and objective data in FCM}

In modeling SESs, many social and ecological variables interact with each other. For some of these variables we may lack accurate objective data,

1. The initial FCM model that we developed in the fieldwork included a much larger number of variables indicating causes and impacts of water scarcity than what we used in this study. Since the aim of this study was to investigate the impact of farmers' actions on groundwater use and emigration, we only kept the variables relevant to these concepts. However, considering the objective of policy makers and researchers, the size of FCMs can be larger or smaller, by using different simplification methods in FCM (Hatwagner et al., 2018, Lavin and Giabbanelli, 2017) 
however in such cases, we can also rely on stakeholders' knowledge and perceptions due to their close relationship with the environment, e.g. individual land productivity and farmers' vulnerability. For some other variables, we may have access to objective data measured by formal scientific method, e.g. precipitation and groundwater levels. Therefore, both subjective and objective data are crucial and complementary to enable a full understanding of the system changes (Gosselin et al., 2018), particularly for building an ABM. In this step we combined both subjective knowledge derived from stakeholders and the objective knowledge derived from formal scientific studies. First, among all available connections between variables in farmers' FCMs, we identified the connections that (i) are recognized as causal connection by farmers, and (Chapin III et al.) can be measured more accurately with available empirical data e.g. hydrological and ecological variables. Then, such connections received a data-driven value based on correlation coefficients between two variables' time-series data. Since the correlation coefficient alone does not imply causation, we only applied the correlation values to the connections for which the causality has already been determined by farmers. The results of this step are group specific FCMs containing two groups of connections: 1 ) the ones that are perceived by stakeholders (black connections in figure 1, step 1.3), and 2) the ones for which the causality is perceived by farmers and the correlation values are driven from time-series data (red connections in figure 1 , step 1.3). Therefore, such group specific FCMs are combinations of stakeholders' perceptions and data-driven knowledge covering different aspects of an SES.

The list of data-driven connection values developed by available time-series data and validated by stakeholders' perceived FCM is summarized in appendix 10. These data-driven values were therefore used instead of perceived-values in all three group-specific FCMs, to cover the ecological and data-abundant part of the system (red connections in figure 2). Yet all other connections, including those representing the causes and impacts of actions, remained with their perceived values obtained from farmers (black connections in figure 2).

\subsubsection{Step 2: Translating FCM to ABM ODD protocol}

We used the ODD protocol for describing the ABM (Grimm et al., 2010). The ODD protocol is a standard framework of elements that need to be covered when developing and describing an ABM. It requires descriptions of entities in the model, their characterized attributes and behavioral rules (which entity does what, in what order, what rules do entities have for making decisions or changing their behavior in response to environmental changes), and model rules (what are the direct interactions among entities and indirect interactions via environmental variables) (Grimm et al., 2017). Many of these elements were extracted from FCM models developed in step 1. 
A full ODD description is given in appendix 8. Below, we provide a summary of the ODD.

Agents represent a total of 154 farmers in three groups: 21 large-farmers, 49 medium-farmers, and 84 small-farmers (section 3.1.2). These farmers are distributed across a stylized representation of the Rafsanjan landscape, distinguished by nine sub-regions in the ABM, out of which two represent nonvegetated areas (i.e. arid land). Each sub-region consists of 15 by 15 cells, leading to a total of $45 * 45$ cells (figure 3 , details on initialization based on empirical data are given in appendix 8 ). Each cell can be owned by one farmer; each farmer may own 1 or more cells. Agents are distributed equally in the seven regions and randomly within each region (figure 3 ). Each cell represents 5ha of pistachio land. Cells are characterized by: 1) Depth of groundwater level, 2) Groundwater quality, 3) Land subsidence level, 4) Groundwater use 5) Well depth, and 6) Allowed well depth.

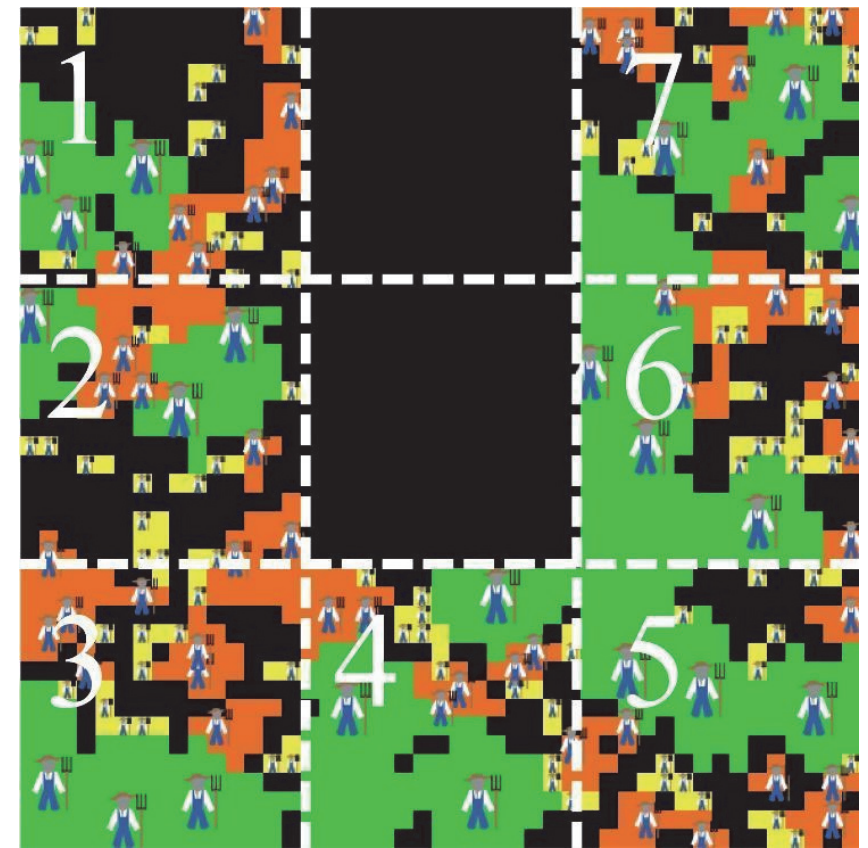

Figure 5.3: Set-up and allocation of farmers and farms in Netlogo. Green, orange and yellow cells represent large, medium and small farms, respectively. The two black regions in the middle are not farming regions (to represent the real U-shape landscape of Rafsanjan).

Temporal resolution: The time step is 1 month, and variables' changes are monthly or yearly. The time horizon of the model is 15 years, i.e. 180 time steps. 
Process overview: Within each time step two main activities take place in the following order:

1) Cells' update: There are two types of updates for each cells' properties: 1 ) based on variables' dynamic changes collected from empirical data, e.g. groundwater level change and land subsidence level change, 2) based on impacts of actions from the previous step on environment variables.

2) Agents' decision-making: First, each agent checks its groundwater access. If the agent is not satisfied with the groundwater access, it enters a decision making process to adapt its groundwater access. Otherwise, it exits this time step.

Agents' decision-making: At each time step, agents observe the environmental situation of their cells and make a decision. Therefore, all agents have full knowledge about the state of their groundwater access, groundwater quality, land subsidence, their neighbors' willingness to sell their water/lands, and the execution of different policies. The possible actions that each group of agents can take are listed in table 1 . Their decision-making is described using CAI diagrams (section 3.3.2) and formalized in UML activity diagrams (section 3.3.3). 
Table 5.1: The set of possible actions that can be taken by large, medium and small farmers.

\begin{tabular}{lll}
\hline Action & Description & $\begin{array}{l}\text { Farmers who take } \\
\text { this action }\end{array}$ \\
\hline $\begin{array}{l}\text { Buying } \\
\text { small/medium } \\
\text { farms }\end{array}$ & $\begin{array}{l}\text { Buying farms from medium or small } \\
\text { farmers who are not willing to } \\
\text { continue pistachio production }\end{array}$ & Large farmers \\
\hline Desalination & $\begin{array}{l}\text { Set up desalination system on farms } \\
\text { with saline groundwater to remove } \\
\text { salt and minerals }\end{array}$ & Large farmers \\
\hline Water purchase & $\begin{array}{l}\text { Buying water from medium or small } \\
\text { farmers who are not using their } \\
\text { well's water for irrigation }\end{array}$ & Large farmers \\
\hline Deepening wells & $\begin{array}{l}\text { Digging water wells to get access to } \\
\text { groundwater }\end{array}$ & Large/Medium farmers \\
\hline Irrigation area & $\begin{array}{l}\text { Shrinking (dry-off) small part of the } \\
\text { farm to increase the efficiency of } \\
\text { water use for rest of the farm }\end{array}$ & Large/Medium/Small \\
\hline Integrating farms & $\begin{array}{l}\text { Integrate irrigation systems of } \\
\text { several farms to increase their } \\
\text { efficiency }\end{array}$ & Medium farmers \\
\hline $\begin{array}{l}\text { Irrigation system } \\
\text { modification }\end{array}$ & $\begin{array}{l}\text { Changing traditional flood irrigation } \\
\text { to drip irrigation }\end{array}$ & Medium/Small farmers \\
\hline Well's turn-off & $\begin{array}{l}\text { Increasing the wells' off-time } \\
\text { (overnight or during winter) }\end{array}$ & Small/farmers \\
\hline Relocating farms & $\begin{array}{l}\text { Leave the region and buy a farm in } \\
\text { another area with a better water } \\
\text { situation }\end{array}$ & Large farmers \\
\hline
\end{tabular}

\section{CAI diagrams}

At an abstract level, the behavior rules in an ABM constitute the set of actions that agents might take, the conditions under which these activities take place, and actions' outcomes (impacts). The set of actions and order of actions stemming from the FCMs can be used in constructing the behavioral rules, and conditions and impacts of actions can be defined by inputs and outputs of those actions in FCM. Therefore, a set of Conditions-Action-Impacts (CAI) for each group-specific FCM is produced in this step, covering three main concepts of decision-making:

- Set of actions: represent different actions taken by each group of stakeholders. The priority of actions is represented by the number of times they have been mentioned by stakeholders as their chosen adaptive action (shown by the size of action nodes in FCM, figure 2). Therefore, higher priority actions have a higher preference for farmers/agents to be implemented. However, the preference order may not be the actual order of decisions taken by stakeholders, since some actions cannot be performed in some locations or during some months of the year). These two aspects are added later in the ABM implementation. 
- Conditions of actions: are input concepts of each action representing driving forces or causes of that action. Condition actions can be either dynamic e.g. groundwater level in figure 2 (accompanied with weighted connections to actions), or fixed (true/false) variables, e.g. proximity of farm in figure 2 (accompanied with connections without weight).

- Impact concepts: are output concepts of each action along with their causal network, i.e. direct and indirect impacts of that action. Impact concepts are dynamic variables (with changing states) ${ }^{2}$.

Figure 4 indicates the series of CAI diagram transferred from large farmers FCM. The CAI diagrams for medium and small farmers are shown in appendix 8. For example, for the first action of large farmers i.e. buying small/medium farms the conditions are proximity of small/medium farms to the large farm and willingness of their owners to sell-off their farms. Thus, this action is possible for large farmers when there is at least one small or medium farm in their proximity whose owner is no longer willing to harvest pistachio and who is also willing to sell the land. This action affects pistachio production and groundwater use with different levels of influence, based upon the largefarmers' FCM. Likewise, these two variables affect groundwater level, groundwater quality, pistachio production and land subsidence, which are the indirect impacts of action 1. Moreover, actions are prioritized based in their node size for each group separately, and the nodes with the same or similar node size have the same priority.

\footnotetext{
2 One concept in FCM can be a condition for some actions and impact for others. The function of each concept is defined in relation to its connection (input or output) with action concepts (figure 1, steps 1.1 and 1.3).
} 


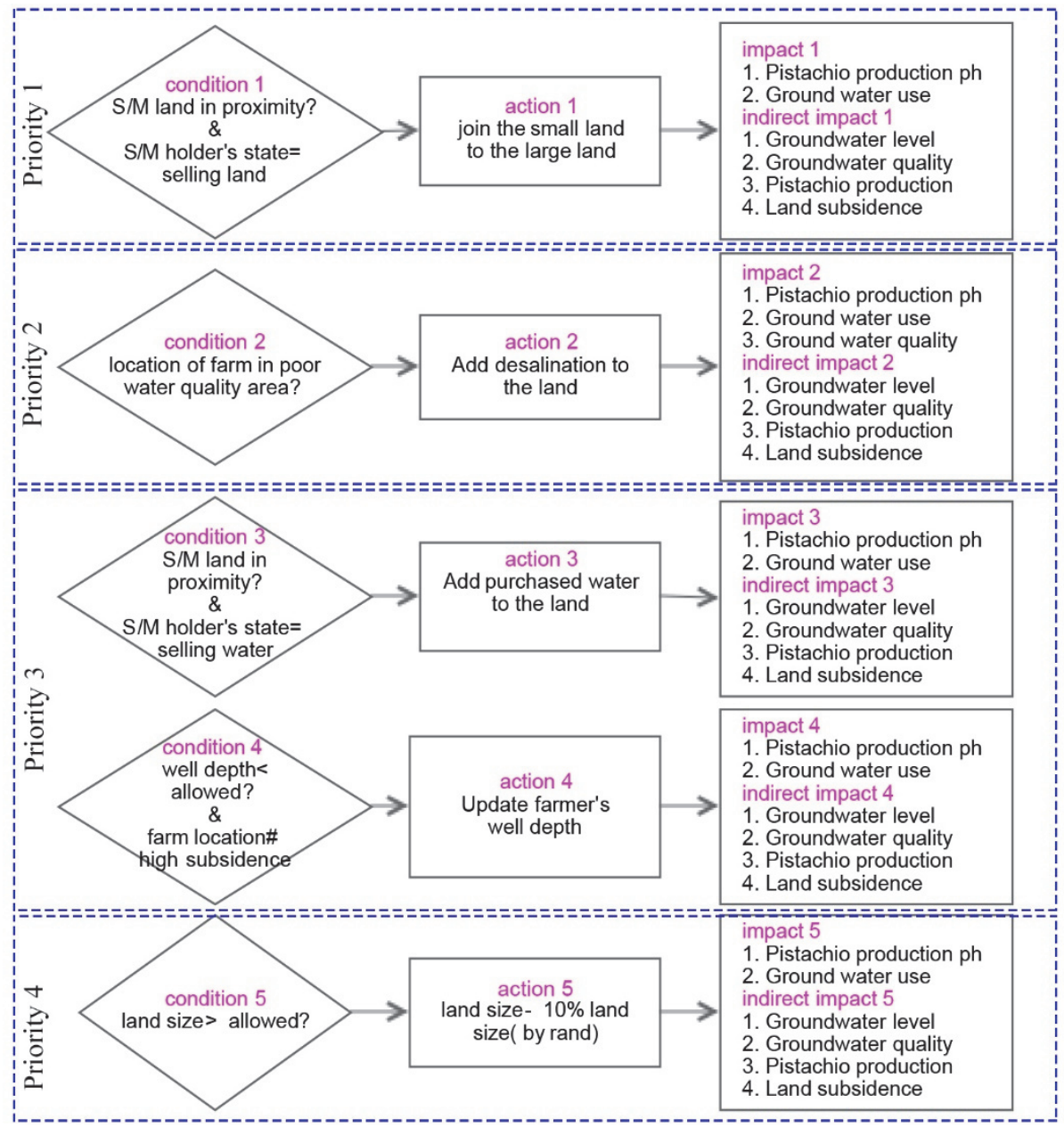

Figure 5.4: CAI of large farmers that represents set of conditions and impacts for each specific action. S/M: Small/Medium, ph: per hectare.

To implement the direct impact of actions $X$ onto variables $A$ of the FCM model (represented as $X \stackrel{w}{\rightarrow} A$ ), in each time step that action $X$ has executed the value of Variable $A$ in that time step is calculated as:

$$
A_{t+1}=A_{t}+\left(A_{t} \times w\right)
$$

For example, when we have desalination $\stackrel{0.7}{\rightarrow}$ groundwater use (in figure 2), whenever that action desalination is executed, it impacts groundwater use by 0.7 of its current value. So Groundwater use $\mathrm{t}_{\mathrm{t} 1}=$ Groundwater use $\mathrm{t}+$ (Groundwater use $\mathrm{t} * 0.7$ ). 
All indirect impacts of actions are calculated at the beginning of the next step (in the cell's update step in section 3.3.1). Indirect impacts of actions are the impacts of variables affected by actions on other variables in FCM. To implement the impact of Variable $A$ onto the Variable $B$ (represented as $A \stackrel{w}{\rightarrow} B$ ) the value of Variable $B$ in the new time step is calculated as:

$$
B_{t+1}=B_{t}+B_{t} \times \frac{A_{t}-A_{t-1}}{A_{t-1}} \times w
$$

The direct and indirect impact of actions may also take the role of condition for the same or other actions in the next time step, which represent feedback loops in FCM (e.g. loop of water purchase $\rightarrow$ groundwater use $\rightarrow$ groundwater level $\rightarrow$ water purchase, in figure 2 ).

\section{Building UML diagram}

Unified Modeling Language (UML) was used to develop the ABM structure. UML proposes a set of well-defined and standardized diagrams to design and describe a system before coding it (Bersini, 2012). One of the most commonly used UML diagrams with ABM is the activity diagram, which represents the sequential steps of actions and timing of processes (Bersini, 2012, Elsawah et al., 2015). To transfer CAI diagrams into UML diagrams, there are some crucial aspects that cannot be collected and represented in FCM i.e., randomness, temporal and spatial dimensions. We added such aspects by using quantitative data from studies and government reports, and estimates based upon local knowledge collected during interviews. Such aspects have been added to each actions' priorities and conditions and initial values of parameters.

- Time scale: Actions may be taken by farmers every month, every six months or every year. Moreover, some actions can be taken by farmers only once (e.g. desalination or irrigation system change), whereas some other actions can be taken several times until their limits are reached (e.g. well deepening or land shrinking). Therefore, the time scale (i.e. frequency and one-time or repetitive) are added to the condition of each action. Thus, if an action is executed annually, the condition for this action is to be in time step multiples of 12 .

- Randomness: Randomness is added to the priority set of actions in the behavioral rules of agents as well as in the initialization of parameters' values. In the priority set of actions, some actions have the same or very similar priority ${ }^{3}$. In these cases, one action is randomly chosen to have

\footnotetext{
${ }^{3}$ When the number of times two actions mentioned as preferred action by stakeholders differs by less than 3 , i.e. 0.05 of the total population, we consider them as similar priority actions.
} 
priority over the other. Randomness is also used in the distribution of agents over the seven sub-regions, as well as their farm sizes within the ranges of small, medium and large farms' area mentioned in section 3.2.2. For the initialization of parameters' values, an interval of initial values was collected for each parameter in each sub-region and randomly distributed over the farm patches (appendix A, section 3.1).

- Spatial dimension: Some environmental properties have significantly different values in different regions of Rafsanjan. For example, groundwater quality and land subsidence level are different in each of the seven sub-regions and thus have a different impact on farmers' decisions. This spatial heterogeneity is represented in the cells' properties and added to the conditions of each action.

In appendix 8, the UML activity diagram of large farmers (i.e. the sequence diagram of farmers' decisions and actions) is shown as an example. This UML diagram shows that at each step, agents first check their actions' conditions through their priority order of actions. If the conditions are confirmed they execute the action, giving rise to associated impacts. If the conditions are not met, they go to the next action. If a small or medium farmer reaches the end of the action list the final action is to sell the farm to a large-farmer and leave the region. For large farmers, their final action is to relocate their farms to outside the region.

\subsubsection{Step 3: ABM implementation and assessment}

In this step, the ODD and UML activity diagram from the previous section was used to build the pseudo-code and then translate it into an actual code implementation. We used the Netlogo 6.0.1 platform to implement the ABM (Wilensky, 1999). The source code of this model can be found online in "CoMSES Computational Model Library" (www.comses.net).

As calibration of the model, we followed the stepwise-design approach suggested by Sun et al. (2016) i.e. starting with a simple model version that captures basic processes and then, adding more detailed processes and components to the model structure such that the relative importance of each component could be quantified and assessed along the way. For example, we started first with the same initial well's depth and groundwater level for all cells of each region. This resulted to a staircase-like groundwater use for each region since all agents would lose groundwater access and start taking action at the same time. Therefore, we added variety of wells' depth and groundwater level in different cells to model the heterogeneous reactions of farmers at each time step. By adding more details, a point was reached eventually at which model performance failed to improve, either due to the irrelevance of those additions to the objective of the model (i.e. details that had no impact on 
groundwater use or farmers migration in our case) or over-complexity of model (e.g. level of complexity suitable for quantitative prediction and scenario analysis rather than comparing impacts of different policies such as our case). That is where we stopped adding more details to the model-other approaches are proposed in Edmonds and Moss (2004) and Sun et al. (2016).

\section{Validation}

Historical data on groundwater use for 2004 to 2012 were used to validate the simulation model. The idea was to see how well this model structure replicates the historical reality. To align with reality, the validation model only simulates the implementation of actions that were available in the past, but with the same level of impact, conditions, etc. as the present. First, the four environmental parameters (groundwater level, well's depth, groundwater quality, and land subsidence) were initialized with their values in the year 2003. Second, desalination, water marketing, and land integration were removed from the validation model, since such actions are recent adaptation actions taken by farmers. Moreover, irrigation system change was still an option for large farmers over the period 2004-2012, so this action is added to the action set of large farmers for the validation. The values of both simulation and reality data-sets were normalized to show the percentage of changes.

\section{Baseline scenario and policy options}

First, the baseline scenario was simulated. In this, agents decide and act based on their current situation and without any policy interference. Besides simulating the current situation, we also need a set of simulations to compare the impact of different policies that influence farmers' decisions and actions. Among current government policies toward water scarcity (Kerman Provincial Government, 2014, Mehryar et al., 2015) , we chose three that aim to reduce groundwater use by changing behaviour and actions of farmers:

Policy of shrinking lands: This policy focuses on decreasing the irrigation water use by reducing the areas used for pistachio production. To implement this policy, the government buys-off parts of the farms and changes their land use to non-agriculture activities. Based on our field work experience and due to the severity of water scarcity in Rafsanjan, many farmers agree to sell-off some of their lands, but only to an extent that still enables them to profit from production.

We implemented this policy by removing actions of land marketing and water marketing between large and small farmers, since as a result of this policy, small and medium farmers sell their lands to the government instead of large farmers. 
Policy of irrigation system change: This policy focuses on replacing current flood irrigation systems with a drip irrigation system. To encourage farmers, the government provides an irrigation modification subsidy for farmers with land tenure documents. Currently, about $50 \%$ of the small farmers and $30 \%$ of the medium farmers do not have land documents due to the informal exchange of lands during the 1978 revolution. Therefore, the lack of land documents is the main obstacle for farmers who cannot afford to independently finance expensive drip irrigation systems. In this policy, the government aims to remove the land document problem and provide a subsidy to all farmers.

We implemented this policy by removing the condition of land documents for small and medium farmers. Therefore, all medium and small farmers who reach this action in their priority list execute irrigation system change.

Policy of farmer participation: This policy focuses on encouraging and involving farmers to reduce their water use by decreasing the priority of actions that increase their groundwater use like desalination and well deepening, as well as increasing the priority of actions that reduce their water use like integrated farming.

Implementation of this policy was done by removing desalination, water purchase and well-deepening, and adding farm integration to large farmers.

The setup of the simulation experiments is as follows. The validation cover the period from 2004 to 2012, thus 96 time steps. New policies were explored for the time period of 2015 to 2030 (i.e., 180-time steps), and the environmental parameters were initialized with their values in 2015. For each combination of parameters, 100 simulation runs were analyzed. Confidence intervals for the acquired mean values of major output variables suggest that this amount of simulation runs led to satisfactorily precision (Figure 5, Figure 7).

\section{Sensitivity analysis}

We applied one-factor-at-a-time (OFAT) sensitivity analysis to explore the relationships between the model output and input parameters. OFAT consists of varying one parameter at each time over a wide range of its possible values while keeping all other variables fixed (Ten Broeke et al., 2016) and thereby, monitoring changes of the simulation model output. OFAT helps to identify those parameters that have a strong influence on model output, and are therefore most important (Thiele et al., 2014). However, OFAT does not take into account the simultaneous variation of input variables, thus does not detect the presence of interactions between input variables. To show the form of relationship between the interacting variables and the output other methods such as Regression-based analysis, and Sobol model (Ten Broeke et al., 2016) can be used. 
We used OFAT to evaluate the influence of: 1) parameters' changes on groundwater use including impact values derived from FCM model and thresholds derived from hard data and estimated data, 2) stochasticity in our model results (i.e. random processes used in the initial distribution of farm sizes, initial well depths and choosing between actions with the same priority). A full list of parameters with their range of values used for sensitivity analysis is shown in appendix 9.

\subsection{Results}

\subsubsection{Baseline scenario}

The result of the baseline scenario (i.e. the impact of aggregated farmer's decisions and actions on overall groundwater use), is shown in figure 5 . Due to a lack of space, we do not report on actions taken by individual farmers. We explain these results in pairs of regions that show similar results.

Regions 4 and 5: Farmers in these two regions still can deepen their wells at the beginning of the simulation, while other regions have either very poor water quality or very high land subsidence that prohibit more well deepening (appendix 8). Well deepening and water marketing in regions 4 and 5 results in a rapid rise in their aggregated groundwater use. The peaks of groundwater use in these two regions occur when farmers reach their permitted well depth, at which time further deepening stops. Hereafter, trends of groundwater use are followed by a slight decrease due to actions like shrinking lands and buying/integrating farms. Since region 5 has better access to groundwater than region 4 (appendix 8), farmers in region 5 start taking adaptive actions later than those in region 4 . Therefore, the groundwater use in region 5 lags slightly behind that of region 4 .

Regions 1 and 2: These two regions have very poor water quality in the lower layer of their aquifer, thus deepening wells is not a useful option for their farmers. Facing low water access, large farmers install a desalination system which has a very high, though short duration, impact in increasing their groundwater use. Thus, after a short term peak in groundwater use, region 1 shows a steady decrease of groundwater use due to buying/integrating farms, land shrinking and irrigation system change. In region 2, after the initial peak, there is another slight increase in groundwater use because of water marketing between small and large farmers which is feasible in the southern part of this region.

Regions 3 and 6: Parts of regions 3 and 6 do not allow for more well deepening due to poor water quality and land subsidence, respectively. Farmers in both regions start with buying/integrating land and irrigation system change at the 
beginning (when the water scarcity is less). With these two actions, they reduce their water use and increase their water access, both at a relatively low level. After about 5-6 years, farmers who can deepen their wells and purchase water, which increases groundwater use. After meeting their allowed well depth and the buy-out and emigration of small/medium farmers, they continue mostly by shrinking lands in order to steadily reduce their groundwater use.

Region 7 has the best water situation, in terms of both access and quality, but faces high land-subsidence which prohibits more well deepening. When farmers face water scarcity, their available actions are buying/integrating lands, shrinking lands and irrigation system change, all of which reduce groundwater use to some extent. Therefore, region 7 shows a constant decrease of groundwater use.

Overall, all regions face a slight and constant decline of groundwater use after meeting their peaks-either at the beginning or in the middle of simulation process, at which time the farmers have no other options than shrinking farms or selling their farms to the farmers who still have access to groundwater. This only happens after farmers meet limitations of other actions e.g. well deepening and well termination and/or accomplish all one time actions e.g. desalination, irrigation change and farms' integration. Therefore, such groundwater use reduction only happens after a large increase of groundwater consumption by farmers which is followed by emigration of farmers.

\subsubsection{Validation}

To compare the results of groundwater use in simulation and reality we used a two-sample Kolmogorov-Smirnov test. The result shows that we do not have enough evidence to conclude that there is a significant difference between the simulated and the real data-D-stat $(0.0122)<D$-crit $(0.0123)$. There are two specific peaks of groundwater use, both in the simulation and in the real data (Figure 5). Such peaks, in reality, are because of significant well deepening in different regions (i.e. first in sub-regions 1 and 2 and later in sub-regions 6 and 7), where currently most of the wells have already reached their maximum depth. The difference between the two charts after 2011 (Figure 5B) is because of the introduction of new actions by farmers i.e. desalination and water marketing. Therefore, their impacts are not seen in the simulation runs for validation where these two actions were removed. 


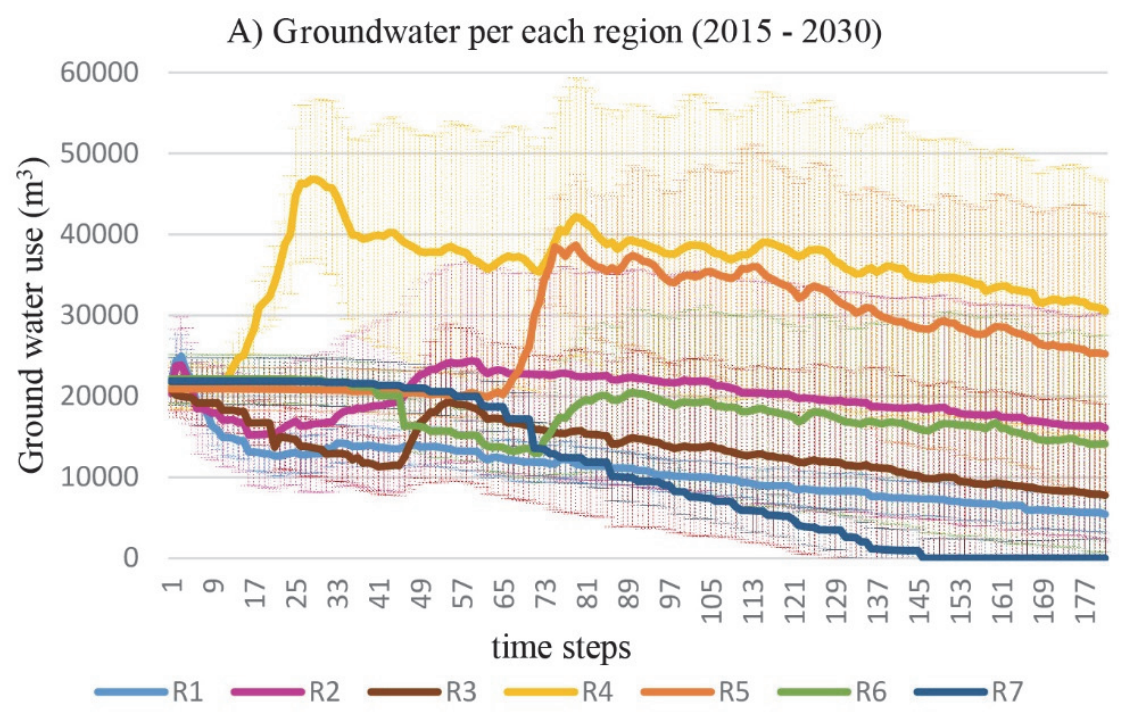

B) Overall groundwater use, 2004-2012

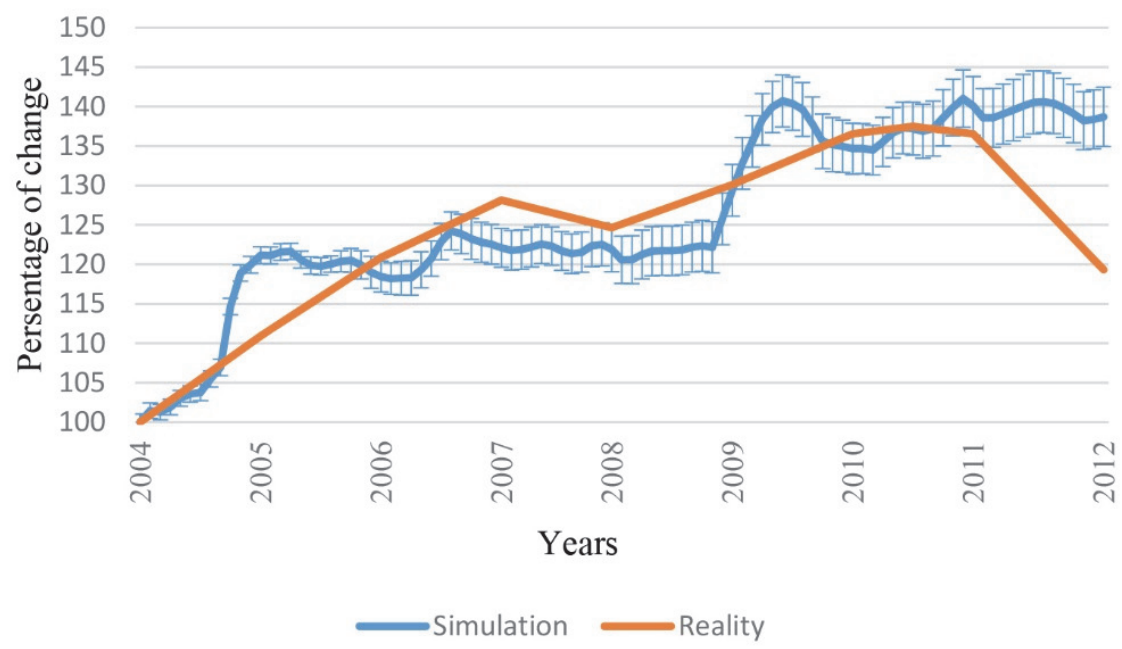

Figure 5.5: A) Groundwater use per region (for all groups of farmers) in the baseline scenario (2015 - 2030). The shaded areas depict standard deviation for each region over 100 time simulations. R: region. B) Validation using groundwater use of whole Rafsanjan in simulation and reality over the period 2004-2012. Due to difference in initial values of simulation and reality, their data-sets are normalized to show the percentage of changes. The bars depict confidence intervals over 100 time simulations. 


\subsubsection{Policy options simulations}

Simulating the impact of different policy options revealed striking impacts on groundwater use overall and in the different regions (Figure 6):

The policy of shrinking lands has a strong impact on reducing groundwater use because it also implies that water and land marketing are no longer feasible in the region. Yet, it results in higher emigration of farmers than in the other policy scenarios (Figure 7).

The policy of irrigation system change is very similar to the baseline scenario. This is due to the past experience of irrigation system change among large farmers. According to large farmers' perceptions (Figure 2), changing the irrigation system to drip irrigation has not changed their water consumption, but has been used by farmers to expand their pistachio area and/or increase the productivity of their lands. Therefore, this policy has a positive impact in encouraging medium-farmers and small-farmers to stay in the region, since it helps to improve their production quantity and quality.

The participation policy has the highest impact on reducing groundwater use and keeping farmers in the region. Stopping the high water consumption actions e.g. well deepening and desalination, besides focusing on reducing water demand by farm integration and reducing farm areas shows the largest reduction on overall groundwater use compared with other scenarios. Moreover, it has the least impact on emigration of large farmers and after the irrigation change the least impact of emigration of medium and small farmers.

The results of baseline and irrigation change scenarios in regions 2-6 have a large standard deviation range (Figure 6). The sensitivity analysis of all parameters for such policies indicates well deepening as the most sensitive parameter. Regions 1 and 7 are the only regions that do not have the action of well deepening, and thus simulation of all policies in these two regions shows a small standard deviation range. Similarly, policy options of land shrinking and farmer participation are the only scenarios that do not change the execution or impact of well deepening, thus they also show a small standard deviation range in all regions (orange and yellow lines in figure 6). 

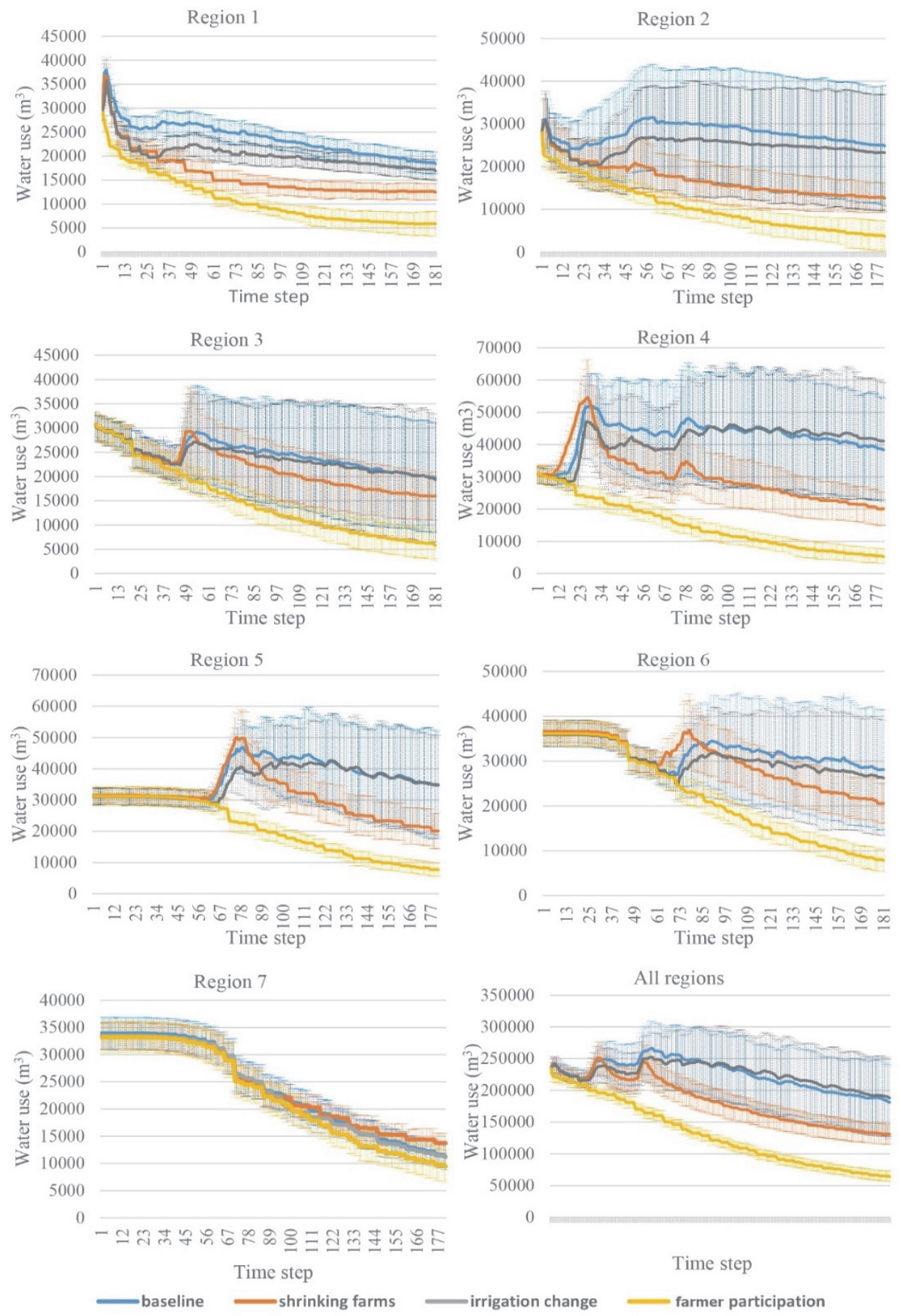

Figure 5.6: Groundwater use per region and overall groundwater use in three policy options scenarios compared to the baseline. The shaded areas depict standard deviation for each scenario over 100 time simulations. 


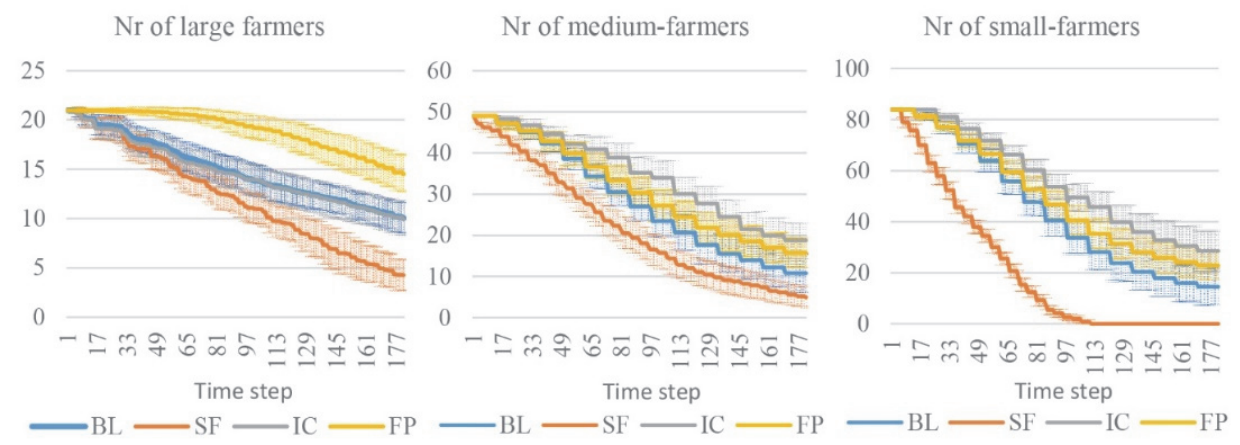

Figure 5.7: Number of large, medium and small farmers as a function of time in three policy scenarios compared to baseline. BL: baseline, SF: shrinking farms, IC: irrigation change, FP: farmer participation. The shaded areas depict standard deviation for each scenario over 100 time simulations.

\subsubsection{Sensitivity analysis}

The results of the sensitivity analysis (shown in appendix 9) indicate that well deepening and land shrinking on groundwater use have the largest influence on the overall groundwater use in Rafsanjan. By contrast, desalination has the least impact on groundwater use, though it has a high impact value in the FCM. This is because very few farmers actually execute this action either because of their farms' location (i.e. being in good groundwater quality regions), or because of their economic situation (i.e. not being able to afford to install and operate desalination systems).

Sensitivity analysis of random processes shows that changes in the spatial distribution of farm cells during initialization and initial values of well depths per cell do not lead to distinctly different outcomes, meaning that the model is not sensitive to these two random processes. However, the results show high sensitivity to the random choice between actions 3 and 4 of large farmers (i.e. water purchasing and well deepening). Specific, if the model always executes action 3, water purchasing, the results show little sensitivity, whereas, if the model executes either always action 4, well deepening, or a random choice between these two, the results show high sensitivity. This highlights again the important role of the well deepening action on the overall groundwater use.

\subsection{Discussion}

To support effective policy making in SESs, a policy simulation has to consider the multi-factorial behavior of the system as well as multi-stakeholders' decision-making and the impact of these decisions on the physical system. Consequently, integrated methodological approaches for policy simulation in SESs are required. This paper shows how a combination of FCM and ABM methods for simulating impacts of policy options in SESs could be useful. In 
this section, we reflect on our methodology by presenting its strengths, limitations and suggesting possible future improvements.

\subsubsection{Strengths}

Our study showed that FCM and ABM are complementary and together can cover the four main features of an SES for policy making purposes: 1) Causal relationships between human actions and their surrounding social and ecological factors. FCM represents the decision making process of stakeholders and their impact on the environment in a causal directed graph. Therefore, it shows how each action causes direct and indirect changes in environmental variables. 2) Feedback mechanism: FCM's outcomes explicitly incorporate feedback in human-environment interactions (e.g., the positive and negative impact of an action on environment reinforce a subsequent action). 3) Socialspatial heterogeneity: ABM incorporates various stakeholders' preferences, available actions and long-term goals (i.e. individual heterogeneity) and it involves various environmental properties in different locations (i.e. spatial heterogeneity). 4) Temporal dynamics: ABM can represent time scale in agents' actions and environment variables, (e.g. slowly changing variables such as population change) vs. fast-changing variables (e.g. annual agriculture production) or high-frequency actions (e.g. farm irrigation) and low-frequency actions (e.g. buying lands).

In addition, the combined use of FCM and ABM in a modelling process is useful to formulate and parametrize the qualitative knowledge gained by stakeholders, combine it with quantitative knowledge from "hard" data and use both data types in simulating human-environment interactions. Our proposed modelling framework is particularly useful for policymakers to incorporate human perceptions, preferences, decisions and actions in the process of exante policy options analysis. Moreover, it provides the macro level observation of the system's elements, (i.e. multi-variables interactions), as well as the micro level view of the individual interventions and decision-making, which supports comprehensive policy analysis.

\subsubsection{Limitations and future studies}

One limitation of the FCM method is its limitation in defining the nonlinear relationships between concepts (Voinov et al., 2018). For example, using FCM gave us the immediate and fixed impact of actions on variables, which resulted in presenting the linear relations among variables. However, some actions' impacts may be nonlinear (i.e., adapt dynamically and increase or decrease over time). In this study, we used the traditional FCM method since the focus of our study was on translating FCM causal relationships and feedback loops into behavioural rules of ABM. However, there are some extensions to the FCM methodology to capture nonlinearities. Rule-Based Fuzzy Cognitive Map 
(RBFCM) (Mourhir and Papageorgiou, 2017, Carvalho and Tomè, 2000) is an approach that captures and represents relations other than monotonic causality between concepts, thus can better show the dynamic impact of actions on variables. Replacing FCM with RBFCM in this method is proposed for future studies involving the dynamic impact of actions.

Second, an aggregated FCM represents the average of all individual FCMs. In our study, the variability of stakeholders' preferences, decisions and actions are represented by grouping FCM models for large, medium and small farmers. In some applications, it is necessary to take into account the distribution of stakeholders' perceptions even within each group. Therefore, another interesting approach or extension to this work would be to use interval (or standard deviation) instead of a fixed average value for the FCM connections' weights and apply randomness within the range of values in each time step. In this way, the variation of collected data from stakeholders can be used in describing the impact of agents' actions in ABM. However, larger sample sizes for each group would be beneficial when doing so.

Third, in this methodology, the ABM simulations cannot predict the accurate value of social and ecological variables, since the FCM connection weights are fuzzy and comparative values by stakeholders' perception. Therefore, they are proper for comparing the impact of different policy options but not predicting the future of the system or accurate impacts of each scenario on variables' changes.

Fourth, learning and prediction are two important properties of many ABMs. In this study, we did not integrate these two aspects as agents' properties. However, for future studies, farmers' abilities to learn from their experiences, adapt their actions and estimate future consequences of their decisions could also be added to the simulation model.

Fifth, validation of the model has been done for the whole region due to the availability of historical groundwater use data only for the whole region but not for each specific sub-regions. However, in the case of data availability, validation of simulation for each sub-region separately would provide more confidence in the model.

Last, ODD+D protocol (Müller et al., 2013) can also be used in this methodology instead of standard ODD. This protocol rearranges the design concepts to better capture human decision-making. 


\subsection{Conclusion}

This study introduces a step-wise methodology to integrate a factor-based modelling approach (i.e. FCM), with an actor-based modelling approach (i.e. ABM), to support policy option analysis in SESs. In this methodology: 1) FCM aggregates the qualitative stakeholders' knowledge and perception to model the SES function and stakeholders' adaptive reactions to the system, 2) the output of FCM is translated to be used as ABM input data 3) ABM is developed to simulate and compare the impacts of different policy alternatives considering human-environment dynamic interactions. We applied this methodology for the case of a farming community facing water scarcity in Rafsanjan, Iran. The results show that this integrated methodology takes into account aspects of complex SESs that cannot be fully covered by either modelling approach if used individually.

Moreover, our case study indicates that among three policies of shrinking farms, irrigation change and farmers' participation, the policy of shrinking farms is a high incentive policy for farmers to reduce their irrigation areas and thus decrease pressures on aquifer and groundwater use. However, due to the high emigration of farmers in this scenario, it is not a satisfactory policy from a socio-economic perspective. Rather a policy to facilitate farmers' participation in the management and control of their groundwater use has the highest impact in reducing overall groundwater use, and it reduces emigration. Surprisingly, adapting new irrigation technologies does not have any significant impact on reducing overall groundwater use in the region. 
Chapter 6

Synthesis 


\subsection{Conclusions}

The aim of this research was to develop and evaluate methods to enhance public participation (e.g. resource users, policy makers, managers) in policy making for a Social-Ecological System (SES). Implementation and evaluation of such methods have been demonstrated on the case of policy making for a farming community facing water scarcity in Rafsanjan, Iran. An overview of the findings for each of the four objectives is given below.

\subsubsection{Objective 1: Developing an analytical framework to understand the social-ecological drivers and impacts of water scarcity in Rafsanjan, Iran.}

Chapter 2 provides a holistic overview of water scarcity in Rafsanjan and its social-ecological drivers and impacts. A social-ecological analysis of the case, represented in a Drivers-Pressures-State-Impacts-Responses (DPSIR) framework, reveals that rapid and perhaps ill-conceived economic development of Iran, in conjunction with weak groundwater governance, has had the most destructive impact on water resources in Rafsanjan. Iran's development plans for this region have mostly focused on short-term economic development, self-sufficiency and self-reliance, regardless the potential longterm environmental costs. Besides, the historical analysis of water management and governance in Rafsanjan reveals the increasing exclusion of water users from water governance. This has been driven by nationalization of water and later reinforced by inadequate laws and their unequal enforcement. Unlike the collective water management of the traditional Qanat system, the new "water as a common pool" strategy has reduced peoples' participation and their role in water resource management, which has led to the current situation of groundwater over-exploitation.

The current state of groundwater indicates that Rafsanjan has already consumed most of its groundwater supplies. Satellite image analysis in 2015 indicates that around 30 percent of the approximately 110,000 ha of pistachio lands in Rafsanjan have lost their vegetation. Moreover, local farmers' perceptions are that 50 percent of pistachio lands have lost their productivity. Desertification and migration are the current and anticipated future impacts of the water scarcity. Rafsanjan plain is earmarked as a "critical prohibited plain" where stabilization of the aquifer's decline is the main goal. Moreover, local farmers are losing the capability to maintain their pistachio orchards. Massive transfers of pistachio farms to other plains is now occurring, but this will likely export the water crisis to other regions too.

An inter-basin water transfer project is a government policy to respond to water crisis and pistachio production problem. Besides the high costs of water transfer, this action needs careful consideration given the threat of inter-basin 
conflicts, and an expected further expansion of agricultural land in the region. Other water use control policies and actions from the government, like water pricing, well control and irrigation system modification may not be effective if they do not consider effective participation of all stakeholders in water conservation practices. Moreover, a "one size fits all" solution to the water crisis is not possible. The spatial-temporal differences of water quantity and quality issues in Rafsanjan, and their various socio-ecological impacts require new integrated approaches to support decision-making in such SES.

\subsubsection{Objective 2: Integrating stakeholders' knowledge and perception in modelling SESs and evaluating acceptance of policies by stakeholders}

In chapter 3 a mixed-method for involving stakeholders' perception and knowledge in the process of SES modelling and evaluating policies' impacts is presented. A participatory modelling method (i.e. Fuzzy Cognitive Mapping FCM) is combined with a problem structuring method (i.e. DPSIR). FCM is used to collect and present the perceptions of 60 farmers and 40 policy makers over causes and impacts of water scarcity, and DPSIR is used to structure and categorize the complex FCMs for environmental policy analysis. This mixedmethod allows the simulation of the possible impacts of different water scarcity policies through stakeholders' perceptions and knowledge.

Rafsanjan clearly needs two types of strategies to increase its resilience in dealing with water scarcity. First, quick and short-term actions to fix the most urgent problems, like the loss of pistachio orchards, and agricultural investments. Second, longer-term solutions to secure the sustainable groundwater management. The policy option impact analysis of this study indicates that farmers perceive the change of the economy to be the most effective water scarcity policy option, while policy makers and researchers favour increased government control and monitoring. However, the modelling clearly shows that the farmers have a low level of trust in the ability of the government to regulate and control water usage. The current water crisis appears to justify this view. Therefore, a crucial issue for effective water management in Rafsanjan would be the equitable engagement of all major stakeholders in sustainable groundwater management. Even so, the outcomes could not provide definitive answers to the problems nor could they provide accurate forecasts of policy impacts.

\subsubsection{Objective 3: Integrating perception-based knowledge from stakeholders with data-driven knowledge from empirical studies in policy option analysis}

In chapter 4 the potential of a new approach in FCM that incorporates both qualitative evidence (i.e. stakeholders' perceptions) and quantitative evidence 
(i.e. empirical data) into a SES model is introduced and analysed. This model was used to investigate to what extent the combination of these two data sources improves the quality of a model that relies solely on perception data. The findings indicate that combining the farmers' subjective knowledge with objective data of water scarcity in Rafsanjan is a useful approach to model policy scenarios for the Rafsanjan SES.

The comparison between policy-makers', farmers' and data-driven FCMs show that policy makers' perceptions in Rafsanjan are generally closer to measureddata than farmers' perceptions and in many cases farmers' perceptions have large difference with both data and policy makers' perceptions. This difference represents the knowledge gap between farmers and policy makers/researchers whose communication and collaboration is complementary for a proper understanding of the system. Moreover, the results of this chapter indicates that qualitative-quantitative FCM (mixed-FCM) is useful for the study of SESs in which some environmental changes are either gradual or invisible to stakeholders' direct observation e.g. groundwater level change and land subsidence. Whereas, for the study of environmental changes that can be perceived immediately by stakeholders over relatively short time frames, an FCM that solely relies on perception data would suffice. Finally, the results of policy option analysis with the mixed-FCM shows that the economic change policy, which aims at economic diversification of Rafsanjan communities, may have the most impact on the recovery of the whole system. The economic change policy option has the largest influence on increasing groundwater level, the regional economy and sustainable investment of the region and decreasing farmers' vulnerability and land subsidence.

\subsubsection{Objective 4: Simulating impacts of policy options by integrating multi-variables' behaviour and human interactions in SESs' modelling}

For effective policy simulation in SESs new simulation approaches are required that involve both human interactions and multi-variables' behaviour in SES modelling. Chapter 5 presents a step-wise methodology to integrate FCM and Agent-Based Modelling (ABM) methods for simulating impacts of policy options in SESs. FCM presents farmers' knowledge, perceptions, decisions and actions facing water scarcity in Rafsanjan, and ABM simulates spatial-temporal impacts of policies considering human interventions and dynamic interactions of socialecological variables. This integrated methodology considers features of complex SESs that cannot be fully covered by either modelling approach if used independently. These features consist of: causality, feedback mechanisms, social-spatial heterogeneity, and time scales.

The FCM-ABM methodology was used to compare outcomes of three policy options: shrinking farms, irrigation change and increased participation. 
Although shrinking farms is a high incentive policy for farmers to reduce their irrigation areas and thus decrease pressures on the aquifer, due to the high emigration of farmers in this scenario, it is an unsatisfactory policy from a socio-economic perspective. Rather, a policy to facilitate farmers' participation in the management and control of their groundwater use leads to the highest reduction in groundwater use, and it reduces emigration. Surprisingly, adapting new irrigation technologies does not have any significant impact on reducing overall groundwater use in the region.

\subsection{Reflections}

This section reflects on the key findings of this thesis. It consists of the summary of the main contributions and recommendations for the future studies.

\subsubsection{Main contributions}

This thesis contributes in six ways to the challenges of policy option analysis in SES.

First, it provides four policy support methods to address different problem contexts and policy objectives. DPSIR is a problem structuring framework that helps to categorize and understand different aspects of problem before policy making. Perceived-FCM is useful in predicting stakeholders' acceptance of the new policies. Mixed-FCM is useful in simulating and analysing impacts of policy options in the multi-factorial SESs, for which both subjective and objective knowledge are important in better understating the system's behaviour. Finally, FCM-based ABM is useful in simulating impacts of policy options by considering actual human decisions and actions as well as the multifactors' behaviour in the SESs. Each method has its own application, required resources, and ability of capturing complexity of systems. Figure 1 shows that as the captured complexity by methods increases, so does the required resources-e.g. time, money, data resources, etc.-for implementation as well. Therefore, though the FCM-based ABM captures the highest complexity of the systems (i.e. multi-factorial and multi-stakeholder behaviour of the system considering qualitative and quantitative evidence), it also consumes the most time, money and data resources. Nevertheless, not all SES decision making requires the full complexities of the system to be considered. In some policy simulations, a perceived-FCM based solely on stakeholders' perceptions might be sufficient (e.g. policy simulations that aim to evaluate the level of acceptance of policies by people, or to show policy makers how local stakeholders perceive a problem). In some cases, policy simulations aim to model and analyse the relation of social and ecological factors without dynamic interaction of stakeholders in the system e.g. impact of climate change on groundwater level or current groundwater consumption. In such cases, a 
mixed-FCM would suffice. Therefore, part of effectiveness of a policy analysis depends on choosing the right method tailored for problem contexts and policy objectives of each specific case.

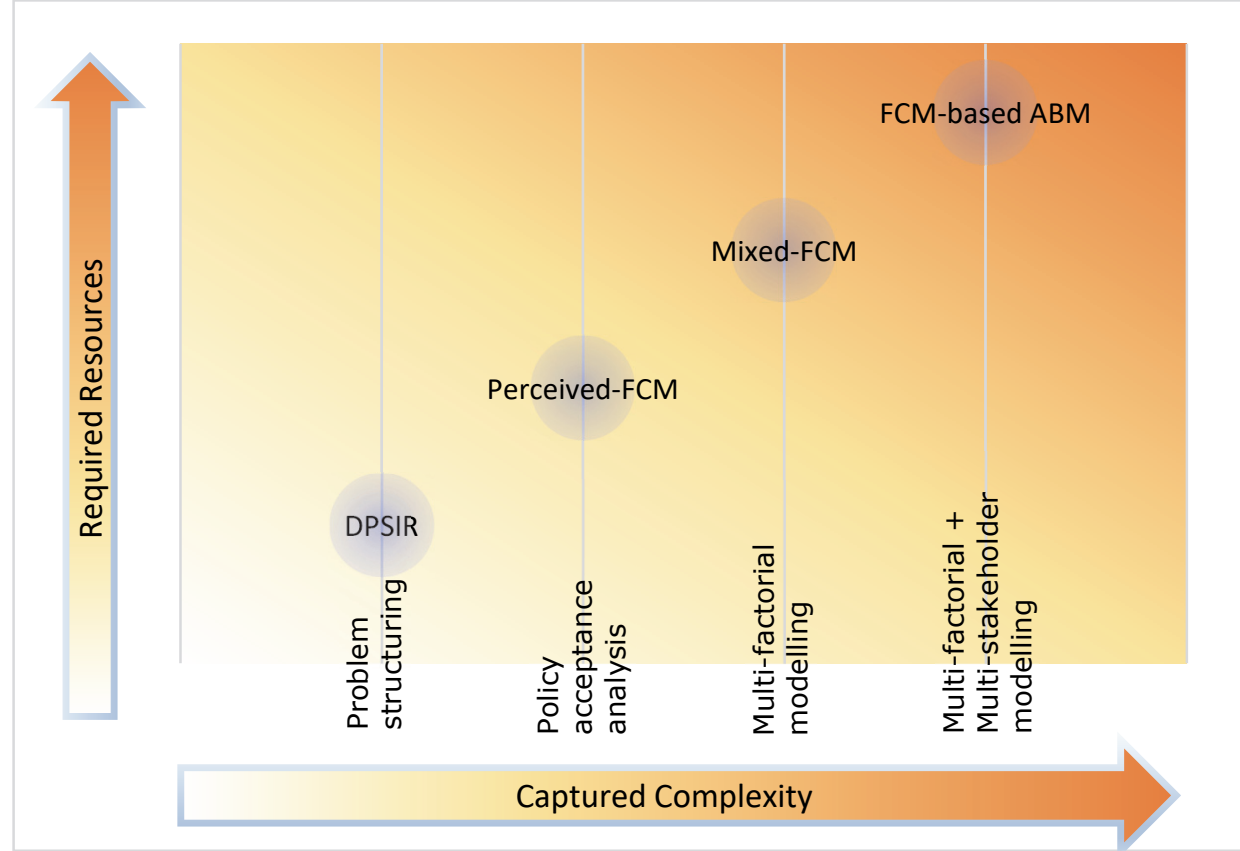

Figure 6.1: Four policy support methods, their required resources, and ability to capture complexity

The second major contribution of this study is in introducing methods that facilitate engaging people's knowledge, perceptions, decisions and actions together in the process of policy making. The need for incorporating such human elements in decision making has been supported by various fields related to SESs studies, such as system resilience, social learning, risk management, and decision support system (Janssen, 2002, Filatova et al., 2013). Their arguments can be summarized in two main points: First, policy making in SESs is influenced by the implicit and explicit ways that people think about how the world works or should work, and the impacts of different interventions on things they value i.e. people's knowledge and perceptions about the SESs (Argyris and Schön, 1997). Second, to influence resource dynamics, policies should simulate and impact resource-users' behaviour. It is known that managing resources in SESs is about managing the people's interventions rather than the resources directly (Ludwig et al., 1993). Therefore, policy makers need to understand and target the people's decisions and actions in relation to the natural resources. However, the lack of policy analysis methods that incorporate all these human elements has been recognized in the literature (Elsawah et al., 2015). In this study, chapter 3, 4, and 5 contributed in developing methods to incorporate 1) stakeholders' 
knowledge and perception, and 2) stakeholders' interactions and behaviour in response to the implementation of new policies.

The third major contribution of this research is in bridging the gap in integrated use of qualitative and quantitative evidence in SES modelling. Mathematical and computational models, are associated with quantitative data. On the other hand, qualitative evidence has often been seen as unscientific, too subjective, biased, unreliable and context-specific (Edmonds, 2010). However, there is now a new insight that "SES simulation models" can be highly complex and not necessarily fully numerically based. The required quantitative data to cover all aspects of a SES is often incomplete and/or difficult to access. Therefore, we can no longer ignore the important role of qualitative evidence and complementary role of qualitative and quantitative data in SESs' modelling. Nevertheless, there are still difficulties in integrating qualitative and quantitative data in simulation modelling (Moss and Edmonds, 2005, Edmonds, 2010). Chapter 4 and 5 of this study contributed in 1) integrating qualitative and quantitative data in modelling the system, and 2) using qualitative data in the computational and mathematical model, respectively.

The fourth major contribution of this thesis is in bridging the gap between micro-level and macro-level behaviour of the system. Among the simulation models used as policy support tool, system dynamic models-including FCMtake a macro-level view of the world and ABMs have a micro-level view. In fact, both simulation modelling approaches share a common aim: to understand the behaviour of dynamic systems and their underlying principles (Schieritz, 2002). Yet, system dynamic models look for these principles in the global system structure, whereas, an ABM seeks them in agent's behaviour rules. Therefore, system dynamics represent the world based on the macrolevel relationships among the system's variables, in which there is no place for individual behaviour of agents (e.g. stakeholders) that might affect variables' behaviour. On the other hand, ABM represents the behaviour at individual level and the global behaviour only emerges as a result of such individuals' behaviour. Although there have been some studies on integrating system dynamic models and ABM methods (Shafiei et al., 2013, Borshchev and Filippov, 2004) studies that combine FCM and ABM have, until now, been missing. In our study, $A B M$ provides a micro-level view at the individual farmers' dynamic behaviour and represent the emergent impact of their actions on overall groundwater use of their regions. The, FCM provides a macro-level view at the general system structure and represent the impacts of changing groundwater use on e.g. groundwater level, pistachio production, groundwater quality, land subsidence, etc., which might indirectly influence the behaviour of farmers.

The fifth contribution of this study is in bridging the gap between actor-based and factor-based approach in SES modelling. Most of the policy simulation 
models follow either of these approaches: 1) factor-based modelling approach e.g. System Dynamic Model, Fuzzy Cognitive Mapping and Bayesian Neural Network, that typically consists of sets of different equations that describe changes in system behaviour as a holistic function of changes in variables' (i.e. factors') states. These approaches miss the influence of human decisions and actions in the process of policy analysis. 2) Actor-based modelling approach, e.g. agent-based modelling, in which individuals are units of analysis and the model represents interacting behaviour of individuals i.e. actors. This approach often lacks the actual justification for the actors' behaviour and impact of their behaviour on the broader environment. This study fills a gap by combining FCM and $A B M$ to model the influence of both variables' behaviour and agents' interactions in a SES.

The final contribution of this research is introducing policy support methods that cover the main features of a complex SES with wicked problems. Such features include causality, feedback loops, heterogeneity and time scale. Causality in SESs refers to causal relationships between human actions and their surrounding social-ecological factors i.e. economic situation, climate variables, access to natural resources, etc. Feedback loops in SES structure occurs when output from one social or ecological factor eventually influence input to that same factor-said as the system feeds back into itself. Individual heterogeneity refers to various types of involved stake-holders in SESs and highlights their different preferences, available actions and long-term goals (Macy and Willer, 2002). Moreover, spatial heterogeneity refers to the various environmental properties in different locations (Filatova et al., 2013). Due to individual and spatial heterogeneities, a policy impact may vary in different locations and on different individuals, which calls for attention on social as well as spatial differences for policy simulation in SESs. Finally, time scale in SESs refers to the timing of different variables' changes and agents' actions. For example, slowly changing variables (e.g. population change) versus fast changing variables (e.g. government policies) or high frequency actions (e.g. farm irrigation) and low frequency actions (e.g. buying lands). Therefore, policy making in SESs has to consider all these features for a holistic policy option analysis prior to their implementation in the real world. Our integrated methodological approach for policy simulation could cover all these four features of the SES. FCM represents the causal relationship between human actions and environmental factors' changes, and explicitly incorporate feedback in human-environment interactions. ABM incorporates individual and spatial heterogeneity as well as temporal aspect of the SES.

Finally, the outcomes will be useful for three main groups: 1. researchers who might benefit from novel methods to model SESs, 2 . practitioners who can use the methods for participatory policy making, and 3. students who can learn 
from step-wise instructions developed for all objectives of this research in their future studies.

\subsubsection{Recommendations for future research}

During completing this research some limitations have been faced and several further research ideas have been evolved that could be addressed in future studies.

Policy design and implementation by stakeholders: The methods to involve stakeholders in the process of decision making for SESs are mainly developed for three main objectives. First, policy co-design which is the process of understanding the problems and generating solutions jointly with stakeholders (Blomkamp, 2018, Durose and Richardson, 2015). Therefore, in these approaches participation of stakeholders are used to promote and articulate the innovative and user friendly decisions, i.e. co-designed policies (Voinov and Bousquet, 2010). Second, policy co-analysis which is the process of extracting knowledge, preferences and perceptions of stakeholders to analyse the effectiveness of predesigned solutions or policies; whether it brings about the desirable outcomes or not. In these approaches, participation of stakeholders is used as input material for simulating the potential impacts of policies before their implementation. Third, policy co-implementation, which is the process of integrating stakeholders in policies' implementation. Clearly, policy implementation is not a single-shot action nor process, but rather a continual implementation and evaluation loop. Thus, during the policy implementation process we should be prepared for unexpected changes in goals, priorities, and conditions, and be ready to adjust accordingly. Therefore, in these approaches the feedbacks and responses of stakeholders are used to evaluate the impact of previous policies before implementing the next ones.

In this study we demonstrated applicability of our methodology for policy analysis i.e. potential impact of future policies and policy implementation i.e. responses of stakeholders to the executed policies. Yet, applicability of these methods for policy co-design requires further studies.

Participatory validation of policy impact results: In our research we validated our models' function-whether they reflect current or past situation of the real system-with stakeholders' inputs (in FCM model) and time series data (in ABM model). However, validating the impacts of policies, particularly those that have not been implemented before, is a long run process, and thus cannot be validated with current data and knowledge. Therefore, we suggest validation of such policies' implementation results after implementation of one of the policies. 
Usefulness of methodologies for policy makers: An ultimate goal of this study is to provide useful tools to support policy makers and practitioners in their decision-making processes. Thus, policy makers should be able and willing to use them. An extension of this research would be a study on usefulness of introduced methods for the policy makers' decision-making in real world practices. Usefulness of methods is defined as a function of two quality parameters: utility plus usability (Nielsen, 1994). By utility here we mean the question of whether the method does what policy makers want-in solving the SES problems-, and by usability we mean the ease of use and learnability of methods by policy makers or practitioners - whether they understand and operate the method efficiently. These two qualities can be evaluated by policy makers in different contexts and SES problems.

Generalizability of methods: We argued that methods developed in this study-i.e. perceived-FCM, Mixed-FCM, and FCM-based ABM-can be generally used by policy makers in social-ecological and social-technical systems i.e. where 1) both qualitative and quantitative evidences are crucial in understanding the system, and/or 2) both human's and environmental factors' behaviour should be considered in policy making. However, in different research problems, the methods should be tailored to suit with the contextual needs of that specific project. For example, choosing between individual or group interview, relying on either qualitative or quantitative data for different parameters, considering which aspects of human behaviour e.g. learning, prediction, sensing, etc., and in general the question of how much complexity is enough for modelling the system, all are context specific and problem specific issues. Therefore, more studies are needed to investigate application of these methods in different research domains-e.g. energy, fishery, flood risk-and contexts-e.g. different cultures and social-political situations.

Social, ecological and political externalities: In this study, we have dismissed the possible social, ecological and political externalities in simulating future scenarios. For instance, we did not consider possible future externalities such as sanctions against Iran, an economic crisis, and new development policies nor new trends of climate change and natural resource change e.g. increase of rainfalls and groundwater level. Yet, these external variables can also be considered by defining them in scenarios. However, it requires more insights and imposes an even higher complexity to the model, which was beyond the scope and duration of this study.

Non-experienced policies: In this research, the stakeholders' knowledge and perceptions were the main inputs, and historical data was secondary input to the simulation models. Therefore, the simulations are based on whatever has been experienced in the past i.e. policies that have been implemented or their impacts have been experienced in other ways. For instance, the impact 
of shrinking farms policy could be simulated because farmers had experienced reducing their farms area and its social-ecological impacts on their farms. Yet, they have not experienced new policies such as the water transfer projectsi.e. desalination and pumping water from Persian gulf to arid areas-or a new water pricing policy. Therefore, impacts of such policies could not be simulated by reliance on stakeholders' experiences and perception in this study. As a result, more studies are needed to use public engagement in the process of analysing new policies that have not been experienced by stakeholders. One approach might be using Stated Choice studies, in which stakeholders explain what they would be likely to do under new circumstances.

Human learning and prediction: There are situations, in which people's behaviour or people's choices depends on the behaviour or the choices of other people. These situations usually do not permit any simple summation or extrapolation to the aggregates without communication and learning among agents (Schelling, 2006). In the presented model in the chapter 5 we employed an indirect communication approach to reflect the learning process that may emerge within a SES. This is, members of the community learn through the process of observing changes in the environment. For instance, when agent " $A$ " performs an action " $\mathrm{X}$ " other agents learn about it through the resulted changes in the environment-and not through direct communication i.e. through an assertion from agent A toward agent B. Although this approach provides significant insights on how a SES evolves, modelling the collective behaviours that may emerge in societies requires capturing direct communication among agents in a systematic manner. In principle, a communication action, performed by agent $A$, informs other agents about the mental status of A (e.g. its preferences, beliefs, or intentions). This way we can analyse and more precisely describe the behaviour of SESs and their potential responses to interventions. This can be achieved by using the reach literature on neural networks, evolutionary algorithms, or other learning techniques to allow realistic learning and adaptation (Hare and Deadman, 2004, Bonabeau, 2002, Marchiori and Warglien, 2008). 


\section{Bibliography}

Abdullah, L., Khadiah S., 2011. Fuzzy linguistic for measuring customer satisfaction. Int. J. Latest Trends Comput. 2,

Adger, W.N., Arnell N.W., Tompkins E.L., 2005. Successful adaptation to climate change across scales. Global environmental change. 15, 77-86

Adger, W.N., Dessai S., Goulden M., Hulme M., Lorenzoni I., Nelson D.R., Naess L.O., Wolf J., Wreford A., 2009. Are there social limits to adaptation to climate change? Climatic change. 93, 335-354

Agah, M., Hassani M., 2014. Water rights in Iranian plateau. Water Reaserach Institure of Iran, Tehran, Iran

Al-Doski, J., Mansor S.B., Shafri H.Z.M., 2013. NDVI Differencing and Postclassification to Detect Vegetation Changes in Halabja City, Iraq. IOSR Journal of Applied Geology and Geophysics (IOSR-JAGG). 1, 01-10

Alasti, S. 2013. Legislation on Use of Water in Agriculture: Iran. washington, DC: The Library of Congress.

Alizadeh, A., Keshavarz A., 2005. Status of agricultural water use in Iran, in: Water Conservation, Reuse, and Recycling: Proceedings of an IranianAmerican Workshop. Washington DC, USA, 2005. National Academies Press, pp 94-105

An, L., 2012. Modeling human decisions in coupled human and natural systems: Review of agent-based models. Ecol. Model. 229, 25-36. doi:10.1016/j.ecolmodel.2011.07.010

Ansell, C., Gash A., 2008. Collaborative governance in theory and practice. Journal of public administration research and theory. 18, 543-571

Arcidiacono, C., Procentese F., Napoli I.D., 2009. Qualitative and quantitative research: An ecological approach. International Journal of Multiple Research Approaches. 3, 163-176

Argyris, C., Schön D.A., 1997. Organizational learning: A theory of action perspective. Reis, 345-348

Ascough, J., Maier H., Ravalico J., Strudley M., 2008. Future research challenges for incorporation of uncertainty in environmental and ecological decision-making. Ecological Modelling. 219, 383-399

Atkins, J.P., Gregory A.J., Burdon D., Elliott M., 2011. Managing the marine environment: is the DPSIR framework holistic enough? Systems Research and Behavioral Science. 28, 497-508

Basco-Carrera, L., Warren A., Van Beek E., Jonoski A., Giardino A., 2017. Collaborative modelling or participatory modelling? A framework for water resources management. Environmental Modelling \& Software. 91, 95-110. doi:10.1016/j.envsoft.2017.01.014

Baykasoglu, A., Durmusoglu Z.D., Kaplanoglu V., 2011. Training fuzzy cognitive maps via extended great deluge algorithm with applications. Computers in Industry. 62, 187-195 
Bell, S., 2012. DPSIR= A problem structuring method? An exploration from the "Imagine" approach. European Journal of Operational Research. 222, 350-360

Berkes, F., Folke C., Colding J., 2000. Linking social and ecological systems: management practices and social mechanisms for building resilience. Cambridge University Press,

Bersini, H., 2012. Uml for abm. J. Artif. Soc. Soc. Simul. 15, 9. doi: $10.18564 /$ jasss. 1897

Biggs, R., Rhode C., Archibald S., Kunene L.M., Mutanga S.S., Nkuna N., Ocholla P.O., Phadima L.J., 2015. Strategies for managing complex social-ecological systems in the face of uncertainty: examples from South Africa and beyond. Ecology and Society. 20,

Binder, C.R., Hinkel J., Bots P.W., Pahl-Wostl C., 2013. Comparison of frameworks for analyzing social-ecological systems. Ecology and Society. 18, 26

Blomkamp, E., 2018. The Promise of Co-Design for Public Policy. Australian Journal of Public Administration. 00, 1-15. doi:https://doi.org/10.1111/1467-8500.12310

Bonabeau, E., 2002. Agent-based modeling: Methods and techniques for simulating human systems. Proceedings of the National Academy of Sciences. 99, 7280-7287

Borja, Á., Galparsoro I., Solaun O., Muxika I., Tello E.M., Uriarte A., Valencia V., 2006. The European Water Framework Directive and the DPSIR, a methodological approach to assess the risk of failing to achieve good ecological status. Estuarine, Coastal and Shelf Science. 66, 84-96

Borshchev, A., Filippov A., 2004. From system dynamics and discrete event to practical agent based modeling: reasons, techniques, tools, in: Proceedings of the 22nd international conference of the system dynamics society. 2004. Citeseer,

Bousquet, F., Barreteau O., D'aquino P., Etienne M., Boissau S., Aubert S., Le Page C., Babin D., Castella J.-C., 2002. Multi-agent systems and role games: collective learning processes for ecosystem management. Complexity and ecosystem management: The theory and practice of multi-agent systems, 248-285

Buruzs, A., Hatwágner M.F., Kóczy L.T., 2015. Expert-Based Method of Integrated Waste Management Systems for Developing Fuzzy Cognitive Map, in: Complex System Modelling and Control Through Intelligent Soft Computations. Springer, pp 111-137

Campo, P., Bousquet F., Villanueva T., 2010. Modelling with stakeholders within a development project. Environmental Modelling \& Software. 25, $1302-1321$

Caponera, D.A., Nanni M., 1992. Principles of Water Law and Administration: National and International 2nd edition, revised and updated by Marcella Nanni, vol 1. CRC Press, 
Carvalho, J.P., 2013. On the semantics and the use of fuzzy cognitive maps and dynamic cognitive maps in social sciences. Fuzzy Sets and Systems. 214, 6-19. doi:10.1016/j.fss.2011.12.009

Carvalho, J.P., Tomè J.A., 2000. Rule based fuzzy cognitive maps-qualitative systems dynamics, in: Fuzzy Information Processing Society, 2000. NAFIPS. 19th International Conference of the North American. 2000. IEEE, pp 407-411. doi: 10.1109/NAFIPS.2000.877462

Castella, J.-C., Trung N., Boissau S., 2005. Participatory simulation of land-use changes in the northern mountains of Vietnam: the combined use of an agent-based model, a role-playing game, and a geographic information system. Ecol. Soc. 10, 1-32. doi:10.5751/es-01328-100127

Castelletti, A., Soncini-Sessa R., 2007. Bayesian Networks and participatory modelling in water resource management. Environmental Modelling \& Software. 22, 1075-1088

Chapin Iii, F.S., Robards M.D., Huntington H.P., Johnstone J.F., Trainor S.F., Kofinas G.P., Ruess R.W., Fresco N., Natcher D.C., Naylor R.L., 2006. Directional changes in ecological communities and social-ecological systems: a framework for prediction based on Alaskan examples. The American Naturalist. 168, S36-S49

Council, N.R., 2008. The promises and perils of participation, in: Thomas Dietz, PCS (Ed) Public Participation in Environmental Assessment and Decision Making The National Academies Press, Washinton, D.C, USA, pp 33-74. doi:https://doi.org/10.17226/12434

Cumming, G.S., Cumming D.H., Redman C.L., 2006. Scale mismatches in social-ecological systems: causes, consequences, and solutions. Ecology and Society. 11,

David, R. 1996. An Empirical Analysis of Dutch Disease: Developing and Developed Countries.

Davies, H.T., Nutley S.M., 2000. What works?: Evidence-based policy and practice in public services. Policy Press,

Dehghani, M., Rastegarfar M., Ashrafi R.A., Ghazipour N., Khorramrooz H.R., 2014. Interferometric SAR and Geospatial Techniques Used for Subsidence Study in the Rafsanjan Plain. American Journal of Environmental Engineering. 4, 32-40

Dickerson, J.A., Kosko B., 1994. Virtual worlds as fuzzy cognitive maps. Presence: Teleoperators \& Virtual Environments. 3, 173-189

Durning, D., 1993. Participatory policy analysis in a social service agency: A case study. Journal of Policy Analysis and Management. 12, 297-322

Durning, D., 1999. Debating technologies: a methodological contribution to the design and evaluation of participatory policy analysis. Journal of Policy Analysis and Management: The Journal of the Association for Public Policy Analysis and Management. 18, 339-343

Durose, C., Richardson L., 2015. Designing public policy for co-production: theory, practice and change. Policy Press, 
Edmonds, B., 2010. Computational modelling and social theory-The dangers of numerical representation. Computational Analysis of Firm Organisations and Strategic Behaviour, 36-68

Edmonds, B., Moss S., 2004. From KISS to KIDS-an 'anti-simplistic'modelling approach, in: International workshop on multi-agent systems and agentbased simulation. 2004. Springer, pp 130-144

Eea 1999. Environmental indicators: Typology and overview. European Environment Agency Copenhagen.

Elsawah, S., Guillaume J.H., Filatova T., Rook J., Jakeman A.J., 2015. A methodology for eliciting, representing, and analysing stakeholder knowledge for decision making on complex socio-ecological systems: From cognitive maps to agent-based models. J. Environ. Manage. 151, 500-516. doi:doi:10.1016/j.jenvman.2014.11.028

Fairweather, J., 2010. Farmer models of socio-ecologic systems: Application of causal mapping across multiple locations. Ecological Modelling. 221, 555-562

Faostat 2015. FAOSTAT online database. doi:http://faostat3.fao.org/compare/E

Farzanegan, M.R., Markwardt G., 2009. The effects of oil price shocks on the Iranian economy. Energy Economics. 31, 134-151

Filatova, T., Verburg P.H., Parker D.C., Stannard C.A., 2013. Spatial agentbased models for socio-ecological systems: Challenges and prospects. Environ. Modell. Softw. 45, 1-7. doi:10.1016/j.envsoft.2013.03.017

Folke, C., 2006. Resilience: The emergence of a perspective for socialecological systems analyses. Global Environmental Change. 16, 253-267

Gari, S.R., Newton A., Icely J.D., 2015. A review of the application and evolution of the DPSIR framework with an emphasis on coastal socialecological systems. Ocean \& Coastal Management. 103, 63-77

Geurts, J.L., Joldersma C., 2001. Methodology for participatory policy analysis. European Journal of Operational Research. 128, 300-310

Ghorbani, A., Dijkema G., Schrauwen N., 2015. Structuring qualitative data for agent-based modelling. J. Artif. Soc. Soc. Simul. 18, 2. doi: $10.18564 /$ jasss. 2573

Giabbanelli, P.J., Gray S.A., Aminpour P., 2017. Combining fuzzy cognitive maps with agent-based modeling: Frameworks and pitfalls of a powerful hybrid modeling approach to understand human-environment interactions. Environ. Modell. Softw. 95, 320-325. doi:10.1016/j.envsoft.2017.06.040

Gilanpour, O., 2006. Challenges of Iran's agriculture sector in accession Process to the WTO, in: Proceeding of Eighth European Trade Study Meeting, Vienna. On http://www.etsg.org/ETSG2006/papers/Gilanpour.pdf. 2006.

Gilbert, N., 2008. Agent-based models. vol 153. Sage, 
Gilson, L., 2014. Qualitative research synthesis for health policy analysis: what does it entail and what does it offer? Health policy and planning. 29, iii1iii5

Giordano, R., Passarella G., Uricchio V., Vurro M., 2007. Integrating conflict analysis and consensus reaching in a decision support system for water resource management. Journal of Environmental Management. 84, 213228

Gohari, A., Eslamian S., Mirchi A., Abedi-Koupaei J., Bavani A.M., Madani K., 2013. Water transfer as a solution to water shortage: a fix that can backfire. Journal of Hydrology. 491, 23-39

Gopinath, G., Ambili G., Gregory S.J., Anusha C., 2015. Drought risk mapping of south-western state in the Indian peninsula-A web based application. Journal of Environmental Management,

Gosselin, F., Cordonnier T., Bilger I., Jappiot M., Chauvin C., Gosselin M., 2018. Ecological research and environmental management: We need different interfaces based on different knowledge types. J. Environ. Manage. 218, 388-401. doi:10.1016/j.jenvman.2018.04.025

Gray, S., Chan A., Clark D., Jordan R., 2012. Modeling the integration of stakeholder knowledge in social-ecological decision-making: benefits and limitations to knowledge diversity. Ecological Modelling. 229, 88-96

Gray, S.A., Gray S., De Kok J.L., Helfgott A.E., O'dwyer B., Jordan R., Nyaki A., Analyzing F., 2015. Using fuzzy cognitive mapping as a participatory approach to analyze change, preferred states, and perceived resilience of social-ecological systems. Ecol. Soc. 20, 11

Gray, S.A., Zanre E., Gray S., 2014. Fuzzy cognitive maps as representations of mental models and group beliefs, in: Fuzzy cognitive maps for applied sciences and engineering. Springer, pp 29-48. doi:10.1007/978-3-64239739-4_2

Gregory, A.J., Atkins J.P., Burdon D., Elliott M., 2013. A problem structuring method for ecosystem-based management: The DPSIR modelling process. European Journal of Operational Research. 227, 558-569

Grimm, V., Berger U., Deangelis D.L., Polhill J.G., Giske J., Railsback S.F., 2010. The ODD protocol: a review and first update. Ecol. Model. 221, 2760-2768. doi:10.1016/j.ecolmodel.2010.08.019

Grimm, V., Polhill G., Touza J., 2017. Documenting social simulation models: the ODD protocol as a standard, in: Simulating Social Complexity. Springer, pp 349-365. doi:10.1007/978-3-540-93813-2_7

Groeneveld, J., Müller B., Buchmann C.M., Dressler G., Guo C., Hase N., Hoffmann F., John F., Klassert C., Lauf T., 2017. Theoretical foundations of human decision-making in agent-based land use models-A review. Environ. Modell. Softw. 87, 39-48. doi:10.1016/j.envsoft.2016.10.008

Hare, M., Deadman P., 2004. Further towards a taxonomy of agent-based simulation models in environmental management. Mathematics and Computers in Simulation. 64, 25-40 
Hatwagner, M.F., Yesil E., Dodurka F., Papageorgiou E.I., Urbas L., Koczy L.T., 2018. Two-stage learning based fuzzy cognitive maps reduction approach. IEEE Trans Fuzzy Syst,

Henriksen, H.J., Rasmussen P., Brandt G., Von Buelow D., Jensen F.V., 2007. Public participation modelling using Bayesian networks in management of groundwater contamination. Environmental Modelling \& Software. 22, 1101-1113

Hermans, L.M., Thissen W.A., 2009. Actor analysis methods and their use for public policy analysts. European Journal of Operational Research. 196, 808-818

Himanshu, S., Singh G., Kharola N., 2015. Monitoring of Drought using Satellite Data. International Research Journal of Earth Sciences. 3, 6672

Holland, J., Blackburn J., Chambers R., 1998. Whose voice? The Intermediate Technology Publications,

Holling, C.S., Gunderson L.H. 2002. Resilience and adaptive cycles. In: Panarchy: Understanding Transformations in Human and Natural Systems, 25-62. Washington, D.C., USA: Island press.

Hsieh, H.-F., Shannon S.E., 2005. Three approaches to qualitative content analysis. Qualitative Health Research. 15, 1277-1288

Ipcc 2007. Climate Change 2007: Working Group II: Impacts, Adaptation and Vulnerability.

Irvin, R.A., Stansbury J., 2004. Citizen participation in decision making: is it worth the effort? Public administration review. 64, 55-65

Iwpri 2014. Ground water resources sustainability: Case study of Rafsanjan. In: Institute, IWR (ed.). Tehran, Iran.

Jamab 2011. Upgrading Study of Water Comprehensive Plan. Tehran, Iran: Madar Consultant, Water Research department.

Janssen, M., 2002. Complexity and ecosystem management: the theory and practice of multi-agent systems. Edward Elgar Publishing,

Jetter, A.J., Kok K., 2014. Fuzzy Cognitive Maps for futures studies-A methodological assessment of concepts and methods. Futures. 61, 4557. doi:10.1016/j.futures.2014.05.002

Johnson, J., Ormerod P., Rosewell B., Nowak A., Zhang Y.-C., 2017. NonEquilibrium Social Science and Policy, in: Non-Equilibrium Social Science and Policy. Springer, pp 1-17

Kahan, J.P., 2001. Focus groups as a tool for policy analysis. Analyses of Social Issues and Public Policy. 1, 129-146

Kalantari, I. 2015. The environmental failure in protecting lagoons. In: Jamshidi, M (ed.). Tehran, Iran: Etemad newspaper.

Kerman Provincial Government 2014. Policie and directives on protection and conservation of groundwater aquifers. In: Affairs, D (ed.). Kerman, Iran.

Khorami, A.R., Pierof S., 2013. The Role of Agriculture in Iran's Economic Development. American Economic Review. 92, 160-164 
Kok, K., 2009. The potential of Fuzzy Cognitive Maps for semi-quantitative scenario development, with an example from Brazil. Global Environmental Change. 19, 122-133

Kontogianni, A.D., Papageorgiou E.I., Tourkolias C., 2012. How do you perceive environmental change? Fuzzy Cognitive Mapping informing stakeholder analysis for environmental policy making and non-market valuation. Applied Soft Computing. 12, 3725-3735

Kosko, B., 1986a. Fuzzy cognitive maps. Int. J. Man. Mach. Stud. 24, 65-75

Kosko, B., 1986b. Fuzzy knowledge combination. International Journal of Intelligent Systems. 1, 293-320

Kosko, B., 1987. Adaptive inference in fuzzy knowledge networks, in: Proc. 1st Int. Conf. Neural Networks. 1987. pp 261-268

Kosko, B., 1993. Fuzzy thinking: The new science of fuzzy logic. Hyperion Books,

Kristensen, P., 2004. The DPSIR framework. National Environmental Research Institute, Denmark, 10

Kulsum, U., Timmermans J., Haasnoot M., Khan M.S.A., Thissen W., Middelkoop H., Van Beek E., 2017. Modelling of farmers' livelihood desicion-making to understand their adaptation response to changing conditions in southwest coastal Bangladesh in the Ganges delta. 6th International Conference on Water \& Flood Management

Langmead, O., Mcquatters-Gollop A., Mee L.D., Friedrich J., Gilbert A.J., Gomoiu M.-T., Jackson E.L., Knudsen S., Minicheva G., Todorova V., 2009. Recovery or decline of the northwestern Black Sea: A societal choice revealed by socio-ecological modelling. Ecological Modelling. 220, 2927-2939

Lavin, E.A., Giabbanelli P.J., 2017. Analyzing and simplifying model uncertainty in fuzzy cognitive maps, in: Simulation Conference (WSC), 2017 Winter. 2017. IEEE, pp 1868-1879

Lavin, E.A., Giabbanelli P.J., Stefanik A.T., Gray S.A., Arlinghaus R., 2018. Should we simulate mental models to assess whether they agree?, in: Proceedings of the Annual Simulation Symposium. 2018. Society for Computer Simulation International, $\mathrm{p} 6$

Levin, S., Xepapadeas T., Crépin A.-S., Norberg J., De Zeeuw A., Folke C., Hughes T., Arrow K., Barrett S., Daily G., 2013. Social-ecological systems as complex adaptive systems: modeling and policy implications. Environ. Dev. Econ. 18, 111-132. doi:doi: $10.1017 /$ S1355770X12000460

Lewison, R.L., Rudd M.A., Al-Hayek W., Baldwin C., Beger M., Lieske S.N., Jones C., Satumanatpan S., Junchompoo C., Hines E., 2016. How the DPSIR framework can be used for structuring problems and facilitating empirical research in coastal systems. Environmental Science \& Policy. $56,110-119$ 
Liu, J., Chi Y., Zhu C., Jin Y., 2017. A time series driven decomposed evolutionary optimization approach for reconstructing large-scale gene regulatory networks based on fuzzy cognitive maps. BMC Bioinformatics. 18,241

Liu, J., Dietz T., Carpenter S.R., Alberti M., Folke C., Moran E., Pell A.N., Deadman P., Kratz T., Lubchenco J., 2007. Complexity of coupled human and natural systems. Science. 317, 1513-1516

López-Ridaura, S., Masera O., Astier M., 2002. Evaluating the sustainability of complex socio-environmental systems. The MESMIS framework. Ecological indicators. 2, 135-148

Lowe, C.D., Gilbert A.J., Mee L.D., 2014. Human-environment interaction in the Baltic Sea. Marine Policy. 43, 46-54

Ludwig, D., Hilborn R., Walters C., 1993. Uncertainty, resource exploitation, and conservation: lessons from history. Ecological Applications, 548-549

Lynam, T., De Jong W., Sheil D., Kusumanto T., Evans K., 2007. A review of tools for incorporating community knowledge, preferences, and values into decision making in natural resources management. Ecology and society. 12,

Macy, M.W., Willer R., 2002. From factors to actors: computational sociology and agent-based modeling. Annu. Rev. Sociol. 28, 143-166. doi:10.1146/annurev.soc.28.110601.141117

Madani, K., 2014. Water management in Iran: what is causing the looming crisis? Journal of Environmental Studies and Sciences, 1-14

Malena, C., 2004. Strategic partnership: challenges and best practices in the management and governance of multi-stakeholder partnerships involving UN and civil society actors, in: Multi-Stakeholder Partnerships and UN-Civil Society Relationships. Collection of Materials from the MultiStakeholder Workshop on Partnerships and UN-Civil Society Relationships. 2004. pp 32-63

Marchiori, D., Warglien M., 2008. Predicting human interactive learning by regret-driven neural networks. Science. 319, 1111-1113

Matmir, S., Reckien D., Flacke J., 2017. What do New Yorkers Think about Impacts and Adaptation to Heat Waves? An Evaluation Tool to Incorporate Perception of Low-Income Groups into Heat Wave Adaptation Scenarios in New York City. ISPRS International Journal of Geo-Information. 6,

Maxim, L., Spangenberg J.H., O'connor M., 2009. An analysis of risks for biodiversity under the DPSIR framework. Ecological Economics. 69, 1223

Mckenzie, B. 2005. Conversation Mapping Available: http://cognitiveedge.com [Accessed June 1, 2005].

Mease, L.A., Erickson A., Hicks C., 2018. Engagement takes a (fishing) village to manage a resource: Principles and practice of effective stakeholder 
engagement. J. Environ. Manage. 212, 248-257. doi: 10.1016/j.jenvman.2018.02.015

Mehryar, S., Sliuzas R., Sharifi A., Reckien D., Van Maarseveen M., 2017. A structured participatory method to support policy option analysis in a social-ecological system. J. Environ. Manage. 197, 360-372. doi:doi:10.1016/j.jenvman.2017.04.017

Mehryar, S., Sliuzas R., Sharifi A., Van Maarseveen M., 2015. The water crisis and socio-ecological development profile of Rafsanjan Township, Iran. WIT Trans. Ecol. Environ. . 199, 271. doi:doi:10.2495/RAV150231

Mehryar, S., Sliuzas R., Sharifi A., Van Maarseveen M., 2016. The socioecological analytical framework of water scarcity in Rafsanjan Township, Iran. International Journal of Safety and Security Engineering. 6, 764776. doi: 10.2495/SAFE-V6-N4-764-776

Metz, H.C., 2013. Iran, a country study. Government Printing Office,

Moazedi, A., Taravat M., Jahromi H.N., Madani K., Rashedi A., Rahimian S., 2011. Energy-water meter: a novel solution for groundwater monitoring and management, in: Proceedings of the World Environmental and Water Resources Congress. 2011. pp 962-969

Monnikhof, R.a.H. 2006. Policy analysis for participatory policy making. TU Delft, Delft University of Technology.

Moss, S., Edmonds B., 2005. Sociology and simulation: Statistical and qualitative cross-validation. American Journal of Sociology. 110, 10951131

Motagh, M., Shamshiri R., Haghighi M.H., Wetzel H.-U., Akbari B., Nahavandchi H., Roessner S., Arabi S., 2017. Quantifying groundwater exploitation induced subsidence in the Rafsanjan plain, southeastern Iran, using InSAR time-series and in situ measurements. Engineering Geology. 218, 134-151

Motagh, M., Walter T.R., Sharifi M.A., Fielding E., Schenk A., Anderssohn J., Zschau J., 2008. Land subsidence in Iran caused by widespread water reservoir overexploitation. Geophysical Research Letters. 35,

Mourhir, A., Papageorgiou E. 2017. Empirical comparison of fuzzy cognitive maps and dynamic rule-based fuzzy cognitive maps. In: Westphall, CB (ed.) ICAS 2017 Barcelona, Spain.

Mourhir, A., Papageorgiou E.I., Kokkinos K., Rachidi T., 2017. Exploring Precision Farming Scenarios Using Fuzzy Cognitive Maps. Sustainability. 9, 1241

Mourhir, A., Rachidi T., Papageorgiou E.I., Karim M., Alaoui F.S., 2016. A cognitive map framework to support integrated environmental assessment. Environmental Modelling \& Software. 77, 81-94

Mpo 2003. Comprehensive study of climat adaptibility. In: Company, IWRM (ed.). Tehran, Iran: Management and Planning Organization.

Müller, B., Bohn F., Dreßler G., Groeneveld J., Klassert C., Martin R., Schlüter M., Schulze J., Weise H., Schwarz N., 2013. Describing human decisions 
in agent-based models-ODD+ D, an extension of the ODD protocol. Environ. Modell. Softw. 48, 37-48. doi:10.1007/s10584-014-1275-0

Nakamura, K., Iwai S., Sawaragi T., 1982. Decision support using causation knowledge base. IEEE Transactions on Systems, Man, and Cybernetics. $12,765-777$

Namaalwa, S., Funk A., Ajie G., Kaggwa R., 2013. A characterization of the drivers, pressures, ecosystem functions and services of Namatala wetland, Uganda. Environmental Science \& Policy. 34, 44-57

Nielsen, J., 1994. Usability engineering. Elsevier,

Niemeijer, D., De Groot R.S., 2008. Framing environmental indicators: moving from causal chains to causal networks. Environment, Development and Sustainability. 10, 89-106

Nuno, A., Bunnefeld N., Milner-Gulland E.J., 2014. Managing social-ecological systems under uncertainty: implementation in the real world. Ecology and Society. 19,

O'donoghue, T., Punch K., 2003. Qualitative Educational Research in Action: Doing and Reflecting. Routledge,

Olazabal, M., Chiabai A., Foudi S., Neumann M.B., 2018. Emergence of new knowledge for climate change adaptation. Environmental Science \& Policy. 83, 46-53

Olazabal, M., Pascual U., 2015. Use of fuzzy cognitive maps to study urban resilience and transformation. Environmental Innovation and Societal Transitions,

Olazabal, M., Reckien D., 2015. 7 Fuzzy cognitive mapping: applications to urban environmental decision-making. Handbook of Research Methods and Applications in Environmental Studies, 148

Olsson, P., Folke C., Berkes F., 2004. Adaptive comanagement for building resilience in social-ecological systems. Environmental Management. 34, 75-90

Ostrom, E., 2007. A diagnostic approach for going beyond panaceas. Proceedings of the National Academy of Sciences. 104, 15181-15187

Ostrom, E., 2009. A general framework for analyzing sustainability of socialecological systems. Science. 325, 419-422

Özesmi, U., Özesmi S., 2003. A participatory approach to ecosystem conservation: fuzzy cognitive maps and stakeholder group analysis in Uluabat Lake, Turkey. Environmental Management. 31, 0518-0531

Özesmi, U., Özesmi S.L., 2004. Ecological models based on people's knowledge: a multi-step fuzzy cognitive mapping approach. Ecol. Model. . 176, 43-64. doi:doi:10.1016/j.ecolmodel.2003.10.027

Pak, M.V., Brieva D.C., 2010. Designing and implementing a role-playing game: a tool to explain factors, decision making and landscape transformation. Environmental Modelling \& Software. 25, 1322-1333

Papageorgiou, E., Kontogianni A., 2012. Using fuzzy cognitive mapping in environmental decision making and management: a methodological 
primer and an application, in: International Perspectives on Global Environmental Change. InTech. doi:10.5772/29375

Papageorgiou, E.I., 2012. Learning algorithms for fuzzy cognitive maps-a review study. IEEE Transactions on Systems, Man, and Cybernetics, Part C (Applications and Reviews). 42, 150-163

Papageorgiou, E.I., Hatwágner M.F., Buruzs A., Kóczy L.T., 2016. A Concept Reduction Approach for Fuzzy Cognitive Map Models in Decision Making and Management. Neurocomputing,

Papageorgiou, E.I., Poczęta K., Laspidou C., 2015. Application of fuzzy cognitive maps to water demand prediction, in: Fuzzy Systems (FUZZIEEE), 2015 IEEE International Conference on. 2015. IEEE, pp 1-8

Papageorgiou, E.I., Salmeron J.L., 2013. A review of fuzzy cognitive maps research during the last decade. IEEE Transactions on Fuzzy Systems. 21, 66-79

Penn, A.S., Knight C.J., Lloyd D.J., Avitabile D., Kok K., Schiller F., Woodward A., Druckman A., Basson L., 2013. Participatory development and analysis of a fuzzy cognitive map of the establishment of a bio-based economy in the Humber region. PloS one. 8, e78319

Peters, A.J., Walter-Shea E.A., Ji L., Vina A., Hayes M., Svoboda M.D., 2002. Drought monitoring with NDVI-based standardized vegetation index. Photogrammetric Engineering and Remote Sensing. 68, 71-75

Pirrone, N., Trombino G., Cinnirella S., Algieri A., Bendoricchio G., Palmeri L., 2005. The Driver-Pressure-State-Impact-Response (DPSIR) approach for integrated catchment-coastal zone management: preliminary application to the Po catchment-Adriatic Sea coastal zone system. Regional Environmental Change. 5, 111-137

Poteete, A., Ostrom E., 2005. Bridging the qualitative-quantitative divide: strategies for building large-N databases based on qualitative research, in: 101st Annual Meeting of the American Political Science Association. 2005. pp 1-4

R.P.P.C. 2014. Rafsanjan Pistachio Producers Cooperative [Online]. Rafsanjan, Iran: Rafsanjan Pistachio Producers Cooperative. Available: http://www.rppc.ir/ [Accessed August 30, 2014].

Rahimi, N., Jetter A.J., Weber C.M., Wild K., 2018. Soft Data analytics with fuzzy cognitive maps: modeling health technology adoption by elderly women, in: Advanced Data Analytics in Health. Springer, pp 59-74

Rahnama, M., Moafi H., 2009. Investigation of land subsidence due to groundwater withdraw in Rafsanjan plain using GIS software. Arabian Journal of Geosciences. 2, 241-246

Razavi, S., 2005. Pistachio production, Iran vs. the world, in: IV International Symposium on Pistachios and Almonds 726. 2005. pp 689-694

Rdpi 2009. Integrated Water Resources Management and Planning in Kerman Basin. Kerman Regional Water Authority Rahbord Danesh Pooya Institute. 
Reckien, D., 2014. Weather extremes and street life in India-Implications of Fuzzy Cognitive Mapping as a new tool for semi-quantitative impact assessment and ranking of adaptation measures. Global. Environ. Change. 26, 1-13. doi:doi:10.1016/j.gloenvcha.2014.03.005

Reckien, D., 2016. Comparison of stakeholder-generated FCMs across generation methods and metrics, in: Fuzzy Systems (FUZZ-IEEE), 2016 IEEE International Conference on. 2016. IEEE, pp 1383-1390

Reckien, D., Wildenberg M., Bachhofer M., 2013. Subjective realities of climate change: how mental maps of impacts deliver socially sensible adaptation options. Sustainability Science. 8, 159-172

Reckien, D., Wildenberg M., Deb K., 2011. Understanding potential climate change impacts and adaptation options in Indian megacities, in: Resilient Cities. Springer, pp 15-34

Robinson, D.T., Brown D.G., Parker D.C., Schreinemachers P., Janssen M.A., Huigen M., Wittmer H., Gotts N., Promburom P., Irwin E., 2007. Comparison of empirical methods for building agent-based models in land use science. J. Land. Use. Sci. 2, 31-55. doi:10.1080/17474230701201349

Roudi-Fahimi, F., 2002. Iran's family planning program: responding to a nation's needs. Population Reference Bureau Washington, DC,

Salehi, M.H., Hosseinifard J., 2012. Soil and groundwater relationships with pistachio yield in the Rafsanjan Area, Iran. Communications in Soil Science and Plant Analysis. 43, 660-671

Salliou, N., Barnaud C., Vialatte A., Monteil C., 2017. A participatory Bayesian Belief Network approach to explore ambiguity among stakeholders about socio-ecological systems. Environmental Modelling \& Software. 96, 199209

Salmeron, J.L., Froelich W., Papageorgiou E.I., 2016. Forecasting daily water demand using fuzzy cognitive maps, in: Time Series Analysis and Forecasting. Springer, pp 329-340

Schelling, T.C., 2006. Micromotives and macrobehavior. WW Norton \& Company,

Schieritz, N., 2002. Integrating system dynamics and agent-based modeling, in: Proceedings of the XX International Conference of the System Dynamics society. 2002. Citeseer,

Schlueter, M., Mcallister R., Arlinghaus R., Bunnefeld N., Eisenack K., Hoelker F., Milner-Gulland E., Müller B., Nicholson E., Quaas M., 2012. New horizons for managing the environment: A review of coupled socialecological systems modeling. Natural Resource Modeling. 25, 219-272

Schlüter, M., Baeza A., Dressler G., Frank K., Groeneveld J., Jager W., Janssen M.A., Mcallister R.R., Müller B., Orach K., 2017. A framework for mapping and comparing behavioural theories in models of socialecological systems. Ecol. Econ. 131, 21-35. doi:10.1016/j.ecolecon.2016.08.008 
Sci 2014. Statistical Centre of Iran. Management and Planning Organization.

Shafiei, E., Stefansson H., Asgeirsson E.I., Davidsdottir B., Raberto M., 2013. Integrated agent-based and system dynamics modelling for simulation of sustainable mobility. Transport Reviews. 33, 44-70

Shakoori, A., 2006. Planning and Agricultural Development in Iran. Critique: Critical Middle Eastern Studies. 15, 265-282

Singh, P.K., Chudasama H., 2017. Pathways for drought resilient livelihoods based on people's perception. Climatic Change. 140, 179-193

Singh, P.K., Nair A., 2014. Livelihood vulnerability assessment to climate variability and change using fuzzy cognitive mapping approach. Climatic Change. 127, 475-491

Solaimani, K., Mortazavi S.M., 2008. Investigation of the land subsidence and its consequences of large groundwater withdrawal in Rafsanjan, Iran. Pakistan Journal of Biological Sciences: PJBS. 11, 265-269

Soler, L.S., Kok K., Camara G., Veldkamp A., 2012. Using fuzzy cognitive maps to describe current system dynamics and develop land cover scenarios: a case study in the Brazilian Amazon. Journal of Land Use Science. 7, 149-175

Stach, W., Kurgan L., Pedrycz W., 2005. A survey of fuzzy cognitive map learning methods. Issues in Soft Computing: Theory and Applications, 71-84

Stave, K., 2010. Participatory system dynamics modeling for sustainable environmental management: Observations from four cases. Sustainability. 2, 2762-2784

Sun, Z., Lorscheid I., Millington J.D., Lauf S., Magliocca N.R., Groeneveld J., Balbi S., Nolzen H., Müller B., Schulze J., 2016. Simple or complicated agent-based models? A complicated issue. Environ. Modell. Softw. 86, 56-67. doi:10.1016/j.envsoft.2016.09.006

Sun, Z., Müller D., 2013. A framework for modeling payments for ecosystem services with agent-based models, Bayesian belief networks and opinion dynamics models. Environ. Modell. Softw. 45, 15-28. doi:10.1016/j.envsoft.2012.06.007

Svarstad, H., Petersen L.K., Rothman D., Siepel H., Wätzold F., 2008. Discursive biases of the environmental research framework DPSIR. Land Use Policy. 25, 116-125

Ten Broeke, G., Van Voorn G., Ligtenberg A., 2016. Which sensitivity analysis method should I use for my agent-based model? J. Artif. Soc. Soc. Simul. 19, 5. doi: $10.18564 /$ jasss. 2857

Thiele, J.C., Kurth W., Grimm V., 2014. Facilitating parameter estimation and sensitivity analysis of agent-based models: A cookbook using NetLogo and R. J. Artif. Soc. Soc. Simul. 17, 11. doi:10.18564/jasss.2503

Unfccc 2010. UNFCCC Glossary of Climate Change Acronyms.

Van Audenhove, L., 2007. Expert interviews and interview techniques for policy analysis. Vrije University, Brussel Retrieved May. 5, 2009 
Van Den Hove, S., 2000. Participatory approaches to environmental policymaking: the European Commission Climate Policy Process as a case study. Ecological Economics. 33, 457-472

Van Vliet, M., Flörke M., Varela-Ortega C., Cakmak E., Khadra R., Esteve P., D'agostino D., Dudu H., Bärlund I., Kok K., 2017. FCMs as a Common Base for Linking Participatory Products and Models, in: Environmental Modeling with Stakeholders. Springer, Cham, pp 145-169

Varela-Ortega, C., Sumpsi J.M., Garrido A., Blanco M.A., Iglesias E., 1998. Water pricing policies, public decision making and farmers' response: implications for water policy. Agricultural Economics. 19, 193-202

Vasslides, J.M., Jensen O.P., 2016. Fuzzy cognitive mapping in support of integrated ecosystem assessments: Developing a shared conceptual model among stakeholders. J. Environ. Manage. 166, 348-356. doi: $10.1016 / j$.jenvman.2015.10.038

Vasslides, J.M., Jensen O.P., 2017. Quantitative vs. Semiquantitative Ecosystem Models: Comparing Alternate Representations of an Estuarine Ecosystem. Journal of Coastal Research. 78, 287-296

Venkatramanan, S., Lewis B., Chen J., Higdon D., Vullikanti A., Marathe M., 2017. Using data-driven agent-based models for forecasting emerging infectious diseases. Epidemics. doi:10.1016/j.epidem.2017.02.010

Virapongse, A., Brooks S., Metcalf E.C., Zedalis M., Gosz J., Kliskey A., Alessa L., 2016. A social-ecological systems approach for environmental management. Journal of Environmental Management. 178, 83-91

Voinov, A., Bousquet F., 2010. Modelling with stakeholders. Environmental Modelling \& Software. 25, 1268-1281

Voinov, A., Jenni K., Gray S., Kolagani N., Glynn P.D., Bommel P., Prell C., Zellner M., Paolisso M., Jordan R., 2018. Tools and methods in participatory modeling: selecting the right tool for the job. Environ. Modell. Softw. 109, 232-255

Walker, B., Carpenter S., Anderies J., Abel N., Cumming G., Janssen M., Lebel L., Norberg J., Peterson G.D., Pritchard R., 2002. Resilience management in social-ecological systems: a working hypothesis for a participatory approach. Conservation Ecology. 6,

Walker, B., Holling C.S., Carpenter S., Kinzig A., 2004. Resilience, adaptability and transformability in social-ecological systems. Ecology and Society. 9,

Water, U., 2007. Coping with water scarcity: challenge of the twenty-first century. 2007 World Water Day,

Wildenberg, M., Bachhofer M., Isak K.G., Skov F., 2014. Use and evaluation of FCM as a tool for long term socio ecological research, in: Fuzzy Cognitive Maps for Applied Sciences and Engineering. Springer, pp 221-236

Wilensky, U. 1999. NetLogo. http://ccl.northwestern.edu/netlogo/. Center for Connected Learning and Computer-Based Modeling: Northwestern University, Evanston, IL. 
Xiang, W.-N., 2013. Working with wicked problems in socio-ecological systems: Awareness, acceptance, and adaptation. Landscape and Urban Planning, 1-4

Yang, L., Gilbert N., 2008. Getting away from numbers: Using qualitative observation for agent-based modeling. Advances in Complex Systems. $11,175-185$

Zimmerman, L., Lounsbury D.W., Rosen C.S., Kimerling R., Trafton J.A., Lindley S.E., 2016. Participatory system dynamics modeling: Increasing stakeholder engagement and precision to improve implementation planning in systems. Administration and Policy in Mental Health and Mental Health Services Research. 43, 834-849 
Appendix 


\section{Appendix 1. Individual or group interviews}

Both methods of individual and group interview were tested in preliminary phase of the field work to explore the pros, cons and feasibility of each in our case. Two group interview sessions were conducted per each stakeholder group. Two P\&R groups of 4 and 5 members and two farmer groups of 9 and 11 were interviewed collectively. Based on the exploratory interviews, the group interview among academician and policy makers were more successful than the farmers since they were almost vocally and forcefully equal. Therefore, the synergy among participant could help them to develop the model upon each other's knowledge. But in farmers group interview this could not easily happen since the local farmers and less educated ones had little chance to voice their views in front of the more well-knowledge peers. The overwhelming personalities of some farmers in a group discussion is inevitable due to their hierarchical position in the farming community. However, due to the high sensitivity of water crisis in Iran and its political aspect involved, participants from government could not freely share their critical views and insights in a group interview. Moreover, it was very difficult to arrange the group session with policy makers and researchers, since they were either busy or from separated organization and companies and even from different cities. Therefore, finally we conducted whole the interviews individually to elicit the most diverse knowledge for equitable multi-person multi-objective decision making (Gray et al., 2014).

\section{Appendix 2. Stakeholders sample size}

Since FCM is a qualitative data collection method, its knowledge reliability increases with expert sample size (Kosko, 1986b). To determine the sufficient sample size the Monte Carlo technique is suggested by Özesmi and Özesmi (2004) and deployed by Singh and Nair (2014) in FCM. The accumulation carve in this technique represents the number of new variables added per map versus the total number of maps. Where the curve stabilises indicates the minimum maps required to sample the population sufficiently. The P\&R accumulation curve stabilised after 28 maps and we continued for about 10 more maps to make sure about the stabilisation. Farmers curve stabilised at 33 but we still continued after that since our farmer samples were from four different districts of township to get equal understanding from whole region. Eventually a total number of 100 participants were interviewed out of which 60 participants were farmers and 40 were policymakers and researchers. Since a large number of farmers and land owners in Rafsanjan are living out of the region, the interviews were done over August and September 2015 when most of the expat land owners come back to their fields for production harvesting. 


\section{Appendix 3. DPSIR framework}

The major environmental problem in this case study is related to water situation change. Therefore, among many DPSIR frameworks, we defined the categories of our DPSIR conceptual model similar to the categories that water management studies used in their frameworks. E.g. (Borja et al., 2006, Pirrone et al., 2005). This analytical framework is developed base on spatial data-sets (Mehryar et al., 2015) and previous studies and reports. The elements of DPSIR framework in our case are presented in (Figure 3.2):

Drivers are underlying socio-economic developments in societies and environmental system resulting into the major change in the system which enhances consumption of water as well as weak water governance. The drivers that have been considered in case of Rafsanjan are population growth, weak government control and monitoring, economic development plans, climate change, and subsidies and free water.

Pressures are the direct or indirect form of stresses caused by driving forces. They effect a change in the normal flow or quality of GW and they pose higher risks on State of GW if not checked. The pressures in Rafsanjan are considered as agriculture area expansion, unregulated groundwater use, excessive wells construction, water consumption pattern and subsidy.

State is the situations or trends of water resources which are not considered to be normal or healthy. It refers to measurement indicators like quantity or quality of the water resources. Like GW pollution, GW level in our case.

Impact is the consequence of changes in state of the water situation which is the effect of the pressure by nature. Impacts may be either negative or positive on both environment and socio-economic systems. It might be direct or indirect through the other impact factors. Like ecosystem modification (land cover change and land subsidence) and socio-economic pressure (job change, unemployment, regional and individual economy change, agriculture production decrease, immigration, social conflict and decline in sustainable investment) in this case.

Response is the human reaction to environmental changes. This is the most controversial category. Like GW and energy pricing, wells control, water supply projects, agriculture area reduction by people or government.

The most dominant proposed strategies (response) to combat water scarcity in the region are divided to four types: 1 ) the supply providing strategies like big water transfer project, 2) monitoring strategies, like installing wells counter and police supervision, 3) limitation strategies like water and energy pricing 
and agriculture land reduction; 4) change of irrigation or agriculture systems like using drip irrigation

\section{Appendix 4. FCM application}

\subsection{Condensation}

In our case study, the first augmented FCM of farmers, combined of 60 individual maps, contained 134 concepts and 321 connections which has been reduced to 33 concepts and 124 connections after condensation. The first augmented P\&R FCM had also 152 concepts and 363 connections which have been reduced to 34 concepts and 209 connections after condensation.

The concepts deleted by quantitative condensation:

\begin{tabular}{|l|l|}
\multicolumn{1}{l}{ Table 4. The concepts deleted by quantitative condensation } \\
\hline Farmers & $\begin{array}{l}\text { Urban\&Rural expansion/ environmental pollution/health } \\
\text { problem/ industrial water pollution/ crop modification/ } \\
\text { water purchase }\end{array}$ \\
\hline $\begin{array}{l}\text { Policy makers } \\
\text { and Researchers }\end{array}$ & $\begin{array}{l}\text { Villeinage/ pest outbreak/ water marketing/ water } \\
\text { rationing }\end{array}$ \\
\hline
\end{tabular}

\subsection{Weighting the connections}

The potential practical mistake was the tendency of respondents to give the changing value of each concept instead of the influence weight of their connection with other concepts. For example, when they were asked about the influence of "water shortage" on "agriculture production" they tended to assign the decreasing percentage of their pistachio production in last years as the connection value. So it was needed to be clearly asked that "How much the increase or decrease of $X$ has caused the increase or decrease of $Y$ ?" Moreover, it was constantly explained to the respondents that the connection weight is strongly related to the other weights of the system not only the specific relation of two concepts (Jetter and Kok, 2014).

\section{Appendix 5. FCM structure analysis}

To describe the analysis result of the model first we explain briefly the FCM analysis method. The FCMs' properties are analyzed by using graph theories. The graph theory of structure analysis focuses on network characteristics like number of variables, number of connections and the related indices. These indices include in-degree, out-degree, centrality, density and complexity. Indegree and out-degree of each variable indicate the sum of all connections' values entering and existing that variable respectively. In the matrix, in-degree is the column sum of a variable values and out-degree is their row sum. Centrality $(C)$ is the summation of the in-degree and out-degree of a variable. Density (D) of a cognitive map, indicating how connected the maps are, is 
calculated by dividing the number of connections in the map to the number of all its possible connections. So it is represented by $D=C / N 2$, where $C$ is the number of connections and $\mathrm{N}$ is the number of variables or nodes.

Using these indices, all components of FCMs are categorized in three ways: transmitter, receiver, and ordinary. Transmitter variables have positive outdegree and zero in-degree, whereas Receiver components have positive indegree and zero out-degree. Ordinary variables have both non-zero in-degree and out-degree. Comparing the total number of receiver and transmitter in a map gives us an insight about the complexity or hierarchy of the system (Özesmi and Özesmi, 2004). A larger number of receiver variables indicate the higher degree of complexity since they consider many outcome as the result of the system. Whereas, the large number of transmitter indicates the higher degree of formal hierarchical system since they represent more forcing function and more consequent top-down way of thinking (Özesmi and Özesmi, 2004, Gray et al., 2012). 
Appendix 6. Typology of perceptible and measurable variables in our case study

Concepts

National economic

National economic development Mono-economy Climate change Population growth Small holding Lack of peoples' participation Traditional agriculture/irrigation Lack of government control

Lack of land document Agriculture area expansion Groundwater exploitation Number of illegal wells Number of legal wells Subsidy

GW shortage

Water quality

Agriculture production

Farmers vulnerability Land subsidence Orchards dry up and LCC

Regional economy

Sustainable investing

Deepening and transferring wells Desalination Integrated land owning Irrigation area reduction Irrigation system modification Pistachio land transfer Wells turn off

\begin{tabular}{|c|c|c|c|c|}
\hline $\begin{array}{l}\text { Inherently } \\
\text { subjective } \\
\text { (perceptible- } \\
\text { variable) }\end{array}$ & $\begin{array}{l}\text { No-data } \\
\text { (perceptible- } \\
\text { variable) }\end{array}$ & $\begin{array}{l}\text { Better } \\
\text { perceived } \\
\text { (perceptible- } \\
\text { variable) }\end{array}$ & $\begin{array}{l}\text { Inherently } \\
\text { objective } \\
\text { (measurable- } \\
\text { variable) }\end{array}$ & $\begin{array}{l}\text { Hidden } \\
\text { variables } \\
\text { (measurable- } \\
\text { variable) }\end{array}$ \\
\hline & $X$ & & & \\
\hline & & $X$ & & \\
\hline & & & $X$ & \\
\hline & & & $X$ & \\
\hline & & $X$ & & \\
\hline \multicolumn{5}{|l|}{$X$} \\
\hline & & $X$ & & \\
\hline & $X$ & $X$ & & \\
\hline & $X$ & & & \\
\hline & & & $X$ & \\
\hline & & & $X$ & \\
\hline & $X$ & $X$ & & \\
\hline & & & $X$ & \\
\hline & $X$ & $X$ & & \\
\hline & & & $X$ & $X$ \\
\hline & & & $X$ & \\
\hline & & & $X$ & \\
\hline & & $X$ & & \\
\hline & & & $X$ & $X$ \\
\hline & $X$ & $X$ & & \\
\hline & & $X$ & & \\
\hline \multicolumn{5}{|l|}{$X$} \\
\hline & $X$ & $X$ & & \\
\hline & $X$ & $X$ & & \\
\hline & $X$ & $X$ & & \\
\hline & & $X$ & & \\
\hline & & $X$ & & \\
\hline & $X$ & $X$ & & \\
\hline & & $X$ & & \\
\hline
\end{tabular}




\section{Available time-series data and their sources}

Table 1. Population time-series of Rafsanjan Township. Source: Iran national census, https://www.amar.org.ir/english/Iran-at-a-glance/Kerman

\begin{tabular}{|c|c|c|c|c|c|c|c|}
\hline & $\mathbf{1 9 6 6}$ & $\mathbf{1 9 8 6}$ & $\mathbf{1 9 9 6}$ & $\mathbf{2 0 0 6}$ & $\mathbf{2 0 1 1}$ & $\mathbf{2 0 1 6}$ & $\mathbf{2 0 2 1}$ \\
\hline $\begin{array}{c}\text { Current } \\
\text { Population }\end{array}$ & 120,116 & 178,917 & 240,211 & 295,175 & 287,921 & & \\
\hline & & & & & & & \\
\hline $\begin{array}{c}\text { Predicted } \\
\text { Population }\end{array}$ & & 74,039 & 117,531 & 175,372 & 171,197 & & \\
\hline $\begin{array}{c}\text { Urban } \\
\text { population }\end{array}$ & 104,868 & 122,680 & 119,803 & 115,114 & & \\
\hline $\begin{array}{c}\text { Rural } \\
\text { population }\end{array}$ & & 1034,963 & \\
\hline
\end{tabular}

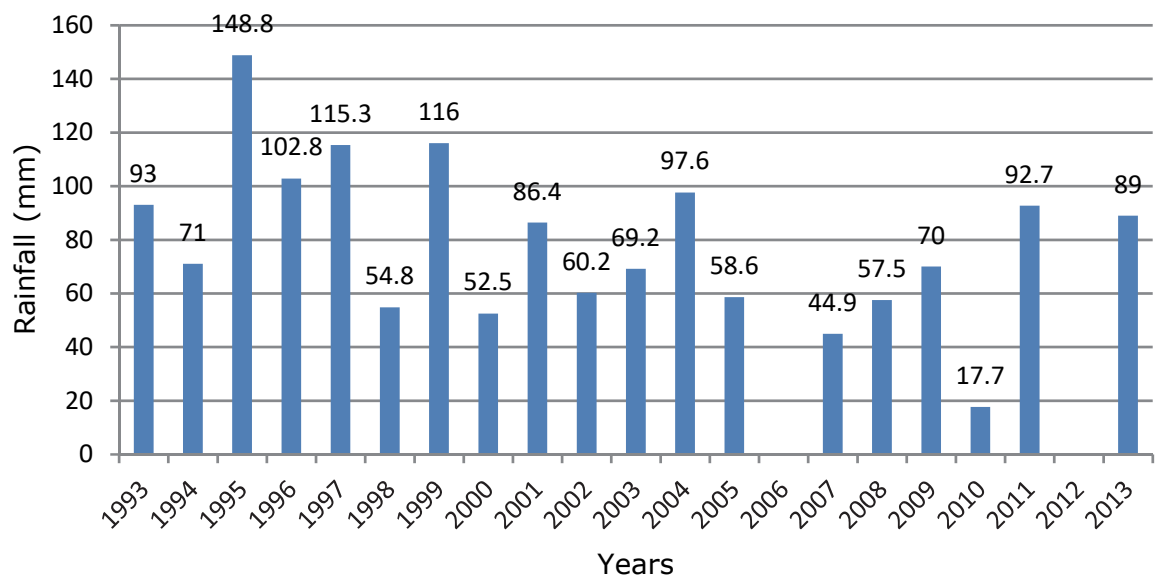

Figure 7. Precipitation time-series of Rafsanjan. Source: Iran Meteorological Organization (IRIMO)

Table 2. Pistachio land area time-series in Rafsanjan. Source: Mehryar et al. (2015) $1986 \quad 86-98 \quad 1998 \quad 98-$

20062006

2014

$\% \quad 2006 \quad 2014$

$\%$

\begin{tabular}{c|ccccccc}
$\begin{array}{c}\text { Pistachio } \\
\text { planted } \\
\text { area (ha) }\end{array}$ & 73,000 & 17 & 86,000 & 20 & 104,000 & 3 & 108,000
\end{tabular}




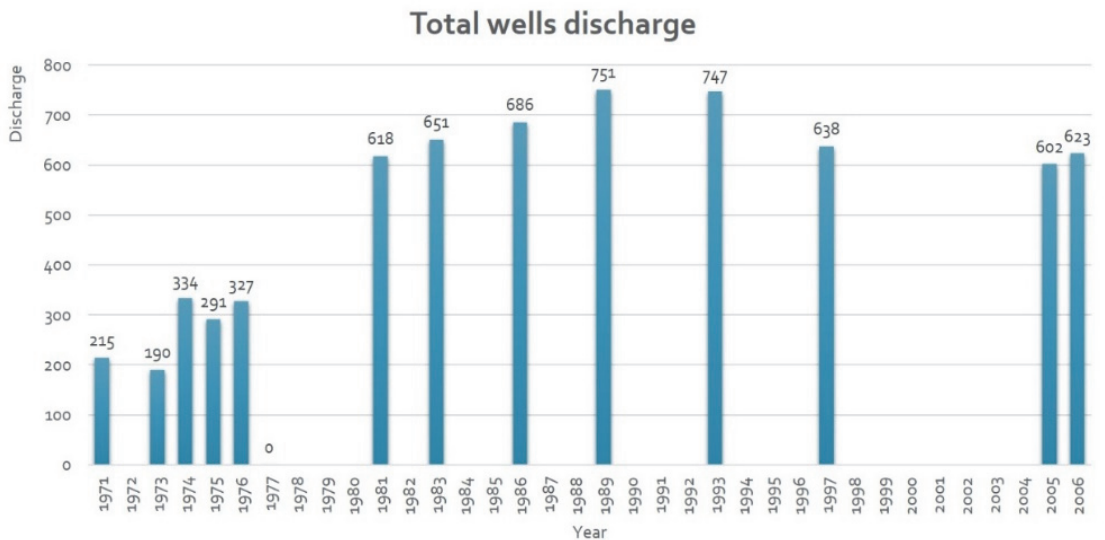

Figure 8a. Total wells discharge time-series in Rafsanjan. Source: Official reports

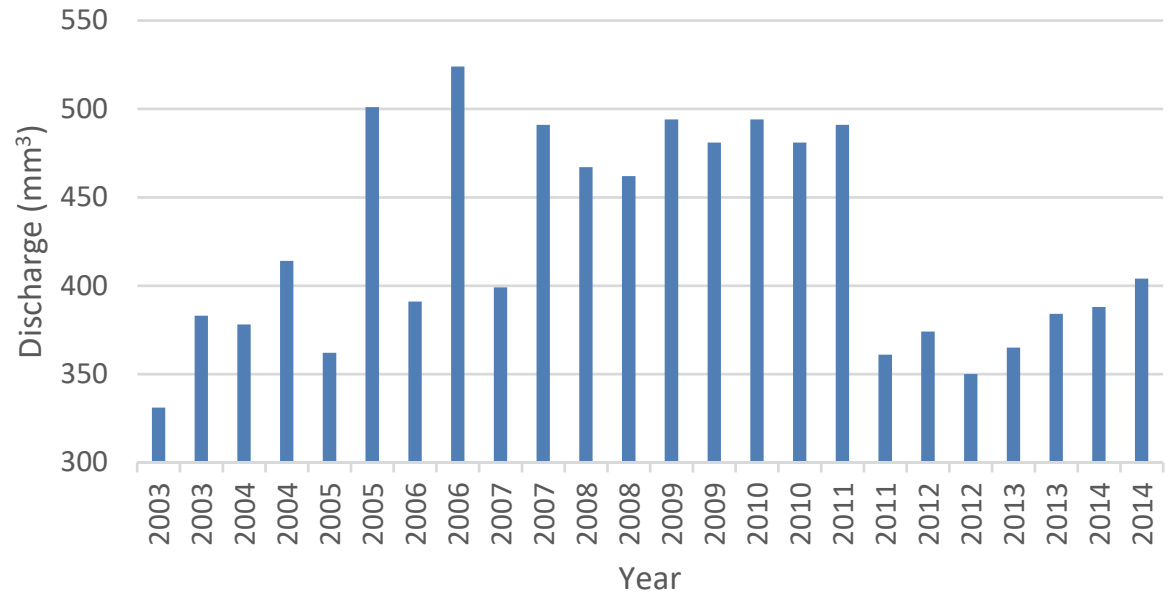

Figure 9b. Total wells discharge time-series in Rafsanjan. Source: Official reports 


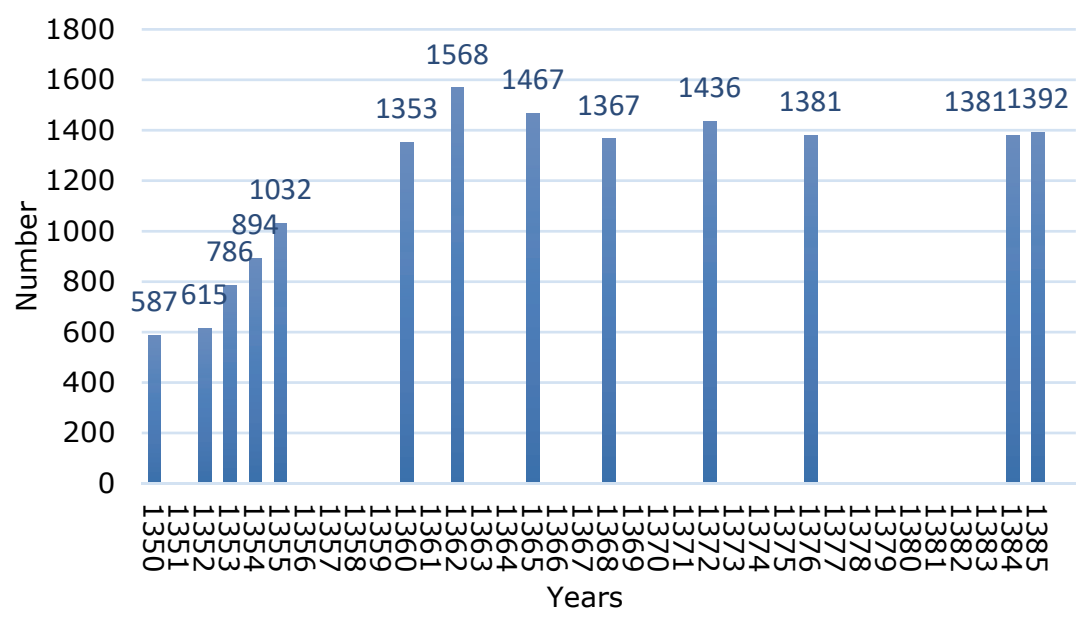

Figure 10. Total number of wells time-series in Rafsanjan. Source: Official reports

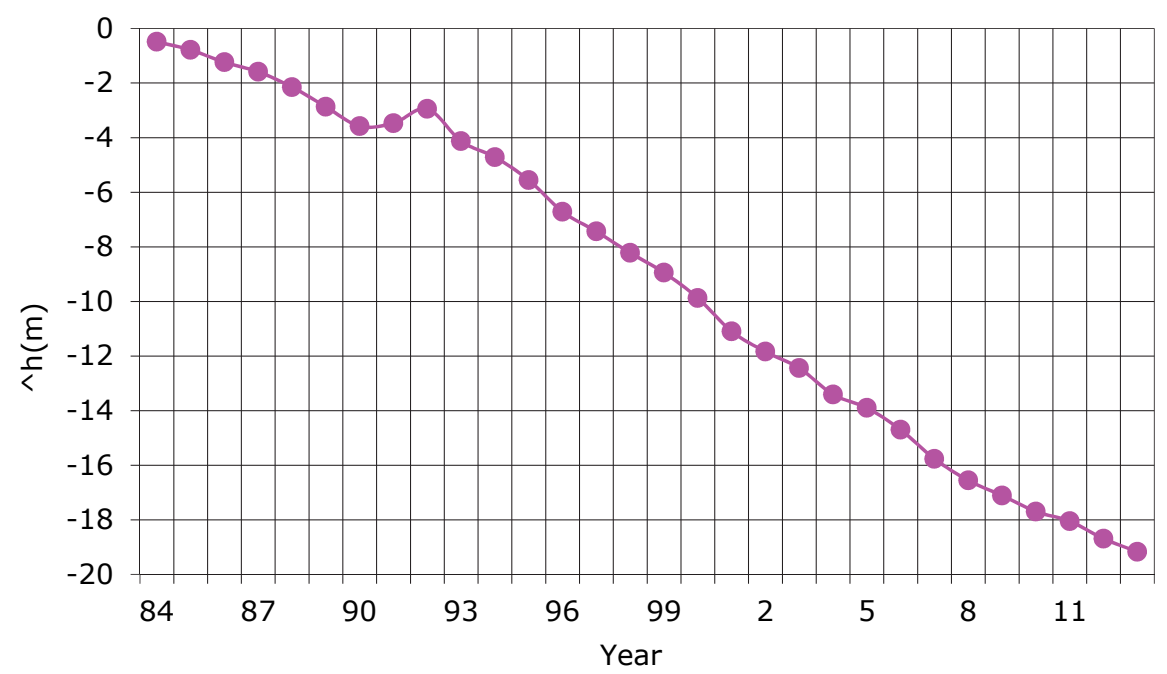

Figure 11. Groundwater level time-series in Rafsanjan. Source: http://wrbs.wrm.ir/ 


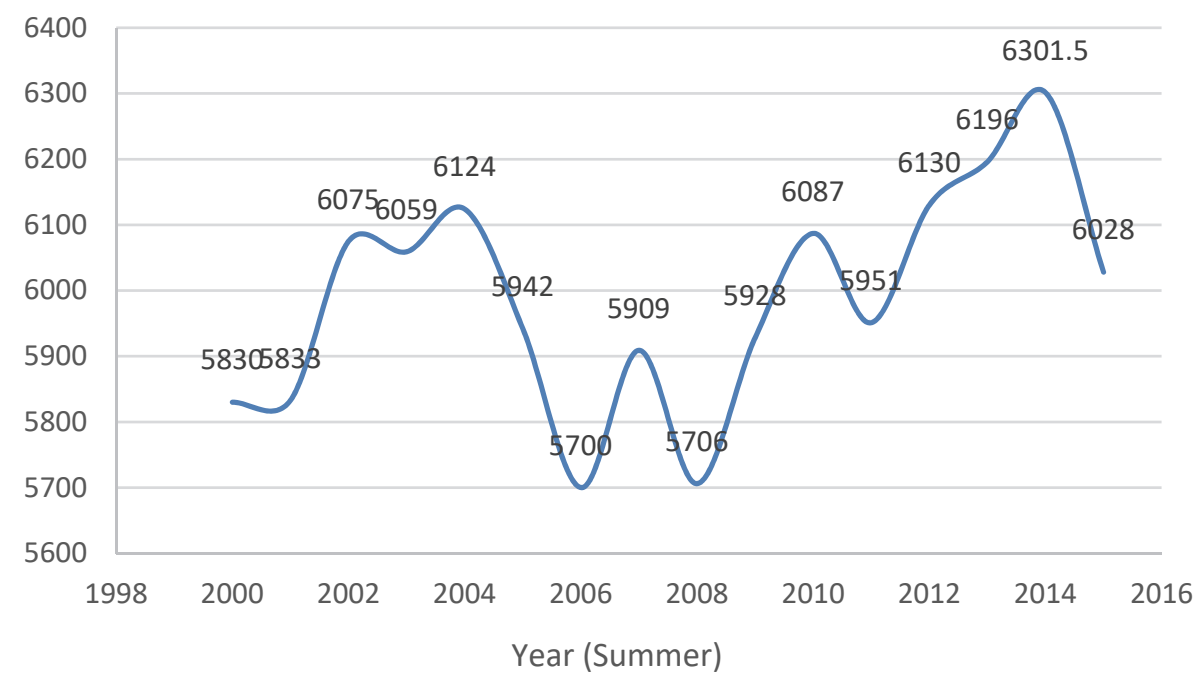

Figure 12. Groundwater quality (average) time-series in Rafsanjan. Source: http://wrbs.wrm.ir/

Table 3. Pistachio production, export and efficiency time-series in Rafsanjan. Source: iranpistachio.org/fa/sample/before

\begin{tabular}{|l|l|l|l|}
\hline & Production (Ton) & Efficiency (ton/ha) & Export \\
\hline 1982 & & & 1698 \\
\hline 1983 & 19677 & & 7180 \\
\hline 1984 & 6669 & & 3492 \\
\hline 1985 & 15583 & & 4374 \\
\hline 1986 & 35775 & 0.49006849 & 9335 \\
\hline 1987 & 18297 & 0.24697974 & 20079 \\
\hline 1988 & 40137 & 0.53397813 & 17917 \\
\hline 1989 & 23300 & 0.30557778 & 24708 \\
\hline 1990 & 44526 & 0.57577717 & 34532 \\
\hline 1991 & 59932 & 0.76429255 & 58057 \\
\hline 1992 & 60479 & 0.76076128 & 54505 \\
\hline 1993 & 47018 & 0.58348742 & 56141 \\
\hline 1994 & 34370 & 0.42087089 & 46560 \\
\hline 1995 & 73370 & 0.88667867 & 55370 \\
\hline 1996 & 54534 & 0.65053084 & 63631 \\
\hline 1997 & 22080 & 0.26003086 & 18440 \\
\hline 1998 & 519709 & 0.60440698 & 32457 \\
\hline 1999 & 33062 & 0.37464023 & 30107 \\
\hline 2000 & 48000 & 0.53038674 & 39099 \\
\hline 2001 & 35000 & 0.37735849 & 51244 \\
\hline 2002 & 6000 & 0.06315789 & 39662 \\
\hline 2003 & 27719 & 0.28502828 & 47346 \\
\hline 2010 & 40000 & 0.37735849 & \\
\hline 2011 & 40000 & 0.37558685 & \\
\hline 2012 & 41000 & 0.38317757 & \\
\hline 2013 & 38000 & 0.35348837 & \\
\hline 2014 & 80000 & 0.74074074 & \\
\hline 2015 & 49000 & & \\
\hline & & & \\
\hline & & & \\
\hline
\end{tabular}




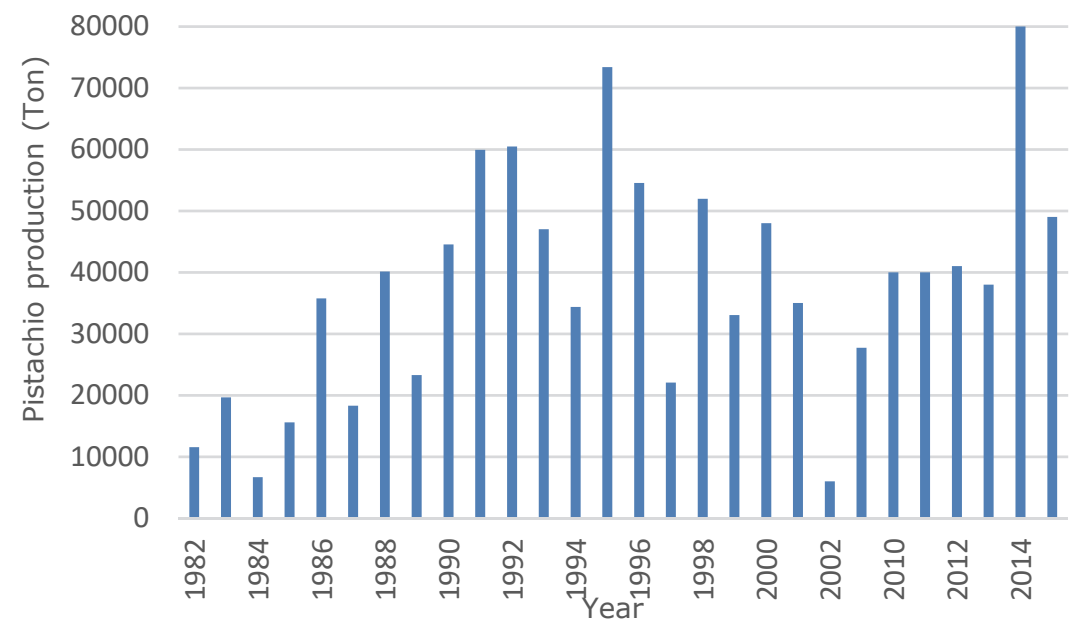

Figure 13. Pistachio production time-series in Rafsanjan. Source: iranpistachio.org/fa/sample/before

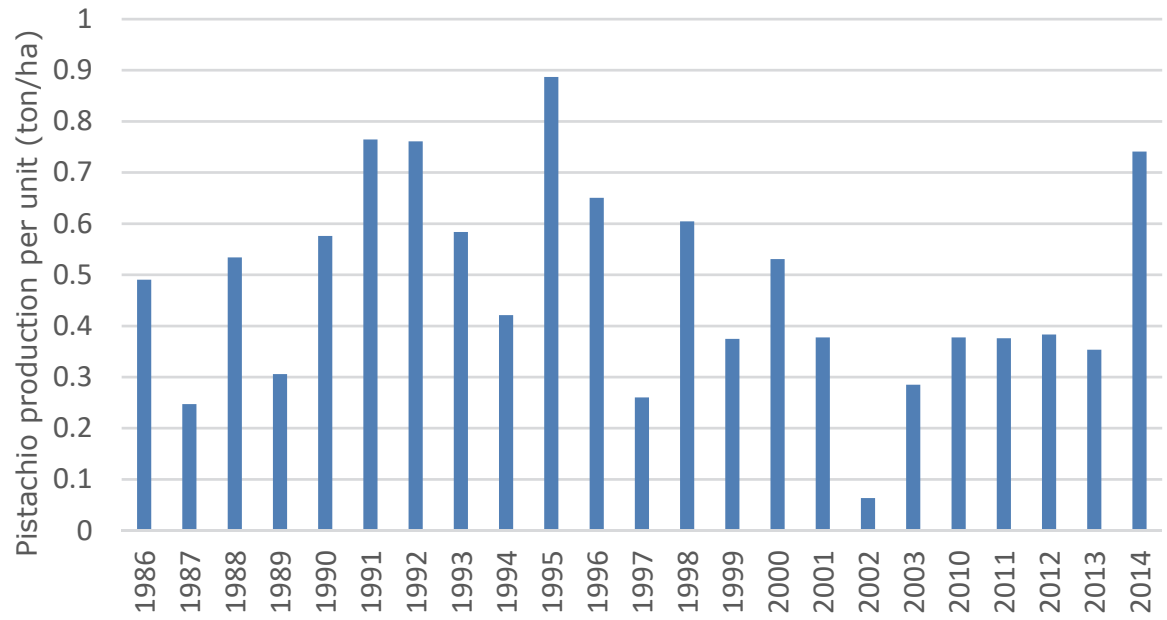

Year

Figure 14. Pistachio production efficiency time-series in Rafsanjan. Source: iranpistachio.org/fa/sample/before

\section{Appendix 7. Comparing D-FCM and FP-FCM}

More important results of connection value differences between D-FCM and FP$\mathrm{CM}$ are as follows:

1) Farmers perceived a very strong relationship between climate change and groundwater quality, whereas the measured-data and policy makers' model showed a very weak relationship among these two. 
2) The impact of pistachio land expansion on groundwater level decline was very strong and on ground water quality was weak according to the D-FCM and PP-FCM, while farmers perceived the increase of pistachio lands to have had a very strong impact on groundwater quality and medium impact on groundwater level.

3) The increase of groundwater exploitation has had a strong impact on the number of legal wells according to the measured-data and policy makers' perception, but farmers have not mentioned any relation between these two concepts. This is because farmers mostly believed the increase of illegal wells to be more important and thus attributed higher weights to the illegal wells rather than legal ones. Nevertheless measured-data indicated that legal wells were also highly influenced by the increase of groundwater exploitation not only the illegal wells. It also had largely resulted to the increase of groundwater exploitation. This is because of the over-exploitation of legal wells which had more negative impact than growing number of illegal wells.

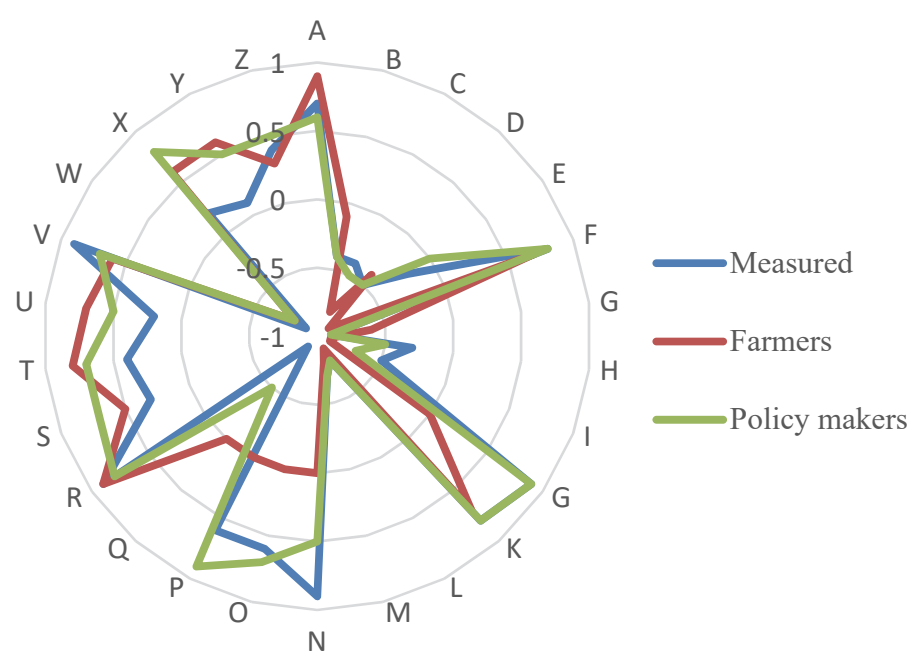

Figure 1. Values of connections in three different FCMs: D-FCM, PP-FCM, FP-FCM.

\section{Appendix 8. Overview, Design concepts and Details}

\section{Overview}

\subsection{Purpose}

This model simulates different farmers' decisions and actions to adapt to the water scarce situation in Rafsanjan, Iran. This simulation helps to investigate how stakeholders' strategies may impact on macro-behavior of the system i.e. overall groundwater use change and emigration of farmers.

\subsection{Entities, state variables, and scales}


Agents: In this model, agents represent the total number of 154 farmers in three types of 21 large, 49 medium, and 84 small farmers. Their attributes are 1) their land size: 250 ha $>$ large farms $>80$ ha $>$ medium farms $>15$ ha $>$ small farms, 2) their sub-region, and 3) the actions they take.

Environment: Farmers are distributed across a stylized representation of the Rafsanjan landscape. As Rafsanjan is spatially heterogeneous, we distinguish nine sub-regions in the ABM, out of which two are representing non-vegetated area. Each sub-region consists of 15 by 15 cells, leading to a total of $45 * 45$ cells. Each cell can have one farmer owning the cell; each farmer may have 1 or more cells. Agents are distributed equally across the seven regions and randomly within each region. Each cell represents 5 ha of pistachio land. Cells are characterized by 1 . Depth of groundwater level 2. Groundwater quality, 3. Productivity, 4. Land subsidence level, 5. Groundwater use 6. Well's depth, and 7. Allowed well's depth.

Temporal resolution: Time step is 1 month, and variables' changes are monthly or yearly. The temporal extend of the model is 15 years, i.e. 180 time steps.

\subsection{Process overview and scheduling}

Basically this model considers two main process in each time step:

1) Cells' update: There are two types of updates for cells' properties, 1) based on variables' dynamic changes collected from empirical data, e.g. groundwater level change and land subsidence level change, 2) based on impacts of actions -from previous step- on environment variables.

2) Agents' decision-making: First, all agents check their groundwater access. If an agent is not satisfied with the groundwater access, it enters the decision making process to adapt its groundwater access. Otherwise, it exits this time step.

\section{Design concepts}

\subsection{Basic principles}

The model is informed by Fuzzy Cognitive Mapping (FCM) models developed from time-series data (where formal data is available) and stakeholders' perception via interviews and mind mapping (for variables without formal data). From FCM models, we learn how macro level variables of a system, i.e. groundwater, regional economy, production, land use change, water management, human interventions etc., are influencing each other. Therefore, we know what are the causes and effects of different possible adaptive actions from farmers. Causes are the conditions of each action and effects are the impacts of each action on properties of agents or environment in the ABM. From FCM, we also have the weight or level of impacts of each action on other variables. Notice that in FCM we have the level of causal relations, but not the 
absolute value of each variables. Therefore, this models is meant to compare the impact of different adaptive strategies on specific variables rather than calculate or forecast the absolute value of each variables.

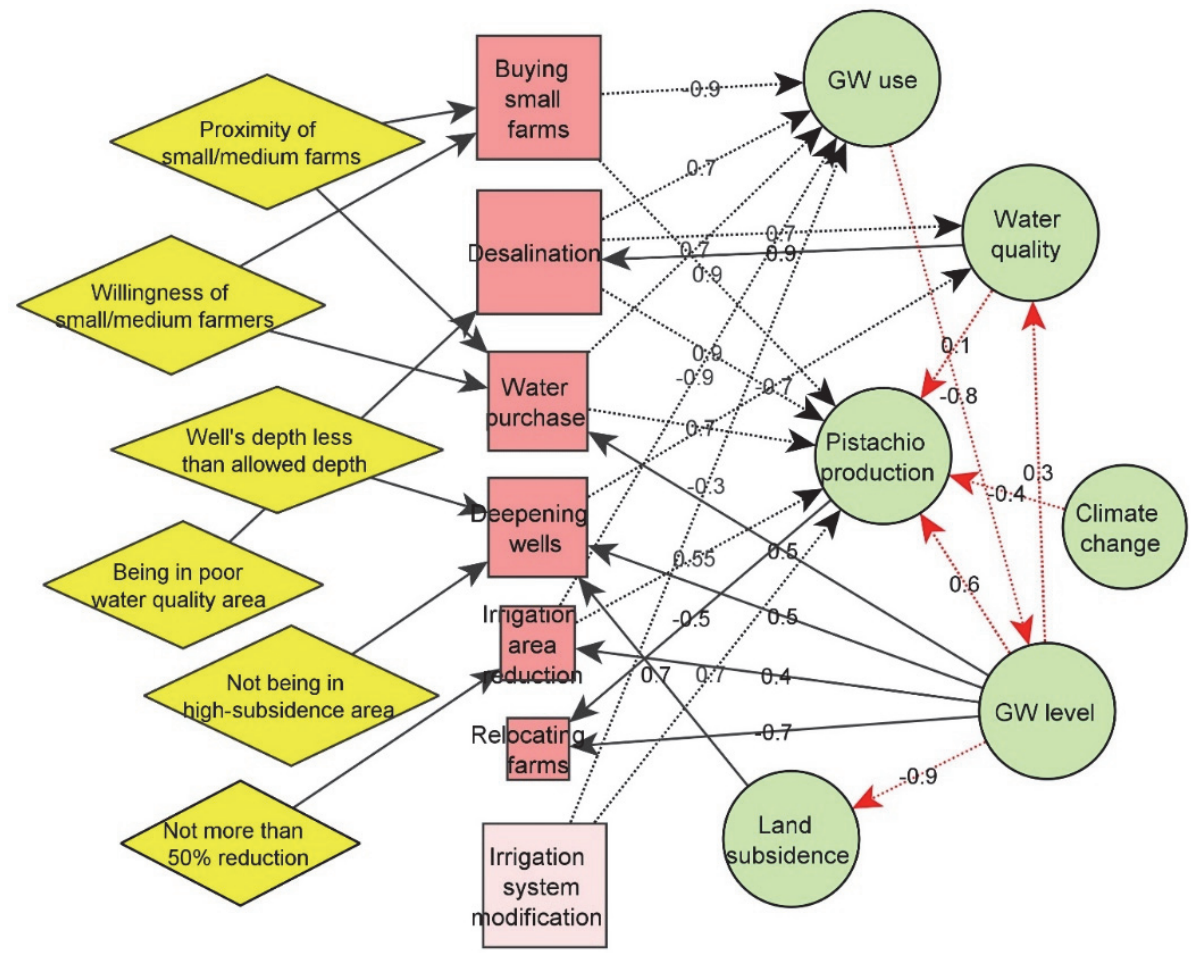

Figure 1. Large-farmers' FCM combined with objective data. The red squares show farmers' actions and their size shows the number of farmers who took this action i.e. level of preference or priority of actions. Nodes with input to (yellow diamonds) and output from (green circles) actions represent conditions and impacts of those actions, respectively. Black and red lines represent perceived connections and data-driven connections, respectively. Solid and dashed lines show positive and negative causal connections, respectively. 


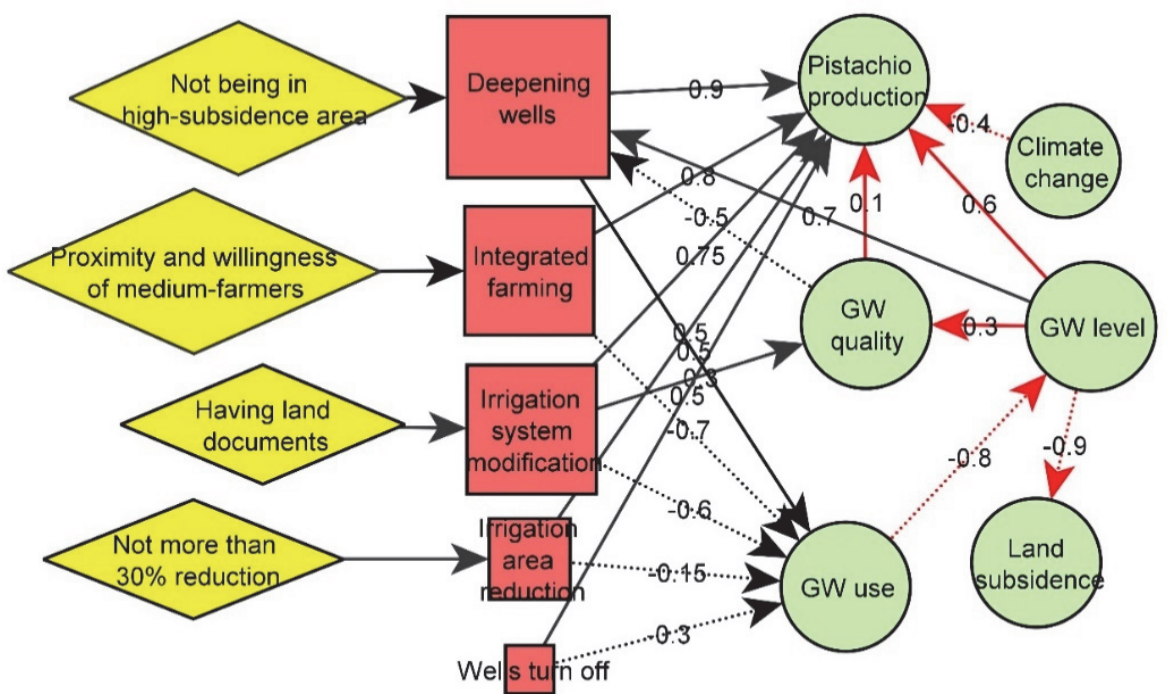

Figure 2. Medium farmers' FCM combined with objective data

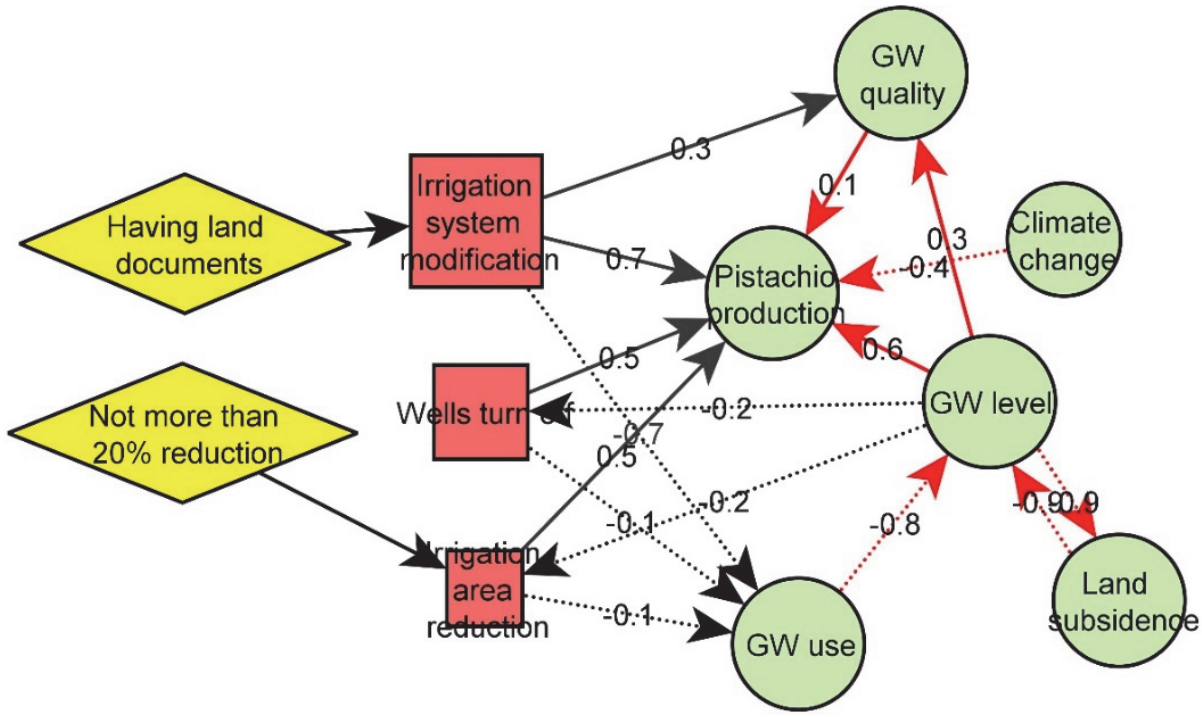

Figure 3. Small-farmers' FCM combined with Objective data

\subsection{Emergence}

This model is designed to explore the relationship between farmers' adaptive actions towards water scarcity and two related emergent phenomena: overall groundwater use change and emigration of farmers. Overall groundwater use results from aggregated individual farmers' water use that may change over time due to their dynamic adaptive actions towards water scarcity and 
interactions with other farmers. Emigration of farmers results from their both groundwater access and interactions with other farmers.

\subsection{Adaptation}

All individual farmers do these adaptive actions to increase their ground water access or control their water use, and eventually increase their farms' production.

\subsection{Objectives}

Farmers want to keep their pistachio production and keep their access to groundwater for irrigation. If they are unsatisfied they leave and relocate their farms to the other regions.

\subsection{Learning and Prediction}

Individuals do not learn from their own experiences, i.e. positive or negative impacts of previous actions. Also, individuals do not predict or estimate future consequences of their decisions.

\subsection{Sensing}

Farmers have the full knowledge about the state of their groundwater access, their available options, their neighbors' willing to sell/buy land/water or integrate their lands, and their lands' groundwater quality, land subsidence, and allowed wells' depth. They do not have knowledge about the state of the overall groundwater level change in their region-which can help them in predicting future groundwater situation.

\subsection{Interaction}

Large farmers buy land and water from small and medium farmers. Medium farmers share their farms for efficient irrigation and farming. The structure of their social network for selling and buying land and water is emergent during the simulation. When the vulnerability of small or medium farmers becomes high, they will be willing to sell off their water and lands to the large farmers.

\subsection{Stochasticity}

Randomness is used in two processes: 1 ) executing of actions with the same priority, e.g. action number $3 \& 4$ in priority 2 of large farmers' actions list, and 2 ) the initial distribution of agents (farmers), farm sizes and initial values of parameters.

\subsection{Collectives}

There are no collectiveness among agents.

\section{Details:}

The model is implemented in NetLogo 6.0.1 (Wilensky, 1999) and available at

\subsection{Initialization and input data}

Initial patch properties (i.e. groundwater use, groundwater quality, land subsidence, ground water level, and well's depth) are extracted from GIS attribute data of 1369 wells in Rafsanjan collected in 2015 by Iran Water 
Resource Management Company (http://wrbs.wrm.ir/). In each sub-region an interval of initial values are calculated as explained below and them randomly distributed over the patches.

Initial groundwater use: groundwater use per hectare per month is calculated by:

$$
\text { Groundwater use }\left(\frac{\mathrm{mm}^{3}}{\mathrm{ha.mo}}\right)=\frac{\text { Discharge } \times 360 \times \mathrm{H} \times 30}{\mathrm{ha} \times 10^{6}}
$$

Discharge (of wells' pumps) $=$ volume of extracted water per second $\left(\mathrm{m}^{3} / \mathrm{s}\right.$ ) for each well

$\mathrm{H}=$ Number of hours per day with wells' pump on (taken from GIS data for each well)

$\mathrm{Ha}=$ Pistachio land area covered with each well (taken from GIS data)

Initial wells' depth, groundwater level are calculated by their mean \pm standard deviation

Initial land subsidence and groundwater quality are distributed in five levels of very low, low, medium, high and very high

\begin{tabular}{|l|l|l|}
\hline 1 & & 7 \\
\hline 2 & & 6 \\
\hline 3 & 4 & 5 \\
\hline
\end{tabular}

Figure 15. Spatial representation of 7 regions

Table 1. Initial values of environment parameters.

\begin{tabular}{|c|c|c|c|c|c|c|c|}
\hline & $\begin{array}{l}\text { Locatio } \\
\text { n } 1\end{array}$ & $\begin{array}{l}\text { Locatio } \\
\text { n } 2\end{array}$ & $\begin{array}{l}\text { Location } \\
3\end{array}$ & $\begin{array}{l}\text { Location } \\
4\end{array}$ & $\begin{array}{l}\text { Locatio } \\
\text { n } 5\end{array}$ & $\begin{array}{l}\text { Locatio } \\
\text { n } 6\end{array}$ & $\begin{array}{l}\text { Location } \\
7\end{array}$ \\
\hline $\begin{array}{l}\text { GW use } \\
\left(m^{3} / h a\right)\end{array}$ & 120 & 121 & 122 & 122 & 122 & 124 & 124 \\
\hline $\begin{array}{l}\text { Well's } \\
\text { depth (m) }\end{array}$ & $95-100$ & $\begin{array}{c}105- \\
110\end{array}$ & $130-140$ & $130-140$ & $135-145$ & $140-150$ & $125-135$ \\
\hline $\begin{array}{l}\text { GW level } \\
\text { (m) }\end{array}$ & 90 & 100 & 120 & 115 & 120 & 120 & 110 \\
\hline $\begin{array}{l}\text { GW } \\
\text { quality }\end{array}$ & Very low & Very low & high & Very high & $\begin{array}{l}\text { Very } \\
\text { high }\end{array}$ & medium & medium \\
\hline $\begin{array}{l}\text { Land } \\
\text { subsidenc } \\
\text { e }\end{array}$ & Low & low & Very high & Low & high & $\begin{array}{l}\text { High- } \\
\text { Very } \\
\text { high }\end{array}$ & Very high \\
\hline
\end{tabular}




\subsection{Sub models}

Step 1) Update patches:

Indirect impacts of actions from previous time step are calculated at the beginning of the next step as follow:

Indirect impacts of actions are the impacts of variables affected by actions on other variables in FCM. To implement the impact of Variable $A$ onto the Variable $B$ (represented as $A \stackrel{w}{\rightarrow} B$ ) the value of Variable $B$ in the new time step is calculated as:

Step 2) Agents' decision making:

$$
B_{t+1}=B_{t}+B_{t} \times \frac{A_{t}-A_{t-1}}{A_{t-1}} \times w
$$

2.1) each agent checks its groundwater access, based on:

$$
\mathrm{GW} \text { access = depth of GW - Well's depth }
$$

If the agent is not satisfied it continues its decision making, otherwise it ends this time step.

The available actions that agents can take from are as follows:

- Buying small/medium farms: Buying farms from medium or small farmers who are not willing to continue pistachio production in their farms

- Desalination: set up desalination system on farms with poor water quality to remove salt and mineral from saline groundwater.

- Water purchase: Buying water from other farmers

- Deepening wells: Digging water wells to get access to groundwater

- Irrigation area reduction: shrinking (dry-off) small part of the farm to increase efficiency of water use for rest of the farm.

- Integrating farms: integrate irrigation system of some farms together to increase their efficiency.

- Irrigation system modification: changing traditional flood irrigation to drip irrigation.

- Well's turn-off: Increasing the wells' off-time over nights or winters

- Relocating farms: leave the region and buy farm in other areas with better water situation

2.2) Checks conditions of available actions: In this step, each agent check the actions' conditions through their priority order of actions. If the conditions are confirmed, it executes the action and if not it goes to the next action.

2.3) Action execution: Each agent execute possible actions. These executions depends on type of actions.

2.4) Implement impact of actions: execution of each action has specific level of impacts on other variables of the environment. This level of impact 
comes from the FCM model. Therefore, the state of influenced variables (affected variables in FCM) gets updated after execution of each action. Here we only implement the direct impact of actions on other variables. To implement the direct impact of actions $X$ onto variables $A$ of the FCM model (represented as $X \stackrel{w}{\rightarrow} A$ ), in each time step that action $X$ is executed the value of Variable $A$ in that time step is calculated as:

$$
A_{t+1}=A_{t}+\left(A_{t} \times w\right)
$$

For example, when we have desalination $\stackrel{0.7}{\rightarrow}$ groundwater use (in figure 2), whenever that action desalination is executed, it impacts groundwater use by 0.7 of its current value. So Groundwater use $\mathrm{t}_{+1}=$ Groundwater use $\mathrm{t}+^{+}$ (Groundwater use $\mathrm{t} * 0.7$ ).

2.5) At the end of each action list, if agent has no other actions left, it has to sell off its farms to large-farmers and leave if it belongs to small or medium holders. Large farmers have to relocate their farms to out of the region at the end of their action list. 


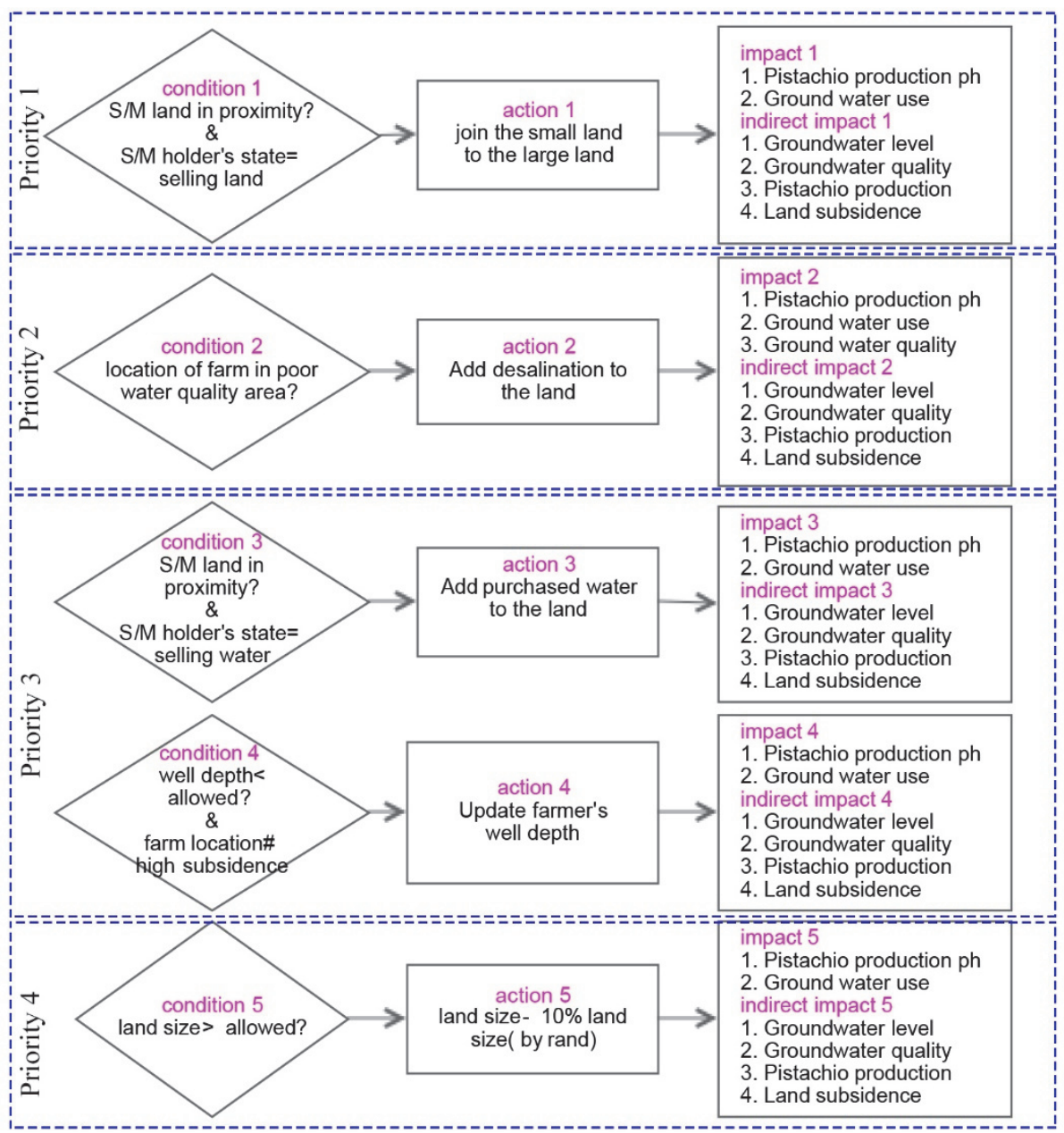

Figure 5. Conditions, actions and impacts for large-holders' set of actions 


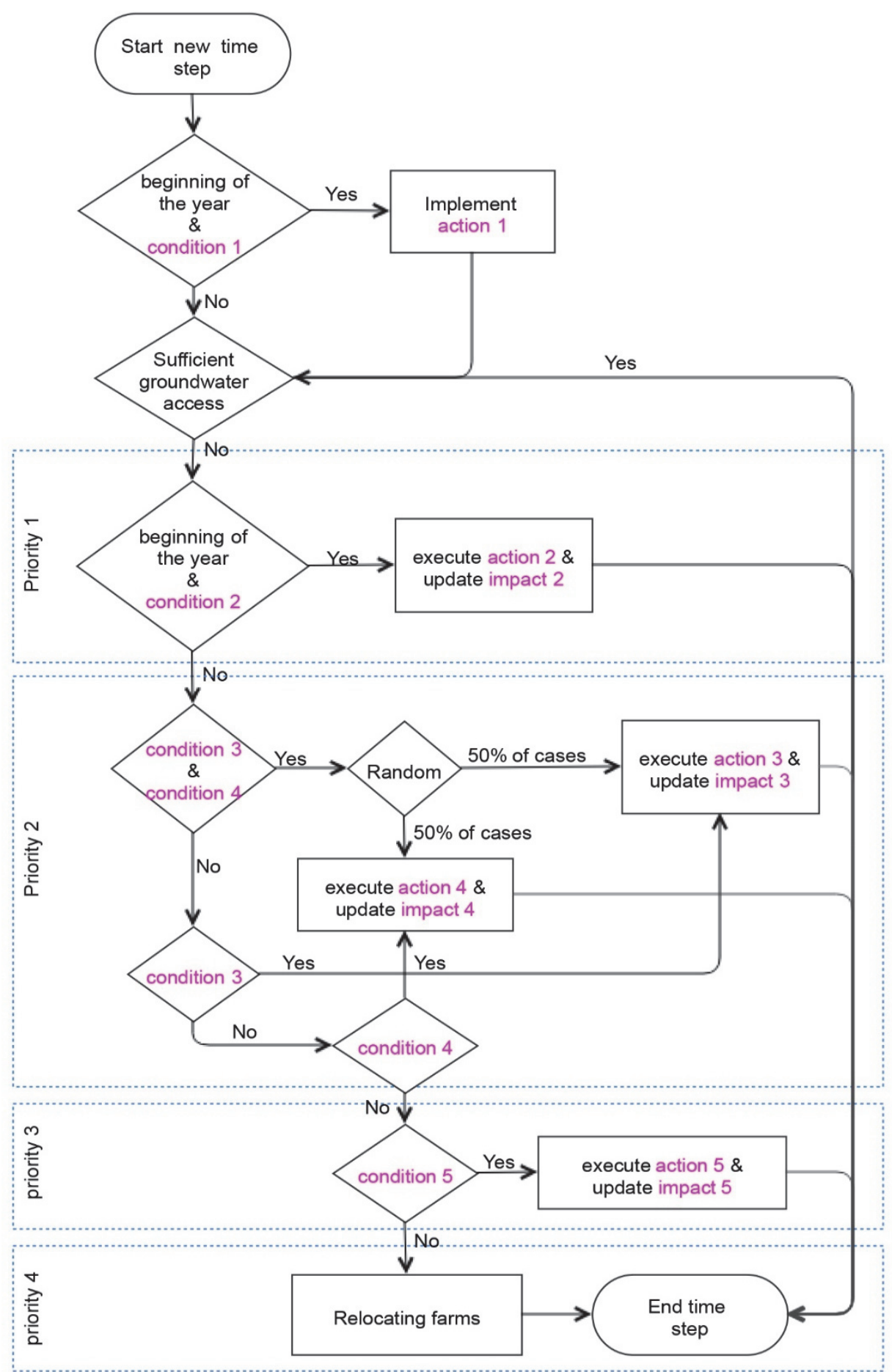

Figure 6. UML activity diagram of large farmers' behavioral rules in each time step 
Table 2. Conditions, actions and impacts for large-farmers' set of actions

\begin{tabular}{|c|c|c|c|}
\hline Action name & Conditions & Execution & impacts \\
\hline $\begin{array}{l}\text { 1.Buying } \\
\text { small/medium } \\
\text { farms }\end{array}$ & $\begin{array}{l}\text { Small land in } \\
\text { neighborhood } \\
\& \\
\text { Small-farmer is } \\
\text { willing to sell-off } \\
\text { land } \\
\end{array}$ & $\begin{array}{l}\text { Change owner } \\
\text { of small farm } \\
\text { from small- } \\
\text { farmer to the } \\
\text { large-farmer in } \\
\text { neighbor }\end{array}$ & $\begin{array}{l}\text { On small-farm patches } \\
\text { 1. GW-use }=\mathrm{GW} \text {-use } * \\
0.1 \\
\text { 2. Productivity } \mathrm{t}+1= \\
\text { Productivity } \mathrm{t}+ \\
\text { Productivity }(\mathrm{t})^{*} 0.9\end{array}$ \\
\hline $\begin{array}{l}\text { 2. Desalination } \\
\text { (one-time } \\
\text { action) }\end{array}$ & $\begin{array}{c}\text { Action } 2 \text { is } \\
\text { available (has not } \\
\text { been executed } \\
\text { before) } \\
\& \\
\text { Location of farm in } \\
\text { poor GW quality } \\
\text { area }\end{array}$ & $\begin{array}{l}\text { Set action } 2 \text { not } \\
\text { available for } \\
\text { next steps }\end{array}$ & $\begin{array}{l}\text { 1. GW-use } t+1=G W- \\
\text { use }_{t}+\mathrm{GW}_{\text {-use }}(\mathrm{t}) \\
0.7 \\
\text { 2. Productivity }{ }_{\mathrm{t}+1}= \\
\text { Productivity }_{\mathrm{t}}+ \\
\text { Productivity }(\mathrm{t})^{*} * 0.9\end{array}$ \\
\hline $\begin{array}{l}\text { 3. Water } \\
\text { purchase }\end{array}$ & $\begin{array}{c}\text { Small land in } \\
\text { neighborhood? } \\
\& \\
\text { Small-farmer is } \\
\text { willing to sell-off } \\
\text { water }\end{array}$ & $\begin{array}{l}\text { Add purchased } \\
\text { water to the } \\
\text { properties of } \\
\text { farm-cluster }\end{array}$ & 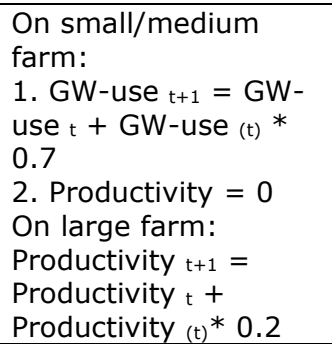 \\
\hline $\begin{array}{l}\text { 4. Deepening } \\
\text { wells }\end{array}$ & $\begin{array}{c}\text { Well depth < } \\
\text { allowed well depth } \\
\& \\
\text { Farm location is } \\
\text { not in high } \\
\text { subsidence areas }\end{array}$ & $\begin{array}{l}\text { Update the } \\
\text { depth of the well }\end{array}$ & $\begin{array}{l}\text { 1. GW-use } \mathrm{t}_{\mathrm{1}}=\mathrm{GW}- \\
\text { use } \mathrm{t}+\mathrm{GW} \text {-use }(\mathrm{t}) * \\
0.5 \\
\text { 2. Productivity }_{\mathrm{t}+1}= \\
\text { Productivity }_{\mathrm{t}}+ \\
\text { Productivity }(\mathrm{t})^{*} 0.7\end{array}$ \\
\hline $\begin{array}{l}\text { 5. Irrigation } \\
\text { area reduction }\end{array}$ & $\begin{array}{l}\text { Land size }>=50 \% \\
\text { initial land size } \\
\text { (large farmers } \\
\text { accept to shrink up } \\
\text { to } 50 \% \text { of their } \\
\text { lands) }\end{array}$ & $\begin{array}{l}\text { Change } 10 \% \text { of } \\
\text { farm patches to } \\
\text { no-farm }\end{array}$ & $\begin{array}{l}\text { On dried patches: } \\
\text { 1.GW-use }=0 \\
\text { 2. Productivity }=0 \\
\text { On farm patches: } \\
\text { 1. GW-use } t+1=G W- \\
\text { use } t+G W \text {-use }(\mathrm{t}) *(- \\
0.1) \\
\text { 2. Productivity }{ }_{\mathrm{t}+1}= \\
\text { Productivity }_{\mathrm{t}}+ \\
\text { Productivity }(\mathrm{t})^{+} * 0.5\end{array}$ \\
\hline $\begin{array}{l}\text { 6. Relocating } \\
\text { farms }\end{array}$ & $\begin{array}{l}\text { No other available } \\
\text { actions }\end{array}$ & $\begin{array}{l}\text { Change all farm- } \\
\text { patches to no- } \\
\text { farm }\end{array}$ & $\begin{array}{l}\text { 1.GW-use }=0 \\
\text { 2. Productivity }=0\end{array}$ \\
\hline
\end{tabular}


Table 3. Conditions, actions and impacts for medium-farmers' set of actions

\begin{tabular}{|c|c|c|c|}
\hline Action name & Conditions & Execution & Impacts \\
\hline $\begin{array}{l}\text { 1.Deepening } \\
\text { wells }\end{array}$ & $\begin{array}{c}\text { Well depth < } \\
\text { allowed well depth } \\
\& \\
\text { Farm location is } \\
\text { not in high } \\
\text { subsidence areas }\end{array}$ & $\begin{array}{l}\text { Update the depth } \\
\text { of the well }\end{array}$ & $\begin{array}{l}\text { 1. GW-use } \mathrm{t}+1=\mathrm{GW}- \\
\text { use }_{\mathrm{t}}+\mathrm{GW} \text { - } \mathrm{use}_{(\mathrm{t})} * 0.5 \\
\text { 2. Productivity }{ }_{\mathrm{t}+1}= \\
\text { Productivity }^{\mathrm{n}}+ \\
\text { Productivity }(\mathrm{t})^{\mathrm{n}}{ }^{0.9}\end{array}$ \\
\hline $\begin{array}{l}\text { 2.Integrating } \\
\text { farming } \\
\text { (By rand: } \\
\text { change } \\
\text { farmer's state } \\
=\text { integrating } \\
\text { farm) }\end{array}$ & $\begin{array}{l}\text { Medium land in } \\
\text { neighbor? } \\
\& \\
\text { Neighbor medium- } \\
\text { farmer is willing to } \\
\text { land integration }\end{array}$ & & 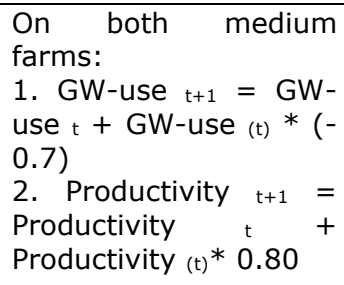 \\
\hline $\begin{array}{l}\text { 3. Irrigation } \\
\text { system } \\
\text { modification }\end{array}$ & $\begin{array}{c}\text { Act } 3=1 \\
\text { (available) } \\
\& \\
\text { Farmer's land doc } \\
=1 \text { (available) }\end{array}$ & $\begin{array}{l}\text { Add irrigation- } \\
\text { modification to } \\
\text { the properties of } \\
\text { farm-cluster } \\
\text { Set act } 3=0\end{array}$ & $\begin{array}{l}\text { 1. GW-use }{ }_{t+1}=G W- \\
\text { use } t+G W \text {-use }(t) *(- \\
0.6) \\
\text { 2. Productivity }{ }^{t+1}= \\
\text { Productivity }{ }^{t}+ \\
\text { Productivity }(\mathrm{t}) * 0.75\end{array}$ \\
\hline $\begin{array}{l}\text { 4. Irrigation } \\
\text { area reduction }\end{array}$ & $\begin{array}{l}\text { Land size }>=60 \% \\
\text { initial land size }\end{array}$ & $\begin{array}{l}\text { Change } 10 \% \text { of } \\
\text { farm clusters to } \\
\text { no-farm }\end{array}$ & $\begin{array}{l}\text { On dried patches: } \\
\text { 1.GW-use }=0 \\
\text { 2. Productivity }=0 \\
\text { On farm patches: } \\
\text { 1. GW-use } \mathrm{t+1}=\mathrm{GW}- \\
\text { use } \mathrm{t}+\mathrm{GW} \text {-use }{ }_{(\mathrm{t})} *(- \\
0.15) \\
\begin{array}{l}\text { 2. Productivity }{ }_{\mathrm{t}+1}= \\
\text { Productivity }{ }^{\mathrm{t}}+ \\
\text { Productivity }(\mathrm{t})\end{array} 0^{\mathrm{H}}+\end{array}$ \\
\hline $\begin{array}{l}5 . \text { Sell land } \\
\text { and relocation }\end{array}$ & $\begin{array}{l}\text { Farmer's } \\
\text { vulnerability } \\
\text { very high }\end{array}$ & $\begin{array}{l}\text { Change all farm- } \\
\text { cluster to no- } \\
\text { farm }\end{array}$ & $\begin{array}{l}\text { 1.GW-use }=0 \\
\text { 2. Productivity }=0\end{array}$ \\
\hline
\end{tabular}


Table 4. Conditions, actions and impacts for small-holders' set of actions

\begin{tabular}{|c|c|c|c|}
\hline Action name & Conditions & Execution & impacts \\
\hline $\begin{array}{l}\text { 1.Irrigation } \\
\text { system } \\
\text { modification }\end{array}$ & $\begin{array}{c}\text { Act } 3=1 \\
\text { (available) } \\
\& \\
\text { Farmer's land doc } \\
=1 \text { (available) } \\
\& \\
\text { Random }>33 \%\end{array}$ & $\begin{array}{l}\text { Add irrigation- } \\
\text { modification to } \\
\text { the properties of } \\
\text { farm-cluster } \\
\text { Set act } 3=0\end{array}$ & $\begin{array}{l}\text { 1. GW-use } \mathrm{t}+1=\mathrm{GW}- \\
\text { use }_{\mathrm{t}}+\mathrm{GW} \text {-use }(\mathrm{t}) *(- \\
0.7)^{-} \\
\text {2. Productivity } \mathrm{t+1}= \\
\text { Productivity }_{\mathrm{t}}+ \\
\text { Productivity }_{(\mathrm{t})} * 0.7\end{array}$ \\
\hline $\begin{array}{l}\text { 2.well's turn } \\
\text { off }\end{array}$ & & & 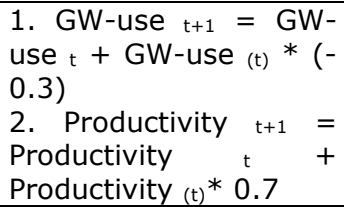 \\
\hline $\begin{array}{l}\text { 4. Irrigation } \\
\text { area } \\
\text { reduction }\end{array}$ & $\begin{array}{l}\text { Land size }>=70 \% \\
\text { initial land size }\end{array}$ & $\begin{array}{l}\text { Change } 10 \% \text { of } \\
\text { farm clusters to } \\
\text { no-farm }\end{array}$ & 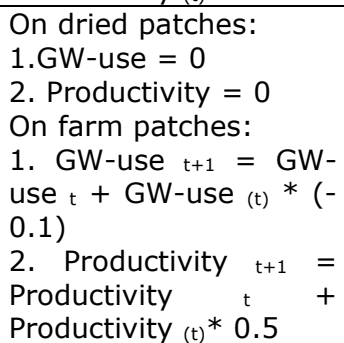 \\
\hline $\begin{array}{l}\text { 5. Sell land } \\
\text { and relocation }\end{array}$ & $\begin{array}{l}\text { Farmer's } \\
\text { vulnerability = very } \\
\text { high }\end{array}$ & $\begin{array}{l}\text { Change all farm- } \\
\text { cluster to no-farm }\end{array}$ & $\begin{array}{l}\text { 1.GW-use }=0 \\
\text { 2. Productivity }=0\end{array}$ \\
\hline
\end{tabular}

Example: Large farmers have six possible adaptive action.

Action 1) is "buying small/medium farms" for which there are two conditions: there should be at least one small/medium land in the neighborhood of that agent's patch and that small farmer's state of "selling land" should be "on" that shows small farmer is willing to sell his/her land. When both of these conditions are confirmed, the small patch will be added to the large patch. By executing of this action 1. Ground water use of the small farms decreases by $90 \%$, and 2. Pistachio production of small land increases by $90 \%$.

Action 2) Desalination, for which there are two conditions: 1. this action should have not been implemented before, so, "desalination" property of farm should be equal to 0 , and 2 . the farm should be located in poor quality area. When both of these conditions are confirmed, the desalination is added to the land (desalination $=1$ ), meaning not available for next time steps. By executing of this action 1. Pistachio production increases by $90 \%$, and 2 . Ground water use increases by $70 \%$.

Action 3) Purchasing water, for which there are two conditions: there should be at least one small land in the neighborhood and that small farmer's state of "selling water" should be "on" that shows small-farmer is willing to sell his/her water. This property is related to the small farmer's level of vulnerability. When 
both of these conditions are confirmed, the "purchased water" gets added to the property of land. By executing of this action 1 . Small/medium farm get no pistachio production, 2. Ground water use of small farms increases by $70 \%$, and 3. Pistachio production of large farmers increases by $20 \%$.

Action 4) deepening wells, for which there are two conditions: wells depth should not be equal or lower than the permitted depth and farm's location should not be in very high land subsidence areas. When both of these conditions are confirmed the wells depth gets update. By execution of this action 1) Ground water use increases by $50 \%$, and 2) pistachio production increases by $70 \%$.

Action 5: for which there is only one condition. As the last action before selling or relocating the lands, farmers start to shrink their farming area to increase efficiency of their production per hectare. However the land area reduction keeps happening till farmers still have some benefit of their lands. Otherwise they prefer to sell off land/water or relocate their land which can be more beneficial than shrinking and farming in the smaller lands. The threshold of shrinking lands is approximately $30 \%$ for small-holders, $40 \%$ for mediumholders and $50 \%$ for large-holders from FCM models. Therefore, if the agent's patches is bigger than minimum possible land ( $\left.p>70 \% / 60 \% / 50 \% * p_{1}\right)$ then the agent's patches reduces by $10 \%\left(p_{n+1}=90 \% * p_{1}\right)$. By execution of this action 1) groundwater use and pistachio production of dried patches get equal to zero, 2) groundwater use of not dried patches decreases by $10 \%$, and pistachio production of not dried patches increases by $50 \%$. 


\section{Appendix 9. Sensitivity Analysis}

\begin{tabular}{|c|c|c|c|}
\hline Parameter & Description & $\begin{array}{l}\text { Source of } \\
\text { data }\end{array}$ & $\begin{array}{c}\text { Nominal } \\
\text { value } \\
(\mathrm{L} / \mathrm{M} / \mathrm{S})\end{array}$ \\
\hline Actions $\rightarrow$ GW use & \multirow{9}{*}{$\begin{array}{l}\text { Impact of } \\
\text { different actions } \\
\text { on groundwater } \\
\text { use for three } \\
\text { groups of large, } \\
\text { medium and small } \\
\text { farmers }\end{array}$} & \multirow{9}{*}{ FCM } & \\
\hline 1. Buying land $\rightarrow$ GW use (L) & & & -0.9 \\
\hline 2. Desalination $\rightarrow \mathrm{GW}$ use $(\mathrm{L})$ & & & 0.7 \\
\hline 3. Water purchasing $\rightarrow \mathrm{GW}$ use (L) & & & 0.7 \\
\hline 4. Deepening wells $\rightarrow \mathrm{GW}$ use $(\mathrm{L}, \mathrm{M})$ & & & $0.5 / 0.4$ \\
\hline $\begin{array}{l}\text { 5. Shrinking lands } \rightarrow \mathrm{GW} \text { use } \\
\qquad(L, M, S)\end{array}$ & & & $\begin{array}{c}-0.1 /- \\
0.15 /- \\
0.1\end{array}$ \\
\hline $\begin{array}{l}\text { 6. Irrigation change } \rightarrow \mathrm{GW} \text { use } \\
(\mathrm{M}, \mathrm{S})\end{array}$ & & & $\begin{array}{l}-0.6 /- \\
0.7\end{array}$ \\
\hline 7. Farm integration $\rightarrow \mathrm{GW}$ use (M) & & & -0.7 \\
\hline 8. Well's turn off $\rightarrow \mathrm{GW}$ use $(\mathrm{M}, \mathrm{S})$ & & & $\begin{array}{l}-0.3 /- \\
0.1\end{array}$ \\
\hline 9. $\mathrm{GW}$ access $\rightarrow \mathrm{GW}$ use $(L, M, S)$ & $\begin{array}{c}\text { Impact of } \\
\text { changing } \\
\text { groundwater } \\
\text { access limitation } \\
\text { (GW level - Well's } \\
\text { depth) on } \\
\text { groundwater use }\end{array}$ & $\begin{array}{l}\text { Stakeholders } \\
\text { ' Estimation }\end{array}$ & \\
\hline
\end{tabular}

Buyinglands-GWU-R4

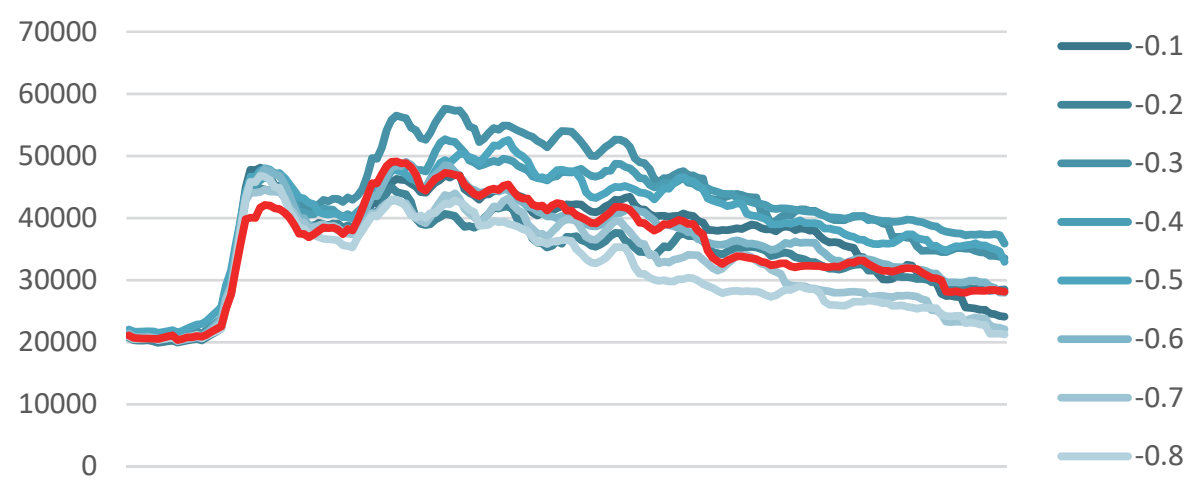

Figure 1: Changing impact of buying lands on groundwater use - Region 4 


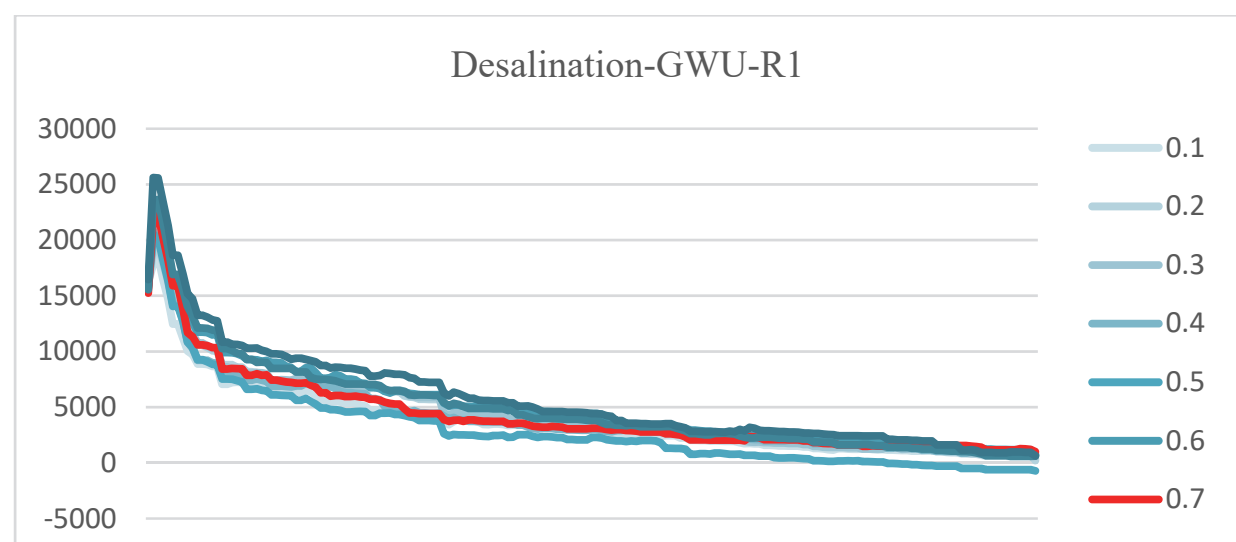

Figure 2: Changing impact of desalination on groundwater use - Region 1

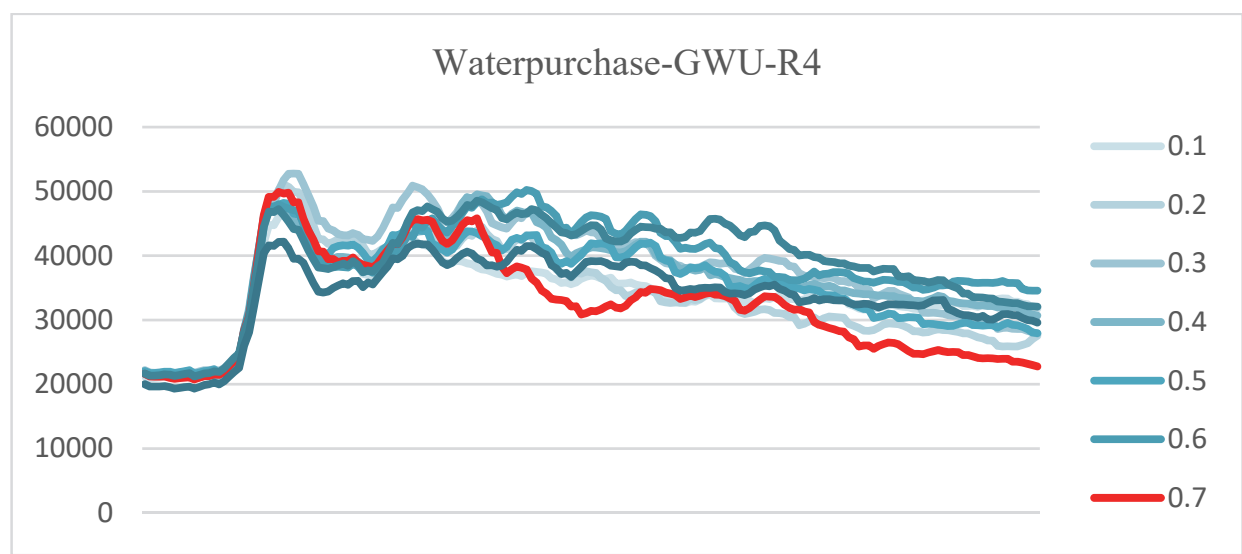

Figure 3: Changing impact of water purchase on groundwater use - Region 4

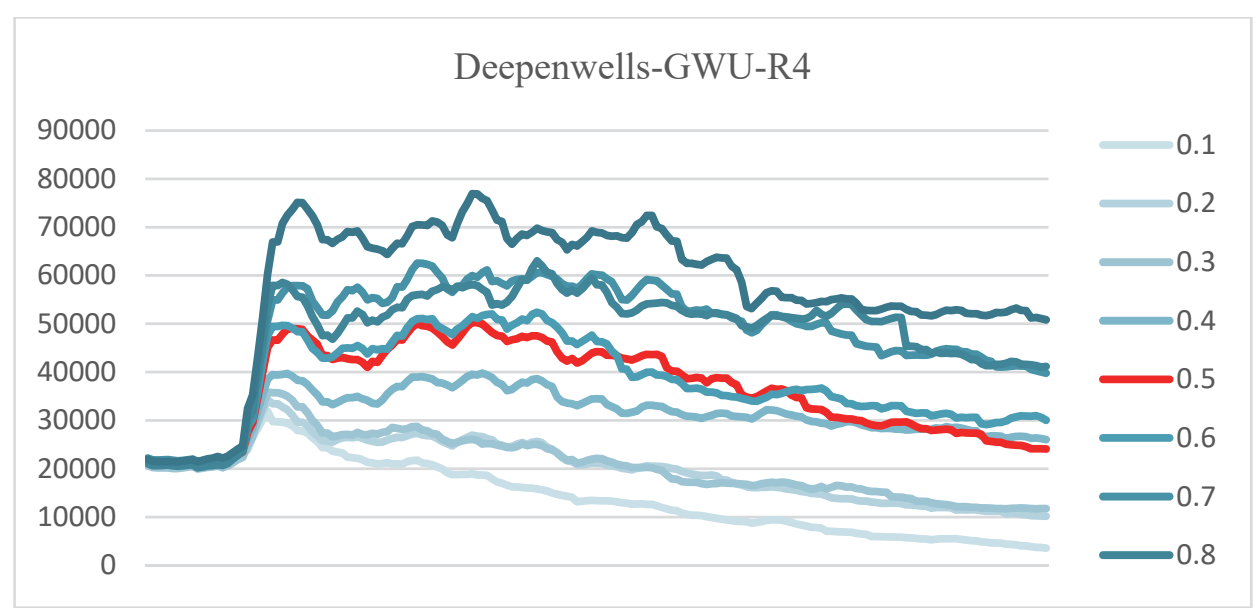

Figure 4: Changing impact of deepening wells on groundwater use - Region 4 


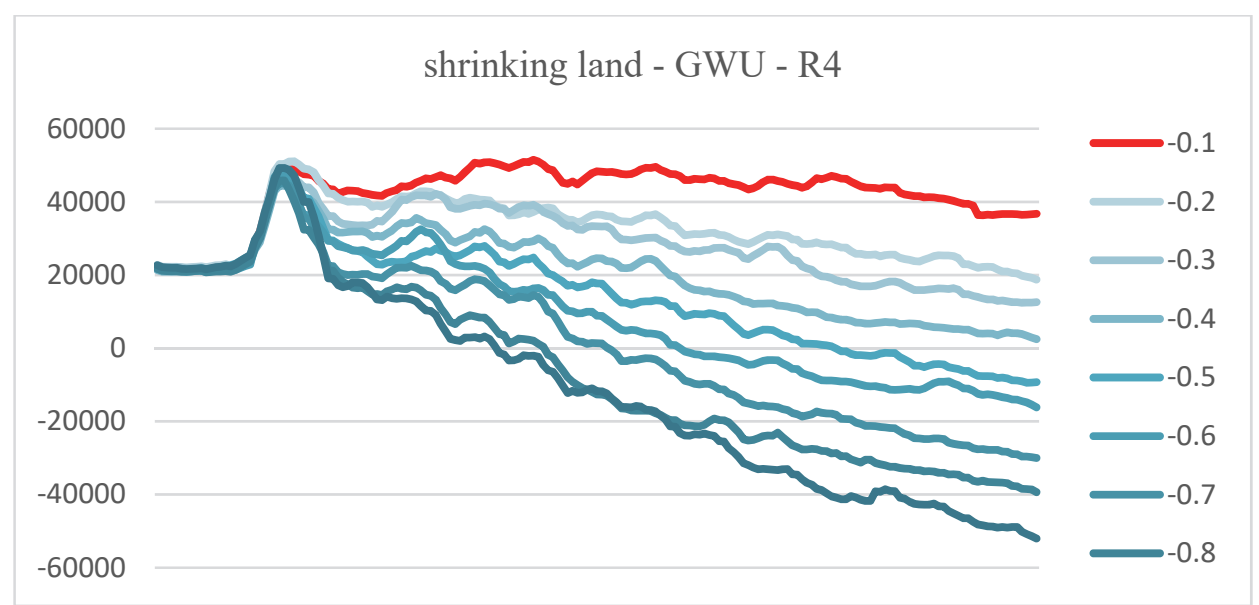

Figure 5: Changing impact of shrinking lands on groundwater use - Region 4

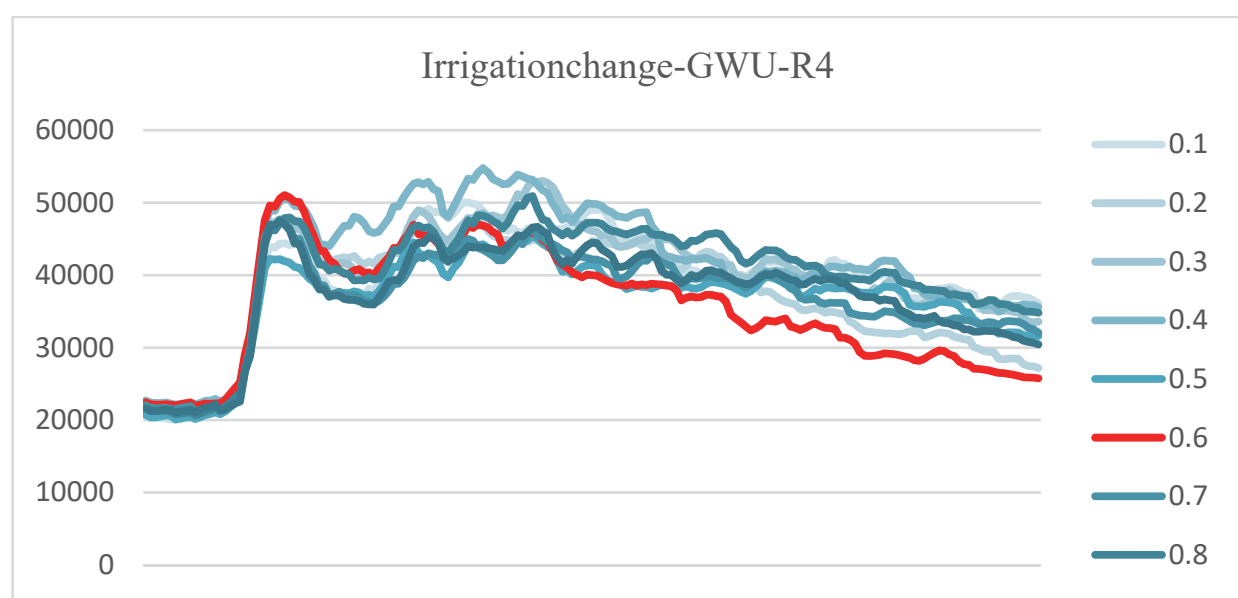

Figure 6: Changing impact of irrigation change on groundwater use - Region 4

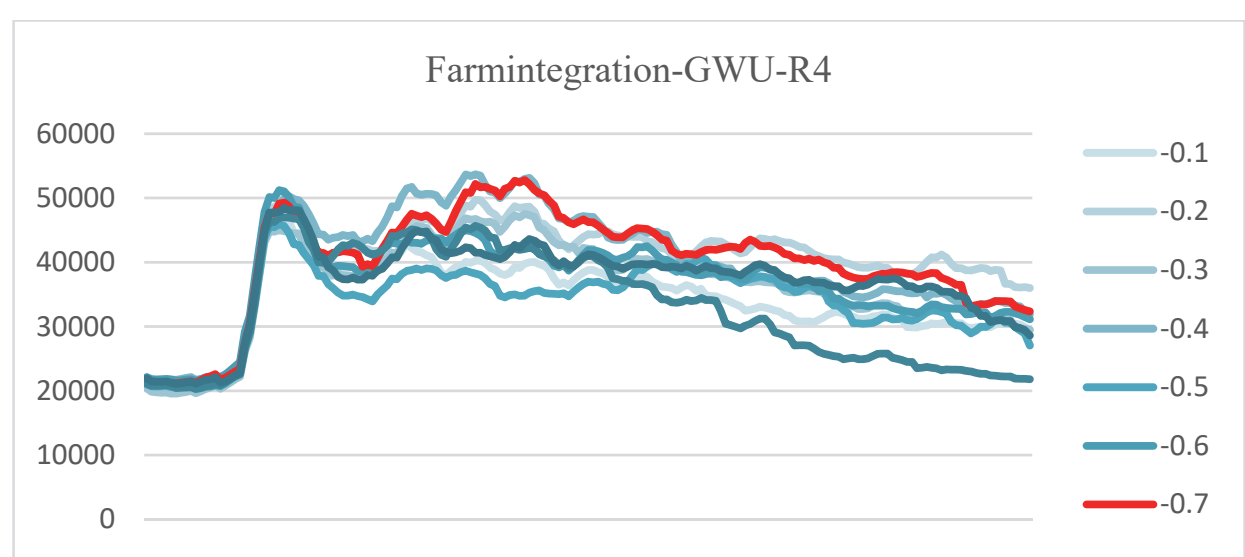

Figure 7: Changing impact of farm integration on groundwater use - Region 4 


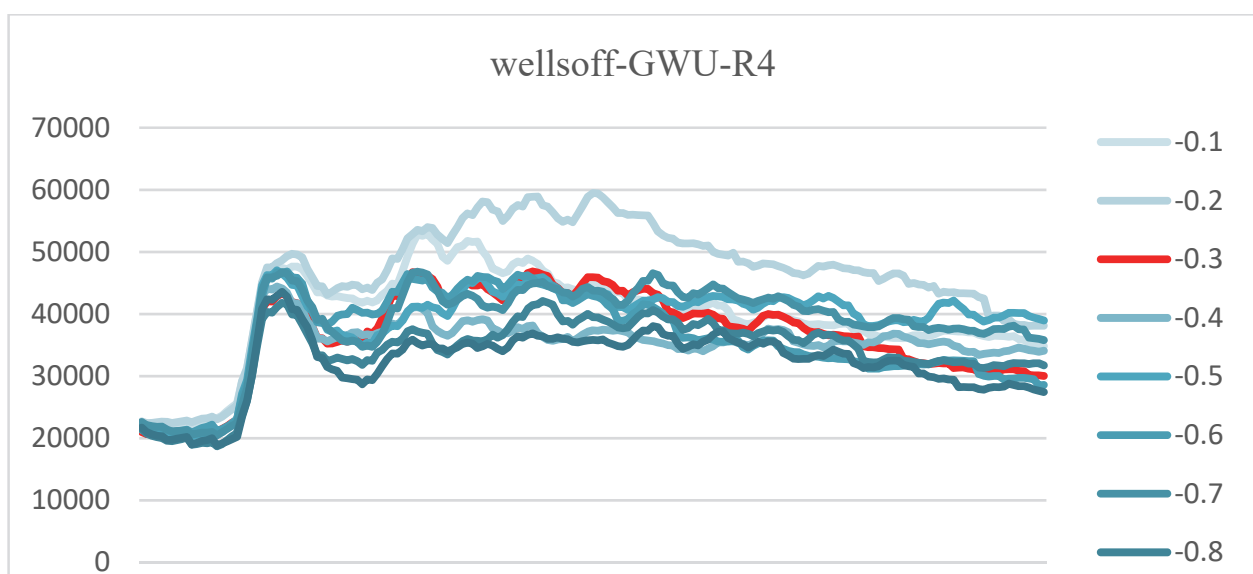

Figure 8: Changing impact of wells-off on groundwater use - Region 4

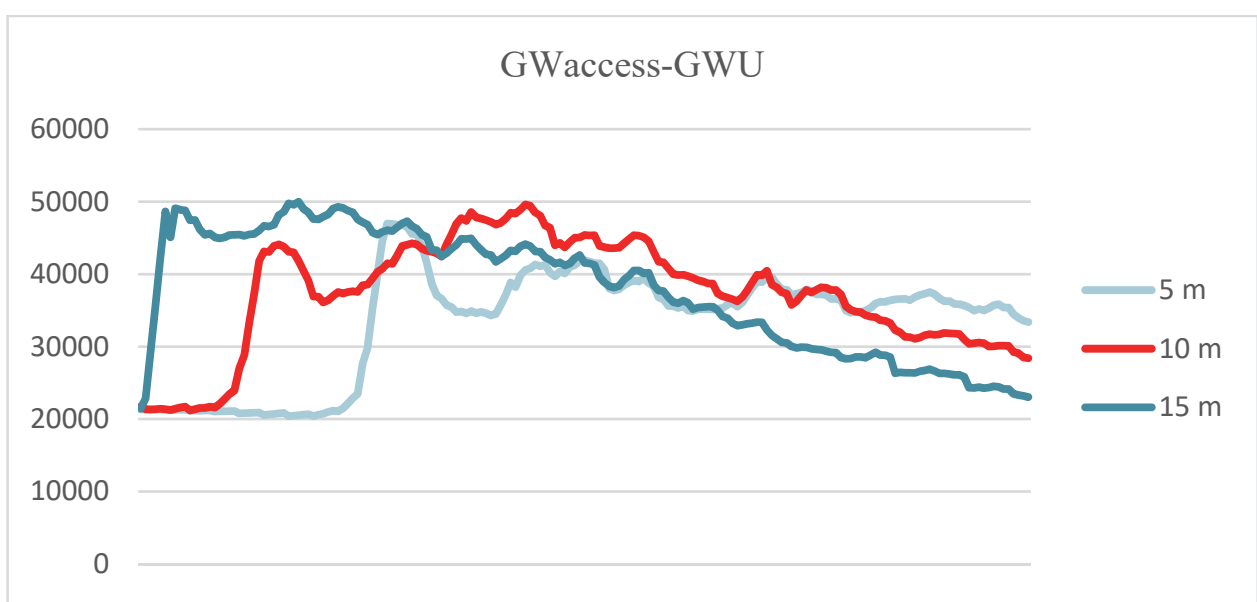

Figure 9: Changing groundwater access limitation - Region 4

\section{Appendix 10. Data-driven variables and their correlated connections}

Table 1: Connections with data-driven weights and their values

Connections

Climate change $\rightarrow$ Pistachio production

Measured data correlation

Groundwater exploitation $\rightarrow$ Groundwater level

$\mathbf{- 0 . 4}$

Groundwater level $\rightarrow$ Groundwater quality

$-0.8$

Groundwater level $\rightarrow$ Land subsidence

0.3

Groundwater level $\rightarrow$ Land subsidence

$-0.9$

Groundwater level $\rightarrow$ Pistachio production

0.6

Groundwater quality $\rightarrow$ Pistachio production

0.1 
Table 2. Description of variables with available time-series data

\begin{tabular}{|c|c|c|c|}
\hline $\begin{array}{l}\text { Variable } \\
\text { name }\end{array}$ & Description & Years of data & Source \\
\hline $\begin{array}{l}\text { Climate } \\
\text { change } \\
\text { (precipitatio } \\
\mathrm{n} \text { and heat) }\end{array}$ & $\begin{array}{l}\text { Annual precipitation } \\
\text { (mm) and average } \\
\text { monthly maximum } \\
\text { temperature }\end{array}$ & $\begin{array}{l}1982 \text { to } 2016 \\
\text { annually }\end{array}$ & $\begin{array}{l}\text { Iran Meteorological } \\
\text { Organization (IRIMO) }\end{array}$ \\
\hline $\begin{array}{l}\text { Groundwater } \\
\text { exploitation }\end{array}$ & $\begin{array}{l}\text { Sum of the annual } \\
\text { groundwater } \\
\text { exploitation by total } \\
\text { wells (mm3) }\end{array}$ & $\begin{array}{l}\text { 1971- 1973- 1974- } \\
\text { 1975- 1976- 1981- } \\
\text { 1983- 1986- 1989- } \\
1993-1997-2005- \\
2006\end{array}$ & $\begin{array}{l}\text { Official reports, Water } \\
\text { research institute, } \\
\text { Ministry of Energy }\end{array}$ \\
\hline $\begin{array}{l}\text { Groundwater } \\
\text { level }\end{array}$ & $\begin{array}{l}\text { Average of the } \\
\text { annual groundwater } \\
\text { level of the whole } \\
\text { Rafsanjan }\end{array}$ & $\begin{array}{l}1983 \text { to } 2013 \\
\text { annually }\end{array}$ & $\begin{array}{l}\text { Official reports, Iran } \\
\text { water resources } \\
\text { Management Company } \\
\text { http://wrbs.wrm.ir/ }\end{array}$ \\
\hline $\begin{array}{l}\text { Groundwater } \\
\text { quality }\end{array}$ & $\begin{array}{l}\text { Average of the } \\
\text { annual Electrical } \\
\text { Conductivity (EC) of } \\
\text { groundwater in } \\
\text { Rafsanjan }\end{array}$ & $\begin{array}{l}1998 \text { to } 2015 \\
\text { annually }\end{array}$ & $\begin{array}{l}\text { Official reports, Iran } \\
\text { water resources } \\
\text { Management Company } \\
\text { http://wrbs.wrm.ir/ }\end{array}$ \\
\hline $\begin{array}{l}\text { Pistachio } \\
\text { production }\end{array}$ & $\begin{array}{l}\text { Sum of production } \\
\text { in the whole } \\
\text { Rafsanjan }\end{array}$ & $\begin{array}{l}1982 \text { to } 2015 \\
\text { annually }\end{array}$ & $\begin{array}{l}\text { Iran Pistachio } \\
\text { Association } \\
\text { iranpistachio.org/fa/sa } \\
\text { mple/before }\end{array}$ \\
\hline $\begin{array}{l}\text { Land } \\
\text { subsidence }\end{array}$ & & $\begin{array}{l}2004 \text { to } 2016 \\
\text { annually }\end{array}$ & $\begin{array}{l}\text { Scientific studies } \\
\text { (Motagh et al., 2017) }\end{array}$ \\
\hline
\end{tabular}

\section{Appendix 10: Oral Consent Script}

At the beginning of each interview, the interviewees were informed about the purposes of the study, confidentiality of their information, and outcome of the interview. Their oral informed consents were obtained and recorded using a digital recorder (alongside the whole interview). Below is the (translated) oral consent script of our interviews and the main questions for discussions.

\section{Oral Consent Script}

This interview is designed to collect data for the PhD project of me, [name of researcher] in [name of university and country] about the problem of water scarcity in Rafsanjan. The outputs of this interview will be exclusively used for academic purposes. In any report on the results of this research, your identity will remain anonymous and details of your interview will be aggregated with others for presentation in the reports.

During the interview, you will be asked questions and based on your answers I draw a mind map like this one (an FCM irrelevant to the topic of research is shown to the interviewee) on the paper, which you can view and comment on. I would like to record this interview if you do not mind. I may also 
contact you for follow-up questions or clarification. No-one other than me (the first author) will have access to raw data i.e. your mind maps and detail information.

You don't have to agree to take part; you can ask me any questions you want before or throughout; you can also withdraw at any stage without giving a reason.

Do you give your permission for me to interview and audio record you, and re-contact you to clarify information?

\section{Guiding Questions}

Name, age, gender:

Location and size of the farm:

Other source of income:

$\mathrm{Nr}$ of wells and depth of each well:

1. What have been the main causes of water scarcity in your region/farm?

2. What have been the main impacts of water scarcity in your region/farm?

3. How much has each of these variables caused an increase or decrease in other variables?

4. What have been your adaptive actions to combat water scarcity in your farm

5. What have been the conditions to implement each action?

6. How much has each action impacted other variables mentioned earlier?

\section{Ending Questions}

Would you please provide me with your phone number for follow-up questions?

If you like to see the aggregated mind-map of all farmers please give me your email address (or send me the email address of a person you know). 


\section{Appendix 11: Demographic characteristics of interviewees}

\begin{tabular}{|c|c|c|c|}
\hline $\begin{array}{l}\text { Demographic } \\
\text { Variable }\end{array}$ & Large Farmers & Medium Farmers & Small Farmers \\
\hline Number & 20 & 20 & 20 \\
\hline Age & \multicolumn{3}{|c|}{$M=65.7, S D=10.6$, Range $=32-93$} \\
\hline Gender & $M=18, F=2$ & $M=20, F=0$ & $M=20, F=0$ \\
\hline Location of farms & $\begin{array}{l}\text { Sub-region } 1=3 \\
\text { Sub-region } 2=3 \\
\text { Sub-region } 3=2 \\
\text { Sub-region } 4=3 \\
\text { Sub-region } 5=3 \\
\text { Sub-region } 6=3 \\
\text { Sub-region } 7=3\end{array}$ & $\begin{array}{l}\text { Sub-region } 1=3 \\
\text { Sub-region } 2=3 \\
\text { Sub-region } 3=2 \\
\text { Sub-region } 4=3 \\
\text { Sub-region } 5=3 \\
\text { Sub-region } 6=3 \\
\text { Sub-region } 7=3\end{array}$ & $\begin{array}{l}\text { Sub-region } 1=3 \\
\text { Sub-region } 2=3 \\
\text { Sub-region } 3=2 \\
\text { Sub-region } 4=3 \\
\text { Sub-region } 5=3 \\
\text { Sub-region } 6=3 \\
\text { Sub-region } 7=3\end{array}$ \\
\hline Farm Size & $\begin{array}{l}\text { Range }=80-250 \\
\text { ha } \\
M=112\end{array}$ & $\begin{array}{l}\text { Range }=15-80 \text { ha } \\
M=47\end{array}$ & $\begin{array}{l}\text { Range }=0.5-15 \text { ha } \\
M=5\end{array}$ \\
\hline $\begin{array}{l}\text { Other source of } \\
\text { income }\end{array}$ & Yes $=18, \mathrm{No}=2$ & Yes $=11, \mathrm{No}=9$ & $\mathrm{Yes}=7, \mathrm{No}=13$ \\
\hline
\end{tabular}

\section{Appendix 12: an example of FCM drawn by one of stakeholders}

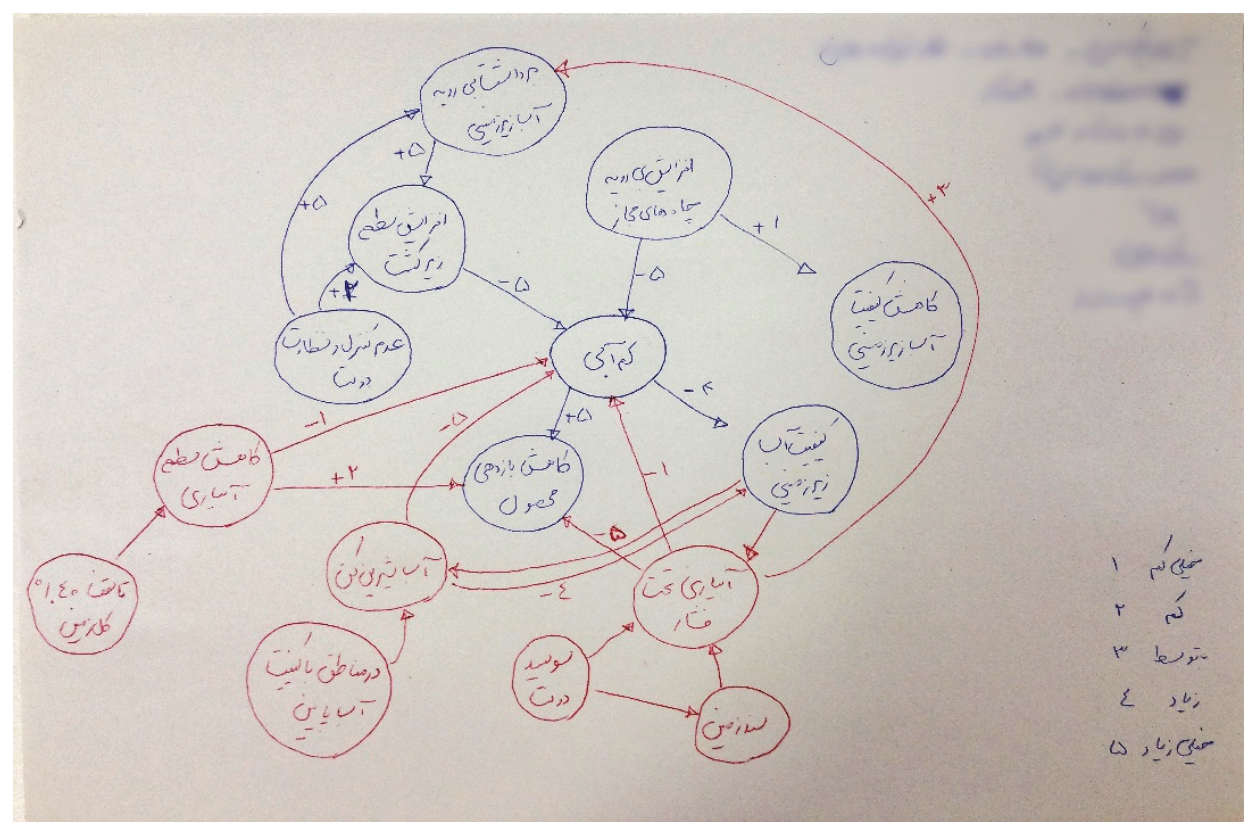




\section{Summary}

Climate change and global warming are noticeably increasing the losses and damages of natural resources, including fish stocks, lakes, water resources, forests, and farms. Climate change adaptation is a response to such environmental changes by attempting to reduce the vulnerability of social and ecological sub-systems to changes of temperature, rainfall, sea level, etc. Policies designed for climate change adaptation should consider both social and ecological systems impacted by climate change as well as the interaction between such systems, which calls for a Social-Ecological System (SES) perspective.

In this thesis, we argue that participatory policy analysis methods are crucial for decision-making related to climate change adaptation in SESs. Such methods involve a diversity of knowledge, perceptions, preferences, and decision-making of people managing or depending on the natural resources in the process of policy preparation. The participatory policy analysis approach has evolved in response to the failures of traditional policy analysis approaches that place emphasis on formal (quantitative) modelling, rational planning and cost-benefit analysis to find solutions for all sorts of complex problems. We argue that the participatory approach helps policy makers to address the most important features of an SES, i.e., complexity, dynamics and uncertainty, in their policy options analysis.

The main objective of this research is to develop and demonstrate participatory policy analysis methods to support policy-making in SESs' environmental challenges. The research is built upon a case study of a farming community facing water scarcity in Rafsanjan, Iran. To achieve the objective, a combination of participatory methods in a specific sequence is designed to integrate farmers' and policy makers' knowledge, perception, preferences and decision-making in the process of policy making for water scarcity in Rafsanjan. The Driver-Pressure-State-Impact-Response (DPSIR) framework is used in step 1 to categorize and structure the complex SES problem of water scarcity in the case study. In step 2, the DPSIR framework is combined with a participatory Fuzzy Cognitive Mapping method (FCM, i.e. a knowledge coproduction method) to systematically collect the knowledge and perceptions of farmers and policy makers on the data-scarce part of the system and to represent these in a semi-quantitative model. In step 3, the qualitative knowledge produced by participatory FCM is combined with quantitative data to develop a mixed-FCM representing complex SES related to water scarcity. In step 4, the outcome of the mixed FCM is used as an input to develop an Agent-Based Model (ABM) to model the decisions and actions of farmers and simulate the macro-level patterns of the system that emerges from individual behaviour. Finally, the impact of government policy options are simulated by 
integrating knowledge, perceptions and preferences of stakeholders represented in FCM models, and their decisions and actions represented by ABM.

The outcome of this research is a policy support toolbox that provides four different methods, each one addresses different problem contexts or policy objectives related to climate change adaptation for SESs. DPSIR is a problem structuring framework that helps to categorize and understand different aspects of the problem before policy making. The Perceived-FCM is valuable in predicting stakeholders' responses to new policies. The Mixed-FCM is useful in simulating and analysing impacts of policy options in the multi-factorial SESs, for which both subjective and objective knowledge is relevant for a better understanding of system's behaviour. Finally, the FCM-based ABM is useful in simulating impacts of policy options by considering actual human decisions and actions as well as the multifactor behaviour in the SESs.

Moreover, the outcome of this study contributes in bridging gaps in 1) integrated use of qualitative and quantitative evidence in SES modelling, 2) modelling the micro-level and macro-level behaviour of the SESs, and 3) the combination of actor-based and factor-based approaches in SES modelling. Furthermore, this study introduces policy support methods that cover the main features of a complex SES i.e. causality, feedback loops, social-spatial heterogeneity, and temporal dynamics.

Finally, the outcomes of this research might be useful for three groups of people: 1 . researchers who have an interest in novel methods to model SESs, 2. practitioners who can use the methods for participatory policy option analysis, and 3. students who can learn from the step-wise approach developed in this research for their own future studies. 


\section{Samenvatting}

Klimaatverandering en de opwarming van de aarde leiden tot een aantoonbare toename van verliezen van en schaden aan natuurlijke hulpbronnen waaronder de visstand, meren, water bronnen en voorraden, bossen, en landbouw en veeteelt. Klimaatadaptatie is een antwoord op zulke milieuveranderingen door te streven naar het terugdringen van de kwetsbaarheid van sociale en ecologische subsystemen door veranderingen in temperatuur, neerslag, zeeniveau, enzovoorts. Beleid gericht op klimaatadaptatie dient aandacht te schenken aan de gevolgen van klimaatveranderingen op zowel sociale als ecologische systemen, maar zeker ook aan de interactie tussen dergelijke systemen, hetgeen vraagt om een Sociaal-Ecologisch (SE) systeem perspectief.

In dit proefschrift beargumenteren we dat participatoire beleidsanalyse methoden cruciaal zijn voor besluitvorming met betrekking tot klimaatadaptatie in SE systemen. Dergelijke methoden gebruiken een diversiteit aan kennis, percepties, preferenties, en beslissingen van mensen die betrokken zijn bij het beheer en gebruik van, dan wel afhankelijk zijn van natuurlijke hulpbronnen, in de beleidsvoorbereiding. De participatoire beleidsanalyse benadering is ontstaan als antwoord op het falen van traditionele beleidsanalyse benaderingen die de nadruk leggen op formele (kwantitatieve) modellen, rationele planning en kosten-baten analyse bij het vinden van oplossingen voor allerlei complexe problemen. Wij beargumenteren dat de participatoire aanpak beleidsmakers helpt om de meest belangrijke aspecten van een SE systeem te adresseren in de beleidsvoorbereiding, te weten de complexiteit, de dynamiek en de onzekerheden in een dergelijk systeem.

De hoofddoelstelling van dit onderzoek is het ontwikkelen en demonstreren van participatoire beleidsanalyse methoden ter ondersteuning van het beleidsproces als sprake is van uitdagingen op milieugebied in SE systemen. Het onderzoek is gebaseerd op een case studie van een agrarische gemeenschap in Rafsanjan, Iran, die kampt met waterschaarste. Om de doelstelling te bereiken is een combinatie van participatoire methoden in een zekere volgorde ontworpen ten einde de kennis, percepties, preferenties en beslissingen van zowel boeren als beleidsmakers te kunnen integreren in de beleidsvoorbereiding met betrekking tot de water schaarste in Rafsanjan. In stap 1 is het Driver-Pressure-State-Impact-Response (DPSIR) kader gebruikt om het complexe SE system van water schaarste in de case studie te categoriseren en te structureren. In stap 2 is het DPSIR kader gecombineerd met een participatoire Fuzzy Cognitive Mapping methode (FCM, d.w.z. een methode voor kennis co-productie) waarbij op systematische wijze de kennis en percepties van boeren en beleidsmakers is verzameld over dat deel van het 
systeem waarvoor data schaars zijn, en waarbij deze informatie is gerepresenteerd in een semi-kwantitatief model. In stap 3 is de kwalitatieve kennis die gegenereerd is met de participatoire FCM gecombineerd met kwantitatieve data, via de ontwikkeling van een mixed-FCM die een representatie is van het complexe SE systeem van water schaarste. In stap 4 is het resultaat van de mixed-FCM gebruikt als invoer voor de ontwikkeling van een Agent-Based Model (ABM) om de beslissingen en acties van de boeren te modelleren en om het systeemgedrag op macro-niveau, dat het gevolg is van alle individuele gedragingen, te simuleren. Tenslotte zijn de gevolgen van opties voor overheidsbeleid gesimuleerd door de kennis, percepties en preferenties van stakeholders in de FCM modellen te integreren met de beslissingen en acties beschreven in het ABM.

Het resultaat van dit onderzoek is een beleidsondersteunende toolbox bestaande uit vier verschillende methoden, elk gericht op een andere probleem context of beleidsdoelstelling met betrekking tot klimaatadaptatie voor SE systemen. DPSIR is een probleem structurerend kader dat helpt bij het categoriseren en begrijpen van verschillende aspecten van het probleem voorafgaand aan de beleidsvoorbereiding. De Perceived-FCM is waardevol bij het voorspellen van de reacties van stakeholders op nieuwe beleidsmaatregelen. De Mixed-FCM kan worden gebruikt bij het simuleren en analyseren van de gevolgen van beleidsopties in SE systemen met meerdere factoren, waarbij zowel subjectieve als objectieve kennis relevant is voor een beter begrip van het systeemgedrag. Tenslotte is de FCM-based ABM waardevol bij het simuleren van de gevolgen van beleidsopties doordat het rekening houdt met feitelijk menselijk handelen en acties als ook met het meervoudige gedrag in SE systemen.

Deze studie vormt ook een innovatieve bijdrage aan 1) een geïntegreerd gebruik van kwalitatieve en kwantitatieve kennis in SE systeem modellering, 2) het modelleren van het gedrag van SE systemen op microniveau én macro-niveau, en 3) de combinatie van actor-based en factorbased benaderingen in SE systeem modellering. Deze studie introduceert beleidsondersteunende methoden die de belangrijkste aspecten van een complex SE systeem behandelen, namelijk causaliteit, feedback loops, sociaal-ruimtelijke heterogeniteit, en temporele dynamiek.

Tenslotte kan dit onderzoek waardevol zijn voor drie groepen geïnteresseerden: 1 . onderzoekers met interesse voor nieuwe methoden om SE systemen te modelleren, 2. professionals die participatoire beleidsanalyse methoden willen gebruiken, en 3. studenten die kunnen leren van de stapsgewijze aanpak die is ontwikkeld voor de verschillende aspecten in dit onderzoek voor hun toekomstige studies. 